UNIVERSIDADE DE SÃO PAULO

INSTITUTO DE ASTRONOMIA, GEOFÍSICA E CIÊNCIAS

ATMOSFÉRICAS

DEPARTAMENTO DE GEOFÍSICA

\begin{abstract}
ALLAN SEGOVIA SPADINI
Inversão da forma de onda completa de dados de sísmica de reflexão rasa
\end{abstract}




\section{Inversão da forma de onda completa de dados de sísmica de reflexão rasa}

Versão Corrigida. O original encontra-se disponível na Unidade.

Tese apresentada ao Instituto de Astronomia, Geofísica e Ciências Atmosféricas da Universidade de São Paulo para a obtenção do título de Doutor em Ciências.

Área de Concentração: Geofísica Aplicada

Orientadora: Prof ${ }^{a}$. Dra ${ }^{\mathrm{a}}$. Liliana Alcazar Diogo

Co-Orientador: Prof. Dr. Renato Luiz Prado 
Autorizo a reprodução e divulgação total ou parcial deste trabalho, por qualquer meio convencional ou eletrônico, para fins de estudo e pesquisa, desde que citada a fonte. 
Nome: SPADINI, Allan Segovia

Título: Inversão da forma de onda completa de dados de sísmica de reflexão rasa

Tese apresentada ao Instituto de Astronomia, Geofísica e Ciências Atmosféricas da Universidade de São Paulo para a obtenção do título de Doutor em Ciências.

Aprovado em: 15 de fevereiro de 2018

Banca Examinadora:

- Prof ${ }^{\mathrm{a}}$. Dr ${ }^{\mathrm{a}}$. Liliana Alcazar Diogo (orientadora) - (IAG/USP)

- Prof. Dr. Lúcio Tunes dos Santos (UNICAMP/Campinas-SP)

- Prof. Dr Emilson Pereira Leite - UNICAMP/Campinas-SP

- Dr. Otávio Coaracy Brasil Gandolfo - IPT/São Paulo

- Prof. Dr. Carlos Alberto Mendonça (IAG/USP) 


\section{Agradecimentos}

À minha orientadora Prof. Dra ${ }^{\mathrm{a}}$. Liliana Alcazar por todos estes anos apoio, paciência e dedicação que eu nunca vou esquecer.

Ao meu co-orientador Prof. Dr. Renato Luiz Prado, pelo incentivo, ajuda e por toda a paciência com as minhas dúvidas sobre Delft.

Thanks to Prof. Dr. Evert Slob for having received me in Delft and for the valuable suggestions during the time I spent under his guidance.

Ao Prof. Dr. Carlos Alberto Mendonça pelas avaliações e observações feitas em meus relatórios durante meu doutorado.

Ao Dr. Oleg Bokhonok por toda ajuda nos trabalhos de campo, sugestões, amizade e por saber o momento certo de se espantar as vacas.

Ao Prof. Dr. Marcelo Souza de Assumpção pelos questionamentos e sugestões valiosas.

Ao Prof. Dr. Prof. Jorge Luís Porsani coordenador do projeto da CNPq (406653/2013$5)$.

Às secretárias Teca e Virgínia do Departamento de Geofísica.

Aos técnicos Marcelo, Ernande e Zanon.

Ao amigo Julian David Realpe por ter ajudado no trabalho de campo e ter emprestado a churrasqueira.

À minha mãe e aos meus queridos amigos Luciana, Fábio, Márcia e Érica simplesmente por existirem.

À Sylvia Tamie por ter me apresentado a banda Móveis Coloniais de Acaju. Se algum dia ler este texto eu gostaria que soubesse que desejo que mesmo em tempos difíceis nunca desista dos seus sonhos.

Agradeço a CNPq pela bolsa de pesquisa processo (161498/2012-4).

De um modo geral agradeço a todos que ajudaram de alguma forma para a realização desta tese de doutorado. 
"I finally got permission. I told you I wanted to do something after the fight ended. A 100 year quest!!!"

Natsu Dragneel

(MASHIMA, 2017) 


\section{Resumo}

SPADINI, A. S. Inversão da forma de onda completa de dados de sísmica de reflexão rasa. 2018. n f. Tese (Doutorado) - Instituto de Astronomia, Geofísica e Ciências Atmosféricas, Universidade de São Paulo, 2018.

Este trabalho realizou um estudo sobre a aplicação de algoritmos de inversão da forma de onda completa (FWI) sobre dados de sísmica de reflexão em uma escala rasa ( 0 - $100 \mathrm{~m}$ de profundidade). A FWI foi estudada com o fim de melhorar as velocidades estimadas através do processamento de reflexão PP e PS convencional. Para um melhor entendimento da resposta obtida por este tipo de problema, a inversão foi avaliada sobre dados sintéticos por métodos de busca global e local. Na busca global foi utilizado o algoritmo de Evolução Diferencial que é uma variante de um algoritmo genético. O intuito da busca global foi avaliar a sensibilidade da função objetivo para cada parâmetro do modelo em diferentes janelas de afastamentos em relação à fonte. Na busca local foi utilizado um algoritmo de gradiente conjugado para a estimativa $2 \mathrm{D}$ dos parâmetros do meio. Dentre os principais resultados têm-se que a função objetivo é mais sensível aos parâmetros em janelas de afastamentos próximas da fonte. Em tais janelas, dominadas por ondas superficiais, a velocidade da onda S é facilmente estimada. Entretanto, mesmo em janelas mais afastadas a velocidade da onda S é o parâmetro do modelo que se destaca em relação aos demais. Já a busca por todos os parâmetros concomitantemente mostrou-se difícil e implicaria na necessidade de mais iterações do algoritmo de inversão. O método também foi aplicado em dados reais adquiridos no terreno do Instituto de Física da USP. A FWI foi aplicada nestes dados buscando apenas pelos valores de Vs, mantendo os valores de $\mathrm{Vp}$ e densidade fixos. A aplicação do algoritmo 2D nestes dados resultaram em valores de Vs coerentes com as velocidades observadas em um ensaio downhole na área. Concluindo, os resultados apresentados na tese mostram que a FWI é aplicável para a melhoria do modelo de velocidade da onda S obtido através do processamento de eventos de reflexão PP e PS.

Palavras-chave: FWI, processamento PP e PS, sísmica rasa. 


\section{Abstract}

SPADINI, A. S. Full waveform inversion of shallow seismic reflection data. 2018. $\mathrm{n} f$. Thesis (Phd.) - Instituto de Astronomia, Geofísica e Ciências Atmosféricas, Universidade de São Paulo, 2018.

This work carried out a study on the application of full waveform inversion algorithms (FWI) on reflection seismic data on a shallow scale (0 - $100 \mathrm{~m}$ depth). FWI has been studied in order to improve estimated velocities through conventional PP and PS reflection processing. For a better understanding of the response obtained by this type of problem the inversion was evaluated by global and local search methods. In the global search the algorithm employed was the Differential Evolution which is a variant of a genetic algorithm. The aim of the global search was to evaluate the sensitivity of the objective function for each parameter of the model in different windows of distance from the source. In the local search a conjugate gradient algorithm was used for a 2D estimate of the medium parameters. Among the main results is the fact that in a suitable window, for a reflection data acquisition the sensitivity is reduced in relation to a window with geophones closer to the source. However, even in more distant windows the velocity of the $\mathrm{S}$ wave is the parameter of the model that stands out in relation to the others. The concomitant search for all parameters at the same time is still difficult and implies the need for more iterations of the inversion algorithm. The method was also applied in a data acquired in the field of the Institute of Physics of USP. The results of the application of the 2D algorithm for this data showed modifications of the provided initial model for a velocity of the $\mathrm{S}$ wave coherent with the observed velocities in downhole and lithological informations from this area. In conclusion, the results found that FWI is applicable to improve the S-wave velocity model obtained by processing PP and PS reflection events.

Keywords: FWI, PP and PS processing, shallow seismic. 


\section{Lista de ilustrações}

Figura 1 - Distribuição das variáveis em um algoritmo de diferenças finitas de malha deslocada. . . . . . . . . . . . . . . . . . 18

Figura 2 - Representação do sólido linear padrão generalizado ou corpos de Maxwell. Aqui $k_{l}$ e $\eta_{l}(l=1, \ldots, \mathrm{L})$ representam os módulos elásticos e viscosidades Newtonianas, respectivamente. . . . . . . . . . . . . 22

Figura 3 - Função objetivo hipotética com pontos de mínimo local e globlal. . . . 24

Figura 4 - Fluxograma de uma iteração do algoritmo de FWI. . . . . . . . . . . . 25

Figura 5 - Efeito entre modificações no espaço dos modelos e no espaço dos dados. 29

Figura 6 - Fontes em uma aquisição sísmica. . . . . . . . . . . . . . . . . 35

Figura 7 - Conjunto de tiro de componente vertical criado a partir dos dados da Tabela 1). . . . . . . . . . . . . . . . . . 4 40

Figura 8 - Conjunto de tiro de componente radial criado a partir dos dados da Tabela 1). . . . . . . . . . . . . . . . . . . 44 40

Figura 9 - Análise de sensibilidade para a janela de 1 a 48 metros. Valor da função objetivo pela variação do parâmetro buscado. . . . . . . . . . . . . . . 41

Figura 10 - Análise de sensibilidade para a janela de 49 a 96 metros. Valor da função objetivo pela variação do parâmetro buscado. . . . . . . . . . . . . . . 42

Figura 11 - Função objetivo pelos parâmetros estimados. Pontos testados durante a aplicação do algoritmo de evolução diferencial. Janela de 1 a 48 metros. 44

Figura 12 - Função objetivo pelos parâmetros estimados. Pontos testados durante a aplicação do algoritmo de evolução diferencial. Janela de 49 a 96 metros. 45

Figura 13 - Modelos real e inicial para a velocidade da onda S. Velocidade constante nas camadas. . . . . . . . . . . . . . . . . . . 4 4

Figura 14 - Modelo de velocidade da onda S estimado no caso de um único tiro na posição de 5 metros. . . . . . . . . . . . . . . . . . . . . . . . . 4 4

Figura 15 - Resultado em detalhe extraído em diferentes posições do modelo de velocidades da onda $\mathrm{S}$ da Figura 14. . . . . . . . . . . . . . . . . 48

Figura 16 - Resultado para a estimativa da velocidade da onda S em uma aquisição com três pontos de tiro nas posições de 5,28 e 52 metros. . . . . . . . . 48

Figura 17 - Resultado em detalhe extraído em diferentes posições do modelo de velocidades da onda $\mathrm{S}$ da Figura 16. . . . . . . . . . . . . . . 49

Figura 18 - Modelos real, inicial e final para a inversão da velocidade da onda S em modelos com crescimento linear do parâmetro com a profundidade. . . 50

Figura 19 - Resultado em detalhe extraído em diferentes posições do modelo de velocidades da onda $\mathrm{S}$ da Figura 18. . . . . . . . . . . . . . . . 50 
Figura 20 - Resultado para a estimativa da velocidade da onda P no modelo de camadas em uma aquisição com três pontos de tiro nas posições de 5,28 e 52 metros. . . . . . . . . . . . . . . . . . . . .

Figura 21 - Resultado em detalhe extraído em diferentes posições do modelo de velocidades da onda P da Figura 20. . . . . . . . . . . . . . . . 51

Figura 22 - Resultado em detalhe extraído em diferentes posições do modelo de velocidades da onda $\mathrm{P}$ em um caso com uma diferença menor entre o modelo inicial e real. . . . . . . . . . . . . . . . . . . . . 52

Figura 23 - Resultado para a estimativa da velocidade da onda P no modelo com crescimento linear do parâmetro em uma aquisição com três pontos de tiro nas posições de 5,28 e 52 metros. . . . . . . . . . . . . . . . 52

Figura 24 - Resultado em detalhe extraído em diferentes posições do modelo de velocidades da onda P da Figura 23. . . . . . . . . . . . . . . . . . 53

Figura 25 - Resultado para a estimativa da densidade no modelo de camadas em uma aquisição com três pontos de tiro nas posições de 5,28 e 52 metros. 53

Figura 26 - Resultado em detalhe extraído em diferentes posições do modelo de densidades da Figura 25. . . . . . . . . . . . . . . . . 54

Figura 27 - Resultado para a estimativa da densidade no modelo com crescimento linear do parâmetro em uma aquisição com três pontos de tiro nas posições de 5,28 e 52 metros. . . . . . . . . . . . . . . . . . . 54

Figura 28 - Resultado em detalhe extraído em diferentes posições do modelo de densidades da Figura 27. . . . . . . . . . . . . . . . . . . . 55

Figura 29 - Resultado para a estimativa concomitante da velocidade das ondas P e $\mathrm{S}$ no modelo de camadas em uma aquisição com três pontos de tiro nas posições de 5,28 e 52 metros. . . . . . . . . . . . . . . . . 56

Figura 30 - Resultado em detalhe extraído em diferentes posições dos modelos da Figura 29. . . . . . . . . . . . . . . . . . . . 56

Figura 31 - Resultado para a estimativa concomitante da velocidade das ondas P e S no modelo com crescimento linear de parâmetros em uma aquisição com três pontos de tiro nas posições de 5,28 e 52 metros. . . . . . . . .

Figura 32 - Resultado em detalhe extraído em diferentes posições dos modelos da Figura 31. . . . . . . . . . . . . . . . .

Figura 33 - Resultado para a estimativa concomitante da velocidade das ondas P e $\mathrm{S}$ no modelo de camadas, onde Vp é estimada através de uma relação com Vs, em uma aquisição com três pontos de tiro nas posições de 5 , 28 e 52 metros. . . . . . . . . . . . . . . . . . . 58

Figura 34 - Resultado em detalhe extraído em diferentes posições dos modelos da Figura 33. . . . . . . . . . . . . . . . . . . . . . . 58 
Figura 35 - Resultado para a estimativa concomitante da velocidade das ondas P e $\mathrm{S}$ no modelo com crescimento linear de parâmetros e relação Vp e Vs em uma aquisição com três pontos de tiro nas posições de 5,28 e 52 metros.

Figura 36 - Resultado em detalhe extraído em diferentes posições dos modelos da Figura 35. . . . . . . . . . . . . . . . . .

Figura 37 - Resultado para a estimativa concomitante da velocidade das ondas P e $\mathrm{S}$ e da densidade no modelo de camadas em uma aquisição com três pontos de tiro nas posições de 5,28 e 52 metros. . . . . . . . . . . . . . 60

Figura 38 - Resultado em detalhe extraído em diferentes posições dos modelos da Figura 37. . . . . . . . . . . . . . . . . . . .

Figura 39 - Resultado para a estimativa concomitante da velocidade das ondas P e $\mathrm{S}$ e da densidade no modelo com crescimento linear de parâmetros em uma aquisição com três pontos de tiro nas posições de 5,28 e 52 metros. 62

Figura 40 - Resultado em detalhe extraído em diferentes posições dos modelos da Figura 39. . . . . . . . . . . . . . . . . . . . . 62

Figura 41 - Resultado para a estimativa concomitante da velocidade das ondas P e $\mathrm{S}$ e da densidade no modelo de camadas, onde $\mathrm{Vp}$ e a densidade são estimadas através de uma relação com Vs, em uma aquisição com três pontos de tiro nas posições de 5,28 e 52 metros. . . . . . . . . . . . . . 63

Figura 42 - Resultado em detalhe extraído em diferentes posições dos modelos da Figura 41. . . . . . . . . . . . . . . . . 6 64

Figura 43 - Resultado para a estimativa concomitante da velocidade das ondas P e $\mathrm{S}$ e da densidade no modelo com crescimento linear de parâmetros e relação de Vp e densidade com Vs em uma aquisição com três pontos de tiro nas posições de 5,28 e 52 metros.

Figura 44 - Resultado em detalhe extraído em diferentes posições dos modelos da Figura 43. . . . . . . . . . . . . . . . . .

Figura 45 - Pré-condicionamento do taper para um tiro na posição $\mathrm{x}=25 \mathrm{~m}$ na superfície livre. (a) modelo completo; (b) região do taper em detalhe.

Figura 46 - Resultado para a estimativa da velocidade da onda S no modelo de camadas em uma aquisição com três pontos de tiro nas posições de 5,28 e 52 metros. Cada resultado foi obtido para um valor diferente raio diferente para o afunilamento do gradiente na posição das fontes. . . . .

Figura 47 - Resultado para a estimativa da velocidade da onda S no modelo de onde os parâmetro crescem linearmente com a profundidade em uma aquisição com três pontos de tiro nas posições de 5,28 e 52 metros. Cada resultado foi obtido para um valor diferente raio diferente para o afunilamento do gradiente na posição das fontes. . . . . . . . . . . . . 
Figura 48 - Resultado para a estimativa da velocidade da onda S no modelo de camadas em uma aquisição com três pontos de tiro nas posições de 5,28 e 52 metros. Cada resultado com a Hessiana estimada por H1 com um valor de "nível d'água" diferente. . . . . . . . . . . . . . . . . . . . 69

Figura 49 - Resultado em detalhe extraído em diferentes posições dos modelos da Figura 48. . . . . . . . . . . . . . . . . . . . . 70

Figura 50 - Resultado para a estimativa da velocidade da onda S no modelo de crescimento linear dos parâmetros com a profundidade em uma aquisição com três pontos de tiro nas posições de 5,28 e 52 metros. Cada resultado com a Hessiana estimada por H1 com um valor de "nível d'água" diferente. 71

Figura 51 - Resultado em detalhe extraído em diferentes posições dos modelos da Figura 50. . . . . . . . . . . . . . . . . 72

Figura 52 - Resultado para a estimativa da velocidade da onda S no modelo de camadas em uma aquisição com três pontos de tiro nas posições de 5,28 e 52 metros. Cada resultado com a Hessiana estimada por H3 com um valor de "nível d'água" diferente. . . . . . . . . . . . . . . . . . . 73

Figura 53 - Resultado em detalhe extraído em diferentes posições dos modelos da Figura 52. . . . . . . . . . . . . . . . . . .

Figura 54 - Resultado para a estimativa da velocidade da onda $\mathrm{S}$ no modelo de crescimento linear dos parâmetros com a profundidade em uma aquisição com três pontos de tiro nas posições de 5,28 e 52 metros. Cada resultado com a Hessiana estimada por H3 com um valor de "nível d'água" diferente. 75

Figura 55 - Resultado em detalhe extraído em diferentes posições dos modelos da Figura 54. . . . . . . . . . . . . . . . . . . 76

Figura 56 - Resultados para a estimativa da velocidade da onda S para um modelo real de camada inclinada. . . . . . . . . . . . . . . . . . . . . 77

Figura 57 - Geofones de componente vertical (esquerda) e componente radial (direita). 78

Figura 58 - Onda P incidente e suas reflexões P e S em uma interface. . . . . . . . 79

Figura 59 - Possível fluxo de processamento para a reflexão PS . . . . . . . . . . . . 81

Figura 60 - Conjunto de tiro de componente radial com tiro realizado no centro do arranjo. . . . . . . . . . . . . . . . . . 82

Figura 61 - Distinção entre pontos CMP e CCP. . . . . . . . . . . . . . . . 83

Figura 62 - Caminhos percorridos por raios diretos e reversos de reflexões PP e PS em um meio com variação lateral de litologia. . . . . . . . . . . . . . . 84

Figura 63 - Tiro direto e tiro reverso de reflexão PS incidindo em um mesmo ponto. 86

Figura 64 - Distribuição dos pontos de reflexão: (a) refletor plano (coincide com a projeção do ponto CMP); (b) refletor com mergulho. . . . . . . . . . . 88

Figura 65 - Geometria do caminho do raio para uma reflexão PS em um refletor com mergulho. 
Figura 66 - Geometria do caminho do raio para uma reflexão PS em um refletor com mergulho com origem deslocada para o ponto médio. . . . . . . 89

Figura 67 - Mapa de localização da área estudada e perfil sísmico. . . . . . . . . . 91

Figura 68 - Correlação entre os perfis geológicos dos poços do sítio do Instituto de Física. . . . . . . . . . . . . . . . . . . . . . 92

Figura 69 - Perfil de velocidade para Vp e Vs para o poço P2. . . . . . . . . . . . 93

Figura 70 - Conjuntos de tiro adquiridos com geofones de componente vertical. . . 94

Figura 71 - Conjuntos de tiro adquiridos com geofones de componente radial. . . . 94

Figura 72 - Carta de empilhamento para a aquisição CMP da componente vertical. 95

Figura 73 - Modelo de velocidades Vp obtido a partir das primeiras chegadas observadas nos tiros de análise de ruído e nos conjuntos de tiro do levantamento de reflexão. . . . . . . . . . . . . . . . . . . . 996

Figura 74 - Ajuste das curvas de dispersão obtidas a partir dos conjuntos de tiro de análise de ruído. (a) Tiro direto; (b) Tiro reverso. . . . . . . . . . . . . 97

Figura 75 - Resultado da inversão das curvas de dispersão obtidas a partir conjuntos de tiro de análise de ruído. (a) Tiro direto; (b) Tiro reverso. . . . . . . 97

Figura 76 - Conjuntos de tiro de reflexão de componente vertical. (a) Dados brutos; (b) Após a aplicação do filtro passa-banda. . . . . . . . . . . . . . . . 98

Figura 77 - Conjuntos de tiro de reflexão de componente radial. (a) Dados brutos; (b) Após a aplicação do filtro passa-banda. . . . . . . . . . . . . . . . . 99

Figura 78 - Conjuntos de tiro de reflexão após a aplicação do filtro FK. (a) Componente Vertical; (b) Componente Radial. . . . . . . . . . . . . . . . . . 100

Figura 79 - Seção empilhada obtida a partir dos sismogramas de reflexão PP. . . . 101

Figura 80 - Seção empilhada ( para a onda PP) em profundidade. . . . . . . . . . . 101

Figura 81 - Seção empilhada obtida a partir dos sismogramas de reflexão PS. . . . 102

Figura 82 - Seção empilhada ( para a onda PS) em profundidade. . . . . . . . . . . 103

Figura 83 - Modelos iniciais para a aplicação de FWI no dado real. . . . . . . . . . 104

Figura 84 - Estimativa do valor do Q. (Cima) Espectro de amplitude para os traços 26 e 41 da componente vertical do conjunto de tiro 10; (Baixo) Valor estimado de Q. . . . . . . . . . . . . . . . . 105

Figura 85 - Resultado final para a estimativa da velocidade da onda S. . . . . . . . 105

Figura 86 - Comparação entre o modelo inicial e o modelo final na parte central da Figura 85. . . . . . . . . . . . . . . . 106 


\section{Lista de tabelas}

Tabela 1 - Propriedades físicas do modelo de camadas planas utilizado para a simulação do dado observado. . . . . . . . . . . . . . . . . . . . . . . 39

Tabela 2 - Perfil de velocidades extraído do poço P2. Extraído de Ullah, Prado e Lisa (2017) . . . . . . . . . . . . . . . . . . . 92 


\section{Sumário}

INTRODUÇÃO $\ldots \ldots \ldots \ldots \ldots \ldots \ldots$

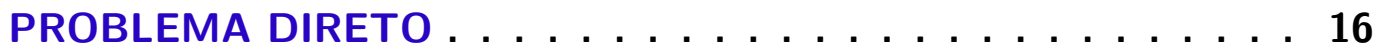

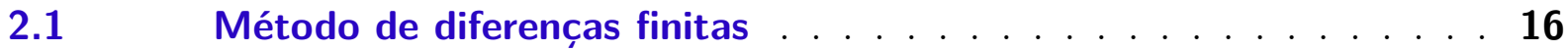

$2.1 .1 \quad$ Condições iniciais e de borda . . . . . . . . . . . . . . . . 18

2.1.2 Implementação das fontes . . . . . . . . . . . . . . 20

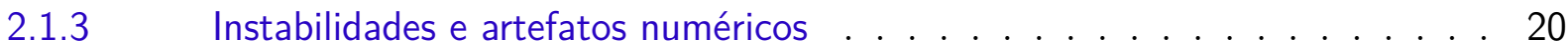

2.1.4 Extensão para o caso viscoelástico . . . . . . . . . . . . . . 21

3 PROBLEMA INVERSO . . . . . . . . . . . . . 24

3.1 Minimização local pelo método do gradiente conjugado não linear . 25

3.1.1 Estimativa inicial e atualização de parâmetros do modelo . . . . . . . . . 26

3.1.2 Gradiente da função objetivo . . . . . . . . . . . . . . . . . 27

3.1.3 Norma L2 e fonte adjunta correspondente . . . . . . . . . . . . . . 33

$3.1 .4 \quad$ Correção $3 \mathrm{D} / 2 \mathrm{D} \ldots \ldots \ldots \ldots . \ldots \ldots$

$3.1 .5 \quad$ Salto de ciclos . . . . . . . . . . . . . . . . . . . 34

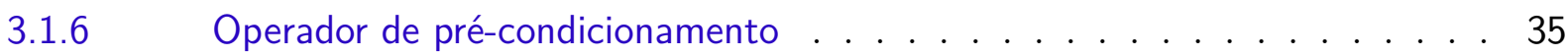

$3.2 \quad$ Busca global por evolução diferencial . . . . . . . . . . 36

3.3 Estimativa do fator de qualidade $\mathbf{Q} \ldots \ldots \ldots$

4 AVALIAÇÃO EM DADOS SINTÉTICOS . . . . . . . . . 39

4.1 Avaliação pela estratégia de otimização global de parâmetros . . . . 39

4.2 Testes com a estratégia local: algoritmo de inversão tomográfica . . 46

4.2.1 Análise da estimativa individual dos parâmetros . . . . . . . . . . . 46

4.2.2 Estimativa concomitante de parâmetros . . . . . . . . . . . . . . 55

4.2.3 Manipulação do gradiente . . . . . . . . . . . . . . 66

4.2.3.1 Afunilamento do gradiente . . . . . . . . . . . . . . . 66

4.2.3.2 Efeito do pré condicionamento do gradiente - Controle da aproximação da Hessiana 69

4.2.4 Modelo com camada inclinada . . . . . . . . . . . . . . . . . 76

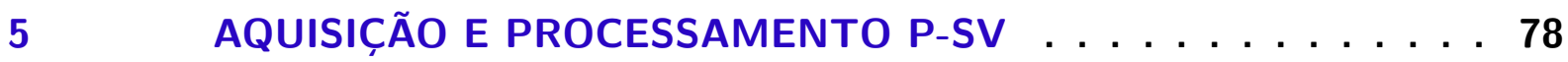

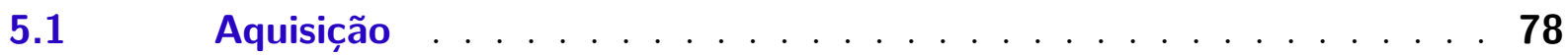

5.1 .1 Técnica Mini-Sosie . . . . . . . . . . . . . . . . . . . . . . . 79

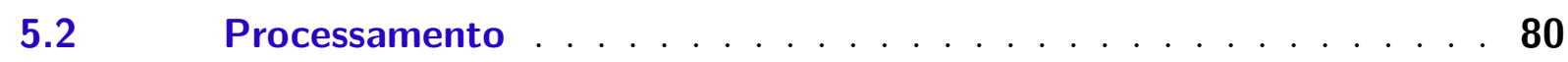

5.2.1 Organiação em conjuntos CCP . . . . . . . . . . . . . . . 82

$5.2 .2 \quad$ Análise de velocidades . . . . . . . . . . . . . . . . 85 
5.2.3 Correção de sobretempo normal (NMO) . . . . . . . . . . . . 87

5.2.4 Migração pós-empilhamento . . . . . . . . . . . . . 89

6 DADOS DO TERRENO DO INSTITUTO DE FÍSICA DA USP . . 91

6.1 Processamento de refração . . . . . . . . . . . . 96

6.2 Processamento de ondas superficiais . . . . . . . . . . . 96

6.3 Processamento de reflexão . . . . . . . . . . . . . . 97

6.3.1 Processamento de reflexões PP . . . . . . . . . . . . . . . 101

6.3.2 Processamento de reflexões PS . . . . . . . . . . . . . . . . 102

$6.3 .3 \quad$ Aplicação da FWI . . . . . . . . . . . . . . . . 103

7 DISCUSSÃO E CONCLUSÃO . . . . . . . . . . . . 107

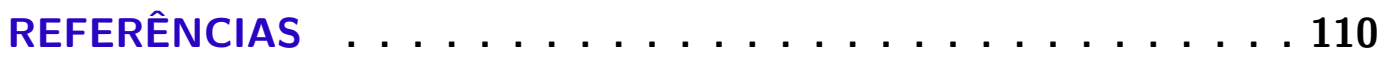




\section{Introdução}

A inversão da forma de onda completa (FWI), do inglês Full Waveform Inversion, é um tipo de inversão tomográfica que utiliza todos os eventos presentes em um sismograma. Este método é utilizado para estimar modelos de velocidade de alta resolução. Os modelos são obtidos através da minimização da diferença entre os sismogramas observados e os gerados a partir da solução numérica da equação de onda. A FWI pode ser uma opção para a interpretação de dados sísmicos multicomponente na escala da investigação rasa (até em torno de $100 \mathrm{~m}$ de profundidade), além de apresentar algumas vantagens comparativamente ao método de reflexão sísmica convencionalmente empregado, tais como i) interpretação das reflexões, independente da escolha de uma janela ótima de afastamentos; utilização das ondas convertidas PS, sem a necessidade de organizar traços sísmicos em sismogramas CCP (common conversion point); iii) estimativa das camadas muito rasas, nos primeiros metros em subsuperfície; e iv) identificação de regiões anômalas.

Para o processamento convencional de dados de reflexão sísmica rasa é importante identificar a janela ótima de afastamentos, entre fonte e receptores, de forma a evitar a interferência de eventos coerentes como o ground-roll, onda área e refrações. Este procedimento leva, usualmente ao emprego de afastamentos longos em relação à profundidade dos refletores. Entretanto, com um valor alto para a razão afastamento/profundidade a estimativa de velocidades por equações de tempo de percurso como a hiperbólica se torna inválida. Este problema afeta as reflexões PP, registradas normalmente em geofones de componente vertical e principalmente as reflexões PS, com maior parte da energia registrada em geofones de componente horizontal orientados na direção da linha de aquisição (componente radial).

Outro problema do método de reflexão é que normalmente é difícil visualizar eventos de reflexões em camadas muito rasas. Mesmo que interfaces possam ser interpretadas a partir dos eventos de refração, apenas o modelo de velocidade da onda $\mathrm{P}\left(V_{P}\right)$ em profundidade é obtido, usualmente. A velocidade da onda $\mathrm{S}$ tem de ser estimada de outra forma, como através da inversão das curvas de dispersão das ondas superficiais, por exemplo.

Na FWI é adotada a solução da equação elástica para a obtenção da forma de onda completa. Assim, toda a informação presente nos sismogramas pode ser utilizada. Desta forma, resolve-se os parâmetros de forma conjunta reduzindo ambiguidades e refinando modelos fornecidos por diferentes técnicas.

A FWI foi proposta por Tarantola (1984) utilizando uma aproximação acústica. Esta metodologia foi posteriormente estendida para o caso elástico em Tarantola (1986) e em Mora (1987) e para o caso viscoelástico por Tarantola (1988). Existem diversas aplicações da FWI para escala de petróleo e para a sismologia. Virieux e Operto (2009) 
e Fichtner (2011) trazem boas revisões sobre o tema. Entretanto, o interesse pela escala rasa surgiu apenas recentemente. Em Romdhane et al. (2011) é apresentado um algoritmo de FWI 2D no domínio da frequência para o estudo de um caso sintético com topografia complexa. Nos trabalhos de Tran e Hiltunen (2012a) e Tran e Hiltunen (2012b) foram utilizados algoritmos de busca global aplicados em modelos simplificados, com parâmetros constantes nas camadas. Em Tran e McVay (2012), Tran et al. (2013a) e Mcvay e Tran (2013) foi buscado um modelo de velocidades 2D de alta resolução através de um algoritmo Gauss-Newton no domínio do tempo. Seguindo a linha de estimativa 2D a partir de dados sísmicos na escala rasa, citam-se os trabalhos de Schafer et al. (2013) onde foi aplicado um algoritmo de gradiente conjugado na tentativa de extrair informação da forma de onda completa em sismogramas dominados por ondas superficiais em uma linha transversal a uma falha. O trabalho de Bretaudeau et al. (2013), utilizou dados sintéticos e dados provenientes de modelo físicos em escala reduzida para uma aplicação de FWI no domínio da frequência em dados também dominados por ondas superficiais. Em Groos et al. (2014a) foi estudado o efeito da atenuação anelástica na FWI de ondas Rayleigh. Amrouche e Yamanaka (2015) também desenvolveram uma FWI no domínio do tempo para a inversão de ondas superficiais. Tran e Luke (2017) utilizaram um método de Gauss-Newton para a caracterização de um aluvião de um deserto. Nestes exemplos de inversão 2D é realizado refinamento de um modelo inicial através de um algoritmo de otimização local. Nestas aplicações voltadas para engenharia e geotecnia, ocorre uma busca por modelos de velocidade da onda $\mathrm{S}$ em situações bastante rasas em torno de 15 metros, trabalhando com intervalos de frequência e afastamentos restritos.

Um caminho diferente em relação aos trabalhos citados seria aplicar a FWI sobre dados de sísmica de reflexão rasa. Os trabalhos de Romdhane et al. (2011) e Feng, Ren e Liu (2017) são exemplos sintéticos mais próximos desta possibilidade, sendo que o último é um exemplo de inversão conjunta com o método GPR. E em Yuan, Simons e Bozdă̆ (2015) foi aplicada a FWI sobre ondas superficiais e de corpo, isto foi realizado aplicando diferentes funções objetivo para cada tipo de evento. A inversão de dados adquiridos para o imageamento na sísmica de reflexão pode tirar proveito dos eventos de refração e ondas superficiais, independente da janela de afastamentos utilizada para a aquisição dos dados. Além disso, na busca da estimativa de modelos mais próximos à superfície, a má escolha dos parâmetros de pré-condicionamento do gradiente, nos algoritmos de busca, ou altas amplitudes próximas a posição das fontes podem ocasionar efeitos indesejáveis na resposta final, os quais merecem ser avaliados. Assim, este tipo de estudo pode ajudar a otimizar a aquisição e o procedimento de inversão além de permitir a avaliação do método sob uma perspectiva diferente.

Do exposto acima, nota-se que resta uma lacuna em relação a aplicação da técnica de FWI sobre dados de reflexão rasa. Com isto, na tese foi desenvolvido um estudo na escala de sísmica rasa utilizando todos os eventos presentes em sismogramas de reflexão 
de componentes vertical e radial. Foram avaliadas a sensibilidade na estimativa de cada parâmetro do modelo e a influência das profundidades e afastamentos relativos a um levantamento de sísmica de reflexão rasa na solução do problema.

Os capítulos 2, 3 e 5 compreendem a parte teórica. No capítulo 2 é realizada uma revisão do método de diferenças finitas utilizado na modelagem da forma de onda completa. O capítulo 3 compreende a teoria do método de gradiente conjugado utilizado para a aplicação da FWI e a teoria do método de evolução diferencial que foi utilizado como forma de inversão global e para melhor entendimento do problema. Além disso, foram descritos aspectos importantes da aplicação de FWI. No capítulo 5 são discutidos conceitos da aquisição e processamento de dados de reflexões PP e PS.

No capítulo 4 é apresentado um estudo sobre dados sintéticos. É apresentado um estudo da sensibilidade de cada parâmetro do modelo, assim como diversas avaliações dos parâmetros adotados na aplicação do método de gradiente conjugado para realizar a FWI.

No capítulo 6 é apresentado um estudo de caso de um dado de sísmica de reflexão multicomponente adquirido no campus Cidade Universitária da Universidade de São Paulo, em local sobre sedimentos da Bacia de São Paulo.

No capítulo 7 são apresentadas as conclusões. 


\section{Problema Direto}

O problema direto é a simulação da resposta física do meio à propagação de ondas sísmicas. A solução da equação de onda com este intuito é essencial no método de FWI.

Na aplicação da FWI a solução da equação de onda é obtida por métodos diretos. Seguindo a definição de Carcione, Herman e Kroode (2002) os métodos diretos, também chamados de métodos de malha ou métodos de equação de onda completa, são utilizados discretizando o modelo geológico em um número finito de pontos. Exemplos de métodos diretos são os métodos de elementos finitos, métodos pseudo espectrais e o método de diferenças finitas. Neste trabalho será utilizado o método de diferenças finitas no domínio do tempo que já foi aplicado e validado em trabalhos como os de Tran e Hiltunen (2012a) e Tran e Hiltunen (2012b) e Groos et al. (2017). Neste trabalho tanto para a parte da modelagem direta quanto da modelagem inversa foi utilizado o código de nome DENISE Köhn et al. (2010) atualmente IFOS2D (GROOS et al., 2017).

\subsection{Método de diferenças finitas}

Para a construção de sismogramas sintéticos foi adotado o método de diferenças finitas de malha deslocada (VIRIEUX, 1986), (LEVANDER, 1988). Outros esquemas de diferenças finitas são explicados no trabalho de Moczo (1998).

Assumindo um espaço 3D e um sólido homogêneo e isotrópico a equação de movimento pode ser escrita como:

$$
\rho \frac{\partial^{2} \vec{u}}{\partial t^{2}}=\frac{\partial \sigma_{i j}}{\partial x_{j}}+\vec{f}
$$

onde $\rho$ é a densidade em $\mathrm{kg} / \mathrm{m}^{3}$, t é o tempo, $\vec{u}$ é o vetor de deslocamento da partícula, $\sigma_{i} j$ são as tensões em $N / m^{2}$ e $\vec{f}$ a densidade de força de volume em $N / m^{3}$.

Com uma formulação tensão-velocidade da partícula para a equação de movimento um meio $2 \mathrm{D}(x, z)$ todas as derivadas com respeito a $y$ desaparecem e a propagação de ondas $\mathrm{P}-\mathrm{Sv}$ pode ser escrita por:

$$
\begin{aligned}
& \rho \frac{\partial^{2} \vec{u}_{x}}{\partial t^{2}}=\frac{\partial \sigma_{x x}}{\partial x}+\frac{\partial \sigma_{x z}}{\partial z}+\vec{f}_{x} \\
& \rho \frac{\partial^{2} \vec{u}_{z}}{\partial t^{2}}=\frac{\partial \sigma_{z x}}{\partial x}+\frac{\partial \sigma_{z z}}{\partial z}+\vec{f}_{z} .
\end{aligned}
$$

As leis constitutivas de um meio elástico isotrópico (relação de tensão-deformação) são dadas pela lei de Hooke e podem ser escritas como: 


$$
\begin{aligned}
\sigma_{x x} & =(\lambda+2 \mu) \frac{\partial u_{x}}{\partial x}+\lambda \frac{\partial u_{z}}{\partial z}, \\
\sigma_{z x} & =\mu\left(\frac{\partial u_{x}}{\partial z}+\frac{\partial u_{z}}{\partial x}\right), \\
\sigma_{z z} & =(\lambda+2 \mu) \frac{\partial u_{z}}{\partial z}+\lambda \frac{\partial u_{x}}{\partial x},
\end{aligned}
$$

onde $\lambda$ e $\mu$ são os parâmetros de Lamé.

Os termos $\partial u_{x} / \partial t$ e $\partial u_{z} / \partial t$ podem ser expressados também por $v_{x}$ e $v_{z}$, velocidades das partículas. A equação 2.2 pode ser diferenciada em relação ao tempo para que seja obtida a relação entre a derivada em tempo das tensões e velocidades da partícula. Com isto, as derivadas parciais podem ser aproximadas por operadores de diferenças finitas.

Um operador de avanço de segunda ordem $D_{k}^{+}$pode ser utilizado para aproximar a derivada de uma função genérica $a$ com espaçamento de malha $\Delta x$ da seguinte forma:

$$
\left.\frac{\partial a(i \Delta x)}{\partial x}\right|_{(i+1 / 2) \Delta x} \approx D_{x}^{+}[a(i)]=\frac{1}{\Delta x}[a(i+1)-a(i)] .
$$

E um operador de atraso como

$$
\left.\frac{\partial a(i \Delta x)}{\partial x}\right|_{(i-1 / 2) \Delta x} \approx D_{x}^{-}[a(i)]=\frac{1}{\Delta x}[a(i)-a(i-1)] .
$$

Fazendo uso deste tipo de operador, utilizando $i$ e $j$ como índices das posições no plano cartesiano $x$ e $z$ e considerando que os símbolos $+\mathrm{e}-$ indicam posições deslocadas em metade do tamanho de uma célula, pontos intermediários da malha. O campo de velocidades baseado nas equações 2.2 e 2.3 pode ser atualizado como segue no sistema:

$$
\begin{aligned}
& v_{x}^{n+1}\left(i^{+}, j\right)=v_{x}^{n+1}\left(i^{+}, j\right)+\frac{\Delta t}{\rho\left(i^{+}, j\right)}\left(D_{x}^{+}\left[\sigma_{x x}^{n^{+}}(i, j)\right]+D_{z}^{+}\left[\sigma_{x z}^{n+}\left(i^{+}, j^{+}\right)\right]+f_{x}^{n+}\right) \\
& v_{z}^{n+1}\left(i, j^{+}\right)=v_{z}^{n}\left(i, j^{+}\right)+\frac{\Delta t}{\rho\left(i, j^{+}\right)}\left(D_{x}^{-}\left[\sigma_{x z}^{n+}\left(i^{+}, j^{+}\right)\right]+D_{z}^{+}\left[\sigma_{z z}^{n+}(i, j)\right]+f_{z}^{n+}\right) \\
& \sigma_{x x}^{n+}(i, j)=\sigma_{x x}^{n-}(i, j)+\Delta t\left((\lambda(i, j)+2 \mu(i, j)) D_{x}^{-}\left[v_{x}^{n}\left(i^{+}, j\right)\right]+\lambda(i, j) D_{z}^{-}\left[v_{z}^{n}\left(i, j^{+}\right)\right]\right) \\
& \sigma_{z z}^{n+}(i, j)=\sigma_{z z}^{n-}(i, j)+\Delta t\left(\lambda(i, j) D_{x}^{-}\left[v_{x}^{n}\left(i^{+}, j\right)\right]+(\lambda(i, j)+2 \mu(i, j)) D_{z}^{-}\left[v_{z}^{n}\left(i, j^{+}\right)\right]\right) \\
& \sigma_{x z}^{n+}\left(i^{+}, j^{+}\right)=\sigma_{x z}^{n-}\left(i^{+}, j^{+}\right)+\mu\left(i^{+}, j^{+}\right) \Delta t\left(D_{x}^{+}\left[v_{z}^{n}\left(i, j^{+}\right)\right]+D_{z}^{-}\left[v_{x}^{n}\left(i^{+}, j\right)\right]\right)
\end{aligned}
$$

onde $\Delta t$ é o intervalo de amostragem no tempo e $n$ é o índice que marca o passo no tempo. O parâmetro do modelo $\rho$ em pontos intermediários da malha é calculado pela média de dois valores vizinhos de densidade localizados em pontos da malha: 


$$
\begin{aligned}
& \overline{\rho_{x}}=\rho\left(i^{+}, j\right)=\frac{1}{2}[\rho(i, j)+\rho(i+1, j)] \\
& \overline{\rho_{z}}=\rho\left(i, j^{+}\right)=\frac{1}{2}[\rho(i, j)+\rho(i, j+1)]
\end{aligned}
$$

O tensor de deformação é deslocado por metade do tamanho de uma célula tanto na direção $x$ quanto na direção $z$ de forma que $m u$ é obtido pela média harmônica através dos seus quatro pontos vizinhos.

$$
\langle\mu\rangle=\mu\left(i^{+}, j^{+}\right)=4\left[\frac{1}{\mu(i, j)}+\frac{1}{\mu(i+1, j)}+\frac{1}{\mu(i, j+1)}+\frac{1}{\mu(i+1, j+1)}\right]^{-1}
$$

A distribuição das variáveis em uma malha deslocada com precisão de segunda ordem no tempo e quarta ordem no espaço pode ser visualizada na Figura 1

Figura 1 - Distribuição das variáveis em um algoritmo de diferenças finitas de malha deslocada.



Fonte: Köhn (2011).

\subsubsection{Condições iniciais e de borda}

As condições iniciais para um problema elástico são

$$
\begin{aligned}
& \vec{u}=0 \\
& \frac{\partial \vec{u}}{\partial t}=0
\end{aligned}
$$

para todos os pontos do modelo no instante $t=0$.

Na parte superior do modelo deve ser implementada a condição de superfície livre 
e nas outras três bordas devem ser tomadas medidas para a atenuação das reflexões que ocorrem nos limites do modelo.

A condição de superfície livre horizontal é implementada considerando que na interface entre o meio elástico e o ar todas as tensões na direção normal somem de forma que

$$
\sigma_{x z}=\sigma_{z z}=0
$$

No código DENISE é utilizada a técnica de espelhamento ou imagem proposta por Levander (1988). Esta forma leva a soluções estáveis e precisas para uma superfície plana. Se a superfície plana estiver localizada em um ponto $i=h$ da malha, o estresse neste ponto é levado para zero e os estresses abaixo deste ponto são espelhados com sinal reverso de forma que

$$
\begin{aligned}
& \sigma_{z z}(h, j)=0 \\
& \sigma_{z z}(h-1, j)=-\sigma_{z z}(h, j) \\
& \sigma_{x z}\left(h-\frac{1}{2}, j+\frac{1}{2}\right)=-\sigma_{x z}\left(h+\frac{1}{2}, j+\frac{1}{2}\right) \\
& \sigma_{x z}\left(h-\frac{3}{2}, j+\frac{1}{2}\right)=-\sigma_{x z}\left(h+\frac{3}{2}, j+\frac{1}{2}\right)
\end{aligned}
$$

Com isto, apenas a componente $\sigma_{x x}=(\lambda+2 \mu) v_{x x}+\lambda v_{z z}$ deve ser atualizada utilizando apenas velocidades da partícula. Segundo Levander (1988) as derivadas verticais sobre a superfície livre levam a instabilidades. A derivada vertical da velocidade da partícula em $z\left(v_{z z}\right)$ pode ser substituída usando a condição de borda na superfície livre:

$$
\begin{aligned}
& \sigma_{z z}=d t(\lambda+2 \mu) v_{z z}+d t \lambda v_{x x}=0 \\
& v_{z z}=-\frac{\lambda}{\lambda+2 \mu} v_{x x} .
\end{aligned}
$$

Portanto a tensão $\sigma_{x x}$ pode ser escrita como

$$
\sigma_{x x}=\frac{4 d t\left(\lambda \mu+\mu^{2}\right.}{\lambda+2 \mu} v_{x x}
$$

A condição de superfície livre torna possível a presença de ondas superficiais.

Para as demais bordas do modelo é necessário aplicar condições de absorção. O método de camadas perfeitamente compatíveis do inglês Perfectly Matched Layers (PMLs) é implementado através de um estiramento das coordenadas da equação de onda no domínio da frequência.

Em Groos et al. (2017) é explicado que este estiramento das coordenadas cria soluções de ondas planas que decaem exponencialmente na borda PML adotada no modelo e só ocorrem reflexões nas camadas se a equação de onda exata for resolvida. Na forma discreta o campo de onda deve ser atenuado por uma função do tipo

$$
c=-V_{p m l} \frac{\log (\alpha)}{L}
$$


onde $V_{p m l}$ é a velocidade típica da onda $\mathrm{P}$ no meio na camada de absorção, $\alpha=1 \times 10^{-4}$ e L é a espessura da camada de absorção.

\subsubsection{Implementação das fontes}

As fontes podem ser inseridas na equação de onda através da adição de densidades de forças de volume $\vec{f}$ como na equação 2.1. Aqui será discutida a implementação de forças diretas verticais por estas se aproximarem mais das fontes impulsivas utilizadas em sísmica rasa.

Considerando uma fonte de força linear vertical ao longo do eixo y, a densidade de força é dada por

$$
\vec{f}(\vec{x}, t)=F_{0}^{\prime} s(t) \delta(x) \delta(z) \hat{e_{z}}
$$

onde $F_{0}^{\prime}$ é a densidade de linha de força em N/m, s(t) é a função adimensional temporal da fonte, $\delta(x)$ e $\operatorname{delta}(z)$ são deltas de Dirac com $1 / \mathrm{m}$ e $\hat{e_{z}}$ é um vetor unitário na direção z.

A forma discreta da densidade de força em $(\mathrm{i}, \mathrm{j})$ e passo em tempo n pode ser escrita assumindo que $\delta(x) \approx 1 / \Delta h$ em um algoritmo de diferenças finitas com espaçamento $\Delta h$ da malha. O espaçamento da malha é igual nas duas direções. Desta forma,

$$
f_{z}^{n}(i, j)=\frac{F_{0}^{\prime}}{\Delta h^{2}} s^{n}
$$

Esta pode ser aplicada através da atualização da velocidade da partícula (equação 2.6 por:

$$
v_{z}^{n+1}\left(i, j^{+}\right)=v_{z}^{n}\left(i, j^{+}\right)+\frac{\Delta t}{\rho\left(i, j^{+}\right)}\left(D_{x}^{-}\left[\sigma_{x z}^{n+}\left(i^{+}, j^{+}\right)\right]+D_{z}^{+}\left[\sigma_{z z}^{n+}(i, j)\right]+\frac{F_{0}^{\prime}}{\Delta h^{2}} s^{n+}\right) .
$$

\subsubsection{Instabilidades e artefatos numéricos}

A utilização de espaçamentos inadequados tanto no espaço quanto no intervalo de tempo podem levar a instabilidades e artefatos numéricos. O efeito de dispersão de malha é observado quando o espaçamento dos pontos da malha é muito grande. Neste caso, o campo de onda modelado aparenta ser dispersivo. Para evitar esse efeito, o espaçamento dh deve respeitar a regra:

$$
d h<=\frac{V_{\min }}{b_{1}} f_{\max }
$$

onde $V_{\min }$ é a menor velocidade no modelo, $f_{\max }$ é a máxima frequência da fonte e $b_{1}$ é um parâmetro que depende da precisão do operador de diferenças finitas.

Outro fator importante é a instabilidade de Courant. Essa instabilidade é relacionada 
ao intervalo de tempo. O intervalo dt deve ser menor que o tempo que a onda leva para viajar em dois pontos adjacentes considerando um espaçamento dh. Assim:

$$
d t<=\frac{d h}{b_{2} \sqrt{2} V_{\max }}
$$

onde $V_{\max }$ é a velocidade máxima no modelo e $b_{2}$ é termo um dependente da ordem do operador de diferenças finitas.

\subsubsection{Extensão para o caso viscoelástico}

Segundo Igel (2016) reologias não isotrópicas são importantes para aproximações realísticas e são utilizadas nos códigos atuais. No caso dos materiais sólidos as reologias descrevem como as tensões e estresses se relacionam. No código DENISE é possível simular a viscoelasticidade do meio. Este tipo de efeito é observado na sísmica, devido a microdanos causados pela passagem das ondas ou conversão em calor induzida por fricção.

Para a modelagem viscoelástica pode ser utilizado um modelo chamado de "sólido linear padrão generalizado"(GSLS). Uma proposta baseada neste tipo de modelo foi apresentada em Emmerich e Korn (1987) para a propagação de ondas escalares. Em Bohlen (2002) foi desenvolvido um código baseado no modelo GSLS para a inclusão de efeitos viscoelásticos em um algoritmo de modelagem. Moczo et al. (2007) fornecem uma visão detalhada sobre a incorporação de atenuação em esquemas de diferenças finitas.

A Figura 2 mostra o modelo GSLS em detalhe. Neste modelo L corpos de Maxwell (cordas $k_{l}$ e amortecedores $\eta_{l}$ em série $\mathrm{l}=1, \ldots, \mathrm{L}$ ) são conectados em paralelo com a corda $k_{0}$. Onde $k_{l}$ e $\eta_{l}$ representam módulos elásticos e viscosidades Newtonianas, respectivamente. Desta forma, o módulo complexo M de um modelo GSLS pode ser descrito no domínio da frequência como

$$
M(\omega)=k_{0}\left[1-L+\sum_{l=1}^{L} \frac{1+i \omega \tau_{\epsilon l}}{1+i \omega \tau_{\sigma l}}\right]
$$

onde $\omega$ é a frequência angular, $\tau_{\sigma l}$ é o tempo de relaxação das tensões e $\tau_{\epsilon l}$ é o tempo de relaxação das deformações. Os dois últimos estão conectados com os respectivos $\left(k_{l}\right.$ e $\left.\eta_{l}\right)$ do modelo GSLS por

$$
\tau_{\sigma l}=\frac{\eta_{l}}{k_{l}}
$$

$\mathrm{e}$

$$
\tau_{\epsilon l}=\frac{\eta_{l}}{k_{0}}+\frac{\eta_{l}}{k_{l}}
$$


O fator de qualidade Q é utilizado para descrever as propriedades de atenuação das rochas através de

$$
Q=\frac{\Re[M(\omega)]}{\Im[M(\omega)]}
$$

Figura 2 - Representação do sólido linear padrão generalizado ou corpos de Maxwell. Aqui $k_{l}$ e $\eta_{l}(\mathrm{l}=1, \ldots, \mathrm{L})$ representam os módulos elásticos e viscosidades Newtonianas, respectivamente.

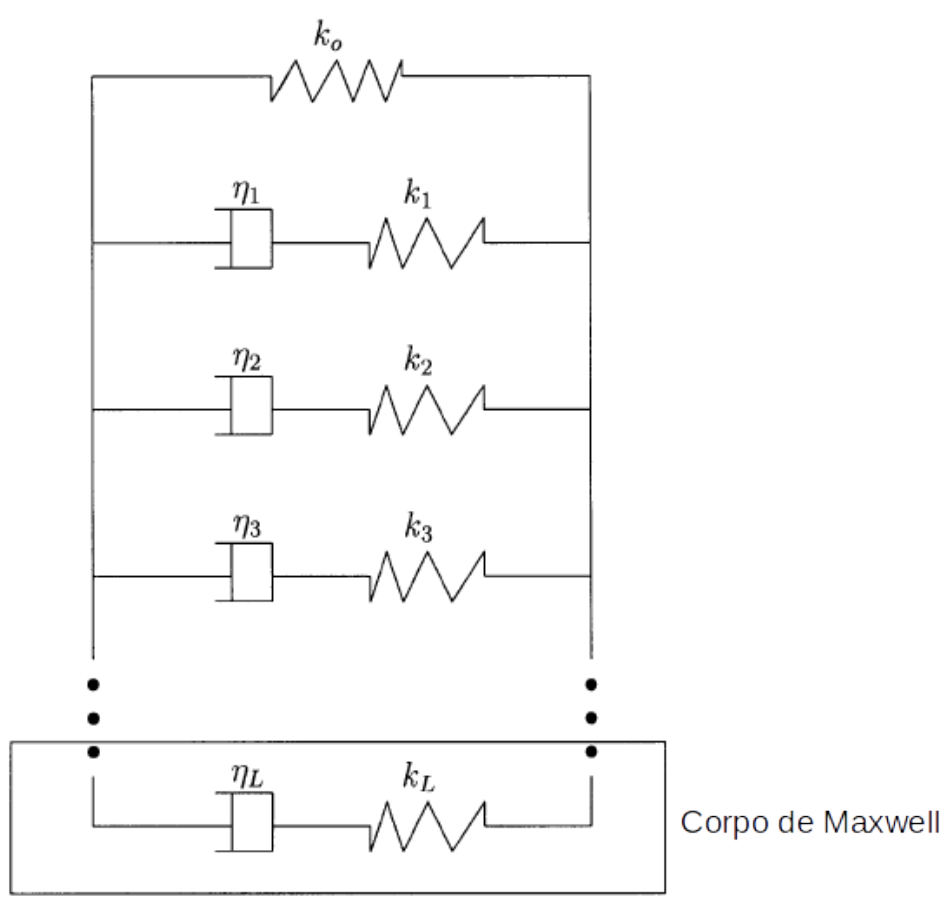

Fonte: Bohlen (2002).

Utilizando as equações (2.20) e (2.23) o fator de qualidade Q para um modelo GSLS pode ser escrito como

$$
Q\left(\omega, \tau_{\sigma l}, \tau\right)=\frac{1+\sum_{l=1}^{L} \frac{\omega^{2} \tau_{\sigma l}^{2}}{1+\omega^{2} \tau_{\sigma l}^{2}} \tau}{\sum_{l=1}^{L} \frac{\omega \tau_{\sigma l}}{1+\omega^{2} \tau_{\sigma l}^{2}} \tau}
$$

onde

$$
\tau=\frac{\tau_{\epsilon l}}{\tau_{\sigma l}}-1
$$

As variáveis $\tau_{\sigma l}$ e $\tau$ são determinadas através da minimização da seguinte função por mínimos quadrados

$$
J\left(\tau_{\sigma l}, \tau\right):=\int_{\omega_{1}}^{\omega_{2}}\left[Q^{-1}\left(\omega, \tau_{\sigma l}, \tau\right)-\tilde{Q}^{-1}\right]^{2} d \omega
$$


onde $\tilde{Q}$ é a constante $Q$ desejada.

Esta otimização deve ser realizada para as ondas P e S. Este processo retorna valores $\tau$ para as duas ondas que serão denotados por $\tau^{P}$ e $\tau^{S}$. Os mesmos valores de relaxação $\tau_{\sigma l}$ podem ser usados para as duas ondas. 


\section{Problema Inverso}

No problema inverso queremos garantir o ajuste aos dados observados. O modelo $\mathrm{m}$ de valores teóricos permite a construção dos sismogramas modelados $u_{\text {mod }}$, como descrito no capítulo anterior. Desta forma queremos que o resíduo $\delta u=u_{\text {mod }}-u_{o b s}$ entre os sismogramas modelados e os observados seja mínimo. Para isto procuramos o mínio de um funcional E, que como ilustrado na Figura 3 pode conter mínimos locais e globais. Sendo que métodos de minimização local, como o do gradiente conjugado, precisam de modelos iniciais que levam a pontos próximos o suficiente do ponto de mínimo global. Uma opção é a utilização conjunta de algoritmos de busca global e local para a obtenção de um valor ótimo.

Aqui o problema foi avaliado utilizando tanto métodos de Evolução Diferencial de Storn e Price (1997) que pode ser entendido como uma variante de Algoritmos Genéticos. A aplicação do algoritmo de busca global teve o objetivo de buscar versões paramétricas simples do meio visando um melhor entendimento do problema e construção de uma aproximação inicial. Já para a busca local através de uma abordagem tomográfica foi utilizado um algoritmo de gradiente conjugado desenvolvido por Köhn (2011). Neste último foram realizadas modificações para o tipo de aquisição estudada tentando garantir a convergência para a resposta desejada.

Figura 3 - Função objetivo hipotética com pontos de mínimo local e globlal.

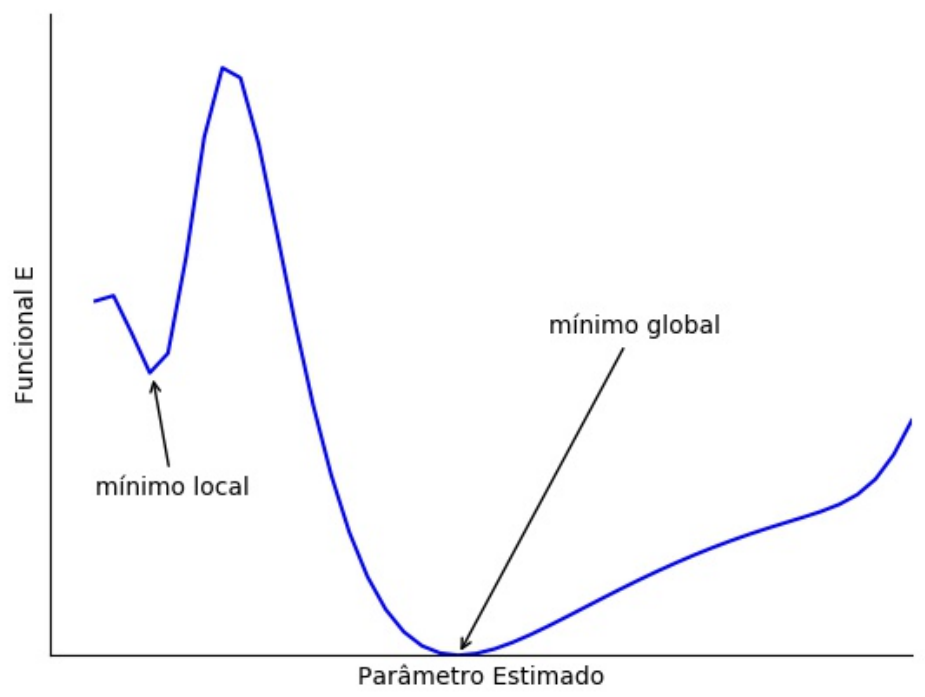




\subsection{Minimização local pelo método do gradiente conjugado não linear}

Uma visão geral do algoritmo de FWI com o gradiente conjugado pode ser visto na Figura 4.

Figura 4 - Fluxograma de uma iteração do algoritmo de FWI.

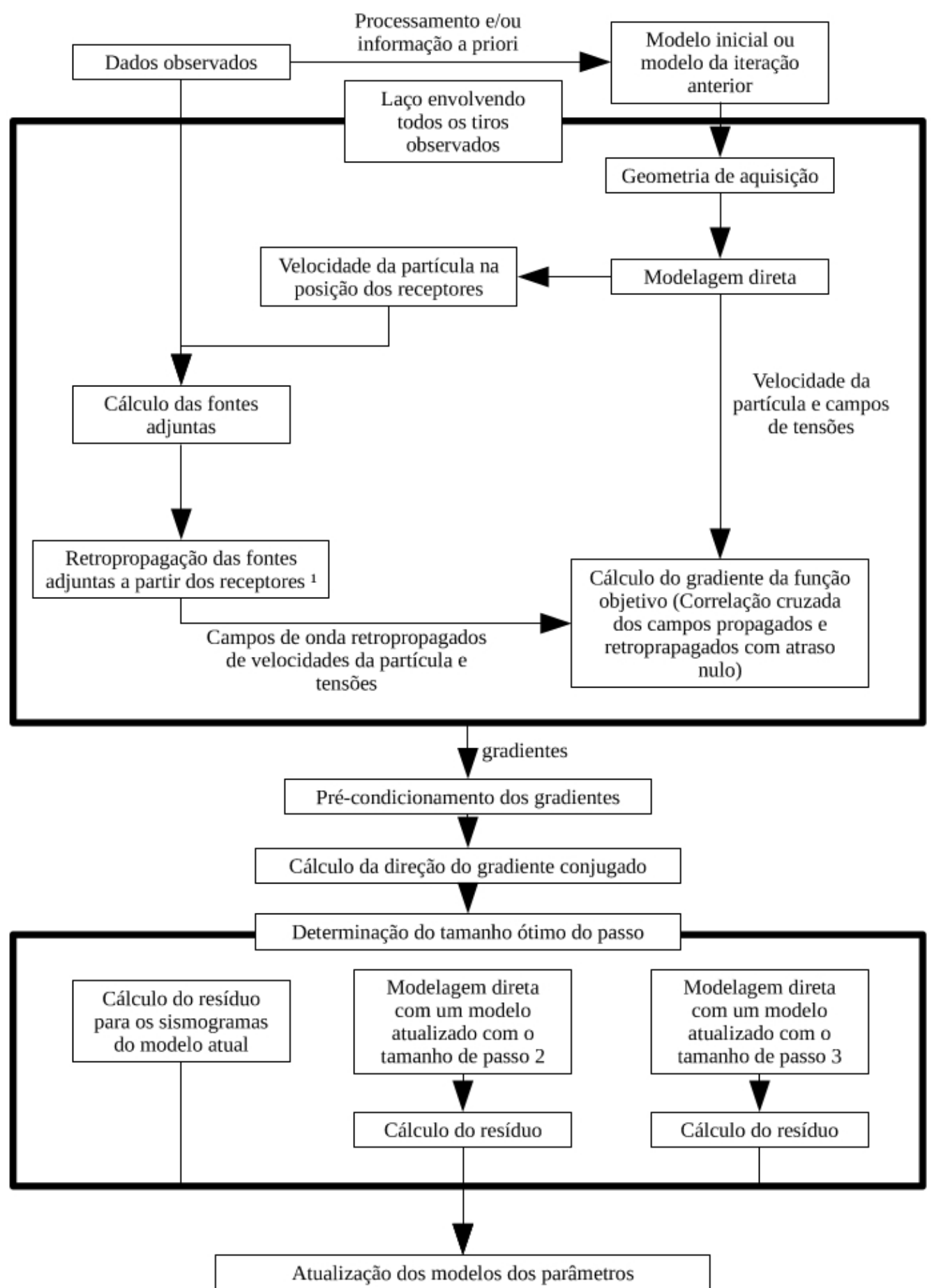

Fonte: Groos (2014). 
O método do gradiente conjudago não linear percorre a direção do gradiente conjugado $\delta c_{n}$ ao invés da direção do gradiente $\delta m_{n}$ e possui uma convergência mais rápida que algoritmos mais simples de descida. Isto pode ser escrito como

$$
\delta c_{n}=\delta m_{n}+\beta_{n} \delta c_{n-1} .
$$

A primeira iteração do algoritmo utiliza a direção de descida mais íngreme:

$$
m_{1}=m_{0}+\alpha \delta m_{0}
$$

Nas demais iterações o modelo é atualizado por

$$
m_{n+1}=m_{n}+\alpha \delta c_{n}
$$

As iterações do algoritmo são calculadas por (3.1) em conjunto com (3.3) e o fator de peso $\beta$ pode ser calculado de diferentes formas. Um exemplo é a forma de Polak-Ribiére utilizada no algoritmo:

$$
\beta_{n}^{P R}=\delta m_{n} \frac{\delta m_{n}-\delta m_{n-1}}{\delta m_{n-1} \delta m_{n-1}}
$$

O tamanho $\alpha$ do passo deve ser calculado antes da atualização do modelo. Para isto, a função objetivo pode ser aproximada localmente por uma parábola com pontos desta parábola obtidos através da adoção de tamanhos diferentes de passos. O tamanho de passo que leva ao mínimo desta parábola é isado como tamanho do passo.

\subsubsection{Estimativa inicial e atualização de parâmetros do modelo}

Existem diferentes estratégias para a obtenção de uma estimativa inicial. $\mathrm{Na}$ sísmica de reflexão rasa pode ser utilizado o processamento convencional aliado a sísmica de refração. No caso da componente radial o processamento dos dados de reflexão da onda convertida de P para S (reflexão PS) assim como a informação fornecida pelas ondas superficiais podem retornar a velocidade da onda S no meio. Em Tran et al. (2013b) é salientada a possibilidade da utilização de modelos obtidos por métodos globais como estimativa inicial.

Como estudado em Karray e Lefebvre (2008) a curva de dispersão é sensível à razão de Poisson que pode ser utilizada para a obtenção da velocidade da onda $\mathrm{P}$ a partir de $V_{S}$. A equação de Poisson pode ser escrita como:

$$
\nu=\frac{1}{2} \frac{\left(V_{P} / V_{S}\right)^{2}-2}{\left(V_{P} / V_{S}\right)^{2}-1} .
$$


Conhecendo o perfil $V_{S}$ é possível realizar uma inversão simples para a razão de Poisson. Restando apenas a densidade. Como este parâmetro apresenta baixa sensibilidade na inversão FWI e também das curvas de dispersão uma alternativa é a utilização da equação de Gardner (GARDNER; GARDNER; GREGORY, 1974) dada por:

$$
\rho=\alpha V_{P}^{\beta}
$$

onde os parâmetros $\alpha$ e $\beta$ na escala de petróleo são determinados a partir de uma curva empírica que determina uma relação entre a velocidade da onda $\mathrm{P}$ e a densidade. Entretanto, em uma escala rasa a relação entre os parâmetros pode divergir do caso determinado em grandes profundidades. Assim estes precisam ser baseados em um conhecimento prévio da região estudada ou em uma forma de estimativa inicial dos parâmetros.

É possível observar ainda que a equação 3.5 pode ser reescrita na forma

$$
V_{P}=V_{s} \sqrt{\frac{\nu-2}{2 \nu-1}}
$$

ou seja, uma constante multiplicando $V_{S}$. Assim, para a atualização dos parâmetros $V_{P}$ e $\rho$ podemos definir as equações

$$
V_{P}=\alpha_{1} V_{S}^{\beta_{1}}+\gamma_{1}
$$

e

$$
\rho=\alpha_{2} V_{P}^{\beta_{2}}+\gamma_{2}
$$

que podem comportar mais tipos de modelos. A

\subsubsection{Gradiente da função objetivo}

Conforme a Figura 4 é necessário obter o gradiente da função objetivo, ou seja, a direção de busca. Este procedimento é realizado fazendo uso da aproximação adjunta. Esta metodologia faz uso da interação entre os campos direto e adjunto através de uma correlação cruzada. Segundo Schäfer (2014) e Tromp, Tape e Liu (2004) esta correlação cruzada de atraso nulo é gerada utilizando a retro-propagação das fontes adjuntas baseadas nos dados, ou seja, os resíduos entre os dados observados e os modelados.

Para simplificar a dedução podemos pensar na atualização do modelo apenas em relação a direção de busca $\delta m_{n}$ de forma que

$$
m_{n+1}=m_{n}+\alpha \delta m_{n}
$$

Assim podemos utilizar a expansão em série de Taylor da energia residual $E\left(m_{n}+\right.$ $\left.\delta m_{n}\right)$ :

$$
E\left(m_{n}+\delta m_{n}\right) \approx E\left(m_{n}\right)+\delta m_{n}\left(\frac{\partial E}{\partial m}\right)_{n}+\frac{1}{2} \delta m_{n}\left(\frac{\partial^{2} E}{\partial m^{2}}\right)_{n} \delta m_{n}^{T}
$$


para encontrar a direção de busca $\delta m_{n}$.

Queremos encontrar o ponto de mínimo da função. Logo, a derivada da equação 3.11 em relação a $\delta m_{n}$ deve ser zero:

$$
\frac{\partial E\left(m_{n}+\delta m_{n}\right)}{\partial \delta m_{n}}=\left(\frac{\partial E}{\partial m}\right)_{n}+\delta m_{n}\left(\frac{\partial^{2} E}{\partial m^{2}}\right)_{n}=0
$$

Isolando $\delta m_{n}$ obtém-se:

$$
\delta m_{n}=-\left(\frac{\partial^{2} E}{\partial m^{2}}\right)_{n}^{-1}\left(\frac{\partial E}{\partial m}\right)_{n}=-H_{n}^{-1}\left(\frac{\partial E}{\partial m}\right),
$$

onde $(\partial E / \partial m)_{n}$ é a direção de descida mais íngreme e $H_{n}^{-1}$ é a matriz Hessiana inversa. A matriz Hessiana usualmente apresenta singularidades e seu cálculo possui um grande peso computacional. Por este motivo no código utilizado esta é aproximada por um operador de pré-condicionamento $P_{n}$. Assim nesta tese foi utilizado o gradiente conjugado da direção de descida de Gauss-Newton dado por 3.13.

Para a estimativa de $\partial E / \partial m$ podemos reescrever a energia residual como:

$$
E=\frac{1}{2} \delta u^{T} \delta u=\frac{1}{2} \sum_{\text {fontes }} \int d t \sum_{\text {receptores }} \delta u^{2}\left(x_{r}, x_{s}, t\right)
$$

Derivando em relação aos parâmetros do modelo obtém-se:

$$
\begin{aligned}
\frac{\partial E}{\partial m} & =\sum_{\text {fontes }} \int d t \sum_{\text {receptores }} \frac{\partial \delta u}{\partial m} \delta u= \\
& =\sum_{\text {fontes }} \int d t \sum_{\text {receptores }} \frac{\partial\left(u^{\text {mod }}-u^{o b s}\right)}{\partial m} \delta u= \\
& =\sum_{\text {fontes }} \int d t \sum_{\text {receptores }} \frac{\partial u^{\text {mod }}(m)}{\partial m} \delta u .
\end{aligned}
$$

Em Köhn (2011) é explicado que pequenas modificações $\delta m$ no espaço dos modelos levam a modificações $\delta \tilde{u}$ no espaço dos dados (FIGURA 5).

Em Tarantola (2005) é mostrado que se a derivada $\partial u / \partial m$ for conhecida, todas as pequenas perturbações do espaço dos modelos podem ser integradas sobre o volume do modelo $(V)$ para o cálculo da mudança provocada no espaço dos dados. A equação seguinte simboliza este processo:

$$
\delta \tilde{u}\left(\mathbf{x}_{\mathbf{s}}, \mathbf{x}_{\mathbf{r}}, t\right)=\hat{L} \delta m:=\int_{V} d V \frac{\partial u}{\partial m} \delta m .
$$

O leitor deve observar que na equação 3.16 foi introduzido o operador linear $\hat{L}$ e que de forma semelhante as mudanças no espaço dos dados podem ser integradas para que a mudança total no espaço dos modelos $\delta m^{\prime}$ possa ser obtida:

$$
\delta m^{\prime}=\hat{L}^{*} \delta \tilde{u}^{\prime}:=\sum_{\text {fontes }} \int d t \sum_{\text {receptores }}\left[\frac{\partial u}{\partial m}\right]^{*} \delta \tilde{u}^{\prime}
$$


Figura 5 - Efeito entre modificações no espaço dos modelos e no espaço dos dados.

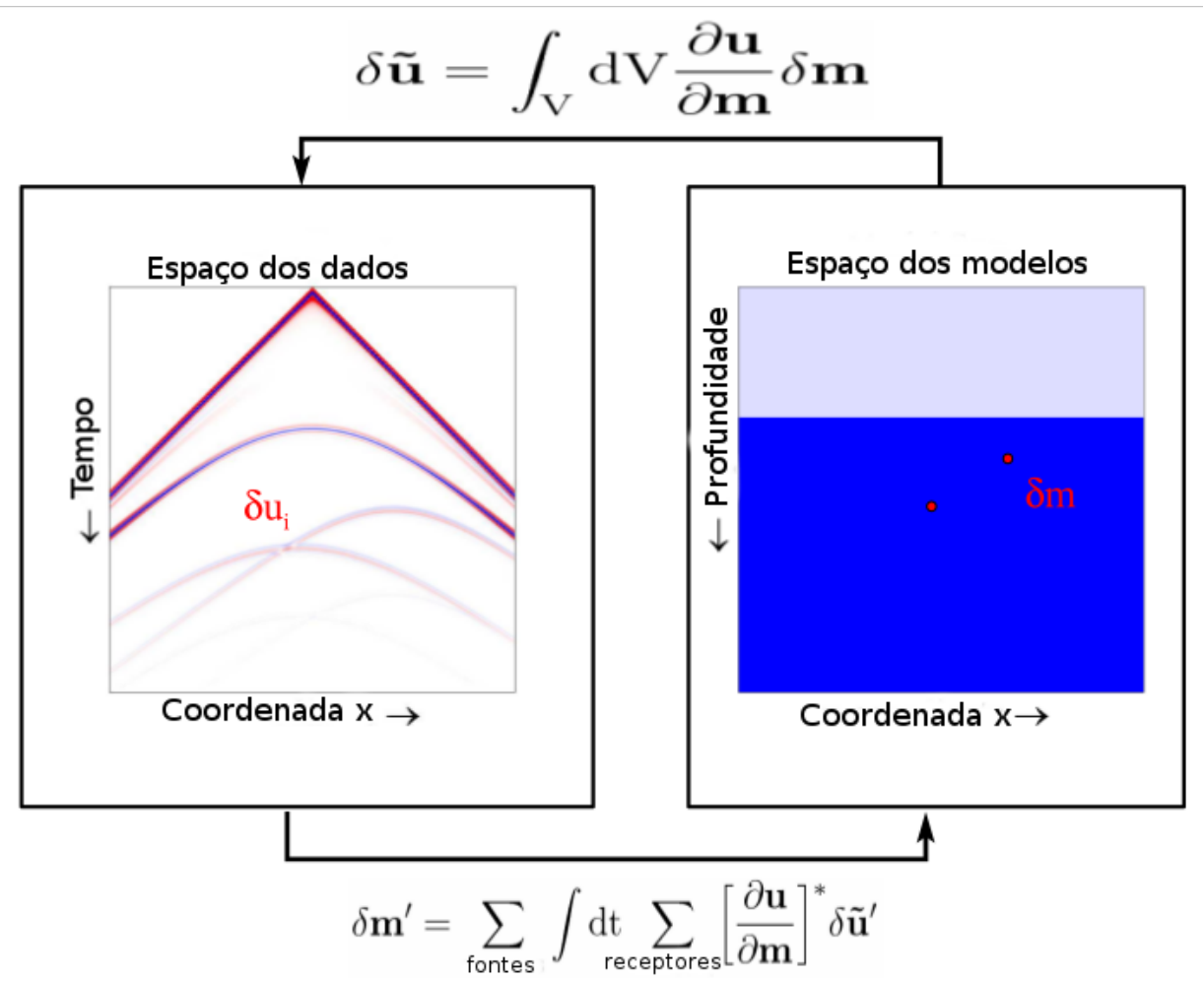

Fonte: Köhn (2011).

Na equação 3.17 a derivada $\partial u / \partial m$ foi substituída por $\partial u^{*} / \partial m$ que é seu adjunto. Entretanto, o operador $\hat{L}$ é linear. Assim, o núcleo de seu adjunto $\hat{L}^{*}$ será idêntico:

$$
\left[\frac{\partial u}{\partial m}\right]^{*}=\left[\frac{\partial u}{\partial m}\right] .
$$

Desta forma, se a pertubação do espaço dos dados $\delta \tilde{u}^{\prime}$ for interpretada como os resíduos dos dados o mapeamento dos dados para o espaço dos modelos é equivalente ao gradiente da energia residual:

$$
\begin{aligned}
\delta m^{\prime} & =\sum_{\text {fontes }} \int d t \sum_{\text {receptores }}\left[\frac{\partial u}{\partial m}\right]^{*} \delta \tilde{u}^{\prime}= \\
& =\sum_{\text {fontes }} \int d t \sum_{\text {receptores }}\left[\frac{\partial u}{\partial m}\right] \delta u= \\
& =\frac{\partial E}{\partial m}
\end{aligned}
$$

Calculando o problema direto $\delta u=\hat{L} \delta m$ e identificando as derivadas de Frechét $\partial u / \partial m$ podemos usar a propriedade de que o operador linear $\hat{L}$ e seu adjunto $\hat{L}^{*}$ possuem os mesmos núcleos. Assim, podemos calcular a direção do gradiente utilizando:

$$
\frac{\partial E}{\partial m}=\partial m^{\prime}=\hat{L}^{*} \delta u^{\prime}
$$

Para obter as derivadas de Frechét podemos aplicar esta ideia nas equações de movimento em um meio elástico. Para que a derivação se torne mais simples o problema 
elástico descrito nas equações 2.2 e 2.3 pode ser reescrito na forma contínua como

$$
\begin{aligned}
& \rho \frac{\partial^{2} u_{i}}{\partial t^{2}}-\frac{\partial \sigma_{i j}}{\partial x_{j}}=f_{i}, \\
& \sigma_{i j}-c_{i j k l} \epsilon_{k l}=T_{i j}, \\
& \epsilon_{i j}=\frac{1}{2}\left(\frac{\partial u_{i}}{\partial x_{j}}+\frac{\partial u_{j}}{\partial x_{i}}\right),
\end{aligned}
$$

onde também devem ser levadas em conta as condições iniciais e de borda e $\epsilon_{i j}$ é o tensor de deformação, $c_{i j k l}$ é o tensor de rigidez, $f_{i}$ e $T_{i j}$ são os termos para as forças de volume e superfície, respectivamente.

Perturbando cada parâmetro e variável de 3.21 por uma pertubação de primeira ordem temos:

$$
\begin{aligned}
& u_{i} \rightarrow u_{i}+\delta u_{i}, \\
& \rho \rightarrow \rho+\delta \rho, \\
& \sigma_{i j} \rightarrow \sigma_{i j}+\delta \sigma_{i j}, \\
& c_{i j k l} \rightarrow c_{i j k l}+\delta c_{i j k l}, \\
& \epsilon_{i j} \rightarrow \epsilon_{i j}+\delta \epsilon_{i j}
\end{aligned}
$$

As equações 3.22 levam a novas equações de movimento:

$$
\begin{aligned}
& \rho \frac{\partial^{2} \delta u_{i}}{\partial t^{2}}-\frac{\partial \delta \sigma_{i j}}{\partial x_{j}}=\Delta f_{i}, \\
& \delta \sigma_{i j}-c_{i j k l} \delta \epsilon_{k l}=\Delta T_{i j}, \\
& \delta \epsilon_{i j}=\frac{1}{2}\left(\frac{\partial \delta u_{i}}{\partial x_{j}}+\frac{\partial \delta u_{j}}{\partial x_{i}}\right),
\end{aligned}
$$

onde

$$
\Delta f_{i}=-\delta \rho \frac{\partial^{2} u_{i}}{\partial t^{2}}
$$

e

$$
\Delta T_{i j}=\delta c_{i j k l} \epsilon_{k l}
$$

A função elástica de Green $G_{i j}\left(\mathbf{x}, t ; \mathbf{x}^{\prime}, t^{\prime}\right)$ pode ser utilizada na obtenção da solução das equações elásticas perturbadas 3.23. Aplicando esta função obtemos:

$$
\begin{array}{r}
\delta u_{i}(\mathbf{x}, t)=\int_{V} d V \int_{0}^{T} d t^{\prime} G_{i j}\left(\mathbf{x}, t ; \mathbf{x}^{\prime}, t^{\prime}\right) \Delta f_{j}\left(\mathbf{x}^{\prime}, t\right) \\
-\int_{V} d V \int_{0}^{T} d t^{\prime} \frac{\partial G_{i j}}{\partial x_{k}^{\prime}}\left(\mathbf{x}, t ; \mathbf{x}^{\prime}, t^{\prime}\right) \Delta T_{j k}\left(\mathbf{x}^{\prime}, t^{\prime}\right)
\end{array}
$$

Substituindo em 3.26 as equações 3.24 e 3.25 temos:

$$
\begin{gathered}
\delta u_{i}(\mathbf{x}, t)=-\int_{V} d V \int_{0}^{T} d t^{\prime} G_{i j}\left(\mathbf{x}, t ; \mathbf{x}^{\prime}, t^{\prime}\right) \frac{\partial^{2} u_{j}}{\partial t^{2}}\left(\mathbf{x}^{\prime}, t^{\prime}\right) \delta \rho \\
-\int_{V} d V \int_{0}^{T} d t^{\prime} \frac{\partial G_{i j}}{\partial x_{k}^{\prime}}\left(\mathbf{x}, t ; \mathbf{x}^{\prime}, t^{\prime}\right) \epsilon_{l m}\left(\mathbf{x}^{\prime}, t^{\prime}\right) \delta c_{j k l m} .
\end{gathered}
$$

Introduzindo isotropia o tensor de rigidez em 3.27 ganha nova forma levando a equação: 


$$
\begin{aligned}
& \delta u_{i}(\mathbf{x}, t)=-\int_{V} d V\left[\int_{0}^{T} d t^{\prime} G_{i j}\left(\mathbf{x}, t ; \mathbf{x}^{\prime}, t^{\prime}\right) \frac{\partial^{2} u_{j}}{\partial t^{2}}\left(\mathbf{x}^{\prime}, t^{\prime}\right)\right] \delta \rho \\
& -\int_{V} d V\left[\int_{0}^{T} d t^{\prime} \frac{\partial G_{i j}}{\partial x_{k}^{\prime}}\left(\mathbf{x}, t, \mathbf{x}^{\prime}, t^{\prime}\right) \epsilon_{l m}\left(\mathbf{x}^{\prime}, t^{\prime}\right) \delta_{j k} \delta_{l m}\right] \delta \lambda \\
& -\int_{V} d V\left[\int_{0}^{T} d t^{\prime} \frac{\partial G_{i j}}{\partial x_{k}^{\prime}}\left(\mathbf{x}, t ; \mathbf{x}^{\prime}, t^{\prime}\right) \epsilon_{l m}\left(\mathbf{x}^{\prime}, t^{\prime}\right)\left(\delta_{j l} \delta_{l m}+\delta_{j m} \delta_{k l}\right] \delta \mu .\right.
\end{aligned}
$$

A equação 3.28 tem a mesma forma da equação 3.16, desejada para a solução do problema direto. Desta forma, os núcleos de Frechét $\partial u_{i} / \partial \mathbf{m}(\mathbf{x})$ individuais de cada parâmetro podem ser identificados:

$$
\begin{aligned}
\frac{\partial u_{i}}{\partial \rho} & =-\int_{0}^{T} d t^{\prime} G_{i j}\left(\mathbf{x}, t ; \mathbf{x}^{\prime}, t^{\prime}\right) \frac{\partial^{2} u_{j}}{\partial t^{2}}\left(\mathbf{x}^{\prime}, t^{\prime}\right), \\
\frac{\partial u_{i}}{\partial \lambda} & =-\int_{0}^{T} d t^{\prime} \frac{\partial G_{i j}}{\partial x_{k}^{\prime}}\left(\mathbf{x}, t ; \mathbf{x}^{\prime}, t^{\prime}\right) \epsilon_{l m}\left(\mathbf{x}^{\prime}, t^{\prime}\right) \delta_{j k} \delta_{l m}, \\
\frac{\partial u_{i}}{\partial \mu} & =-\int_{0}^{T} d t^{\prime} \frac{\partial G_{i} j}{\partial x_{k}^{\prime}}\left(\mathbf{x}, t ; \mathbf{x}^{\prime}, t^{\prime}\right) \epsilon\left(\mathbf{x}^{\prime}, t^{\prime}\right)\left(\delta_{j l} \delta_{l m}+\delta_{j m} \delta_{k l}\right)
\end{aligned}
$$

O adjunto da equação 3.16 pode ser escrito como:

$$
\delta \mathbf{m}^{\prime}(\mathbf{x})=\sum_{\text {fontes }} \int_{0}^{T} d t \sum_{\alpha=1}^{N_{\text {fontes }}}\left[\frac{\partial u_{i}}{\partial \mathbf{m}}\right]^{*} \delta u_{i}^{\prime}\left(\mathbf{x}_{\alpha}, t^{\prime}\right)
$$

É possível inserir os núcleos integrais de 3.29 em 3.30 já que um operador linear e sua transposta possuem os mesmos núcleos $\partial u_{i} / \partial \mathbf{m}$.

$$
\begin{aligned}
& \delta \rho^{\prime}=-\sum_{\text {fontes }} \int_{0}^{T} d t \sum_{\alpha=1}^{N_{\text {receptores }}} \int_{0}^{T} d t^{\prime} G_{i j}\left(\mathbf{x}_{\alpha}, t^{\prime} ; \mathbf{x}, t\right) \frac{\partial^{2} u_{j}}{\partial t^{2}}(\mathbf{x}, t) \delta u_{i}^{\prime}\left(\mathbf{x}_{\alpha}, t^{\prime}\right) \\
& \delta \lambda^{\prime}=-\sum_{\text {fontes }} \int_{0}^{T} d t \sum_{\alpha=1}^{N_{\text {receptores }}} \int_{0}^{T} d t^{\prime} \frac{\partial G_{i j}}{\partial x_{k}}\left(\mathbf{x}_{\alpha}, t^{\prime} ; \mathbf{x}, t\right) \epsilon_{l m}(\mathbf{x}, t) \delta_{j k} \delta_{l m} \delta u_{i}^{\prime}\left(\mathbf{x}_{\alpha}, t^{\prime}\right) \\
& \delta \mu^{\prime}=-\sum_{\text {fontes }} \int_{0}^{T} d t \sum_{\alpha=1}^{N_{\text {receptores }}^{T}} \int_{0}^{T} d t^{\prime} \frac{\partial G_{i j}}{\partial x_{k}}\left(\mathbf{x}_{\alpha}, t^{\prime} ; \mathbf{x}, t\right) \epsilon_{l m}(\mathbf{x}, t)\left(\delta_{j l} \delta_{l m}+\delta_{j m} \delta_{k l}\right) \delta u_{i}^{\prime}\left(\mathbf{x}_{\alpha}, t^{\prime}\right)
\end{aligned}
$$

Movendo os termos que dependem somente do tempo $t$ e das posições $x$ para fora da somatória sobre os receptores:

$$
\begin{aligned}
& \delta \rho^{\prime}=-\sum_{\text {fontes }} \int_{0}^{T} d t \frac{\partial^{2} u_{j}}{\partial t^{2}}(\mathbf{x}, t) \sum_{\alpha=1}^{N_{\text {receptores }}} \int_{0}^{T} d t^{\prime} G_{i j}\left(\mathbf{x}_{\alpha}, t^{\prime} ; \mathbf{x}, t\right) \delta u_{i}^{\prime}\left(x_{\alpha}, t^{\prime}\right) \\
& \delta \lambda^{\prime}=-\sum_{\text {fontes }} \int_{0}^{T} d t \epsilon_{l m}(\mathbf{x}, t) \delta_{j k} \delta_{l m} \sum_{\alpha}^{N_{\text {receptores }}} \int_{0}^{T} d t^{\prime} \frac{\partial G_{i j}}{\partial x_{k}}\left(\mathbf{x}_{\alpha}, t^{\prime} ; \mathbf{x}, t\right) \delta u_{i}^{\prime}\left(\mathbf{x}_{\alpha}, t^{\prime}\right) \\
& \delta \mu^{\prime}=-\sum_{\text {fontes }} \int_{0}^{T} d t \epsilon_{l m}(\mathbf{x}, t)\left(\delta_{j l} \delta_{l m}+\delta_{j m} \delta_{k l}\right) \sum_{\alpha=1}^{N_{\text {receptores }}} \int_{0}^{T} d t^{\prime} \frac{\partial G_{i j}}{\partial x_{k}}\left(\mathbf{x}_{\alpha}, t^{\prime} ; \mathbf{x}, t\right) \delta u_{i}^{\prime}\left(\mathbf{x}_{\alpha}, t^{\prime}\right)
\end{aligned}
$$

e definindo o campo de onda

$$
\Psi_{j}(\mathbf{x}, t)=\sum_{\alpha=1}^{N_{\text {receptores }}} \int_{0}^{T} d t^{\prime} G_{i j}\left(\mathbf{x}_{\alpha}, t^{\prime}, \mathbf{x}, t\right) \delta u_{i}^{\prime}\left(\mathbf{x}_{\alpha}, t^{\prime}\right)
$$


podemos reescrever a equação 3.31 como

$$
\begin{aligned}
& \delta \rho^{\prime}=-\sum_{\text {fontes }} \int_{0}^{T} d t \frac{\partial^{2} u_{j}}{\partial t^{2}}(\mathbf{x}, t) \Psi_{j}, \\
& \delta \lambda^{\prime}=-\sum_{\text {fontes }} \int_{0}^{T} d t \epsilon_{l m}(\mathbf{x}, t) \delta_{j k} \delta_{l m} \frac{\partial \Psi_{j}}{\partial x_{k}}, \\
& \delta \lambda^{\prime}=-\sum_{\text {fontes } 0} \int_{0}^{T} d t \epsilon_{l m}(\mathbf{x}, t)\left(\delta_{j l} \delta_{l m}+\delta_{j m} \delta_{k l}\right) \frac{\partial \Psi_{j}}{\partial x_{k}}
\end{aligned}
$$

Para obter uma expressão com uma simetria maior em relação as equações dos demais parâmetros que a equação 3.34 é possível integrar o gradiente de densidade por partes

$$
\delta \rho^{\prime}=-\sum_{\text {fontes }}\left\{\left[\frac{\partial u_{j}}{\partial t}(\mathbf{x}, T) \Psi_{j}(\mathbf{x}, T)\right]_{0}^{T}-\int_{0}^{T} d t \frac{\partial u_{j}}{\partial t}(\mathbf{x}, T) \frac{\partial \Psi_{j}}{\partial t}(\mathbf{x}, T)\right\} .
$$

O campo $u_{j}(\mathbf{x}, t)$ satisfaz as condições de repouso, $u_{j}(\mathbf{x}, 0)=0$ e $\partial u_{j}(\mathbf{x}, 0) / \partial t=0$. E o campo $\Psi_{j}(\mathbf{x}, t)$ satisfaz a condição de repouso final $\Psi_{j}(\mathbf{x}, T)=0$. Desta forma,

$$
\delta \rho^{\prime}=\sum_{\text {fontes }} \int_{0}^{T} d t \frac{\partial u_{j}}{\partial t}(\mathbf{x}, t)
$$

Retornando aos gradientes dos parâmetros de Lamé $\delta \lambda^{\prime}$ e $\delta \mu^{\prime}$ e explicitando as somas implícitas dos gradientes temos:

$$
\begin{aligned}
& \delta \lambda^{\prime}=-\sum_{\text {fontes }} \int_{0}^{T} d t \sum_{l} \sum_{k} \sum_{j} \sum_{m} \epsilon_{l m}(\mathbf{x}, t) \delta_{j k} \delta_{l m} \frac{\partial \Psi_{j}}{\partial x_{k}} \\
& \delta \mu^{\prime}=-\sum_{\text {fontes }} \int_{0}^{T} d t \sum_{l} \sum_{k} \sum_{j} \sum_{m} \epsilon_{l m}(\mathbf{x}, t)\left(\delta_{j l} \delta_{l m}+\delta_{j m} \delta_{k l}\right) \frac{\partial \Psi_{j}}{\partial x_{k}}
\end{aligned}
$$

E negligenciando todas as componentes do campo de onda e derivadas na direção $y$ temos:

$$
\begin{aligned}
& \delta \lambda^{\prime}=-\sum_{\text {fontes }} \int_{0}^{T} d t\left(\epsilon_{x x}+\epsilon_{z z}\right)\left(\frac{\partial \Psi_{x}}{\partial x}+\frac{\partial \Psi_{z}}{\partial z}\right), \\
& \delta \mu^{\prime}=-\sum_{\text {fontes } 0} \int_{0}^{T} d t\left[\left(\epsilon_{x z}+\epsilon_{z x}\right)\left(\frac{\partial \Psi_{x}}{\partial z}+\frac{\partial \Psi_{z}}{\partial x}\right)\right]+2\left(\epsilon_{x x} \frac{\partial \Psi_{x}}{\partial x}+\epsilon_{z z} \frac{\partial \Psi_{z}}{\partial z}\right) .
\end{aligned}
$$

Utilizando a definição do tensor de deformação e colocando em conjunto o gradiente para a densidade podemos reescrever:

$$
\begin{aligned}
\delta \lambda^{\prime} & =-\sum_{\text {fontes }} \int_{0}^{T} d t\left(\frac{\partial u_{x}}{\partial x}+\frac{\partial u_{z}}{\partial z}\right)\left(\frac{\partial \Psi_{x}}{\partial x}+\frac{\partial \Psi_{z}}{\partial z}\right) \\
\delta \mu^{\prime} & =-\sum_{\text {fontes }} \int_{0}^{T} d t\left[\left(\frac{\partial u_{x}}{\partial z}+\frac{\partial u_{z}}{\partial x}\right)\left(\frac{\partial \Psi_{x}}{\partial z}+\frac{\partial \Psi_{z}}{\partial x}\right)\right]+2\left(\frac{\partial u_{x}}{\partial x} \frac{\partial \Psi_{x}}{\partial x}+\frac{\partial u_{z}}{\partial z} \frac{\partial \Psi_{z}}{\partial z}\right), \\
\delta \rho^{\prime} & =\sum_{\text {fontes }} \int d t\left(\frac{\partial u_{x}}{\partial t} \frac{\partial \Psi_{x}}{\partial t}+\frac{\partial u_{z}}{\partial t} \frac{\partial \Psi_{z}}{\partial t}\right)
\end{aligned}
$$


Em Köhn et al. (2012) diferentes tipos de parametrização foram estudados. Como conclusão uma parametrização em termos da velocidades e densidade gera resultados mais precisos em aquisições de afastamentos longos. E ao mesmo tempo este tipo de parametrização retornou bons resultados em Nuber, Manukyan e Maurer (2015) e em Groos et al. (2017). Para adotar esta parametrização podemos usar as relações:

$$
\begin{aligned}
& V_{P}=\sqrt{\frac{\lambda+2 \mu}{\rho}}, \\
& V_{S}=\sqrt{\frac{\mu}{\rho}} .
\end{aligned}
$$

Assim, devemos aplicar a regra da cadeia em termos dos novos parâmetros dos modelos $m_{\text {novo }}$ sobre o núcleo de Frechét no problema adjunto dado pela equação 3.30:

$$
\delta m_{\text {novo }}=\sum_{\text {fontes }} \int d t \sum_{R}\left[\frac{\partial u}{\partial m} \frac{\partial m}{\partial m_{\text {novo }}}\right]^{*} \delta u
$$

De forma que o gradiente para $V_{P}$ pode ser escrito como:

$$
\begin{aligned}
\delta V_{p} & =\sum_{\text {fontes }} d t \sum_{R}\left[\frac{\partial u}{\partial \lambda} \frac{\partial \lambda}{\partial V_{P}}+\frac{\partial u}{\partial \mu} \frac{\partial \mu}{\partial V_{P}}+\frac{\partial u}{\partial \rho} \frac{\partial \rho}{\partial V_{P}}\right]^{*} \delta u_{i}= \\
& =\sum_{\text {fontes }} \int d t \sum_{R}\left[\frac{\partial u}{\partial \lambda} 2 \rho V_{P}\right]^{*} \delta u_{i}= \\
& =2 \rho V_{P} \sum_{\text {fontes }} \int d t \sum_{R}\left[\frac{\partial u}{\partial \lambda}\right]^{*} \delta u_{i}= \\
& =2 \rho V_{P} \delta \lambda .
\end{aligned}
$$

Consequentemente o conjunto de equações para todos os parâmetros fica:

$$
\begin{aligned}
& \delta V_{P}=2 \rho V_{P} \delta \lambda \\
& \delta V_{S}=-4 \rho V_{S} \delta \lambda+2 \rho V_{S} \delta \mu \\
& \delta \rho_{v e l}=\left(V_{P}^{2}-2 V_{S}^{2}\right) \delta \lambda+V_{S}^{2} \delta \mu+\delta \rho .
\end{aligned}
$$

\subsubsection{Norma L2 e fonte adjunta correspondente}

Assim como em Groos et al. (2017) aqui foi resolvido um problema de mínimos quadrados:

$$
E=\frac{\sum_{i}^{n_{s}} \sum_{j}^{n_{r}} \sum_{k}^{n_{c}}\left|u_{i, j, k}^{\text {mod }}-\hat{u}_{i, j, k}^{o b s}\right|^{2}}{n_{s} n_{r} n_{c}}
$$

onde as somas denotam respectivamente as somas sobre as $n_{s}$ fontes, $n_{r}$ receptores e $n_{c}$ componentes. Segundo Groos et al. (2017) esta norma possui vantagens já que não é sensível a decaimentos na amplitude puramente dependentes da distância em relação à fonte como o decaimento causado por espalhamento geométrico e efeitos anelásticos quando ondas com um único e fixo comprimento de onda. Da mesma forma, esta norma não apresenta sensibilidade a variações de amplitude causadas por acoplamento dos receptores. 
Porém diferenças nas amplitudes causadas por decaimento de amplitude dependentes de frequências devido a atenuação anelástica ou da transformação 3D/2D (próximo tópico), ainda influenciam o valor da norma.

\subsubsection{Correção 3D/2D}

Em Schäfer (2014). Forbriger, Groos e Schafer (2014) e Schafer et al. (2014) foram estudados os efeitos 3D da propagação de ondas em aplicações 2D de FWI. Groos et al. (2014b) explica que quando um campo de ondas é excitado, por uma marreta no solo por exemplo, esta fonte representa uma fonte pontual e para comparar este tipo com dados 2D sintéticos deve ser feita uma correção nos dados reais. Quando é realizada uma modelagem 2D como a apresentada no capítulo 2 , as fontes atuam implicitamente como fontes lineares.

Aqui, assim como em Groos et al. (2014b), foi adotada uma transformação 3D/2D introduzida por Schafer et al. (2014) como transformação de onda-direta que deve ser realizada sobre os dados reais antes da aplicação da FWI. Esta transformação realizada em três passos.

1. Cada sismograma é convolvido com $t^{-1 / 2}$.

2. Após isso cada sismograma é cortado com $t^{-1 / 2}$.

3. E cada sismograma é escalado com $r \sqrt{2 / t}$.

Nestes passos $t$ é o tempo de percurso e $r$ é o afastamento do receptor. Em Schafer et al. (2014) foi mostrado que esta aproximação funciona para o caso elástico em estruturas 2D, como exceção das ondas retro propagadas.

\subsubsection{Salto de ciclos}

Tanto o modelo inicial quando a wavelet inicial fornecidas para a FWI desempenham um papel importante. Segundo Groos (2013) para que ocorra convergência para o ponto de mínimo as formas de onda geradas através da modelagem devem se ajustar aos dados observados com menos de meio período de diferença. Caso este requisito não seja cumprido ocorrerá o efeito de salto de ciclo e a inversão irá convergir para um mínimo local. O código DENISE apresenta a possibilidade de análise de pequenas partes do dado com diferentes intervalos de frequência Este processo é realizado através de um filtro de Butterworth passa-baixa a cada número pré-fixado de iterações. Bunks et al. (1995) mostra que isto diminui os pré-requisitos para que não ocorra o salto de ciclo. 
Figura 6 - Fontes em uma aquisição sísmica. As setas representam as fontes e os triângulos os geofones. Numa aquisição real temos apenas a seta vermelha (fonte pontual). Numa modelagem sintética existe fonte linear (setas azuis) ao longo do eixo perpendicular ao do modelo.

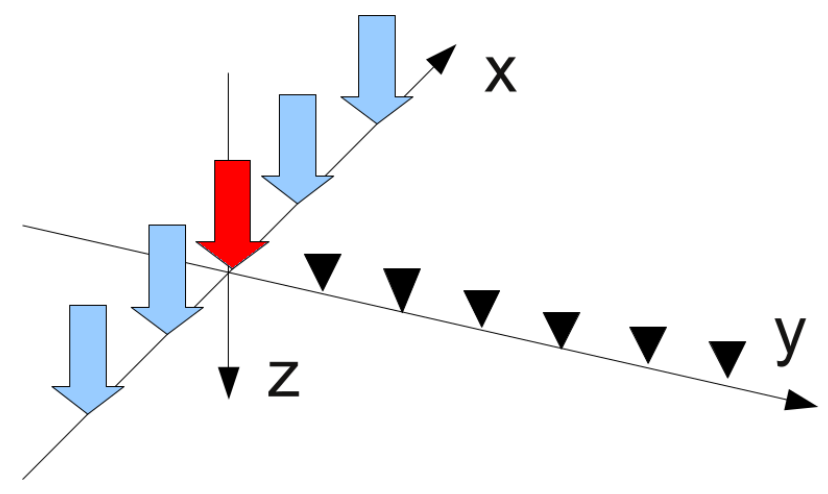

Fonte: Schäfer (2014).

\subsubsection{Operador de pré-condicionamento}

O operador de pré-condicionamento funciona como uma aproximação da Hessiana (EQUAÇÃO 3.13). No código IFOS2D existem duas aproximações diferentes que podem ser utilizadas.

A primeira aproximação, utilizada em Shin et al. (2001), é uma aproximação da Hessiana que pode ser obtida por

$$
H_{a}=J^{T} J
$$

onde, $J=\partial u / \partial m . \quad$ Assumindo que o gradiente $\partial E / \partial m$ pode ser reescrito

$$
\frac{\partial E}{\partial m}=\frac{\partial}{\partial m}\left(\frac{1}{2} \delta u^{T} \delta u\right)=J^{T} \delta u
$$

Podemos reescrever a equação 3.13 na forma

$$
\delta m=\left(\operatorname{diag} H_{a}+\lambda I\right)^{-1} J^{T} \delta u
$$

onde foi incluído um fator de amortecimento $\lambda$ (este lambda não possui relação com o parâmetro de Lamé).

A segunda aproximação é baseada no trabalho de Plessix e Mulder (2004). Esta também faz uso da equação 3.47, porém a aproximação da Hessiana é calculada por:

$$
H_{a}=\sqrt{W_{S}} *\left(\operatorname{asenh}\left(\frac{x \max -x}{z}\right)-\operatorname{asenh}\left(\frac{x \min -x}{z}\right)\right)
$$

onde $W_{S}$ é o campo de onda modelado e os termos entre parênteses representam uma aproximação da função de Green dos receptores. 


\subsection{Busca global por evolução diferencial}

Em Storn e Price (1997) foi demonstrada a rápida convergência do algoritmo de evolução diferencial (ED) em comparação a outros métodos de busca global. Assim como no caso da busca local os sismogramas sintéticos são comparados com os sismogramas observados através de uma norma. A evolução diferencial foi utilizada neste trabalho como uma forma de analisar o problema. Portanto, para a aplicação deste algoritmo também foi utilizada a norma dada pela equação 3.44.

A ED cai na mesma categoria dos algoritmos genéticos e desta forma utiliza processos como mutação, cruzamento e seleção. Para casa geração $G$ este método usa $N P$ vetores de parâmetros com dimensão $D$

$$
x_{i, G} \quad \text { com } \quad i=1,2, \ldots N P
$$

onde o valor NP não muda durante a minimização.

Nesta aplicação de ED foi utilizado o método de Latin Hypercube ??) para a criação da população inicial e maximização da cobertura do espaço de busca. Os tópicos de mutação, cruzamento e seleção do algoritmo podem ser descritos da seguinte norma:

- Mutação: A mutação gera vetores mutantes para cada vetor de parâmetros fazendo uso da regra:

$$
q_{i, G+1}=x_{r 1, G}+F\left(x_{r 2, G-x_{r 3, G}}\right)
$$

onde os índices aleatórios $r 1, r 2, r 3 \in\{1,2, \ldots, N P\}$ são inteiros, mutualmente diferentes e $\operatorname{Fin}[0,2]$ é um fator real e constante que controla a perturbação de um vetor $x_{r 1, G}$.

- Cruzamento: Neste processo um novo vetor tentativa $p_{, G+1}$ é criado a partir dos elementos do vetor competidor $x_{i, G}$ e do vetor mutante $q_{i, G+1}$. Este processo melhora a diversidade dos elementos de um geração. $\mathrm{O}$ vetor tentativa

$$
p_{i, G+1}=\left(p_{1 i, G+1} \cdot p_{2 i, G+1}, \ldots, p_{d i, G+1}\right)
$$

é construído seguindo a regra

$$
p_{j i, G+1}=\left\{\begin{array}{cccc}
q_{j i, G+1} & \text { se } \quad(\operatorname{randb}(j)<=C R) & \text { ou } j=\operatorname{rnbr}(i), \\
x_{j i, G} & \text { se } & (\operatorname{randb}(j)>C R) & \text { e } j \neq \operatorname{rnbr}(i)
\end{array} \quad j=1,2, \ldots D .\right.
$$

O termo $\operatorname{randb}(j)$ em 3.52 é a j-ésima avaliação de um gerador uniforme aleatório com saída no intervalo $[0,1]$. O termoCR é a constante de cruzamento que deve ser 
fornecida pelo usuário. $\operatorname{rnbr}(i)$ é um índice escolhido aleatoriamente no intervalo $1,2, \ldots \mathrm{D}$ que garante que $p_{i, G+1}$ tenha apenas um parâmetro de $q_{i, G+1}$.

- Seleção: A seleção é o processo que decide quais membros de uma geração $G$ irão passar para a próxima geração $G+1$. O vetor tentativa é comparado a um vetor alvo considerando a função custo. Os vetores com menor valor da função custo/objetivo são passados para a próxima geração.

\subsection{Estimativa do fator de qualidade Q}

Existem diferentes formas de estimar o fator de qualidade. Em Cheng e Margrave (2013) são citados os métodos do sinal analítico, o de razão espectral de Bath (1974), o de frequência de deslocamento centroide de Quan e Harris (1997), a técnica de partida e o método de modelagem espectral estudado em Balis (2011).

O método de frequência de deslocamento centroide possui aplicabilidade em dados de reflexão. Sendo $\left|A_{1}(f)\right|$ o espectro de amplitude de um sinal próximo da fonte e $\left|A_{2}(f)\right|$ o de um sinal mais afastado da fonte. Assim a frequência centroide pode ser escrita como

$$
f_{c 1}=\frac{\int_{0}^{\infty} f\left|A_{1}(f)\right| d f}{\int_{0}^{\infty}\left|A_{1}(f)\right| d f},
$$

sendo que a variância é dada por

$$
\sigma_{1}^{2}=\frac{\int_{0}^{\infty}\left(f-f_{c 1}\right)^{2}\left|A_{1}(f)\right| d f}{\int_{0}^{\infty}\left|A_{1}(f)\right| d f} .
$$

A frequência centroide e a variância do sinal mais afastado da fonte podem ser escritas da mesma forma que em 3.53 e 3.54 substituindo os termos $\left|A_{1}(f)\right|$ e $f_{c 1}$ por $\left|A_{2}(f)\right|$ e $f_{c 2}$ respectivamente.

A componente de alta frequência possui atenuação preferencial e por isso ocorre uma movimentação da frequência centroide. Esta movimentação está ligada a atenuação $Q$ por

$$
\int_{\text {raio }} \frac{\pi}{Q \nu} d l=\frac{\pi \tau}{Q_{\text {inter }}}
$$

sendo que $Q_{\text {inter }}$ é o $Q$ intervalar correspondente ao tempo de percurso $\tau$ para este processo de atenuação. Com isto, o $Q$ pode ser estimado através de

$$
Q_{e s t}=\frac{\pi \tau \sigma_{1}^{2}}{f_{c 1}-f_{c 2}} .
$$


Esta última equação mostra que o Q estimado é proporcional a movimentação da frequência $f_{c 1}-f_{c 2}$ e a variância $\sigma_{1}^{2}$. 


\section{Avaliação em Dados Sintéticos}

Neste capítulo foram avaliadas as estimativas de parâmetros dos meio através de modelos simplificados. Também foram simulados dois levantamentos CMP de reflexão para melhor comparação com os resultados dos estudos de casos reais apresentados nos capítulos seguintes.

\subsection{Avaliação pela estratégia de otimização global de parâmetros}

Para iniciar a avaliação do problema foi aplicada uma estratégia estocástica de inversão que permite a visualização do comportamento da função objetivo considerando-se a variação de todos os parâmetros. Este tipo de procedimento foi aplicado em Bokhonok, Diogo e Prado (2013), Bokhonok et al. (2015) e Spadini et al. (2015).

Neste trabalho foi utilizada a metodologia de busca por evolução diferencial. O procedimento foi aplicado duas vezes utilizando populações iniciais aleatórias de forma que os valores avaliados por cada realização da busca fossem diferentes. Os pontos do espaço de parâmetros do modelo nas iterações finais do algoritmo de evolução diferencial se aglomeram em torno dos pontos de mínimo da função objetivo que são "vistos"pelo algoritmo como os mais favoráveis. Resultados do mesmo tipo são apresentados em Fliedner, Treitel e MacGregor (2012) na aplicação da FWI através de um algoritmo de vizinhança.

Para complementar o estudo da função objetivo também foi realizada uma análise da sensibilidade da norma L2 em relação a cada parâmetro.

Para a simulação do dado observado foi utilizado um modelo característico da escala rasa no mapeamento do embasamento (TABELA 1), considerando camadas isotrópicas com interfaces planas. A resposta buscada também possui este caráter 1D.

Os sismogramas de componentes vertical e radial, Figuras 7 e 8, respectivamente, foram modelados simultaneamente. A modelagem foi realizada simulando uma fonte vertical com uma wavelet de Ricker de $51.25 \mathrm{~Hz}$. Para estes primeiros testes a simulação foi do tipo elástica. A intenção era observar a inversão de parâmetros em uma situação ideal.

O estudo do comportamento da função objetivo foi realizado simulando duas janelas

Tabela 1 - Propriedades físicas do modelo de camadas planas utilizado para a simulação do dado observado.

\begin{tabular}{ccccc}
\hline Camada & $V_{P}(\mathrm{~m} / \mathrm{s})$ & $V_{S}(\mathrm{~m} / \mathrm{s})$ & $\rho\left(\mathrm{kg} / \mathrm{m}^{3}\right)$ & Profundidade $(\mathrm{m})$ \\
1 & 370 & 200 & 1530 & - \\
2 & 1650 & 312 & 1910 & 5 \\
3 & 3000 & 1680 & 2540 & 20 \\
\hline
\end{tabular}


de afastamentos diferentes, com 58 receptores cada. A primeira com afastamentos de 1 a 48 metros e a segunda janela de 49 a 96 metros ( apenas afastamentos longos em relação a profundidade dos refletores (TABELA 1)).

Figura 7 - Conjunto de tiro de componente vertical criado a partir dos dados da Tabela 1.

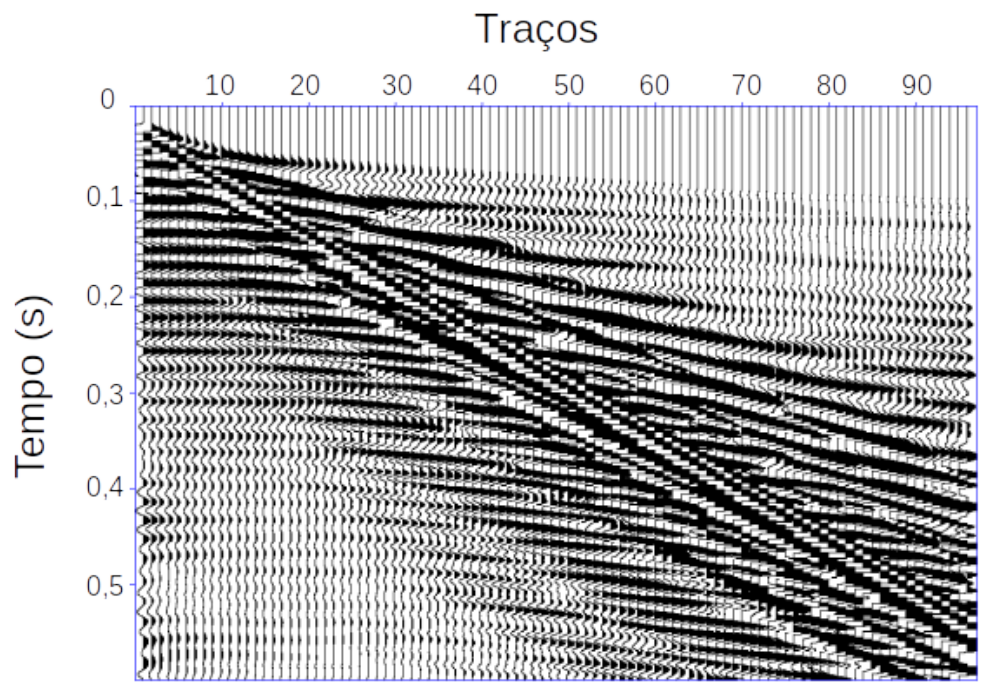

Figura 8 - Conjunto de tiro de componente radial criado a partir dos dados da Tabela 1.

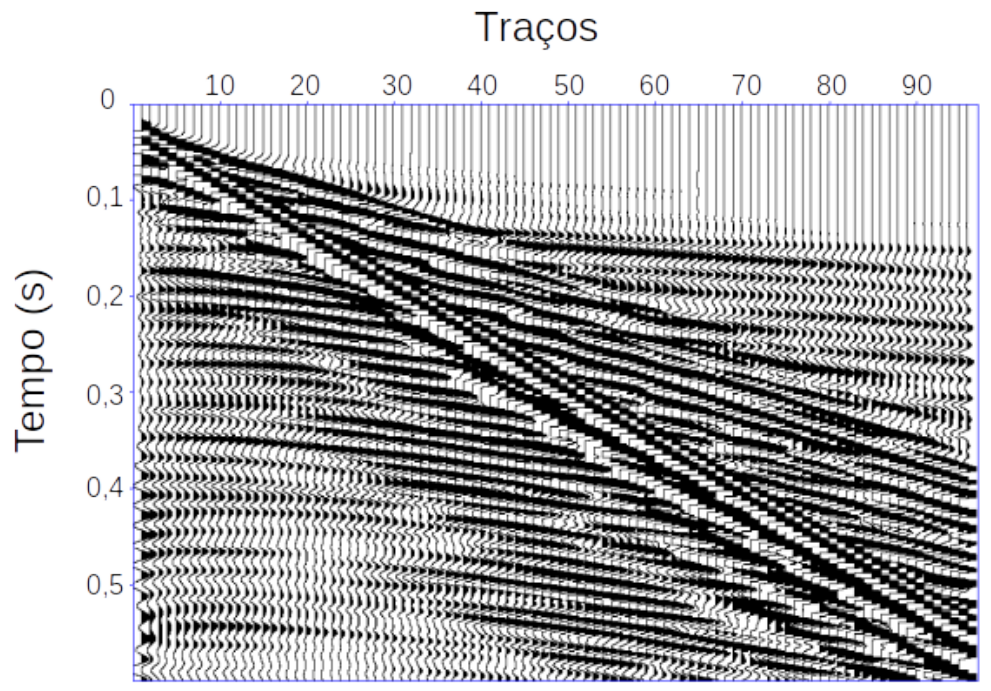

Para a análise de sensibilidade da função objetivo em relação a cada um dos parâmetros do modelo, apenas o parâmetro de interesse foi perturbado e os demais parâmetros foram mantidos nos valores corretos do modelo. A variação da função objetivo em função do parâmetro adotado são apresentados nas Figuras 9 e 10. 
Figura 9 - Análise de sensibilidade para a janela de 1 a 48 metros. Valor da função objetivo pela variação do parâmetro buscado.
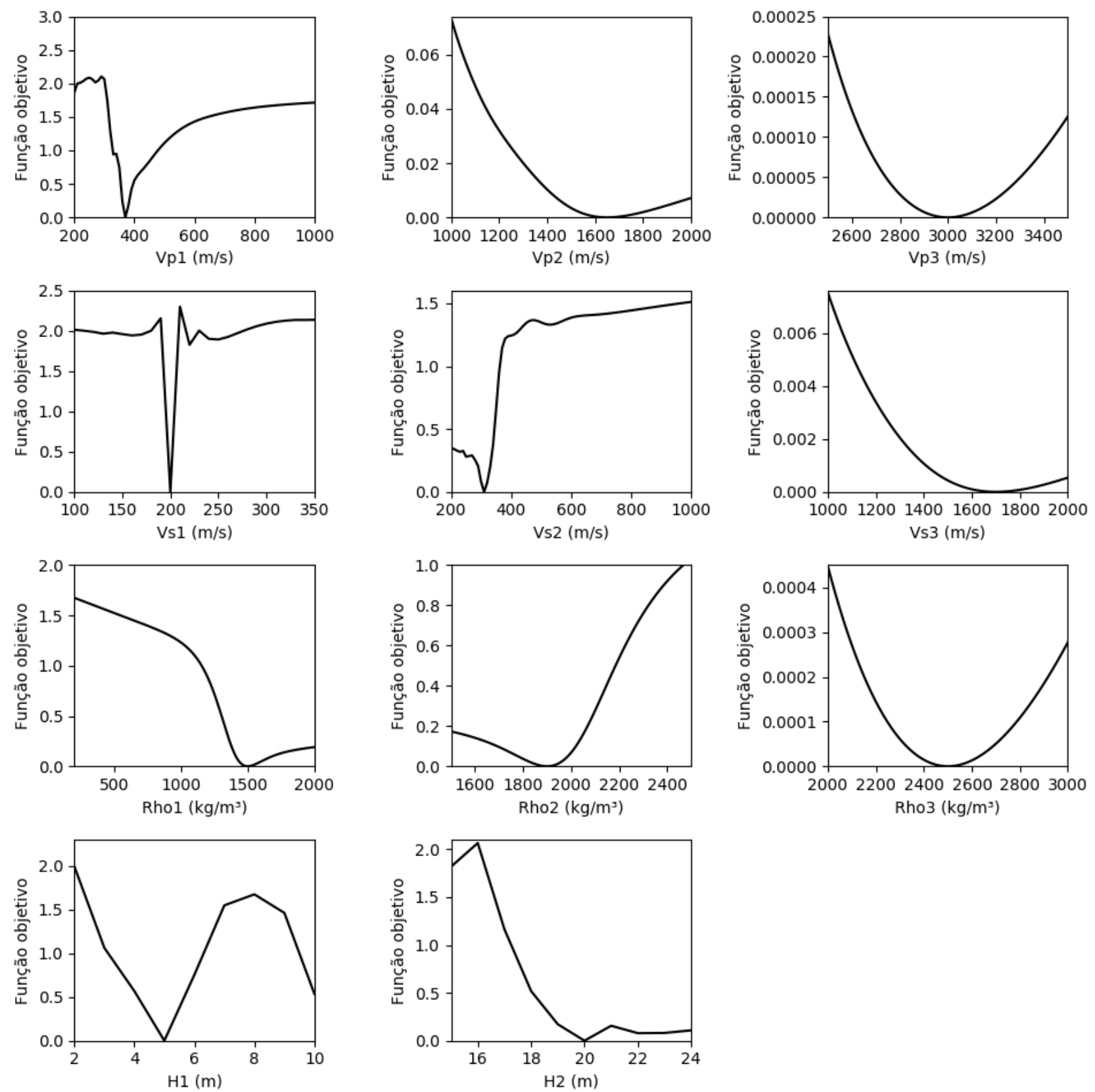

Comparando as Figuras 9 e 10 é possível notar que os padrões de variação da função objetivo em relação aos parâmetros apresentam semelhanças considerando-se as duas janelas de afastamentos. Sendo que os parâmetros do semiespaço $\left(V_{P 3}, V_{S 3}\right.$ e $\left.\rho_{3}\right)$ são os que apresentam menor influência na função objetivo (notar escala de valores dos gráficos). As velocidades da onda $\mathrm{P}$ na primeira camada e a da onda $\mathrm{S}$ na primeira e segunda camadas $\left(V_{P 1}, V_{S 1}\right.$ e $\left.V_{S 2}\right)$ geram um gradiente elevado na Fobj em relação aos demais parâmetros. As densidades $\left(\rho_{1}\right.$ e $\left.\rho_{2}\right)$ apresentam menor sensibilidade para a janela 
Figura 10 - Análise de sensibilidade para a janela de 49 a 96 metros. Valor da função objetivo pela variação do parâmetro buscado.
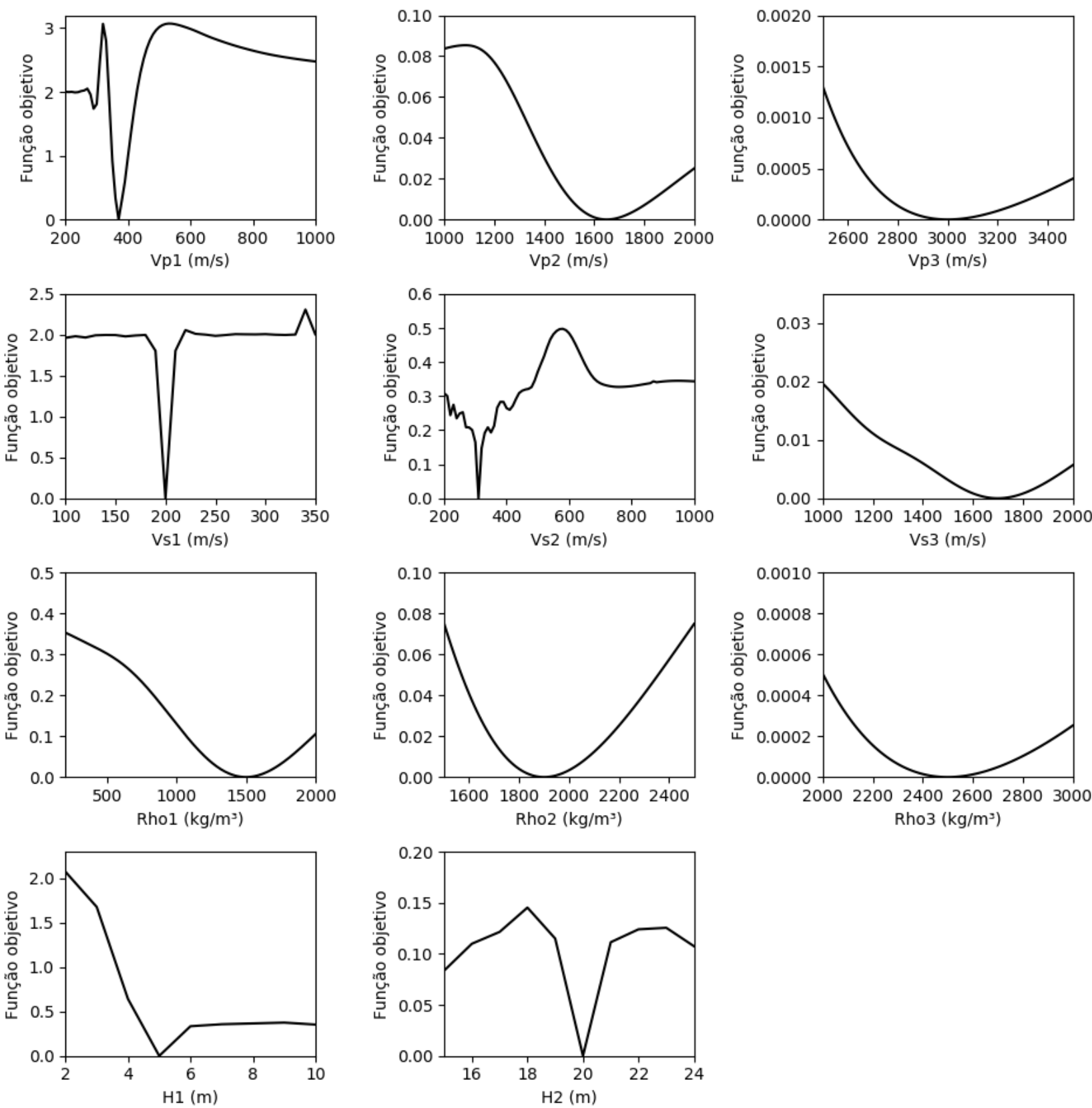

apenas com afastamentos longos (Figura 10), já os resultados empregando também os afastamentos curtos (Figura 9) indicam que as densidades poderiam ser estimadas. Quanto as profundidades, as análises mostram baixa sensibilidade apenas na janela que contém os afastamentos curtos (Figura 9), na segunda interface para diferenciar as profundidades acima do valor real.

$\mathrm{Na}$ aplicação do algoritmo de evolução diferencial para o mapeamento da função objetivo (Figuras 11 e 12) foram obtidas conclusões semelhantes as do teste de sensibilidade 
de parâmetros. Os parâmetros nas camadas superiores e a velocidade da onda S em todas as camadas foram melhor estimados. A convergência para os valores corretos foram melhores para a janela de menor afastamento (Figura 11). Para a janela de 49 a 96 metros (Figura 12) o parâmetro $V_{S}$ e a profundidade das interfaces são os que mais se aproximam do valor real. Porém, com um erro maior quando comparados a estimativa da janela de menor afastamento.

O comportamento da função objetivo deve ser analisado com cautela. Horn (1993) explica que algoritmos genéticos sofrem de pressão de seleção e deriva genética e segundo Aleardi e Mazzotti (2017) isto limita a exploração do espaço do modelo podendo guiar o algoritmo de forma prematura para um mínimo local. Porém, acredita-se que a aglomeração de pontos em torno da função objetivo seja um indicativo da sensibilidade da Fobj para cada parâmetro.

Para a utilização do algoritmo de evolução diferencial foi escolhida uma população de 1100, um número de iterações igual a 10, um fator de mutação 0,09 e de recombinação de 0,8 . Esta configuração foi utilizada para as duas janelas de afastamento. 
Figura 11 - Função objetivo pelos parâmetros estimados. Pontos testados durante a aplicação do algoritmo de evolução diferencial. Janela de 1 a 48 metros.
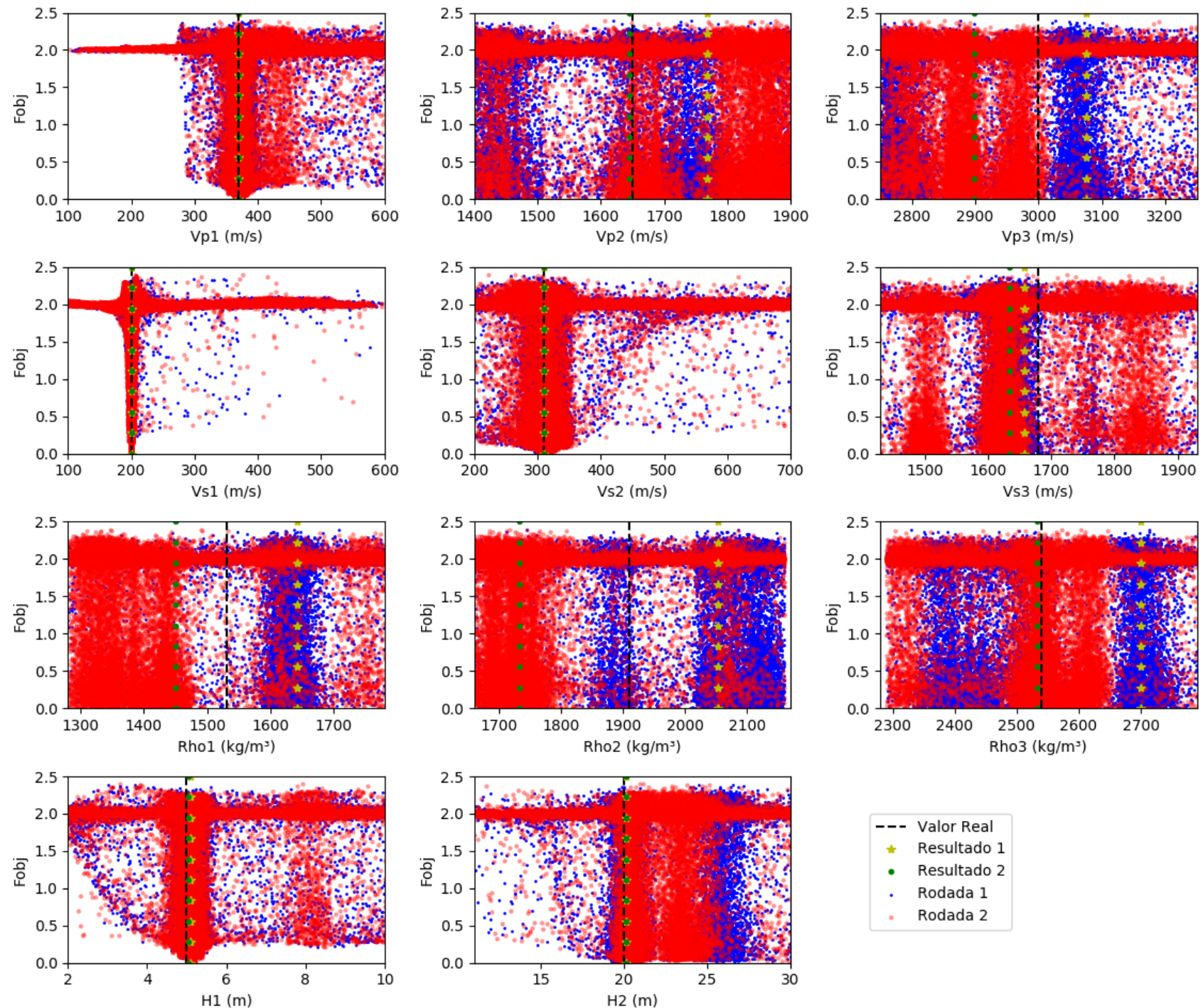
Figura 12 - Função objetivo pelos parâmetros estimados. Pontos testados durante a aplicação do algoritmo de evolução diferencial. Janela de 49 a 96 metros.
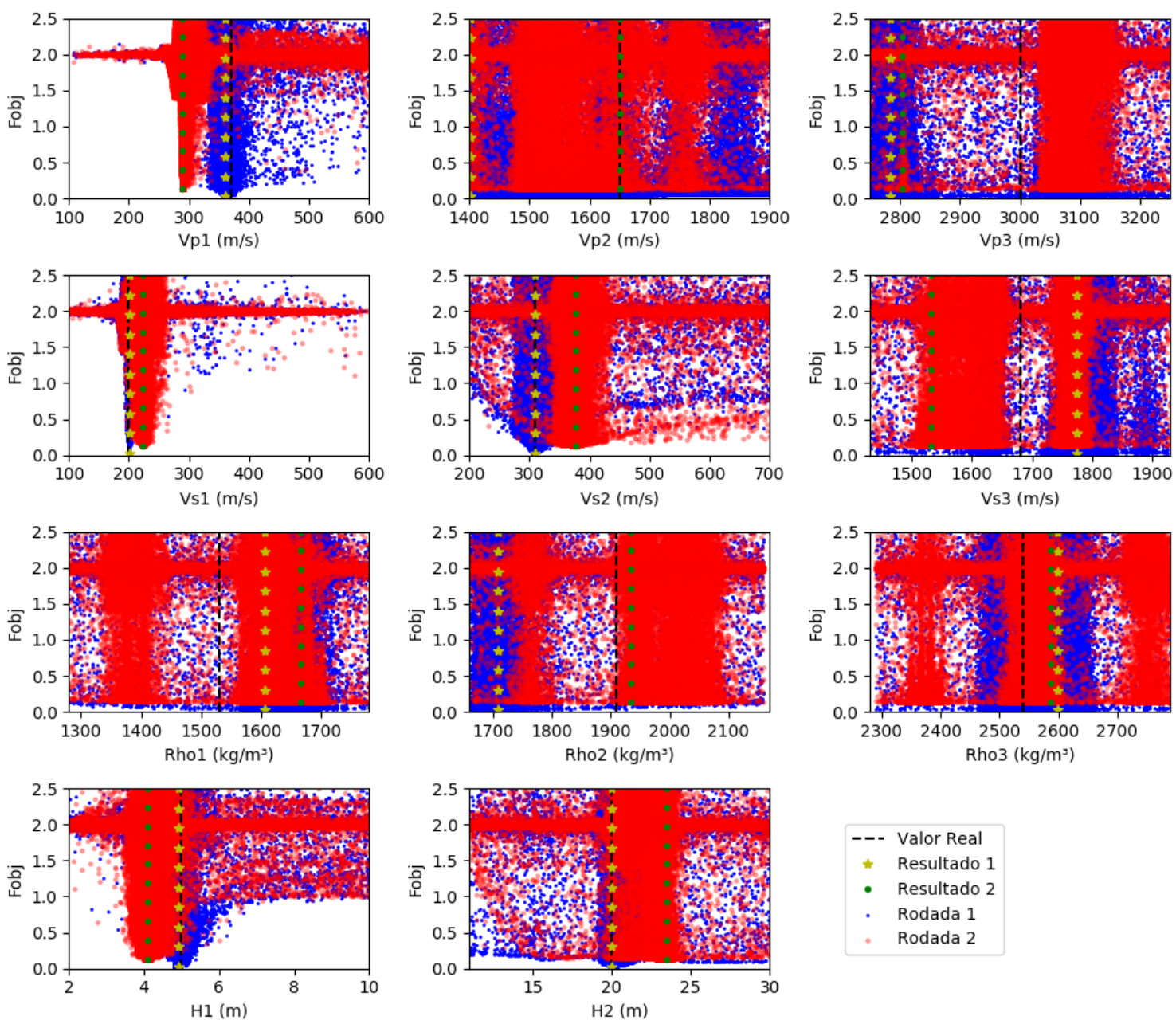


\subsection{Testes com a estratégia local: algoritmo de inversão tomográ- fica}

\subsubsection{Análise da estimativa individual dos parâmetros}

Em Groos et al. (2017) é mencionado que algoritmos de tomografia possuem problemas de convergência na presença de mudanças drásticas dos valores dos parâmetros e que as interfaces definidas no modelo inicial raramente mudam de posição, ou seja, a solução final é fortemente dependente da informação a priori. Outro ponto é a dificuldade da estimativa concomitante de parâmetros. Por isso, nos primeiros testes foram simulados modelos reais onde apenas os parâmetros das camadas variam quando comparados os modelos iniciais e reais. Neste caso a profundidade das interfaces foi mantida constante. Também foram testadas situações em que os parâmetros dos modelos crescem linearmente com a profundidade.

No primeiro teste foi estimada a velocidade da onda S, que é o parâmetro mais facilmente determinado conforme os testes anteriores. Os demais parâmetros dos modelos foram assumidos como conhecidos. Na Figura 13 são apresentados os modelos real e inicial (notar escala de valores na legenda de cores). Neste caso foi simulado um ponto de tiro na posição 5 metros e 48 geofones equidistantes e dispostos no intervalo de 53 a 100 metros, conforme indicado na imagem do modelo real da Figura 13. A Figura 14 traz o resultado final que apresenta variação lateral de velocidade ao no do modelo. Para facilitar a comparação entre os modelos foi construída a Figura 15 que mostra o valor das estimativas de forma mais detalhada em diferentes posições do modelo.

Nos primeiros metros de profundidade do modelo a variação lateral é maior. O resultado final mostra relação com a posição do ponto de tiro e com a posição do arranjo de geofones, além do caminho percorrido pela onda: a área mais "iluminada"pela propagação das ondas apresenta o melhor resultado. Assim o problema de estimativa de velocidade da onda S foi estendido para uma situação com mais tiros.

No próximo teste foi avaliada uma situação no mesmo modelo com três tiros posicionados conforme o modelo real mostrado na Figura 13, nas posições (5, 16 e 28 metros). Este resultado foi semelhante ao caso com um único tiro e portanto foi realizado outro teste com pelo menos um ponto de tiro mais próximo dos receptores, posições (5, 28 e 52 metros), cujos resultados se mostraram satisfatórios (Figuras 16 e 17). Sendo que apenas neste último teste foram obtidos resultados satisfatórios. 
Figura 13 - Modelos real e inicial para a velocidade da onda S. Velocidade constante nas camadas.

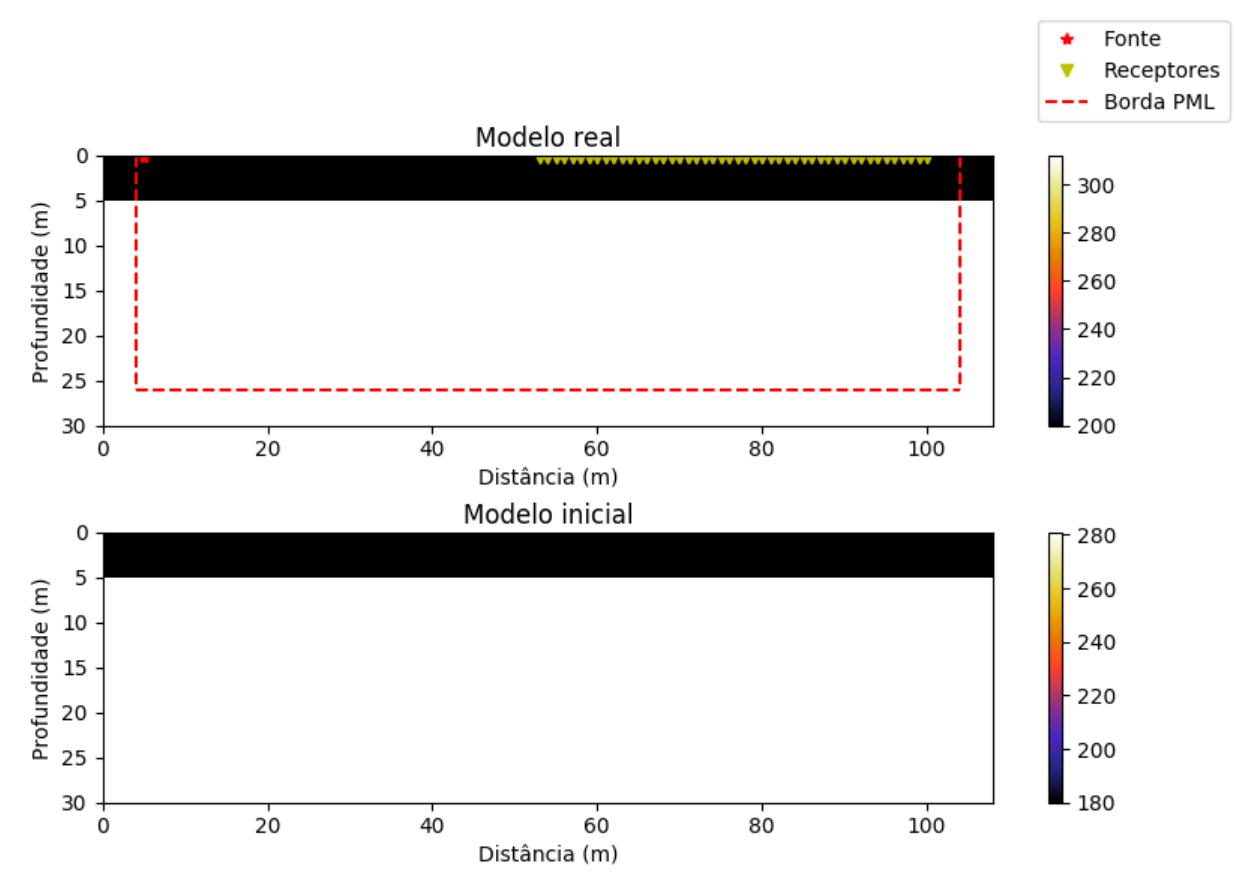

Figura 14 - Modelo de velocidade da onda S estimado no caso de um único tiro na posição de 5 metros.

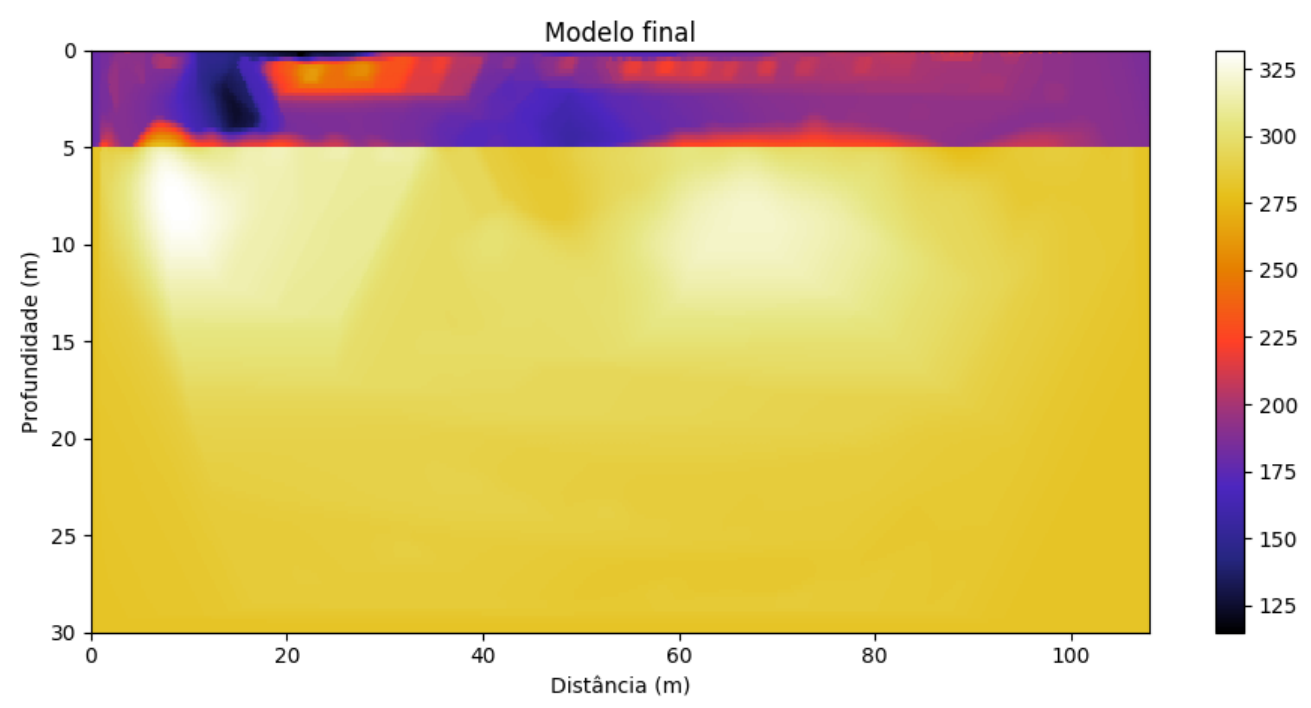


Figura 15 - Resultado em detalhe extraído em diferentes posições do modelo de velocidades da onda S da Figura 14.

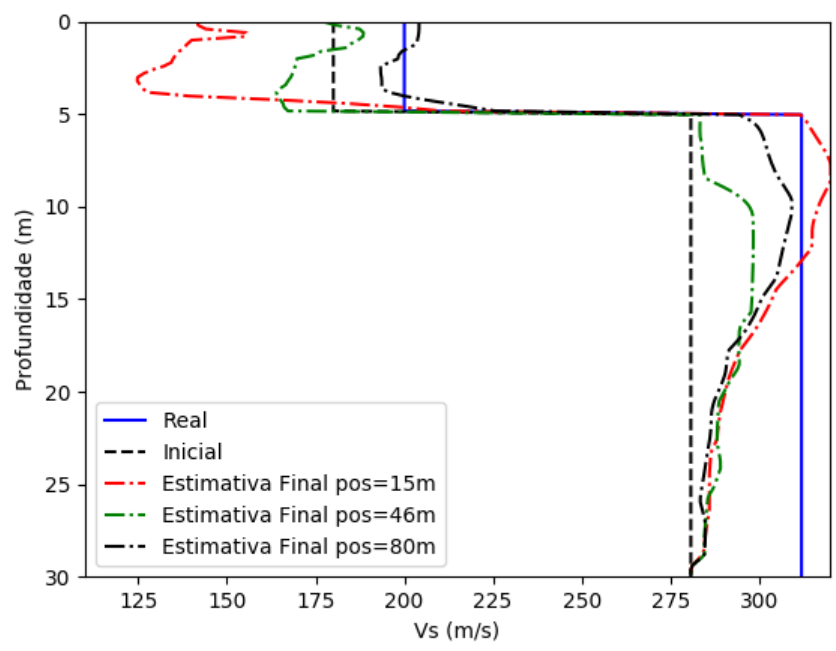

Figura 16 - Resultado para a estimativa da velocidade da onda S em uma aquisição com três pontos de tiro nas posições de 5,28 e 52 metros.

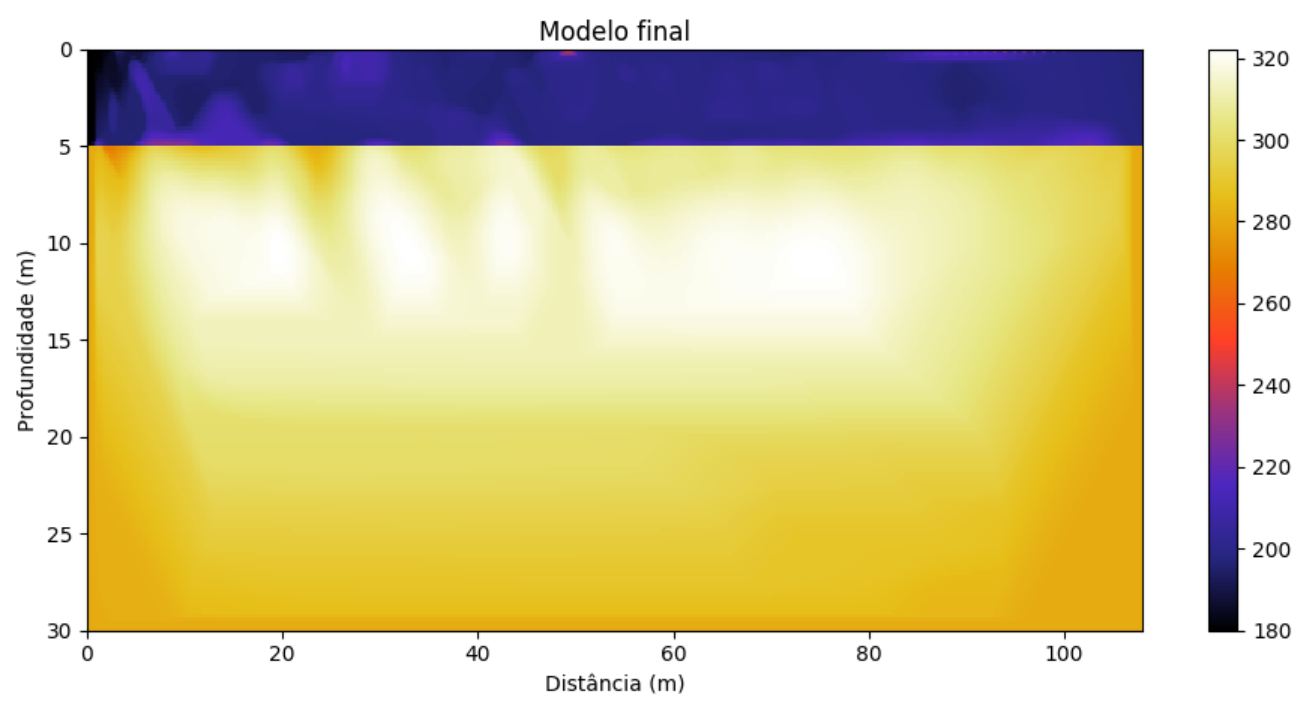


Figura 17 - Resultado em detalhe extraído em diferentes posições do modelo de velocidades da onda S da Figura 16.

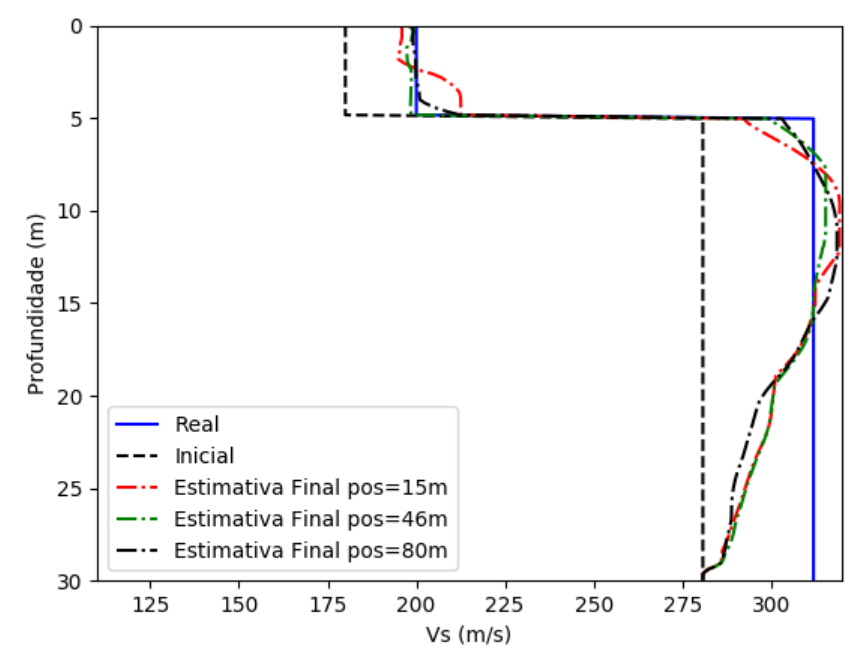

No passo seguinte foi testado o modelo com crescimento linear dos parâmetros com a profundidade. A partir deste teste todos os resultados foram obtidos com três pontos de tiro nas posições 5, 28 e 52 metros, da mesma forma que o teste anterior. A diferença entre o modelo real e o inicial é o valor no topo do modelo e o gradiente-vertical dos parâmetros. Da mesma forma que o teste anterior apenas a velocidade da onda $\mathrm{S}$ foi estimada mantendo os demais parâmetros nos valores corretos. Neste teste ainda é observada dificuldade na convergência para o modelo real (Figuras 18 e 19). Neste caso ainda são notadas regiões no modelo relacionadas aos pontos de tiro. Como analisado no caso anterior, este tipo de problema pode ser tratado aumentando a cobertura de pontos de tiro no modelo. Porém, outras formas de controlar este efeito foram analisadas nos tópicos posteriores. 
Figura 18 - Modelos real, inicial e final para a inversão da velocidade da onda $\mathrm{S}$ em modelos com crescimento linear do parâmetro com a profundidade.
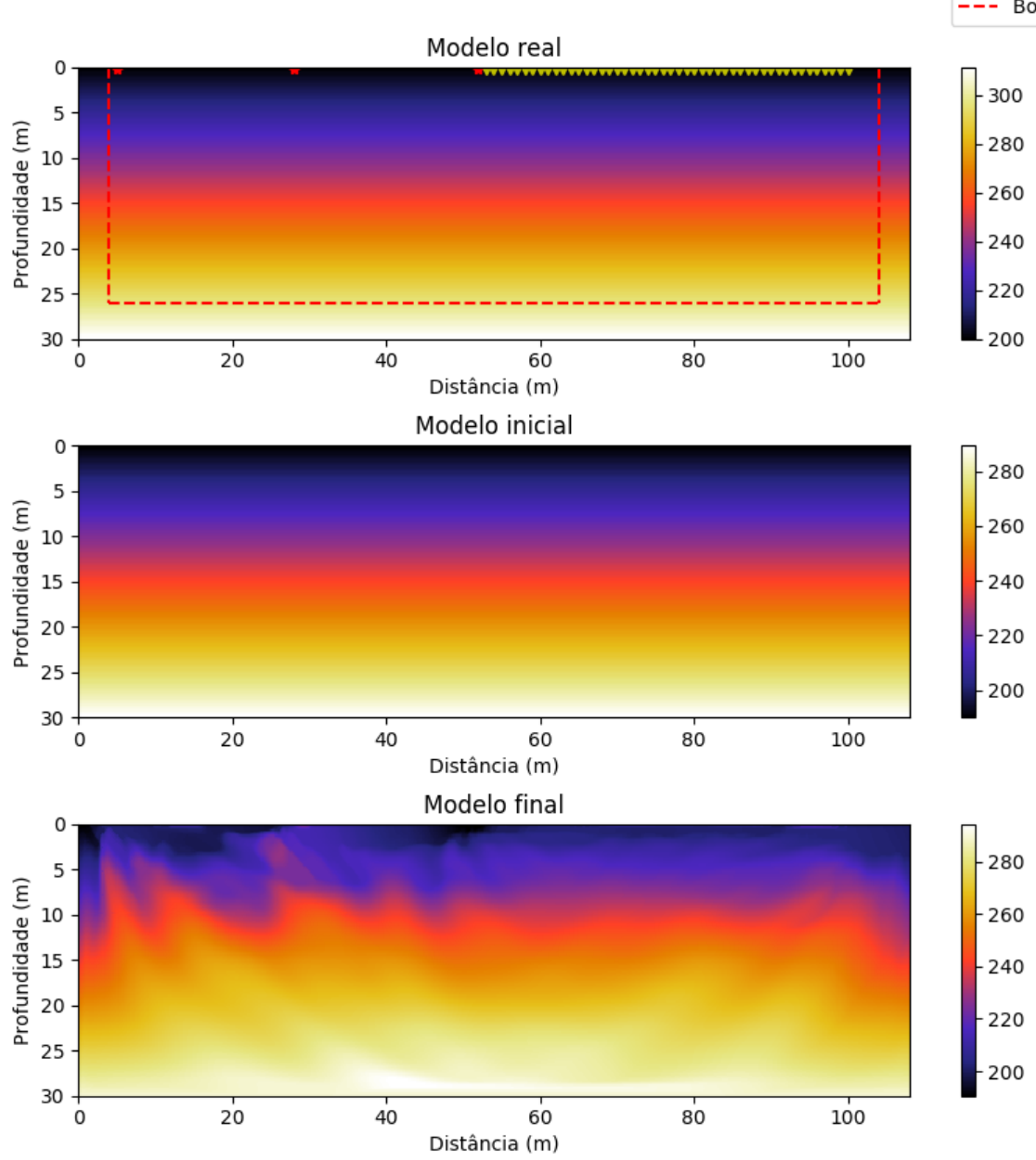

Figura 19 - Resultado em detalhe extraído em diferentes posições do modelo de velocidades da onda S da Figura 18.

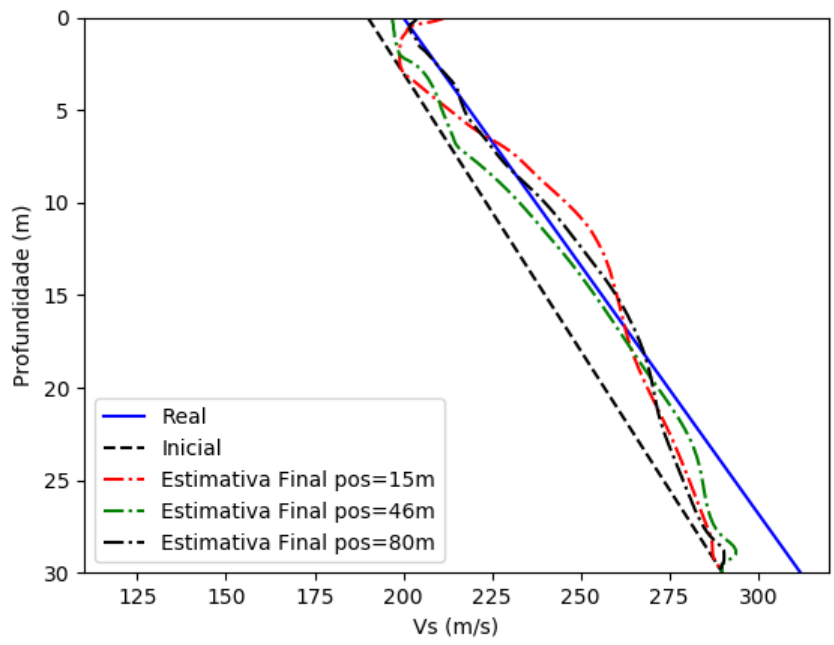


O mesmo tipo de teste foi reproduzido para os parâmetros $V_{P}$ e $\rho$. No caso velocidade da onda P (Figuras 20 e 21) foi percebida pouca modificação do valor do parâmetro.

Figura 20 - Resultado para a estimativa da velocidade da onda P no modelo de camadas em uma aquisição com três pontos de tiro nas posições de 5,28 e 52 metros.

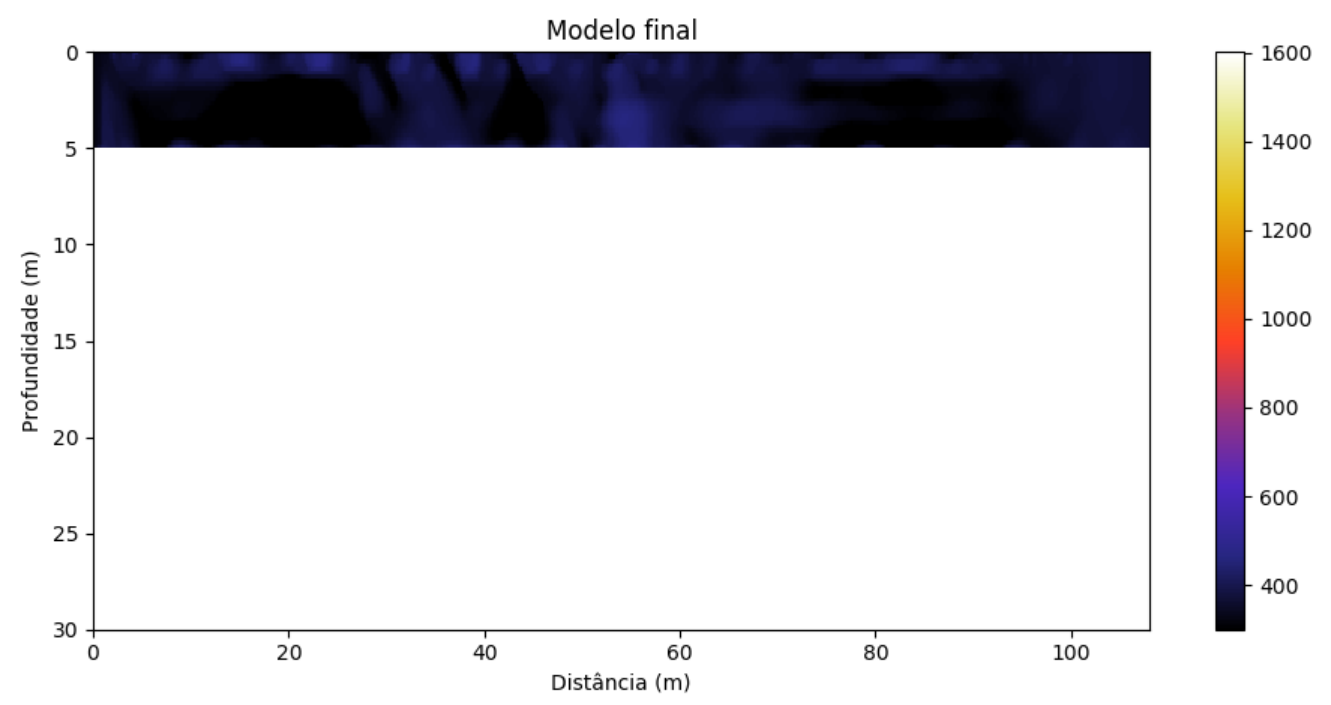

Figura 21 - Resultado em detalhe extraído em diferentes posições do modelo de velocidades da onda S da Figura 20.

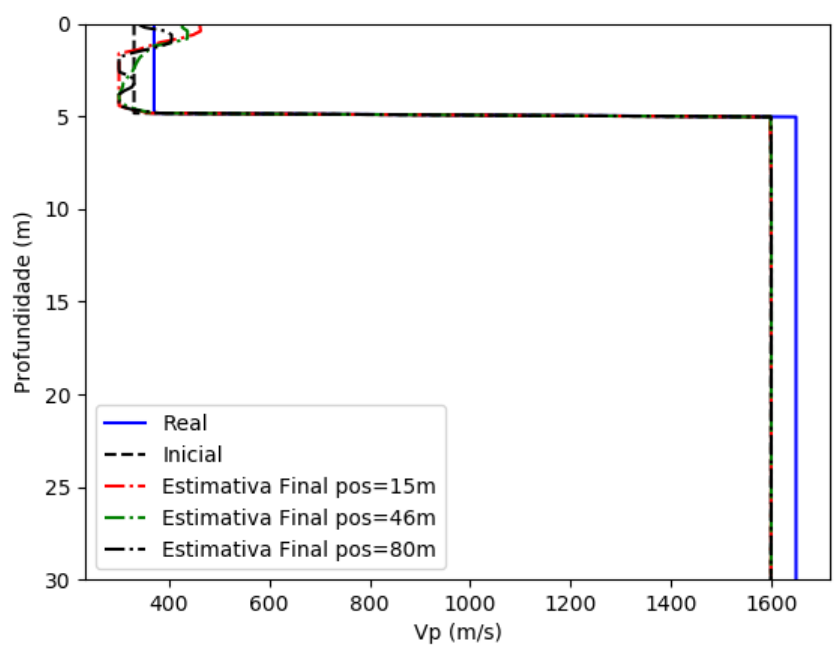

Como os resultados mostrado nas Figuras 20 e 21 não foram satisfatórios foi realizado mais um teste com um modelo inicial mais próximo do modelo real (Figura 22). Na sequência foi avaliado o modelo com crescimento linear na $V_{P}$ (Figuras 23 e 24 e realizados os testes para o parâmetro de densidade: constante na camada (Figuras 25 e 26) e com variação linear com a profundidade (Figuras 27 e 28). 
Figura 22 - Resultado em detalhe extraído em diferentes posições do modelo de velocidades da onda $\mathrm{P}$ em um caso com uma diferença menor entre o modelo inicial e real.

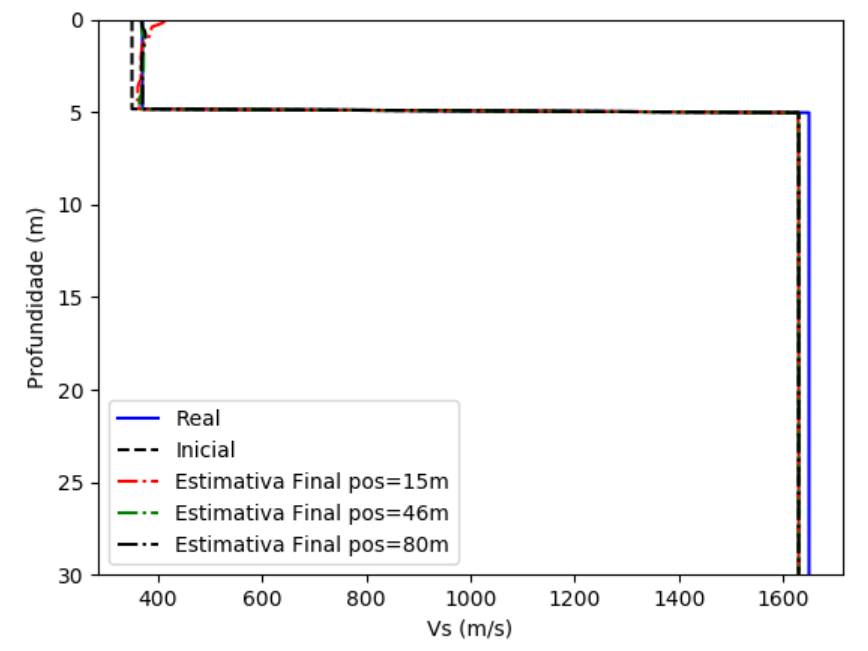

Figura 23 - Resultado para a estimativa da velocidade da onda P no modelo com crescimento linear do parâmetro em uma aquisição com três pontos de tiro nas posições de 5,28 e 52 metros.

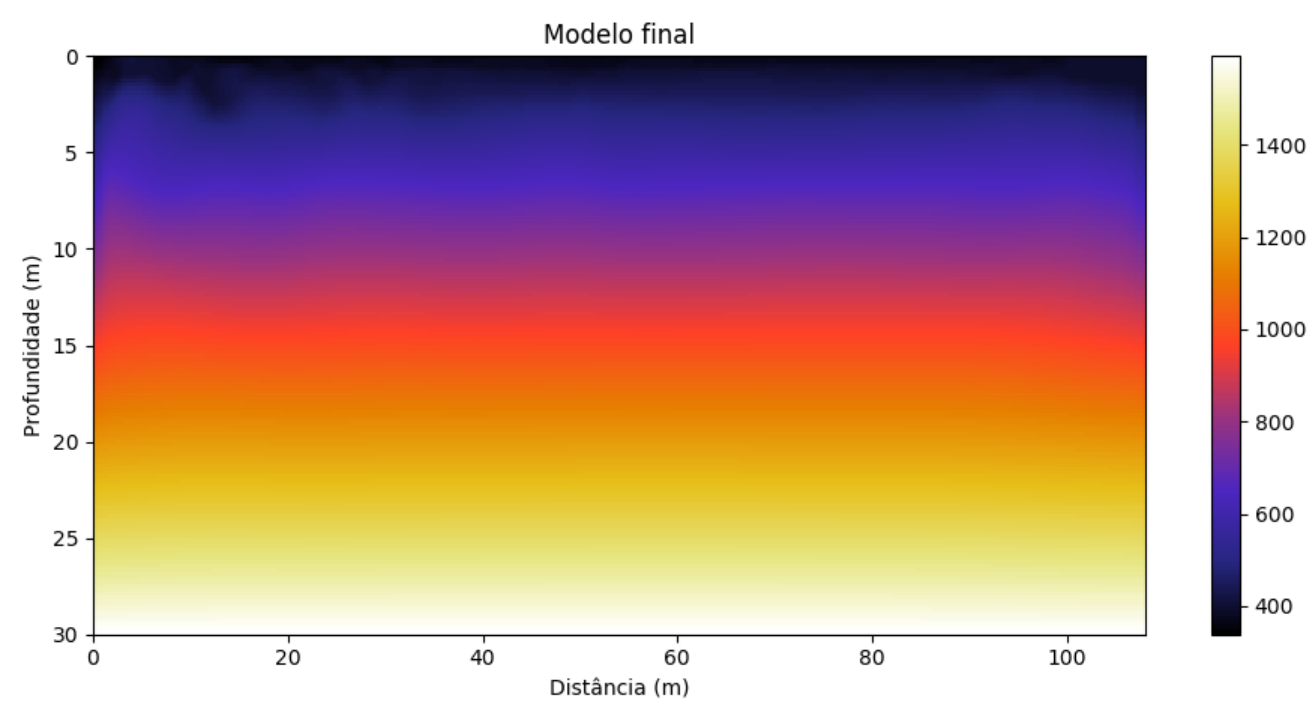


Figura 24 - Resultado em detalhe extraído em diferentes posições do modelo de velocidades da onda S da Figura 23.

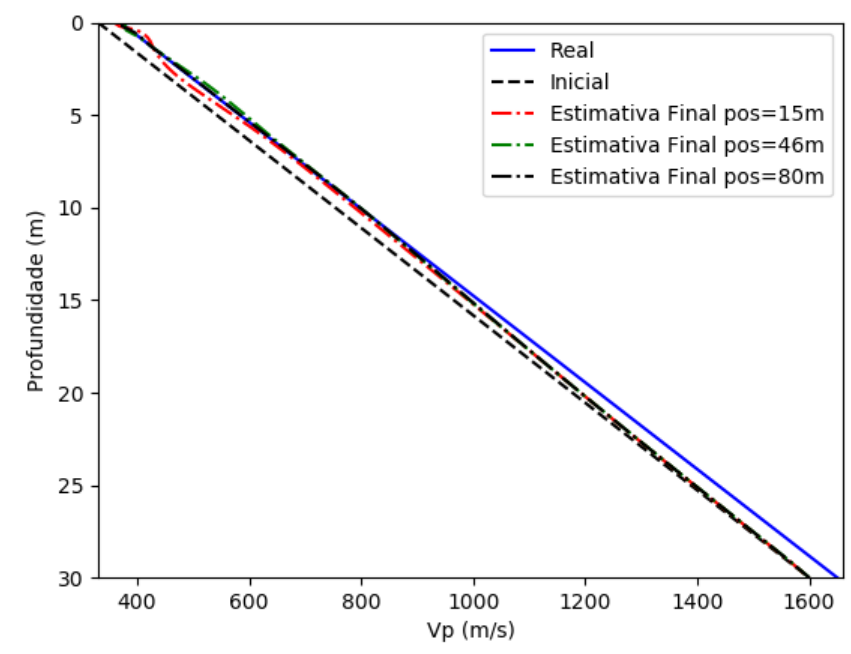

Figura 25 - Resultado para a estimativa da densidade no modelo de camadas em uma aquisição com três pontos de tiro nas posições de 5,28 e 52 metros.

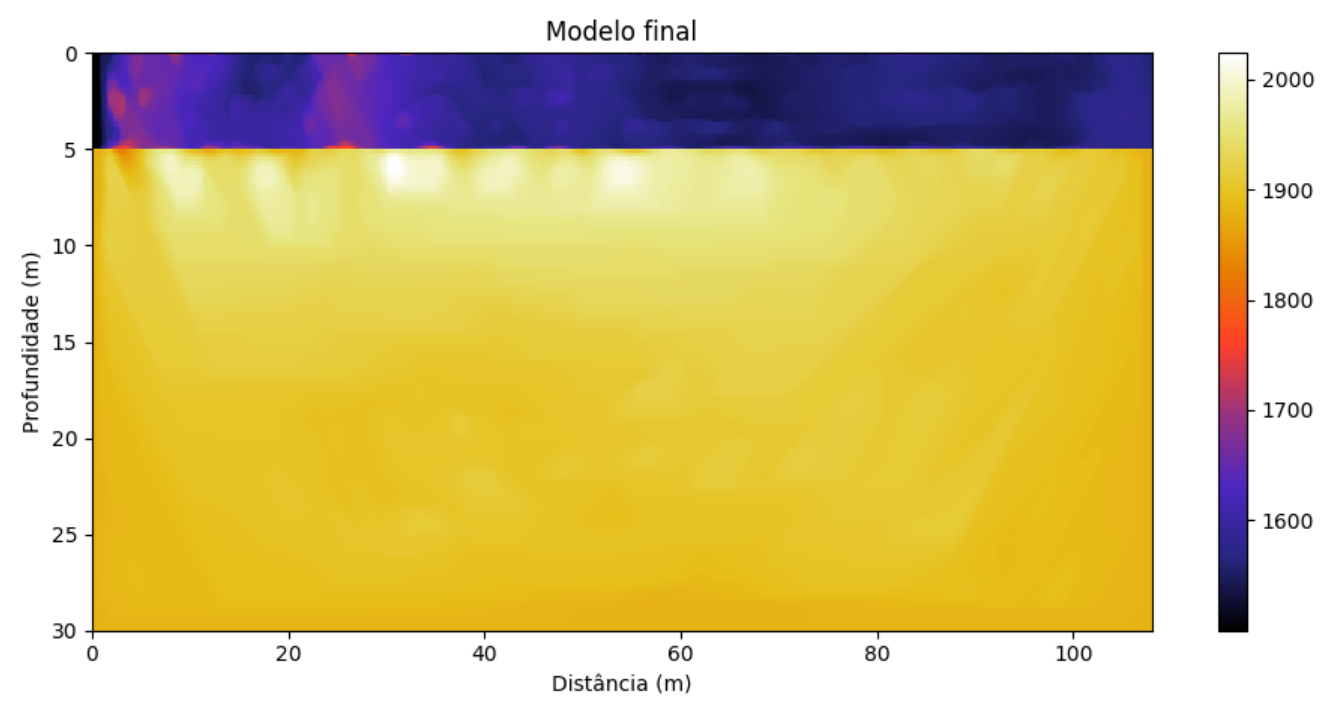


Figura 26 - Resultado em detalhe extraído em diferentes posições do modelo de densidades da Figura 25.

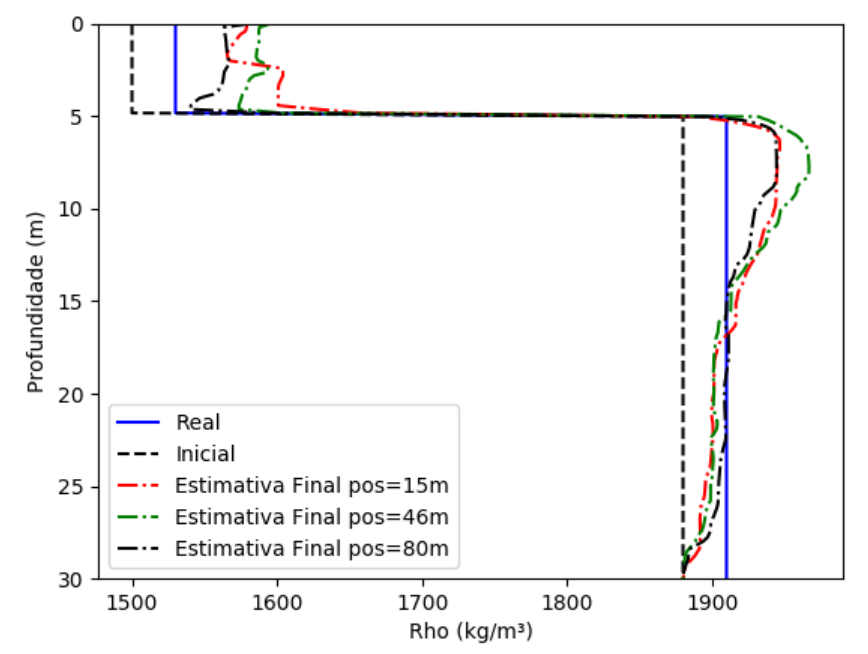

Figura 27 - Resultado para a estimativa da densidade no modelo com crescimento linear do parâmetro em uma aquisição com três pontos de tiro nas posições de 5,28 e 52 metros.

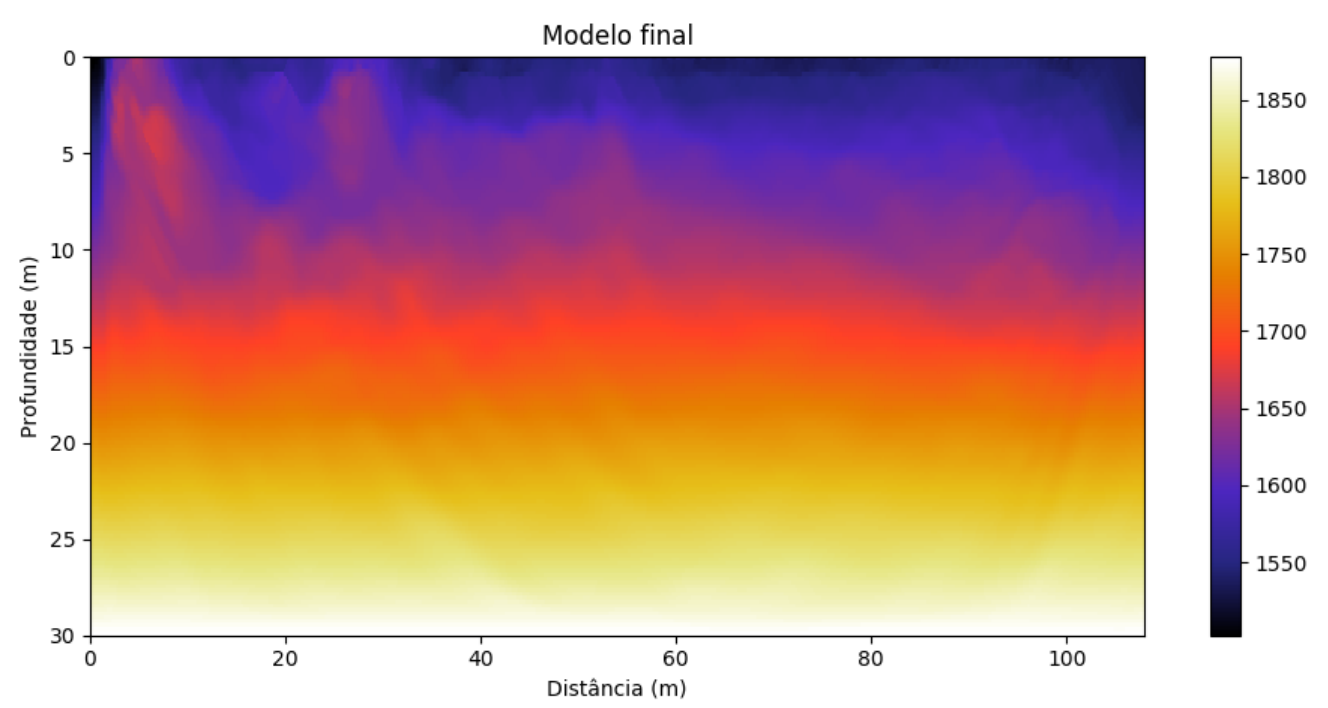


Figura 28 - Resultado em detalhe extraído em diferentes posições do modelo de densidades da Figura 27.

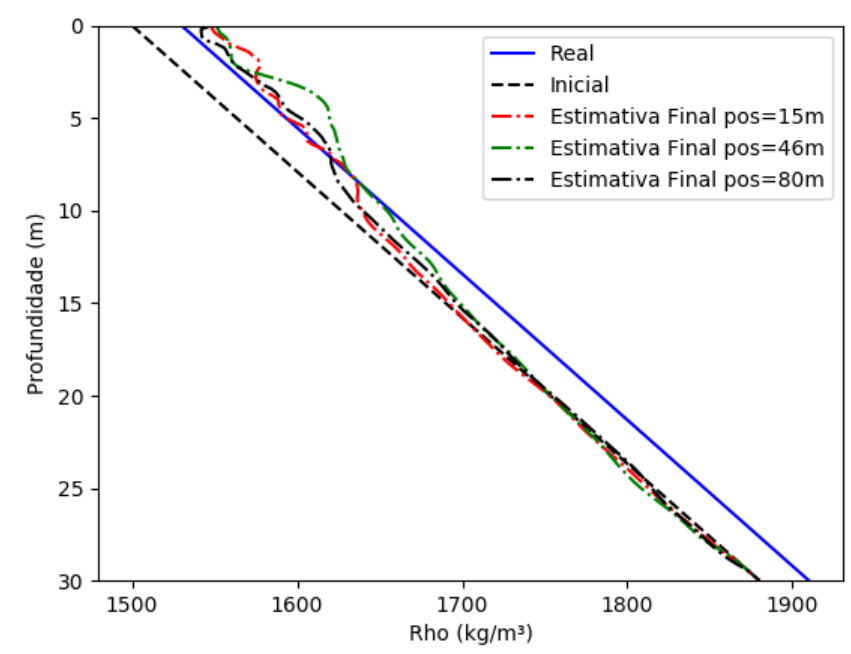

As respostas obtidas para $V_{P}$ deixam clara a necessidade da proximidade que o modelo inicial deve ter em relação ao modelo real. Apesar dos valores iniciais de $V_{P}$ serem próximos do real, ocorre um refinamento da $V_{P}$ na parte mais rasa do modelo, como mostrado nas Figuras 22 e 24. A estimativa da densidade tende ao modelo real porém com uma resposta um pouco instável. O programa tem uma opção de suavizar os modelos $V_{P}$ e $V_{S}$, não existe para o modelo de densidade.

\subsubsection{Estimativa concomitante de parâmetros}

Os mesmos modelos do tópico anterior foram utilizados na estimativa concomitante de parâmetros. Primeiro foi realiada a estimativa conjunta dos parâmetros $V_{P}$ e $V_{S}$ e posteriormente foi avaliada a estimativa dos três parâmetros $V_{P}, V_{S}$ e $\rho$ em conjunto. Assim como em (SPADINI et al., 2016) foi realizada uma comparação entre a estimativa padrão que está no código IFOS2D com a estimativa onde apenas a velocidade da onda $\mathrm{S}$ é atualizada pelo algoritmo de gradiente conjugado e os parâmetros $V_{P}$ e $\rho$ são atualizados pelas relações mostradas nas equações 3.8 e 3.9 .

No caso dos modelos com parâmetros constantes nas camadas foi utilizado o modelo $V_{P}$ inicial mais próximo do modelo real, da mesma forma que no resultado mostrado na Figura 22. Entretanto, o resultado final para o modelo $V_{P}$ não convergiu para a resposta desejada. Já o modelo de velocidades da onda $\mathrm{S}$ se aproximou de forma satisfatória para o modelo real. Apesar disso, o modelo 2D da Figura 29 apresenta variação lateral, sendo algumas regiões com velocidades abaixo e outras acima do esperado.

Já para o modelo com crescimento linear dos parâmetros foi observada convergência nos dois casos tanto $V_{P}$ quanto $V_{S}$ (Figuras 31 e 32 ). A diferença é que o parâmetro $V_{S}$ é 
Figura 29 - Resultado para a estimativa concomitante da velocidade das ondas P e S no modelo de camadas em uma aquisição com três pontos de tiro nas posições de 5,28 e 52 metros.
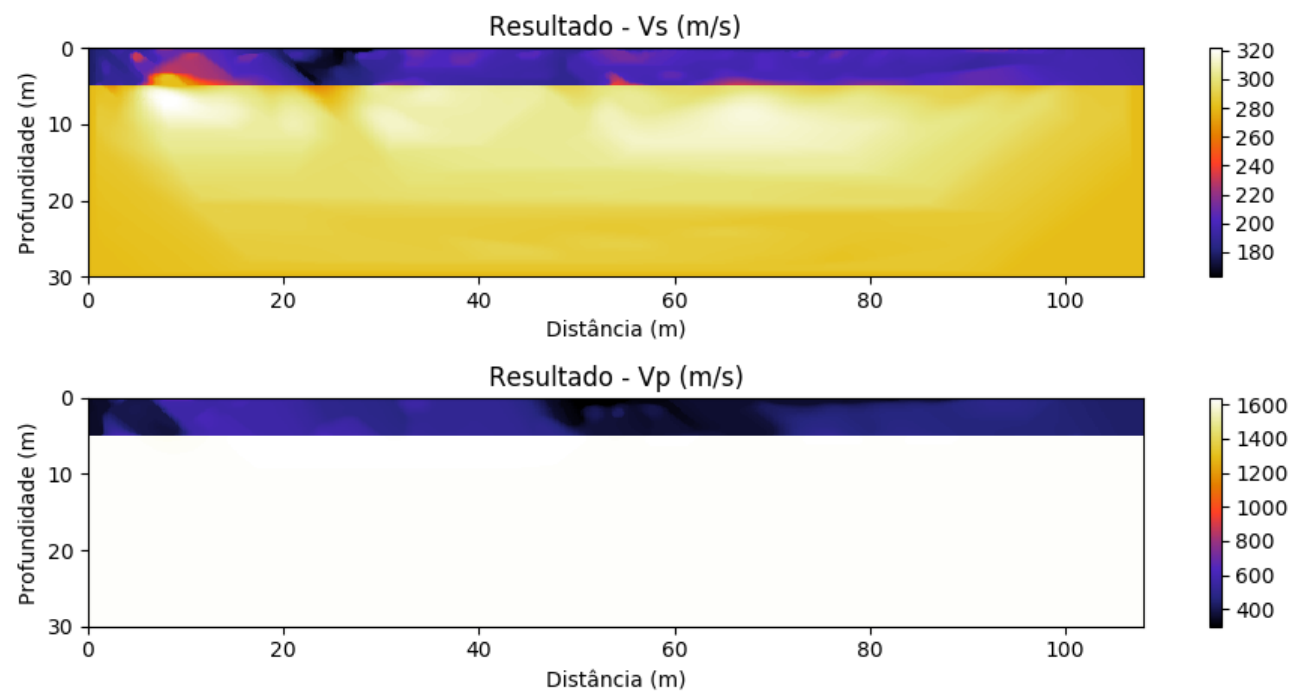

Figura 30 - Resultado em detalhe extraído em diferentes posições dos modelos da Figura 29.
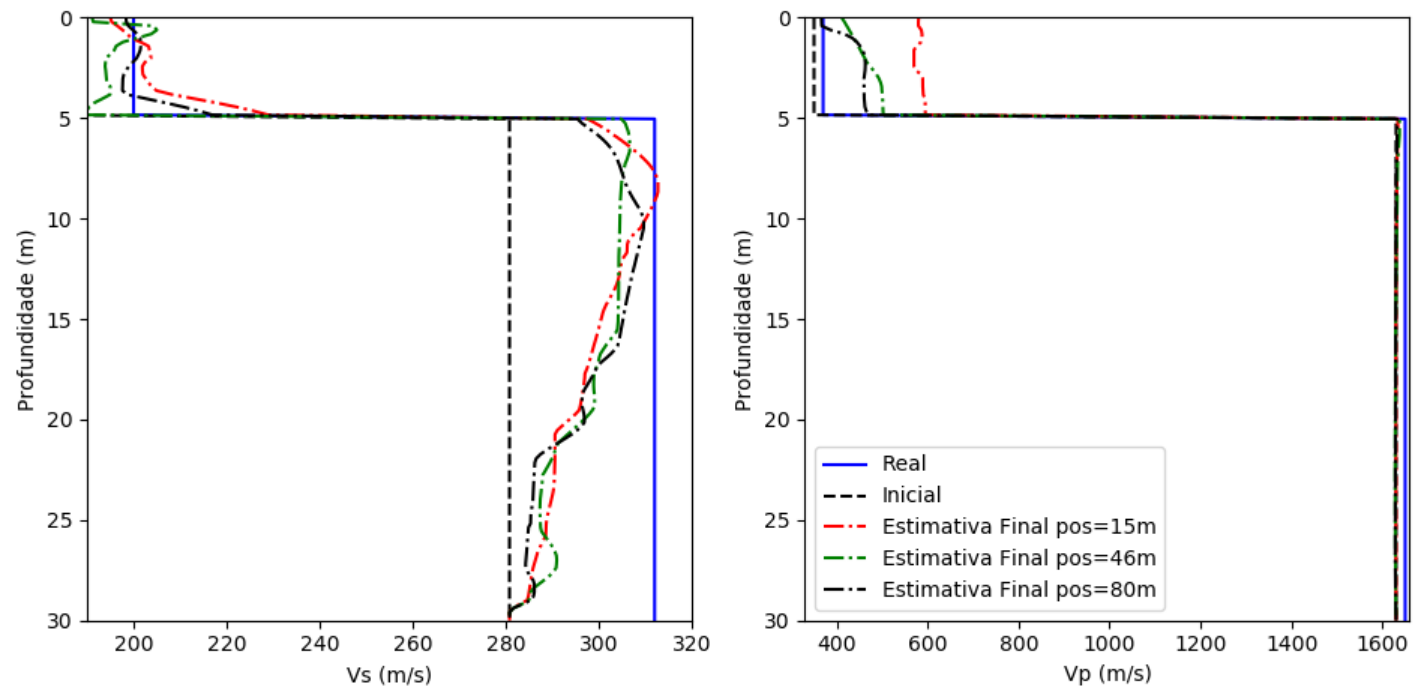

mais suscetível a variações relacionadas a cobertura de pontos de tiro. 
Figura 31 - Resultado para a estimativa concomitante da velocidade das ondas P e S no modelo com crescimento linear de parâmetros em uma aquisição com três pontos de tiro nas posições de 5,28 e 52 metros.
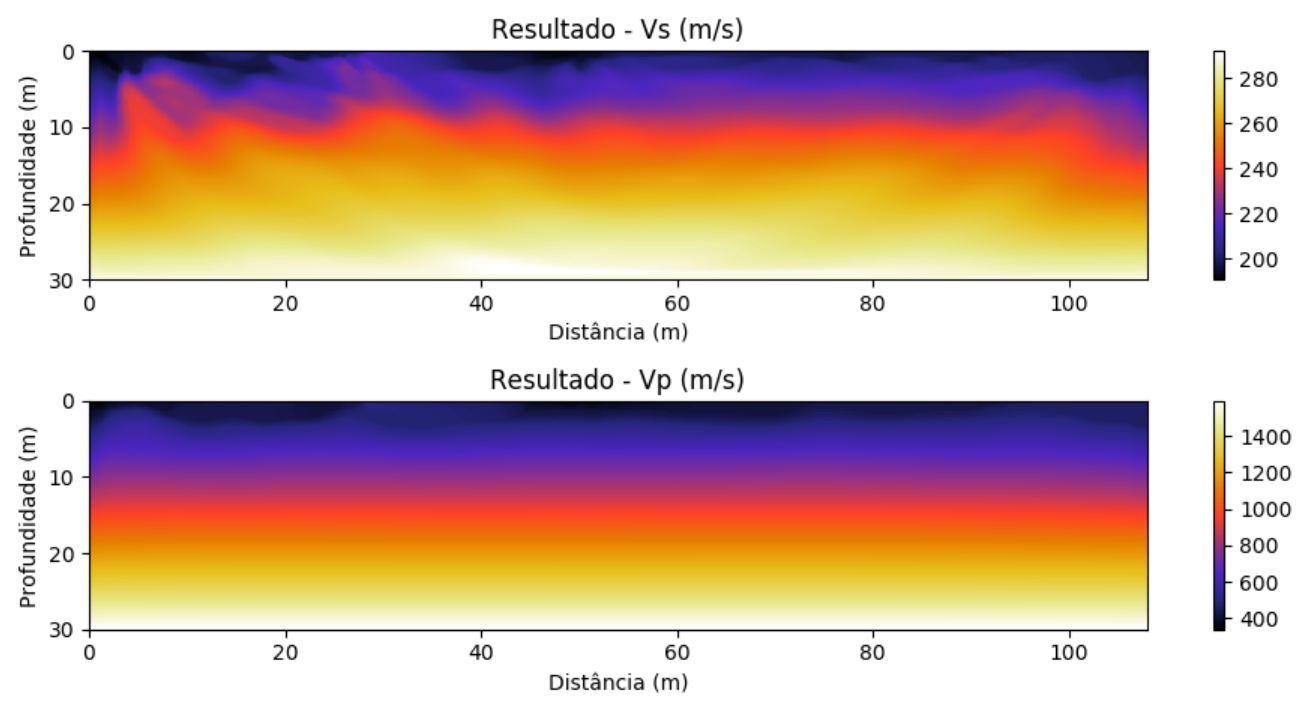

Figura 32 - Resultado em detalhe extraído em diferentes posições dos modelos da Figura 31.
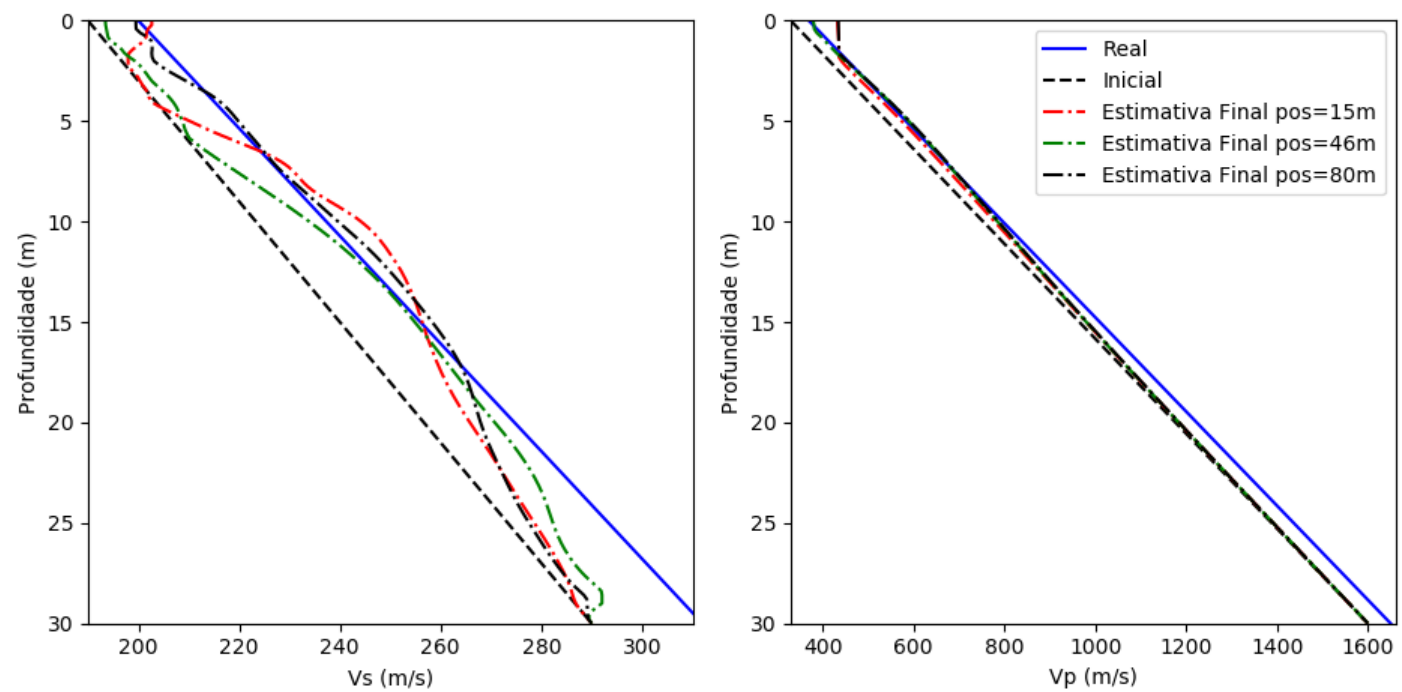

Passando para a estimativa concomitante onde uma relação entre $V_{P}$ e $V_{S}$ é utilizada foi aproveitado o algoritmo de evolução diferencial para a determinação dos parâmetros $\alpha$, $\beta$ e $\gamma$ (Equações 3.8 e 3.9). A relação foi obtida a partir dos modelos reais. Por se tratar de um modelo simples o ajuste foi perfeito.

Os resultados obtidos utilizando uma relação entre $V_{P}$ e $V_{S}$ sofrem com a grande variabilidade na estimativa da velocidade da onda $S$. Este efeito é causado pela cobertura de pontos de tiro no modelo e também por uma regularização inadequada. Entretanto, os modelos $V_{P}$ obtidos apresentam velocidades dentro de um intervalo adequado para a 
Figura 33 - Resultado para a estimativa concomitante da velocidade das ondas P e S no modelo de camadas, onde Vp é estimada através de uma relação com Vs, em uma aquisição com três pontos de tiro nas posições de 5, 28 e 52 metros.
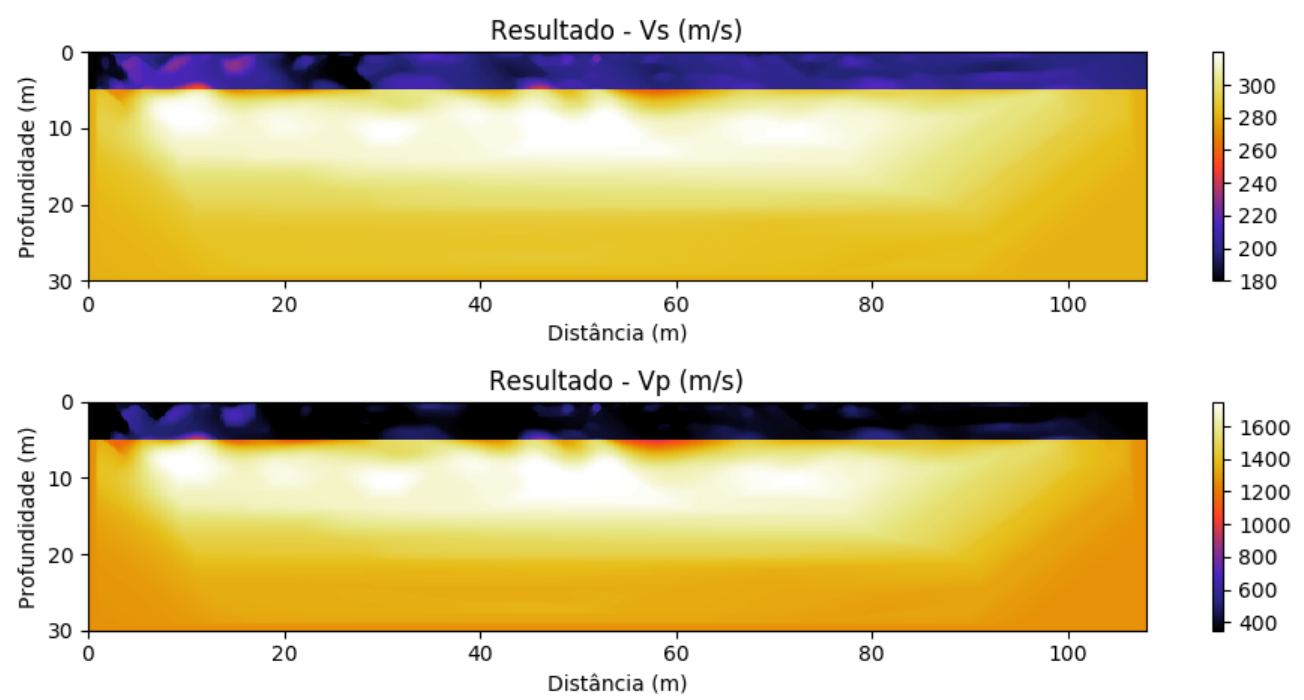

Figura 34 - Resultado em detalhe extraído em diferentes posições dos modelos da Figura 33.
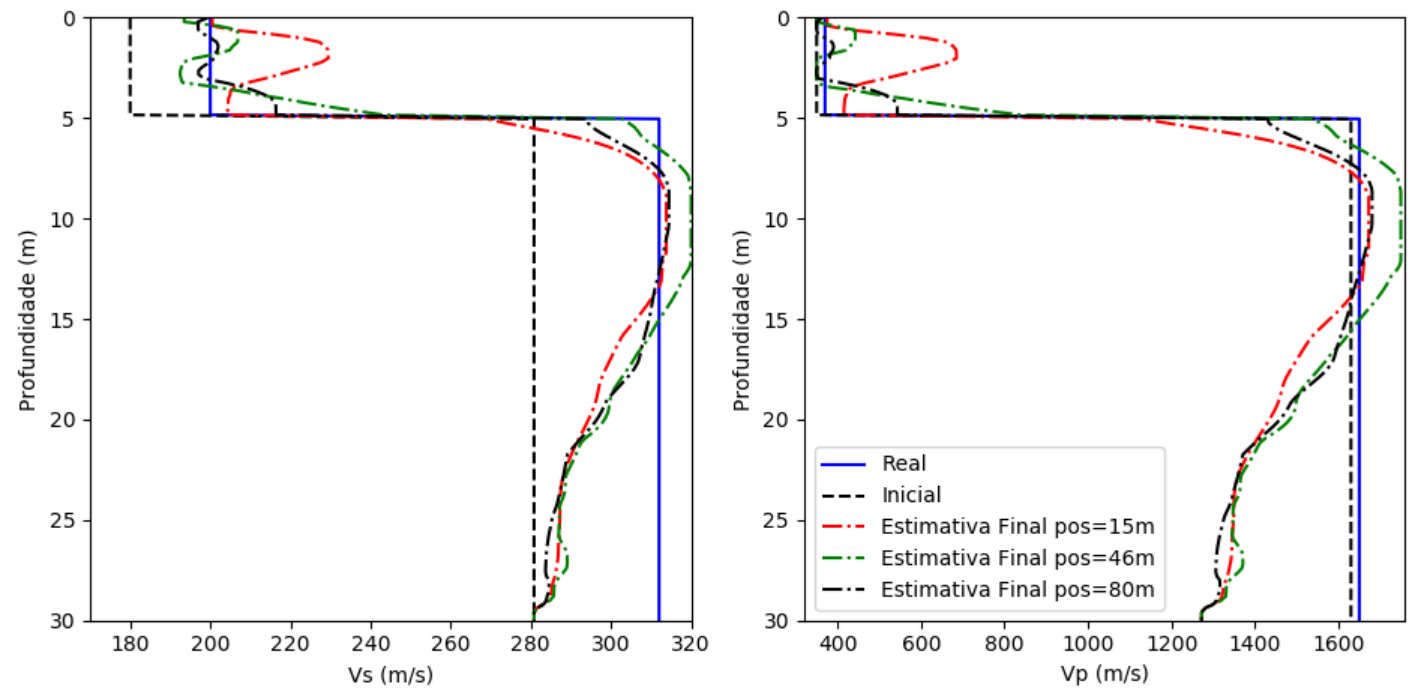

velocidade da onda P. Já o parâmetro $V_{S}$ apresenta convergência semelhante a observada quando a estimativa concomitante de parâmetros utiliza o gradiente conjugado para os dois parâmetros (Figuras 32 e 36).

Seguindo para a estimativa conjunta dos parâmetros $V_{P}, V_{S}$ e $\rho$, as imagens geradas mostram mais heterogeneidades nos modelos finais obtidos (Figuras 37 e 39). 
Figura 35 - Resultado para a estimativa concomitante da velocidade das ondas $\mathrm{P}$ e $\mathrm{S}$ no modelo com crescimento linear de parâmetros e relação Vp e Vs em uma aquisição com três pontos de tiro nas posições de 5,28 e 52 metros.
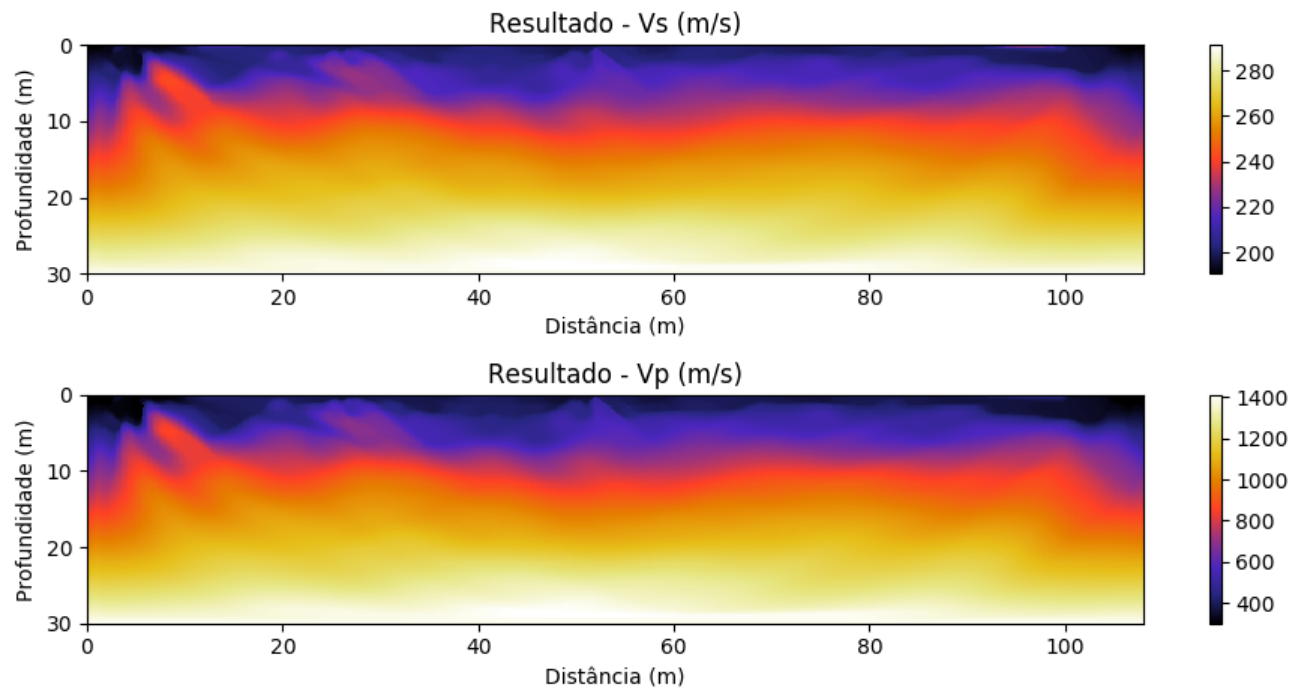

Figura 36 - Resultado em detalhe extraído em diferentes posições dos modelos da Figura 35.
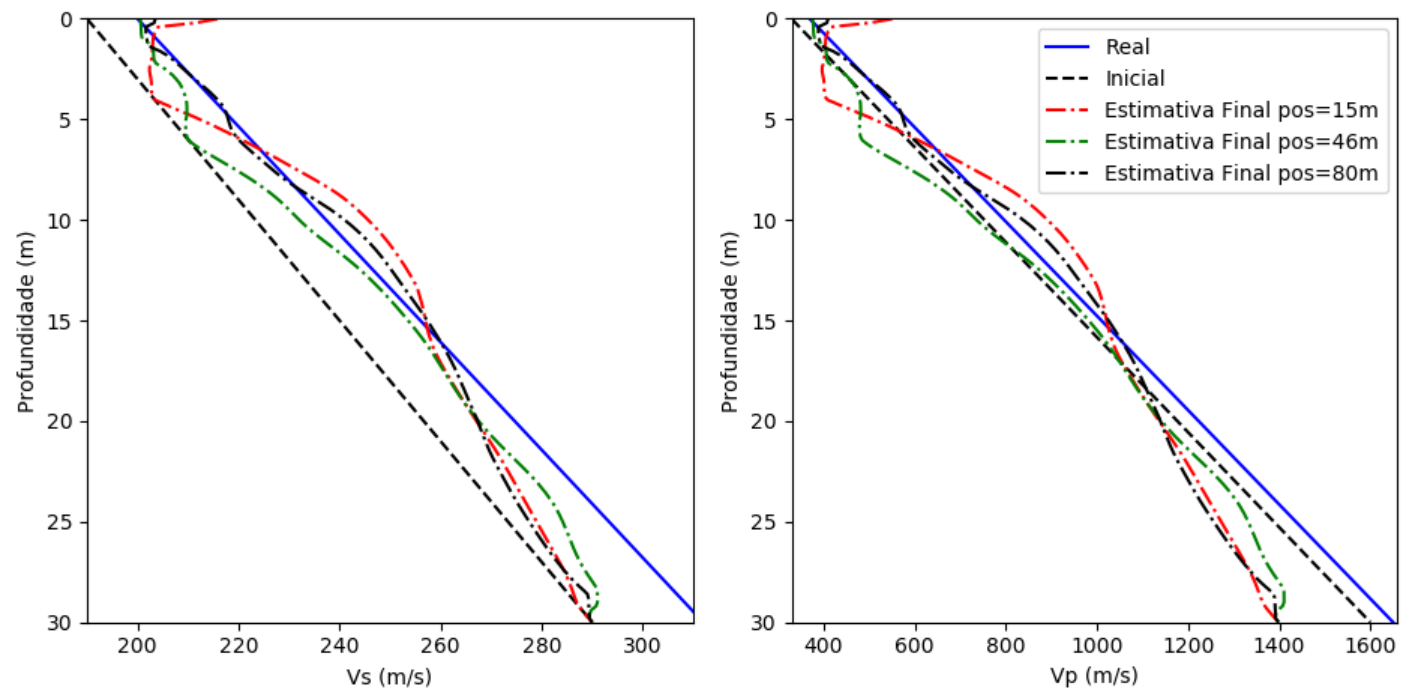
Figura 37 - Resultado para a estimativa concomitante da velocidade das ondas P e S e da densidade no modelo de camadas em uma aquisição com três pontos de tiro nas posições de 5,28 e 52 metros.
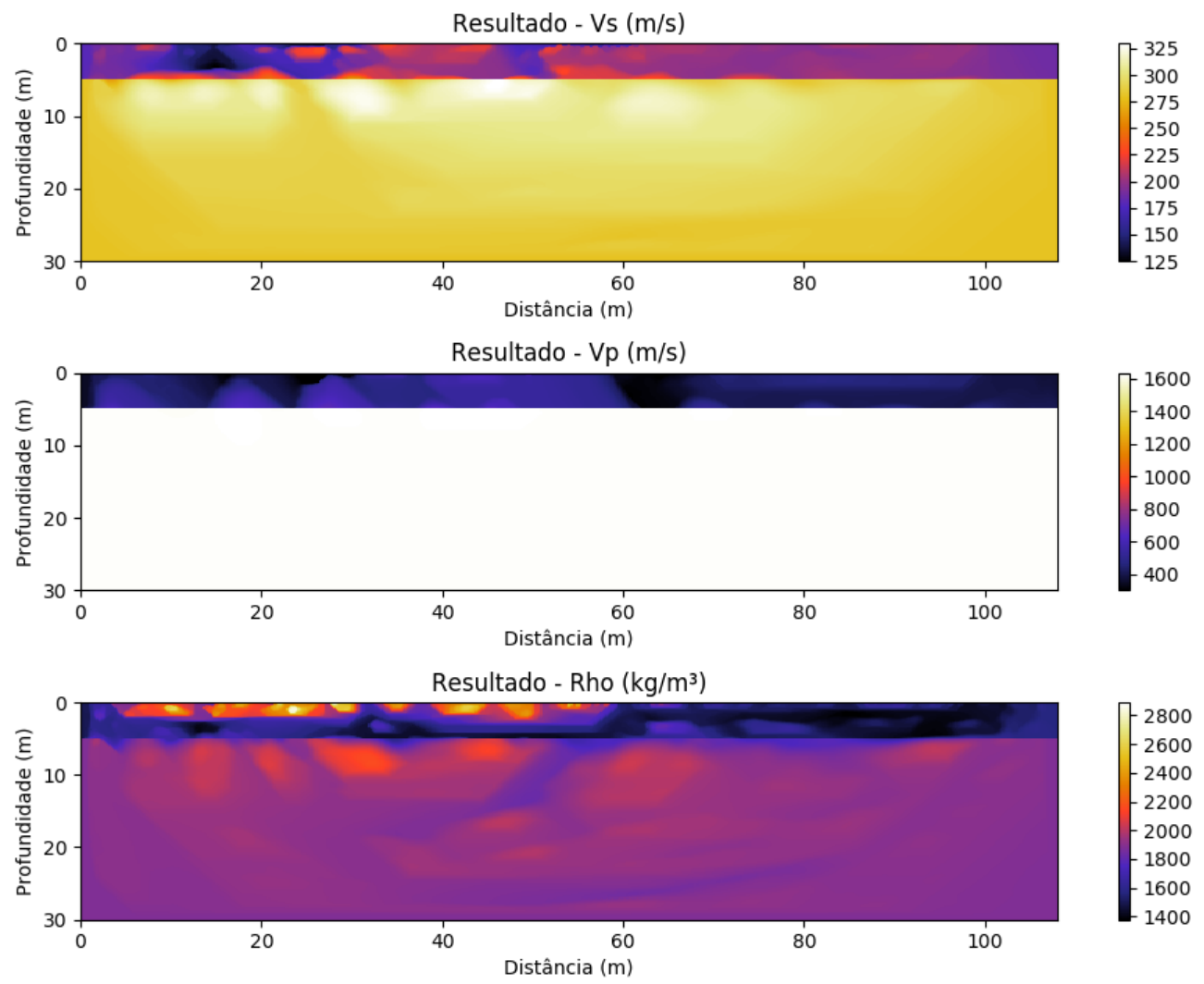
Quando os resultados da Figura 37 são observados nos detalhes apresentados na Figura 38 fica claro que todos os modelos possuem influência na evolução da resposta. Entretanto, a velocidade da onda S é o parâmetro que sofre menor influência no caso de valores errados nos demais modelos. Já a densidade apresenta grande variabilidade na resposta final.

No caso dos modelos de crescimento linear (Figuras 39 e 40) a resposta também apresenta maior variabilidade para a densidade e valores de $V_{P}$ e $V_{S}$ mais próximos dos desejados.

Figura 38 - Resultado em detalhe extraído em diferentes posições dos modelos da Figura 37.
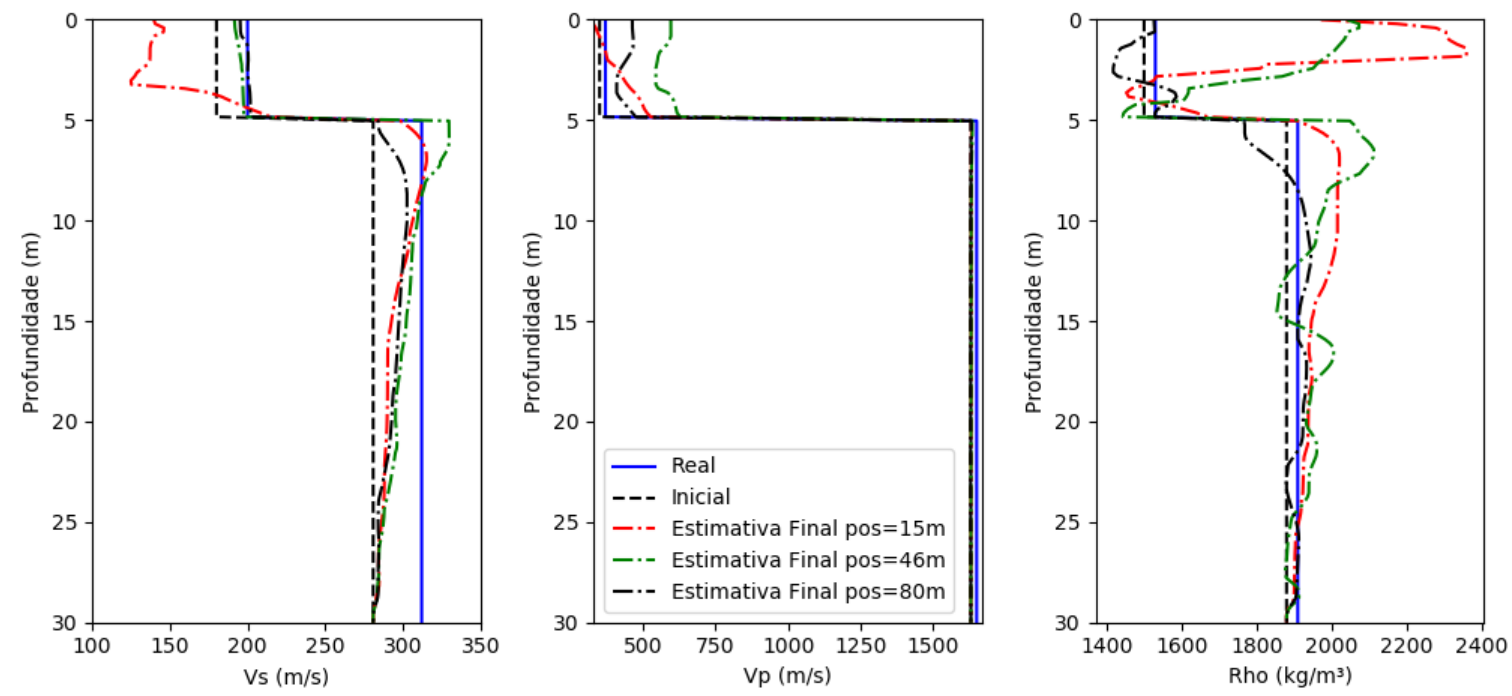
Figura 39 - Resultado para a estimativa concomitante da velocidade das ondas $\mathrm{P}$ e $\mathrm{S}$ e da densidade no modelo com crescimento linear de parâmetros em uma aquisição com três pontos de tiro nas posições de 5,28 e 52 metros.
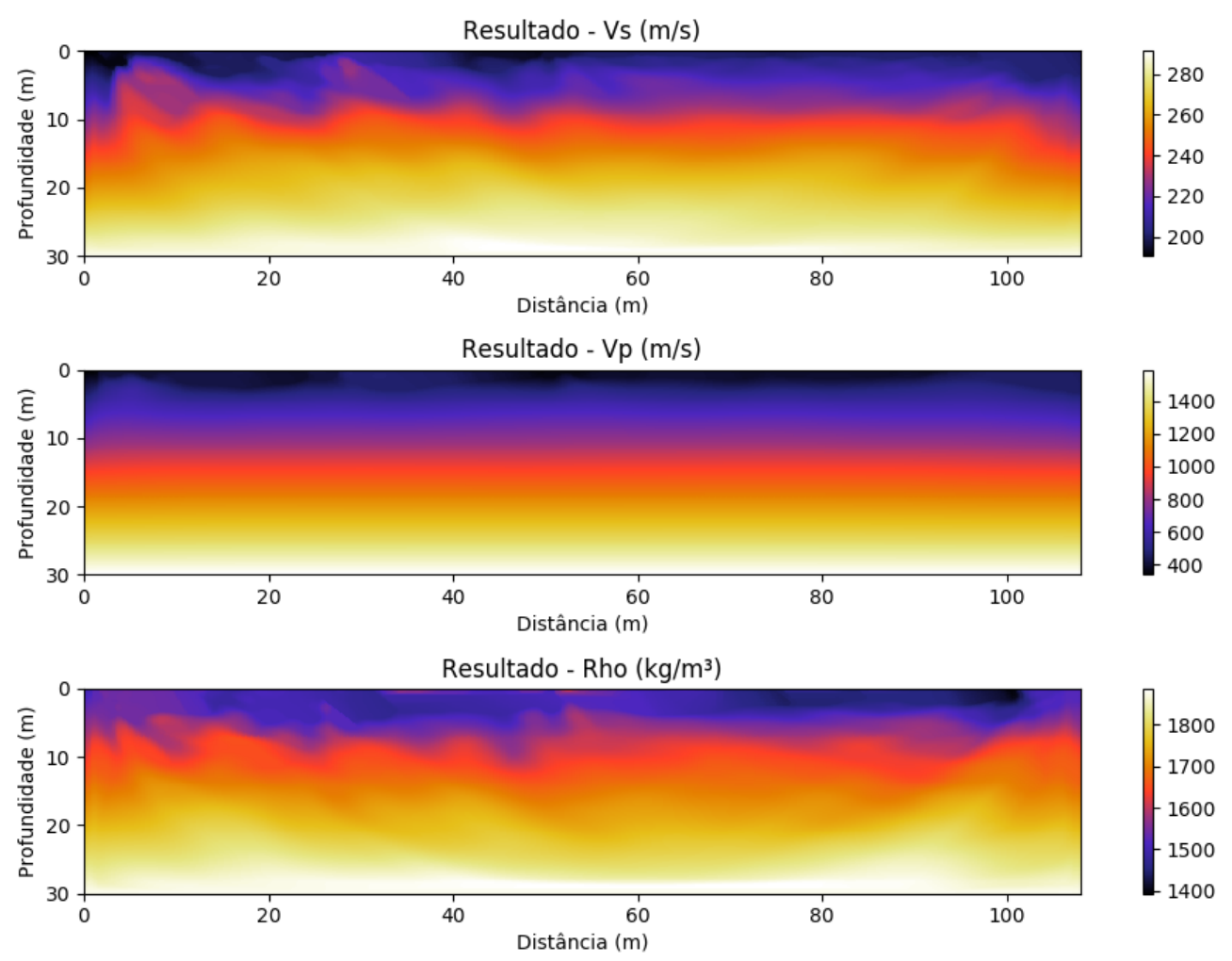

Figura 40 - Resultado em detalhe extraído em diferentes posições dos modelos da Figura 39.
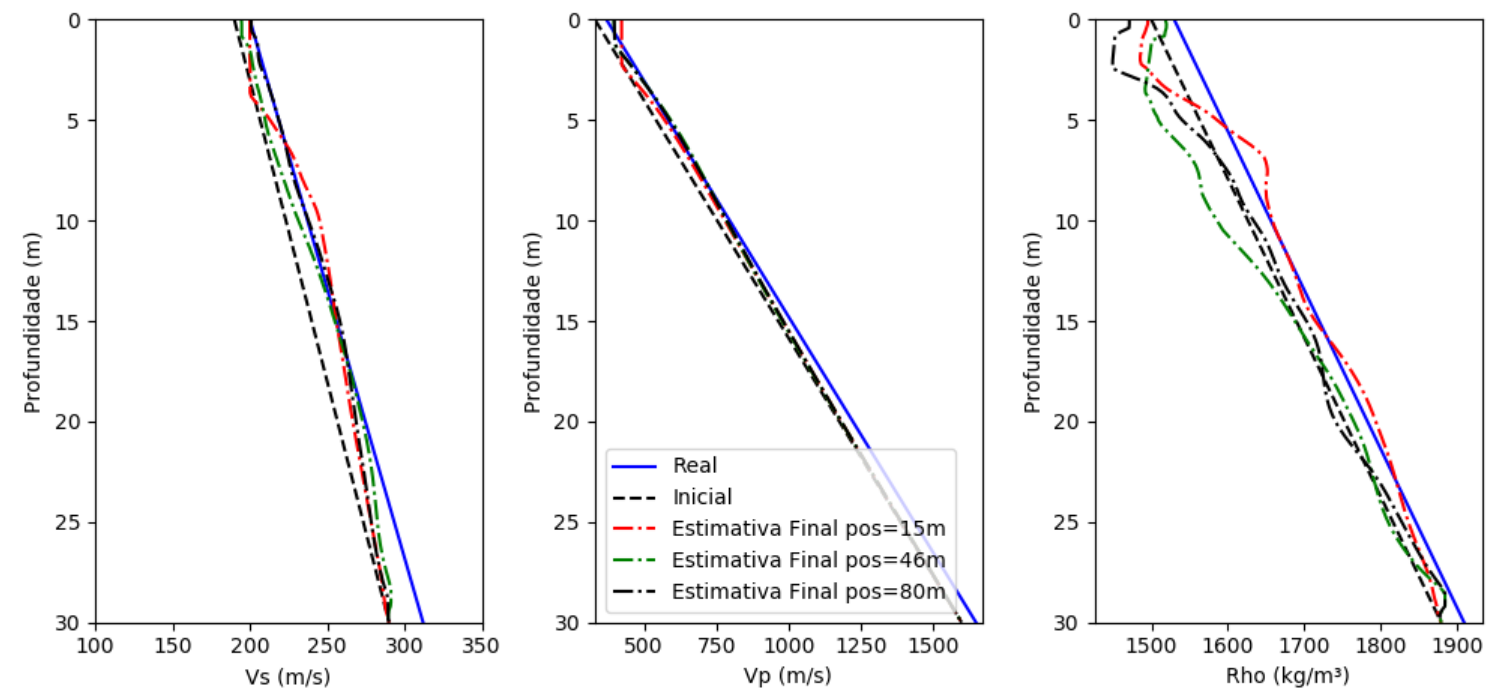

No caso de utilizar relações entre parâmetros (Equações 3.8 e 3.9) novamente foram observados os problemas de heterogeneidades do modelo $V_{S}$ nos demais modelos (Figuras 41 a 44). Estas figuras são os resultados $2 \mathrm{D}$ da estimativa da $V_{S}$ conjuntamente com a $V_{P}$ e a densidade. Onde a $V_{P}$ e a densidade são estimadas pelas equações 3.8 e 3.9. Entretanto, 
a utilização deste tipo de relação entre os parâmetros pode permitir a convergência da $V_{S}$ fazendo com que os demais modelos sejam atualizados de forma adequada. Como por exemplo pequenas modificações na posição de uma interface.

Figura 41 - Resultado para a estimativa concomitante da velocidade das ondas $\mathrm{P}$ e S e da densidade no modelo de camadas, onde Vp e a densidade são estimadas através de uma relação com Vs, em uma aquisição com três pontos de tiro nas posições de 5,28 e 52 metros.
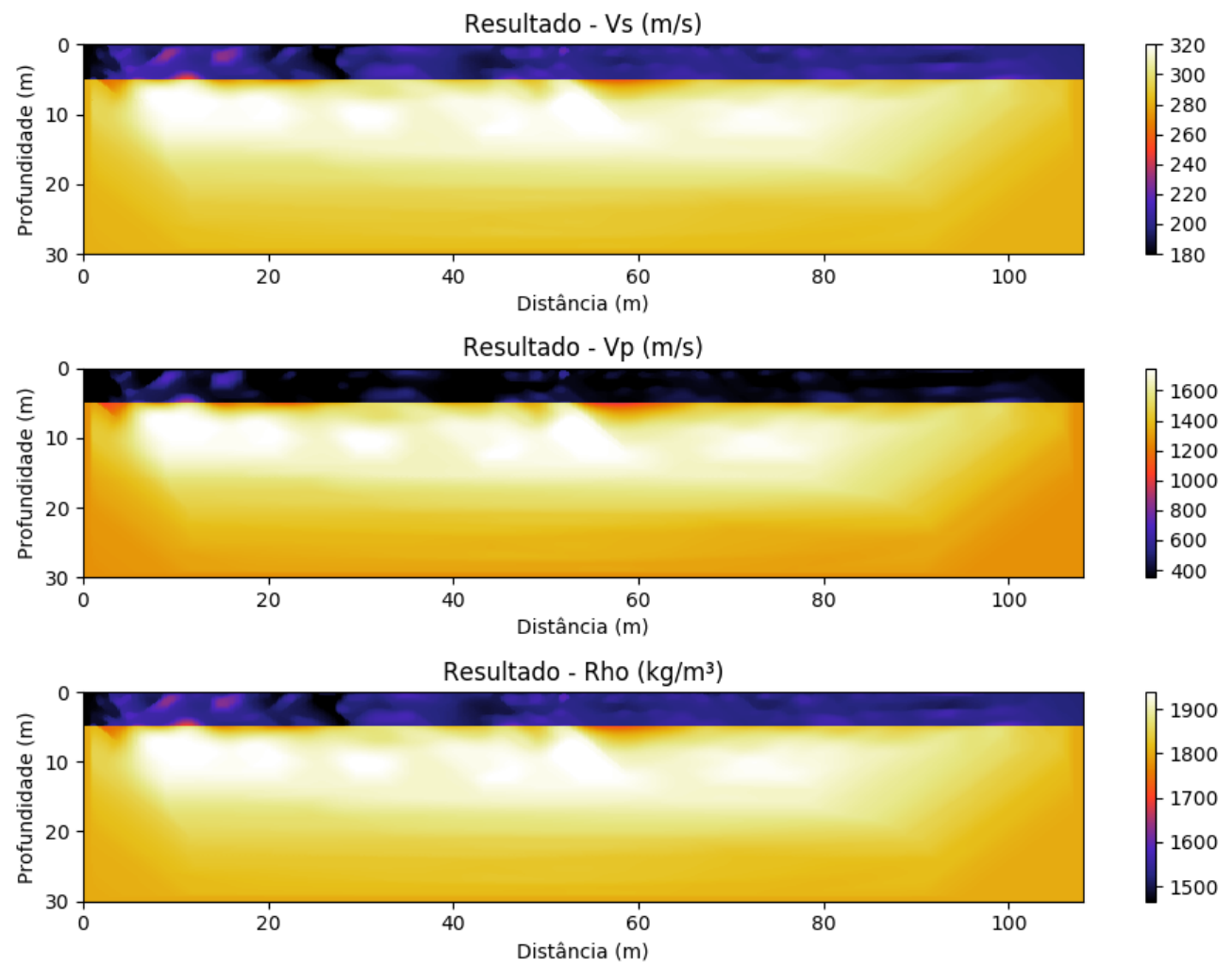
Figura 42 - Resultado em detalhe extraído em diferentes posições dos modelos da Figura 41.
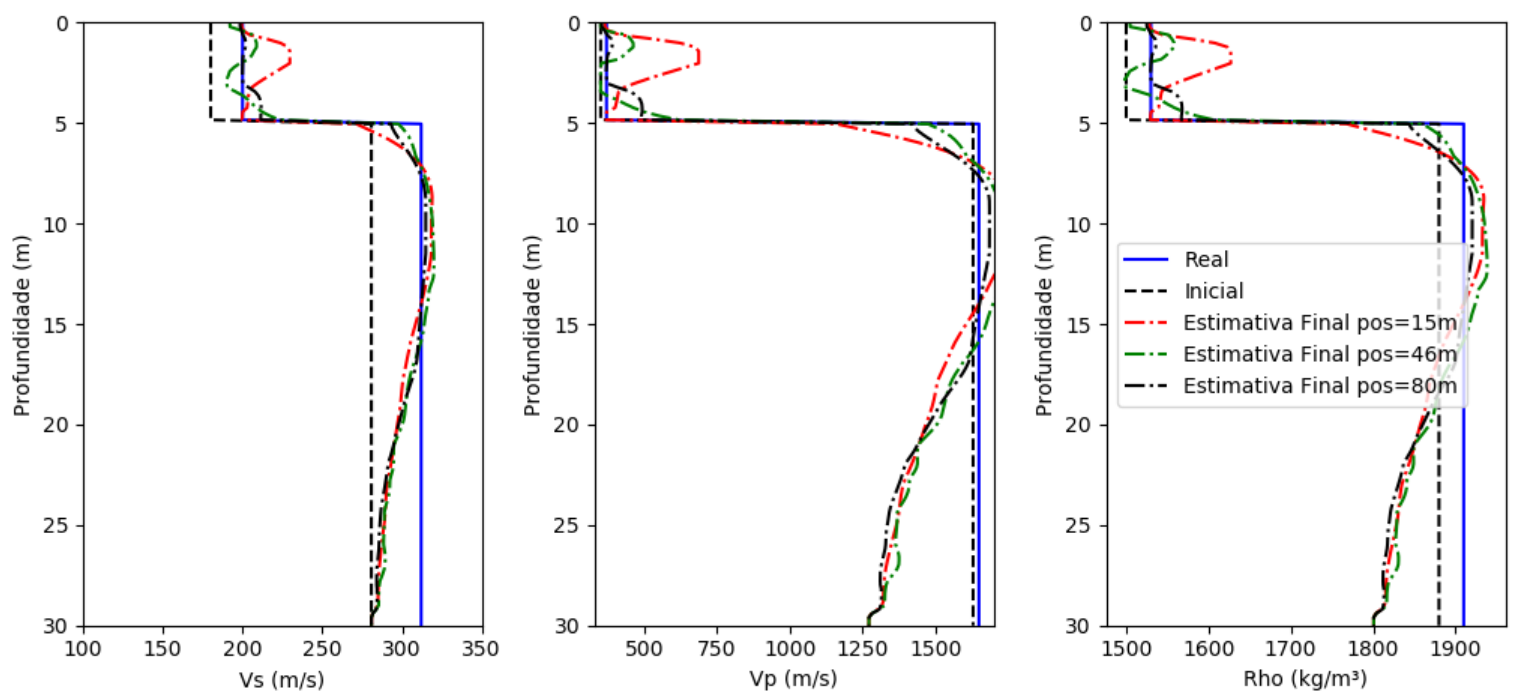

Figura 43 - Resultado para a estimativa concomitante da velocidade das ondas $\mathrm{P}$ e $\mathrm{S}$ e da densidade no modelo com crescimento linear de parâmetros e relação de Vp e densidade com Vs em uma aquisição com três pontos de tiro nas posições de 5,28 e 52 metros.
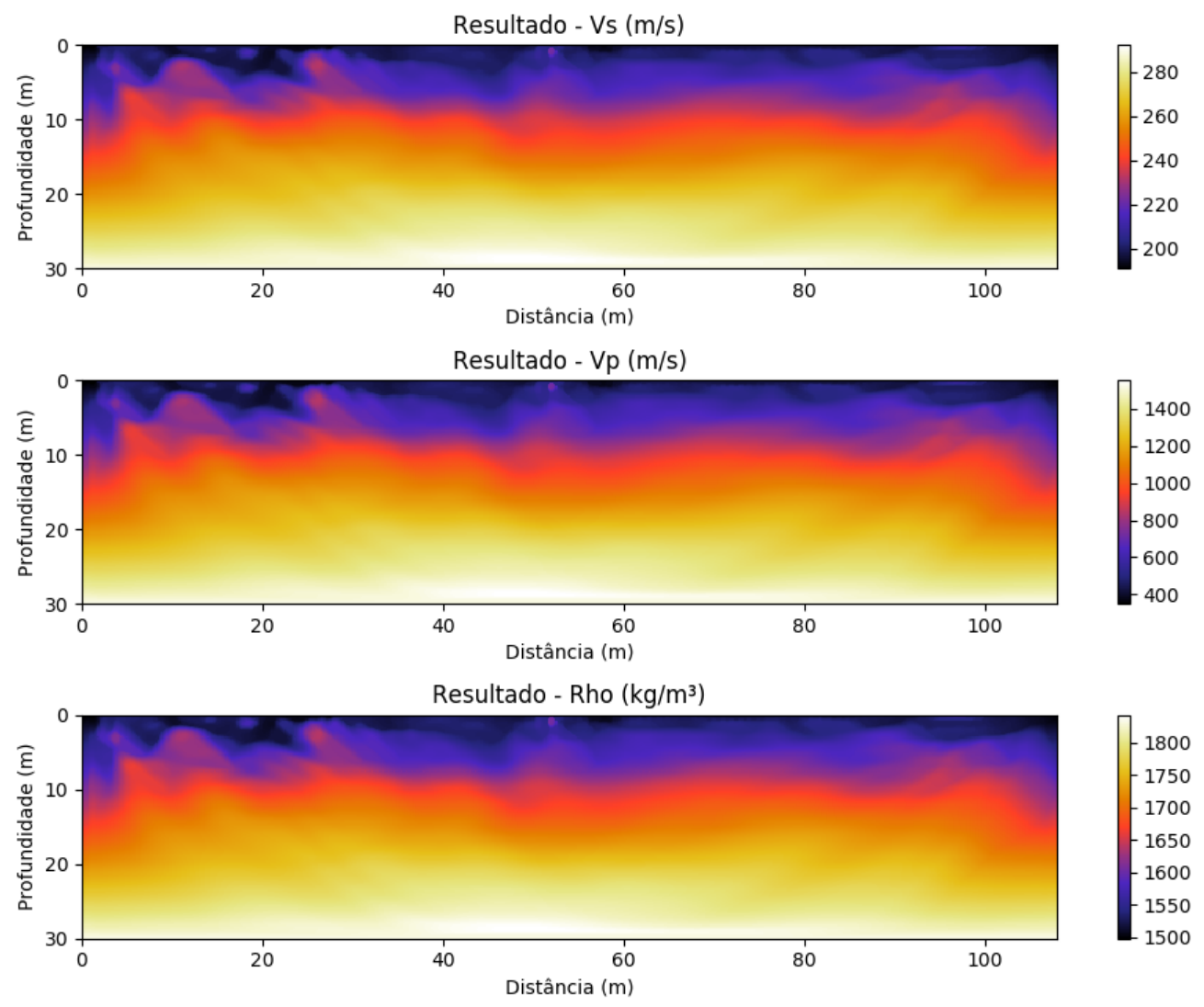
Figura 44 - Resultado em detalhe extraído em diferentes posições dos modelos da Figura 43.
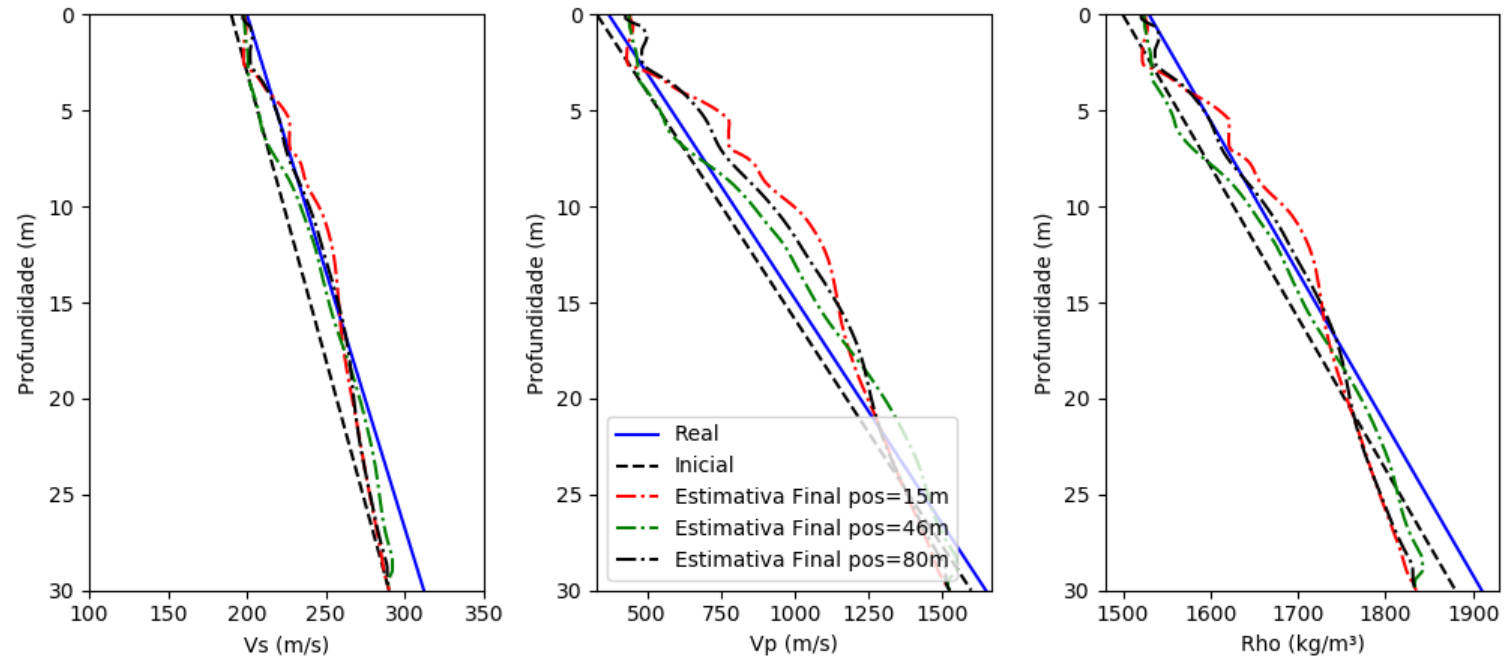


\subsubsection{Manipulação do gradiente}

Segundo Mengxuan et al. (2017) o gradiente calculado em FWI é influenciado pela difusão geométrica e pela iluminação desigual tanto na propagação quanto na retro propagação dos campos de onda. O objetivo neste tópico foi estudar os efeitos do gradiente na resposta final obtida. Assim, o foco das inversões foi no parâmetro $V_{S}$, mantendo os demais parâmetros fixos e nos valores corretos em relação ao modelo real.

\subsubsection{Afunilamento do gradiente}

Definindo o termo taper como afunilamento, existem algumas formas que podem ser utilizadas para o afunilamento do gradiente (Figura 45). Este procedimento é necessário já que como mencionado em Groos (2013) os gradientes possuem altas amplitudes na vizinhança das fontes. Aqui foi aplicado um filtro de afunilamento circular em torno das posições de tio. De forma que os gradientes de cada tiro fossem pré-condicionados com uma janela que afeta apenas a janela do tiro em questão. Estes filtros circulares possuem valor unitário na borda do filtro até o valor nulo na posição do tiro. A forma utilizada para o filtro corresponde a uma função de erro.

Figura 45 - Pré-condicionamento do taper para um tiro na posição $\mathrm{x}=25 \mathrm{~m}$ na superfície livre. (a) modelo completo; (b) região do taper em detalhe.

(a)

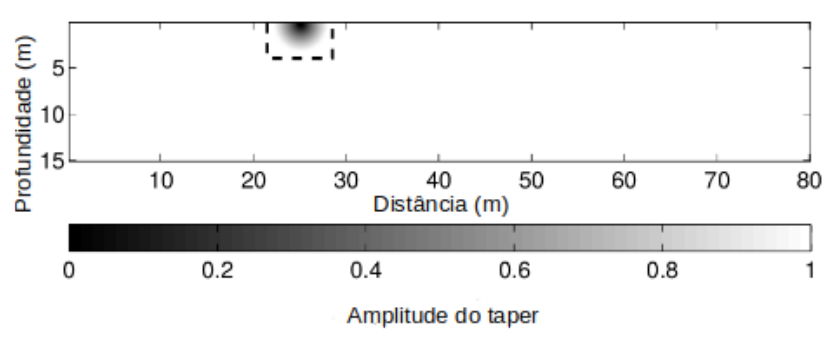

Fonte: Groos (2013). (b)

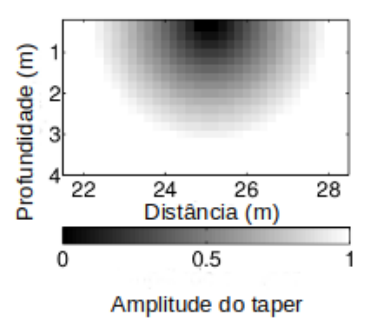

O parâmetro que pode ser utilizado para controlar o filtro é o tamanho do raio. Aqui foram testados tamanhos de raios diferentes.

Nos resultados apresentados nas Figuras 46 e 47 observa-se pouca melhoria na resposta através da utilização de um raio diferente para o filtro de taper. Entretanto, o raio de tamanho intermediário aparenta retornar modelos com menos efeitos indesejáveis e menor amplitudes na região das fontes. Raios maiores iriam prejudicar a resposta obtida nas camadas de menor profundidade, já que neste caso o gradiente não seria atualizado nesta parte do modelo. 
Figura 46 - Resultado para a estimativa da velocidade da onda S no modelo de camadas em uma aquisição com três pontos de tiro nas posições de 5,28 e 52 metros. Cada resultado foi obtido para um valor diferente raio diferente para o afunilamento do gradiente na posição das fontes.
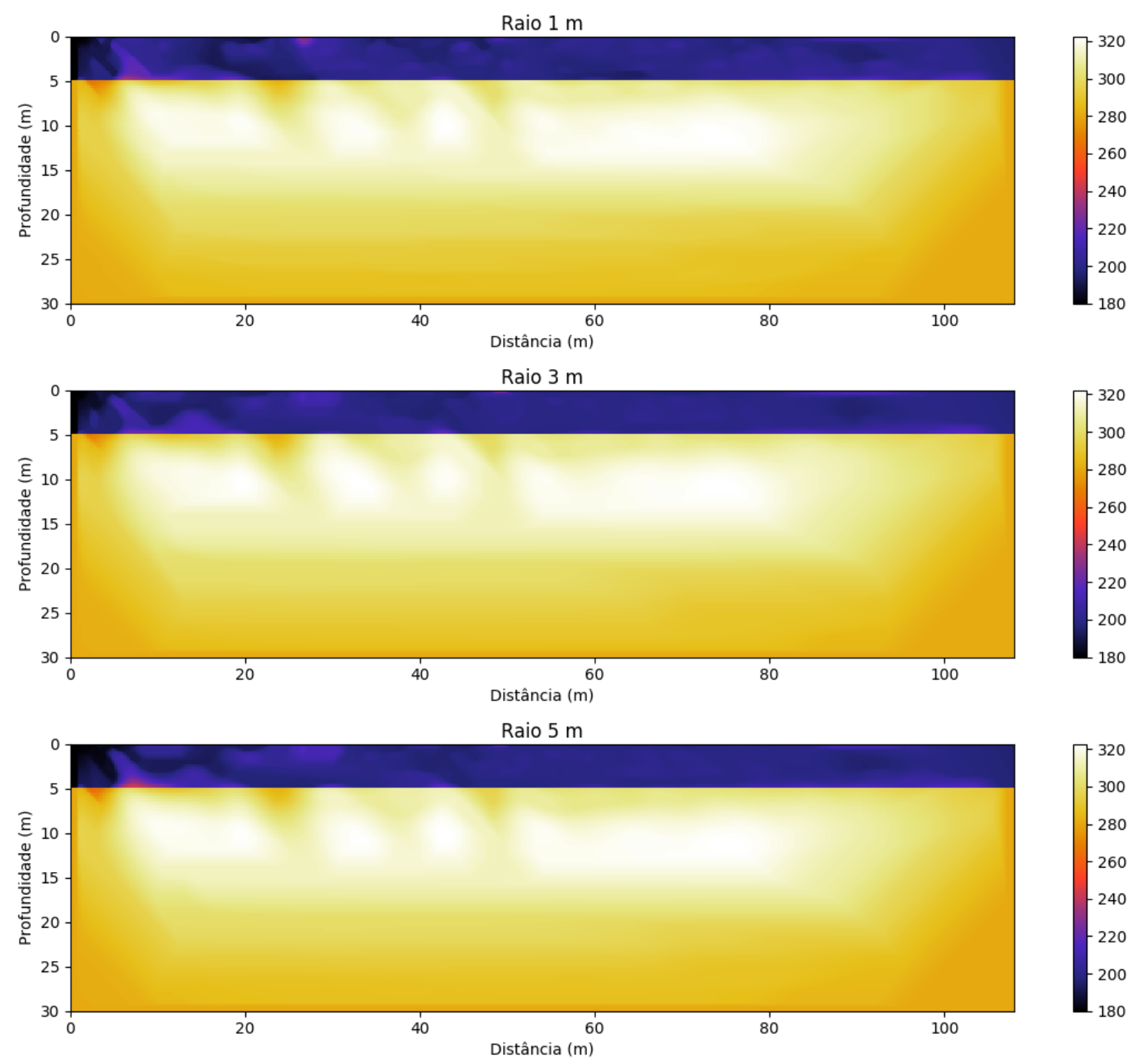
Figura 47 - Resultado para a estimativa da velocidade da onda S no modelo de onde os parâmetro crescem linearmente com a profundidade em uma aquisição com três pontos de tiro nas posições de 5,28 e 52 metros. Cada resultado foi obtido para um valor diferente raio diferente para o afunilamento do gradiente na posição das fontes.
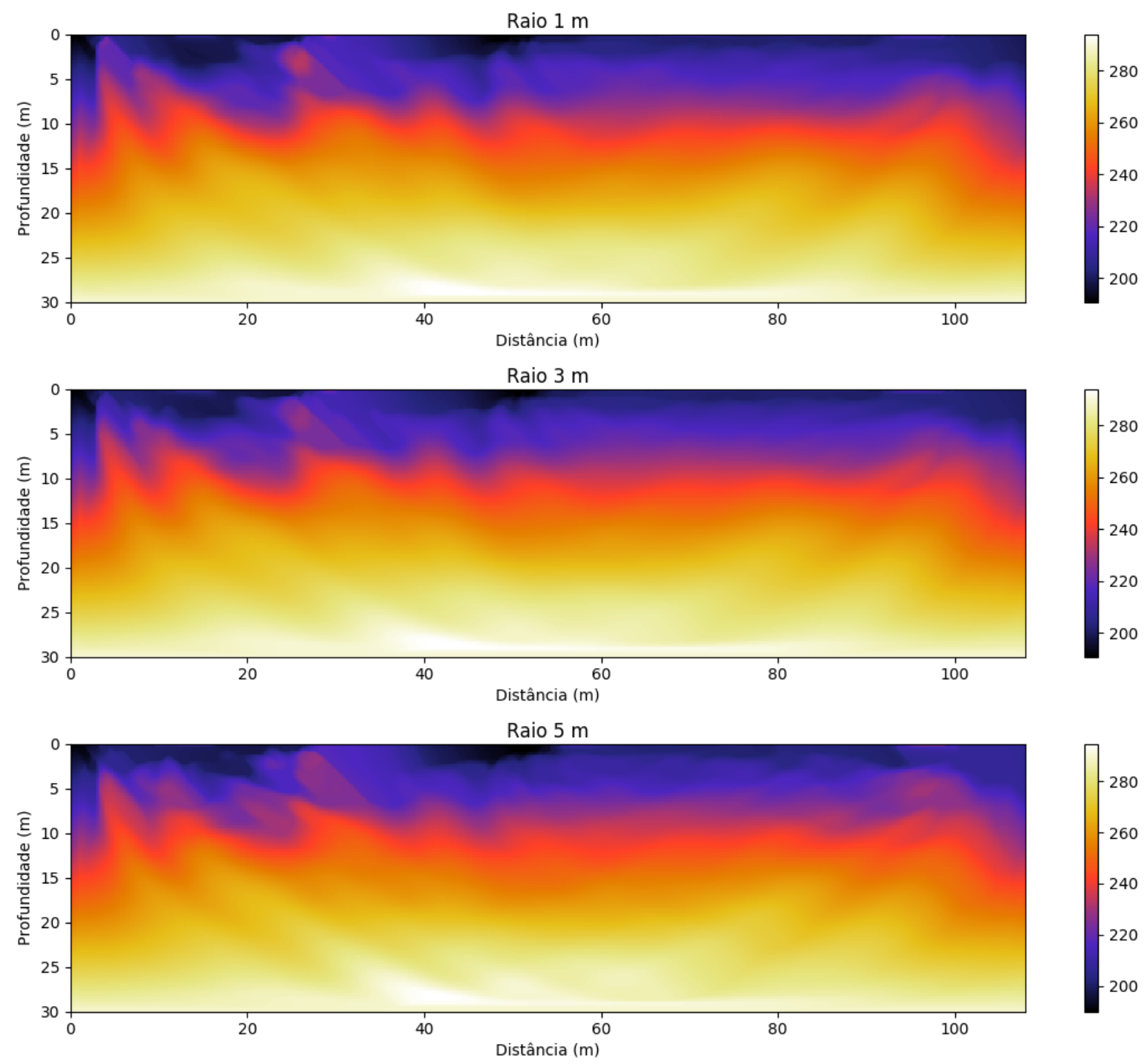


\subsubsection{Efeito do pré condicionamento do gradiente - Controle da aproximação da Hessiana}

Em todos os resultados anteriores não havia sido utilizada a opção de pré condicionamento do gradiente no código IFOS2D. Aqui foi utilizada uma aproximação da Hessiana para o pré-condicionamento do gradiente, assim como descrito no tópico 3.1.6 do capítulo 3. Foram avaliados os resultados obtidos com a técnica apresentada em Shin et al. (2001) e em Plessix e Mulder (2004), estas serão chamadas de técnicas H1 e H3 para brevidade. Os resultados mostrados aqui foram obtidos assumindo diferentes "níveis d' água"utilizados para estabilizar a Hessiana aproximada.

Figura 48 - Resultado para a estimativa da velocidade da onda S no modelo de camadas em uma aquisição com três pontos de tiro nas posições de 5,28 e 52 metros. Cada resultado com a Hessiana estimada por H1 com um valor de "nível d'água" diferente.
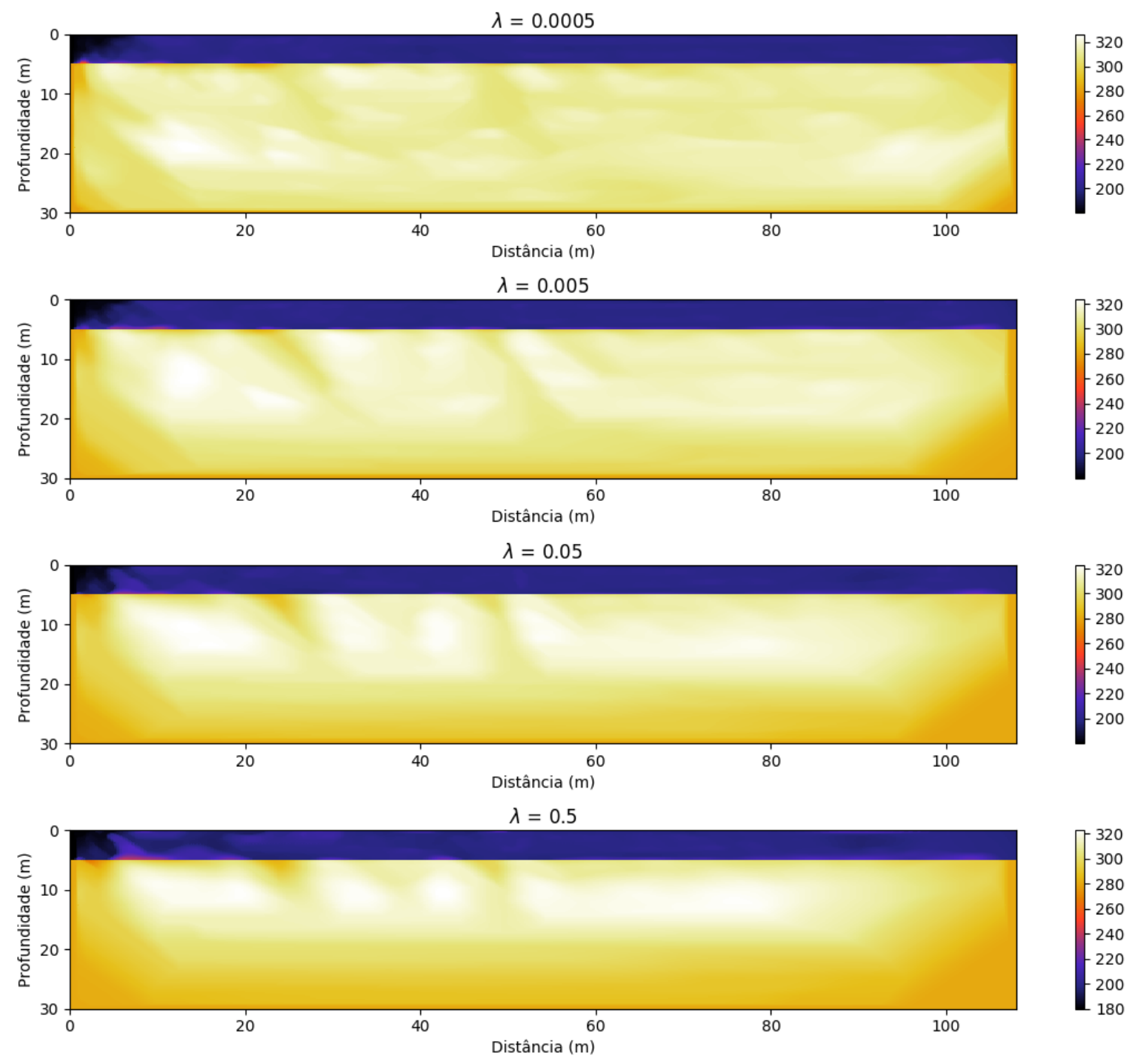
Figura 49 - Resultado em detalhe extraído em diferentes posições dos modelos da Figura 48.
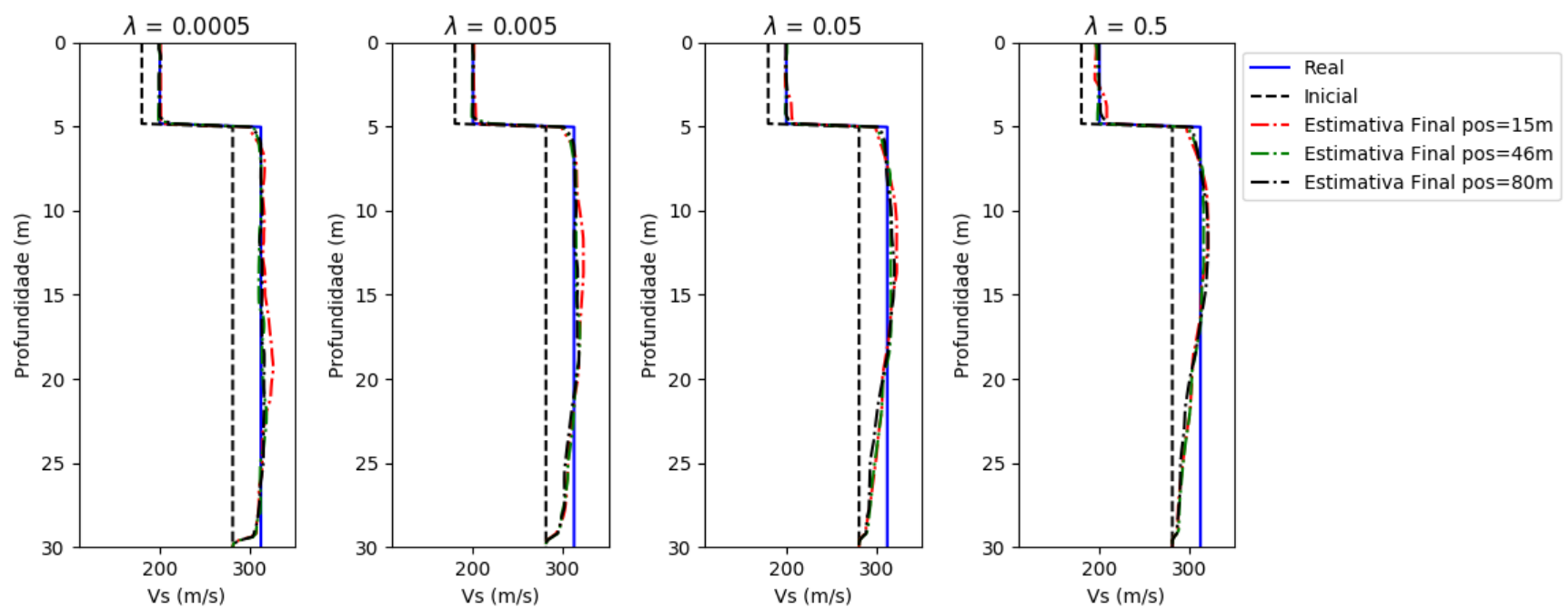
Figura 50 - Resultado para a estimativa da velocidade da onda S no modelo de crescimento linear dos parâmetros com a profundidade em uma aquisição com três pontos de tiro nas posições de 5,28 e 52 metros. Cada resultado com a Hessiana estimada por H1 com um valor de "nível d'água" diferente.
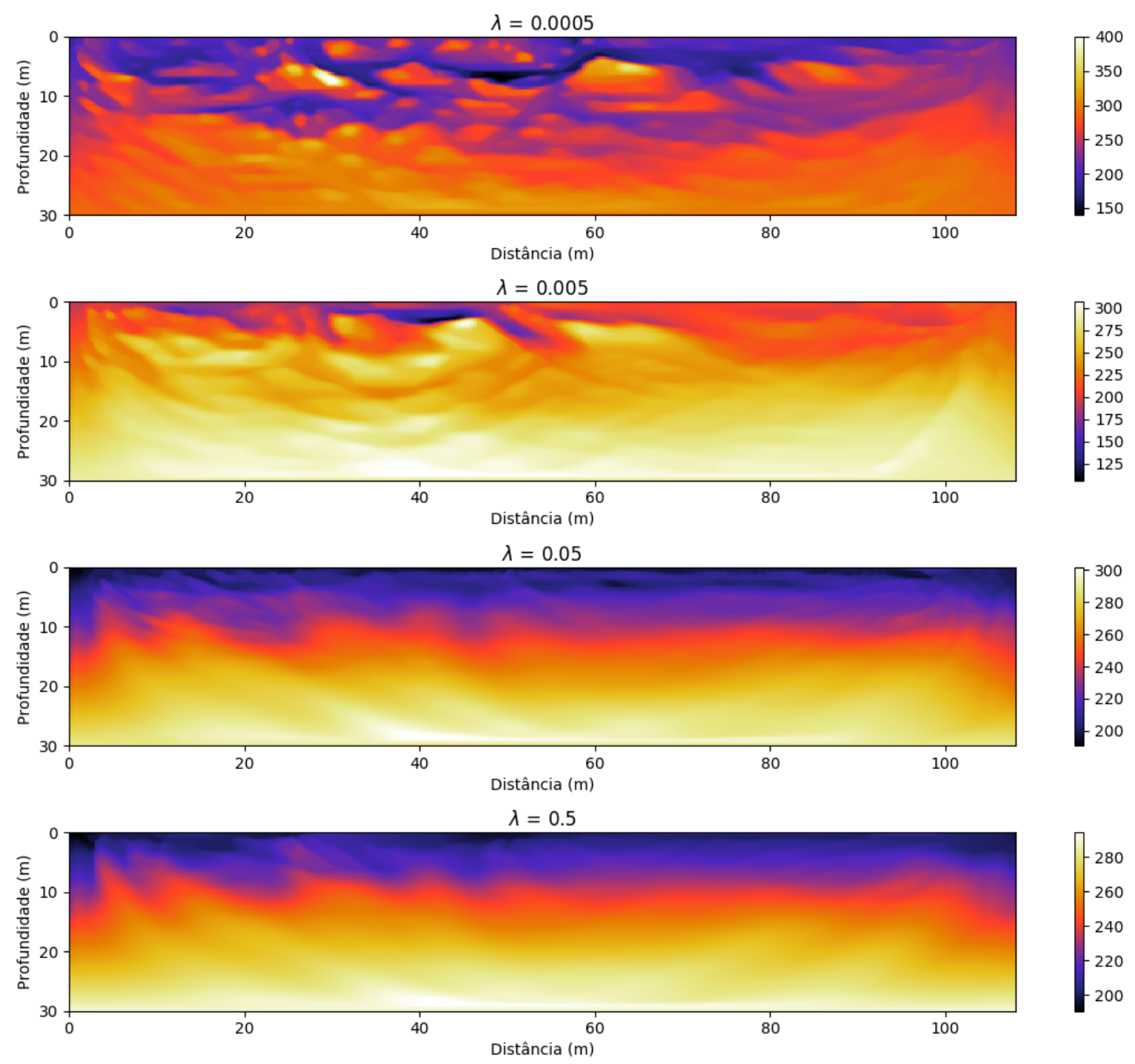
Figura 51 - Resultado em detalhe extraído em diferentes posições dos modelos da Figura 50.
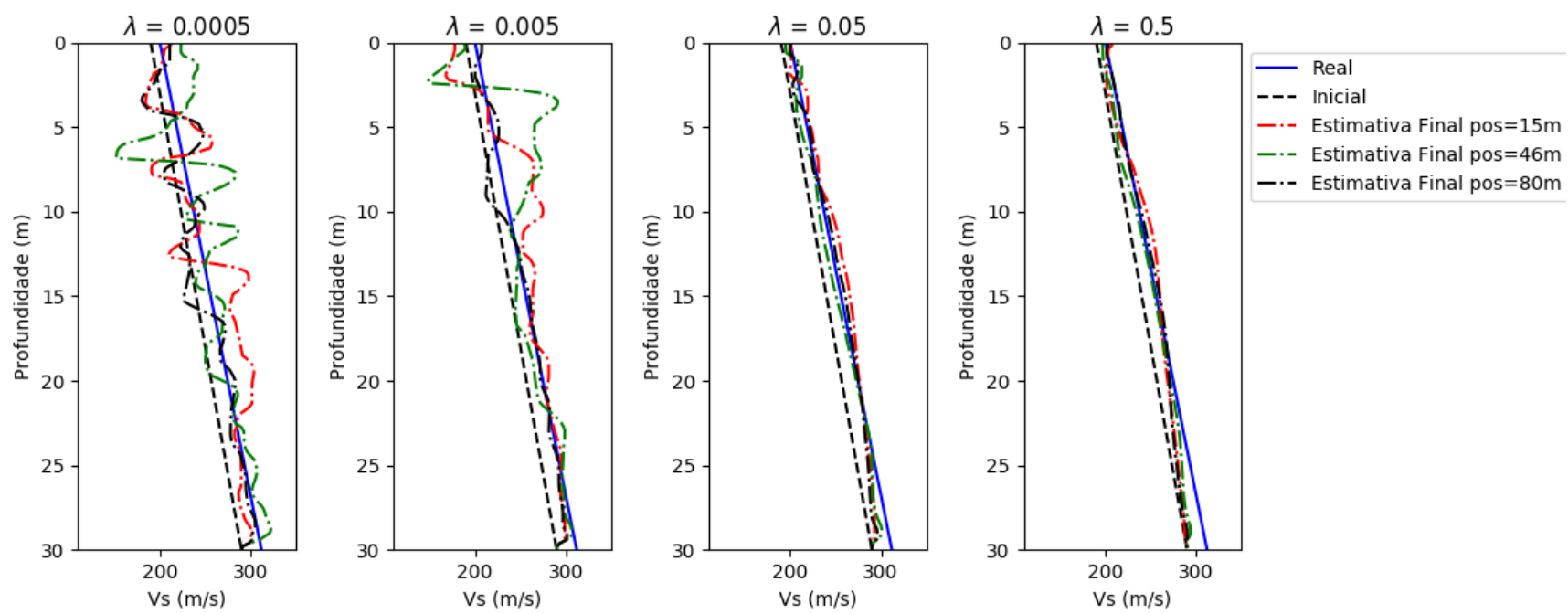
Figura 52 - Resultado para a estimativa da velocidade da onda S no modelo de camadas em uma aquisição com três pontos de tiro nas posições de 5,28 e 52 metros. Cada resultado com a Hessiana estimada por H3 com um valor de "nível d'água" diferente.
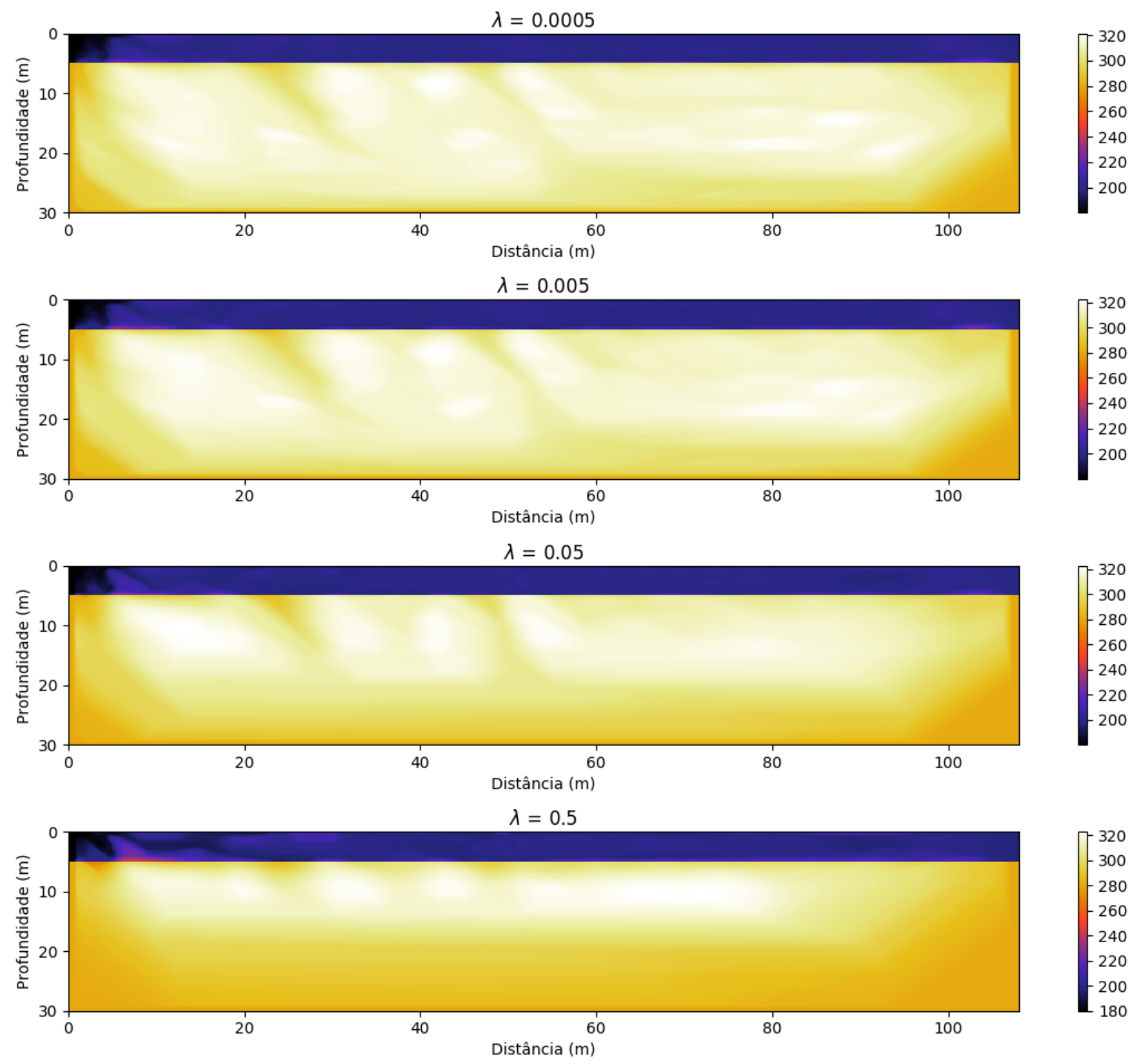
Figura 53 - Resultado em detalhe extraído em diferentes posições dos modelos da Figura 52.
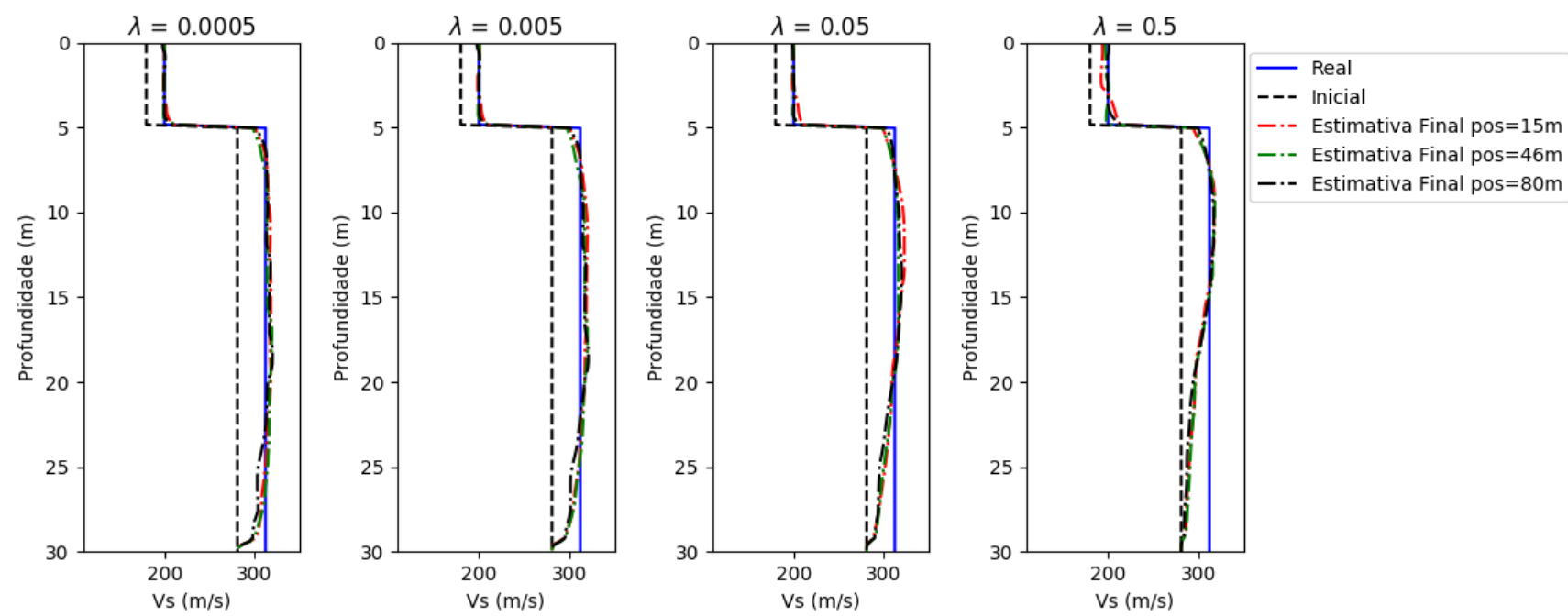
Figura 54 - Resultado para a estimativa da velocidade da onda S no modelo de crescimento linear dos parâmetros com a profundidade em uma aquisição com três pontos de tiro nas posições de 5,28 e 52 metros. Cada resultado com a Hessiana estimada por H3 com um valor de "nível d'água" diferente.
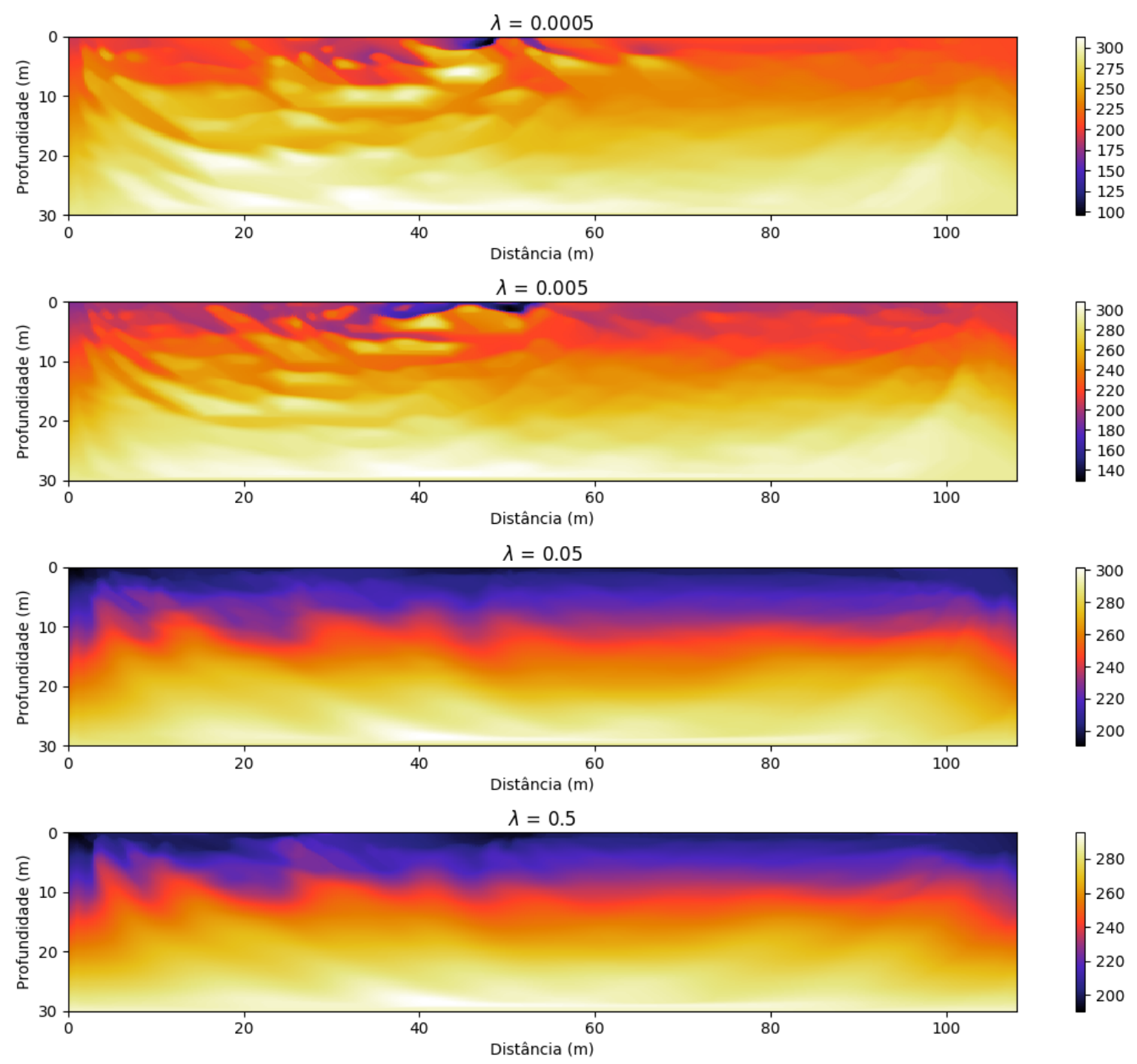
Figura 55 - Resultado em detalhe extraído em diferentes posições dos modelos da Figura 54.
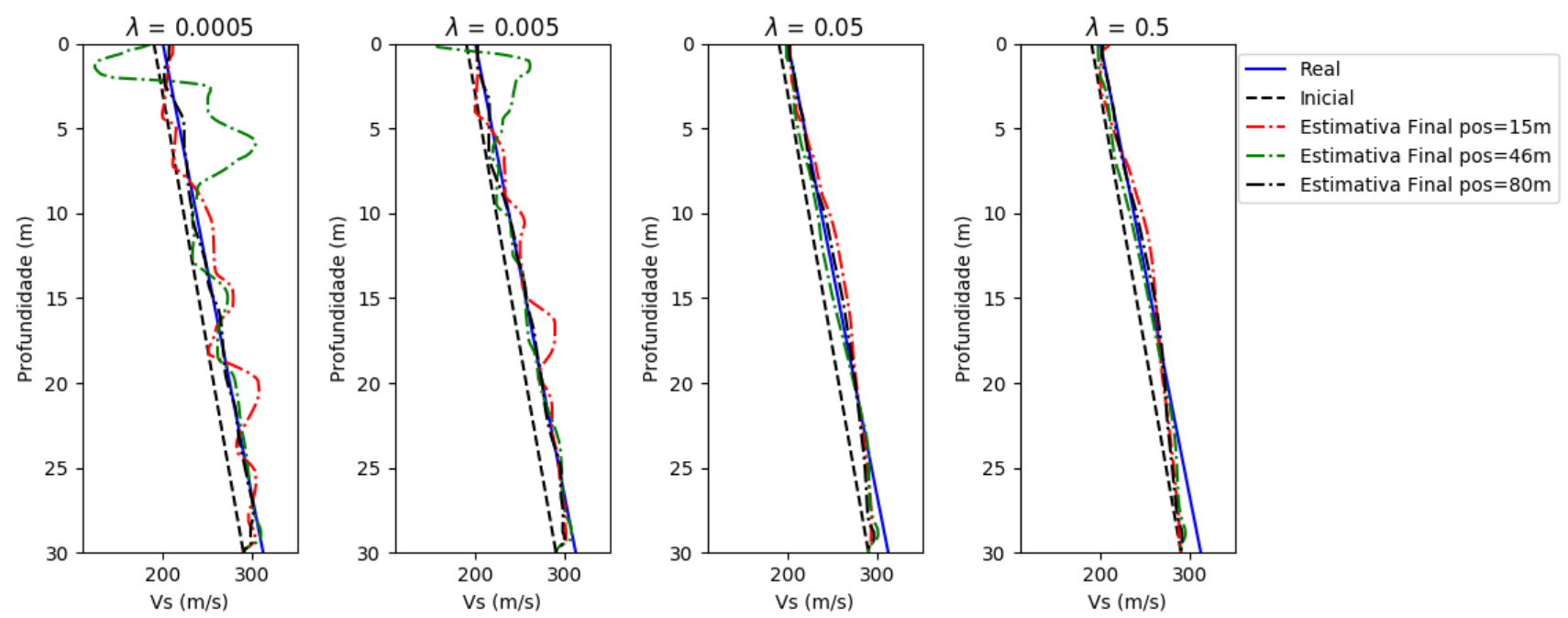

Os resultados obtidos por H1 e H3 são diferentes. Entretanto, a diferença observada é subjetiva. Não existe algo nas imagens que possa indicar que um tipo de aproximação da Hessiana funciona melhor que o outro pelos exemplos testados.

$\mathrm{O}$ valor de $\lambda$ escolhido mostra grande influência na resposta final. A utilização da Hessiana aproximada em conjunto com a soma de um valor constante na diagonal principal da Hessiana funciona como uma regularização do problema. Logo, um peso grande para a regularização irá retornar um modelo final mais grosseiro ou muito próximo da estimativa inicial. Um peso pequeno para a regularização pode ser capaz de retornar pequenos detalhes do modelo real, mas fica mais suscetível a instabilidades.

\subsubsection{Modelo com camada inclinada}

Neste teste foi simulado um levantamento de reflexão com vários tiros. Foi realizada a estimativa da velocidade da onda $\mathrm{S}$, mantendo os modelos de $V_{P}$ e densidade fixados na estimativa inicial. Foram utilizados 24 pontos de tiro no intervalo de 5 até 28 metros e 48 geofones posicionados no intervalo de 29 até 76 metros. É importante notar que para este teste os modelos reais de $V_{P}$ e densidade se assemelham ao modelo real $V_{S}$ na Figura 56. Da mesma forma os modelos iniciais $V_{P}$ e densidade também apresentam uma interface plana na mesma profundidade da $V_{S}$ inicial. Embora bastante dependente do modelo inicial foi possível notar no resultado final um aumento dos valores relacionado a inclinação do modelo real. 
Figura 56 - Resultados para a estimativa da velocidade da onda S para um modelo real de camada inclinada.
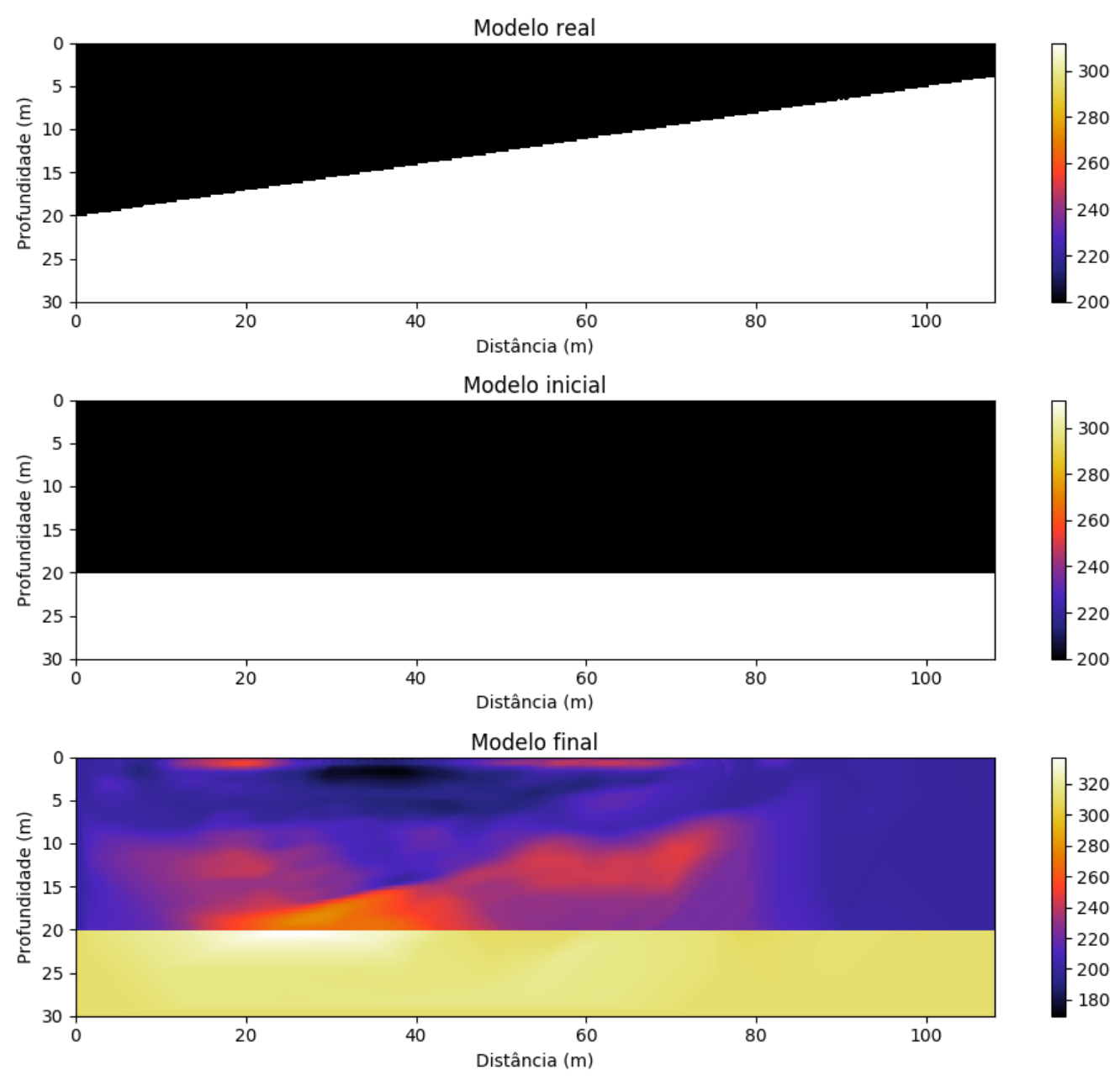


\section{Aquisição e Processamento P-Sv}

As ondas sísmicas se dividem em dois tipos principais, as ondas de corpo e as ondas superficiais. As ondas de corpo se dividem ainda em ondas compressionais e de cisalhamento. As ondas compressionais possuem movimento da partícula no mesmo sentido da propagação da onda e são chamadas de ondas $\mathrm{P}$ e as ondas de cisalhamento, chamadas de ondas $\mathrm{S}$, tem o movimento da partícula transversal ao sentido de propagação da onda. Ao atingir uma interface entre duas camadas geológicas pode haver conversão de P para S ou vice-versa.

Já as ondas superficiais se dividem em dois tipos as ondas Love e as ondas Rayleigh. Sendo as ondas Love uma combinação de ondas S de componente horizontal (SH) e as ondas Rayleigh uma combinação entre as ondas P e S de componente vertical (SV). As ondas superficiais observadas em aquisições sísmicas são chamadas de ground-roll e este é composto primordialmente pelas ondas Rayleigh.

\subsection{Aquisição}

Neste trabalho foram analisados sismogramas adquiridos com geofones de componente vertical e radial (Figura 57). Nestes sismogramas são esperados eventos de reflexão PP (observados principalmente na componente vertical) e PS (observados principalmente na componente radial) além de eventos de onda direta, refração e ground-roll.

Figura 57 - Geofones de componente vertical (esquerda) e componente radial (direita).
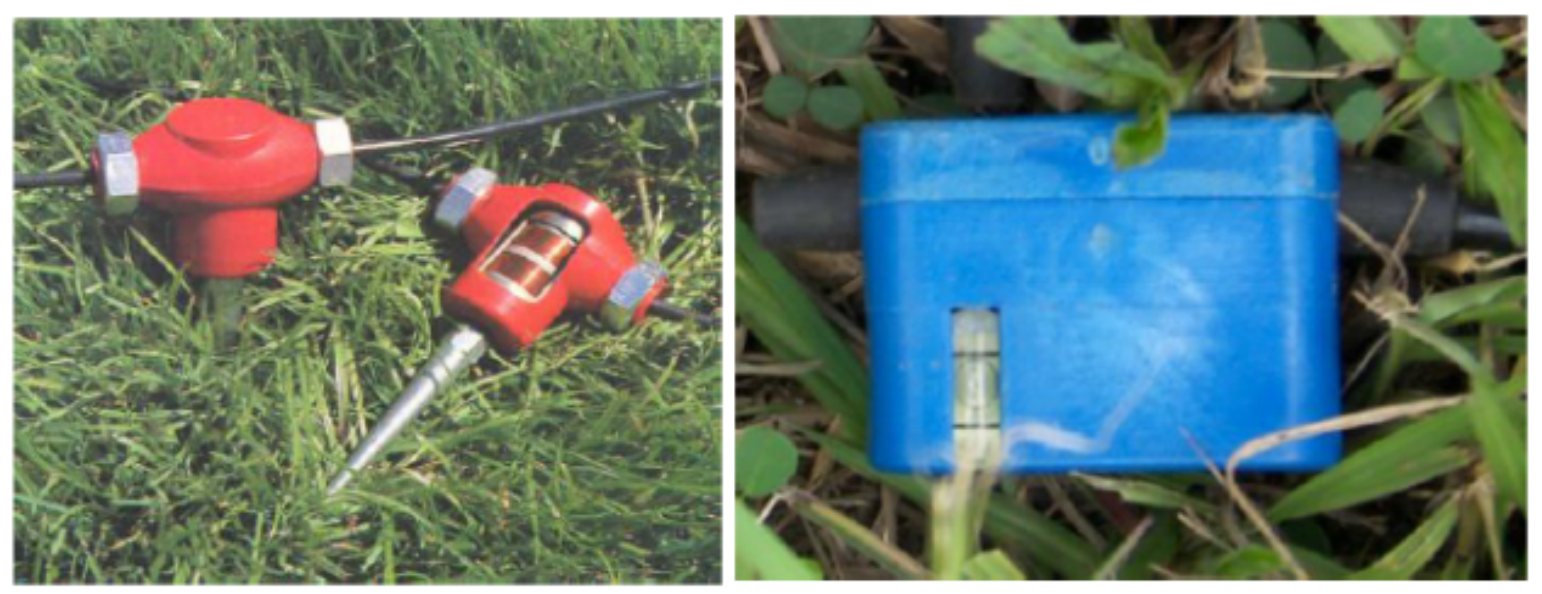

Fonte: Bokhonok (2010) 
Em aquisições do tipo multicomponente, onde o objetivo é a aquisição de reflexões PP e PS, são utilizadas fontes essencialmente monopolares. Uma fonte monopolar irradia energia igualmente em todas as direções e é considerada como uma fonte que gera apenas a onda P. Esta consideração é feita mesmo sabendo que uma fonte que realiza movimento vertical é capaz de gerar uma onda SV.

Para uma onda $\mathrm{P}$ incidente na interface entre dois meios diferentes, ocorre reflexão da onda em dois modos diferentes (P e S). Na Figura 58 é possível observar a assimetria da reflexão PS em relação à reflexão PP. A reflexão PS possui polarização perpendicular ao sentido de propagação da onda e por este motivo é mais bem registrada pelos geofones que medem a componente radial.

Além das componentes vertical e radial, a utilização de um terceiro geofone para a medição da componente horizontal, perpendicular à linha de aquisição, ou de geofones de três componentes. Se faz necessária na presença de refletores com mergulho, heterogeneidades e anisotropia.

Figura 58 - Onda P incidente e suas reflexões P e S em uma interface.

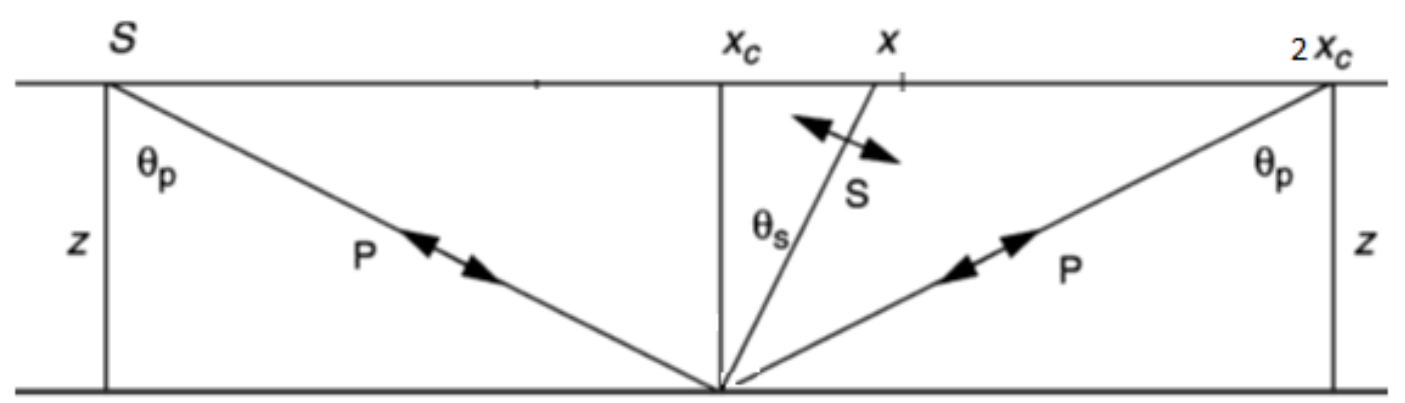

Fonte: Thomsen (1999).

\subsubsection{Técnica Mini-Sosie}

A técnica Mini-Sosie de Barbier (1983) utiliza um compactador de solos. Desta forma são produzidas vibrações aproximadamente aleatórias. Estas vibrações se propagam como um pulso sísmico de longa duração e baixa amplitude. O sinal emitido pela fonte (traço piloto) é registrado por um geofone posto próximo a fonte. Posteriormente é utilizada a correlação cruzada do traço piloto com cada traço sísmico. O resultado é equivalente ao sismograma adquirido com uma fonte que gerasse uma wavelet de fase zero.

Chamando um sismograma de reflexão de $s_{s}(t)$ é possível representá-lo como uma convolução de um pulso gerado pela fonte $w_{s}(t)$ com a refletividade $r(t)$ (resposta da Terra a um impulso), dado por: 


$$
s_{s}(t)=w_{s}(t) * r(t)+n(t)
$$

onde $n(t)$ é o ruído aleatório dos dados. Entretanto, na técnica mini-sosie o pulso $s_{s}(t)$ é gerado diversas vezes em um intervalo de tempo de acordo com uma sequência $y(t)$. Assim, a forma do sinal sísmico gerado $\psi(t)$ pode ser escrita como:

$$
\psi(t)=y(t) * w_{s}(t)
$$

Fazendo a convolução deste sinal de longa duração $\psi(t)$ com a resposta impulsiva da Terra, obtêm-se o sismograma codificado $s_{c}(t)$ :

$$
s_{c}(t)=y(t) * w_{s}(t) * r(t)+n(t)
$$

Através da correlação cruzada de $s_{c}(t)$ com a sequência de impactos $\psi(t)$ (traço piloto) obtêm-se o sismograma final:

$$
s_{d}(t)=\psi(t) \otimes s_{c}(t)=\left\{y(t) * w_{s}(t)\right\} \otimes\left\{y(t) * w_{s}(t) * r(t)+n(t)\right\}
$$

Assumindo que a sequência de impulsos é aleatória o sismograma decodificado $s_{d}(t)$ pode ser considerado aproximadamente igual a:

$$
s_{d}(t)=w_{a}(t) *(t)+n(t)
$$

onde $w_{a}(t)$ é a autocorrelação do pulso gerado no impacto. Com isto pode ser observado que a autocorrelação do traço $\psi(t)$ gera uma estimativa da wavelet $w_{a}(t)$.

\subsection{Processamento}

Levando em consideração o processamento de reflexões PS. A Figura 59 apresenta um possível fluxo de processamento paras as reflexões PS. A ordem do processamento e também a presença dos processos pode ser alterada de acordo com a situação geológica e qualidade dos dados.

As reflexões PS são observadas primordialmente nos sismogramas adquiridos com geofones de componente radial e uma Terra isotrópica com camadas planas apresenta simetria radial em relação a fonte. Isto faz com que seja necessário considerar a posição da fonte em relação aos geofones. A Figura 60 mostra um conjunto de tiro sintético de componente radial com a fonte posicionada no centro do arranjo. Isto faz com que os geofones posicionados antes da fonte possuam polaridade negativa e os geofones após a fonte possuam polaridade positiva. Desta forma, esta correção deve ser realizada em traços 
Figura 59 - Possível fluxo de processamento para a reflexão PS.

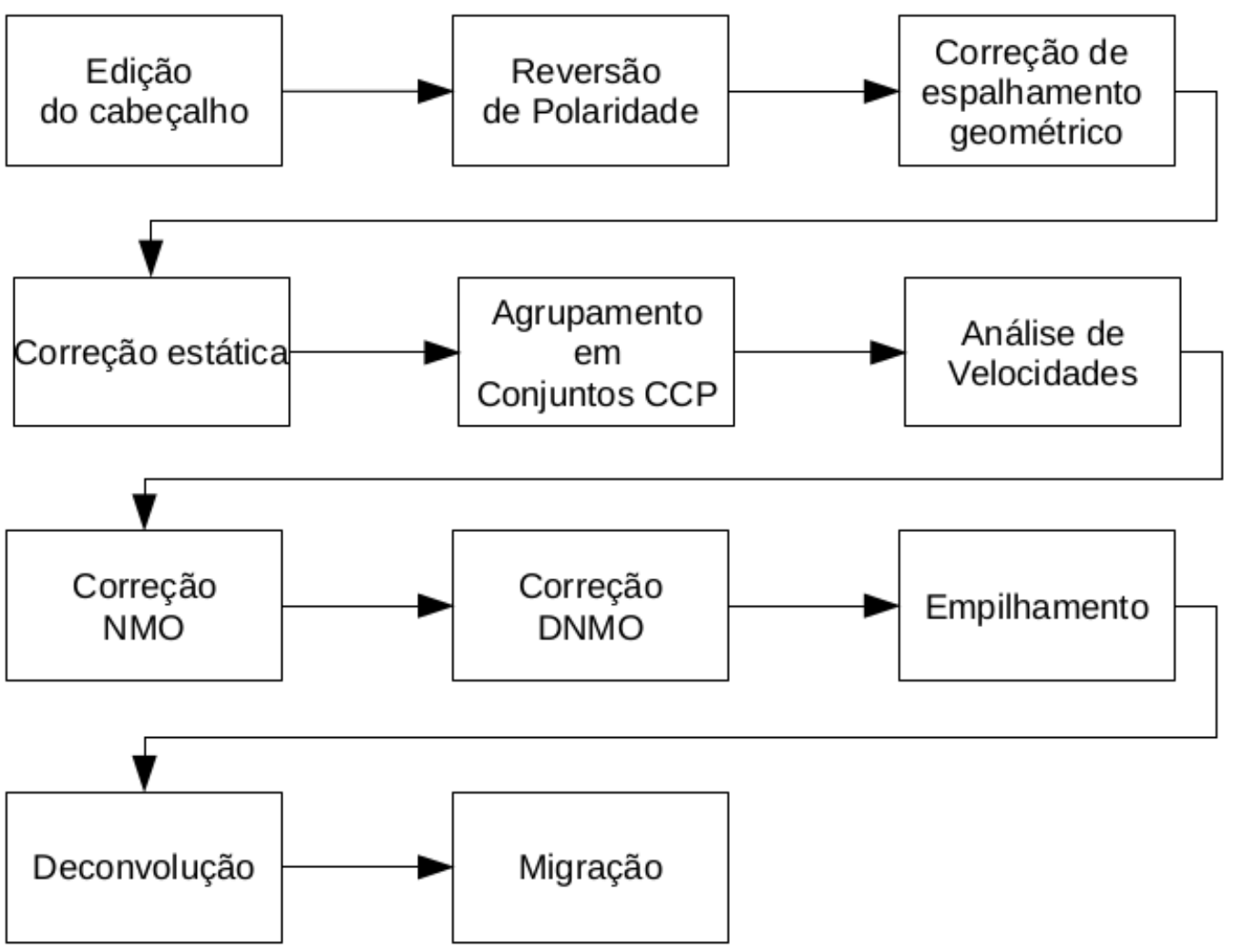

adquiridos com arranjos de lanço bi-partido ou em aquisições onde a fonte caminhe em relação aos geofones entrando no arranjo.

A correção estática para eventos PS, quando necessária, apresenta maior complexidade em relação a correção para eventos PP. A dificuldade ocorre na determinação da estática da onda $\mathrm{S}$ nos receptores.

A seguir são detalhadas as particularidades do processamento PS relacionadas às etapas de determinação dos pontos CCP, análise de velocidades, correção NMO e DMO e migração. 
Figura 60 - Conjunto de tiro de componente radial com tiro realizado no centro do arranjo.

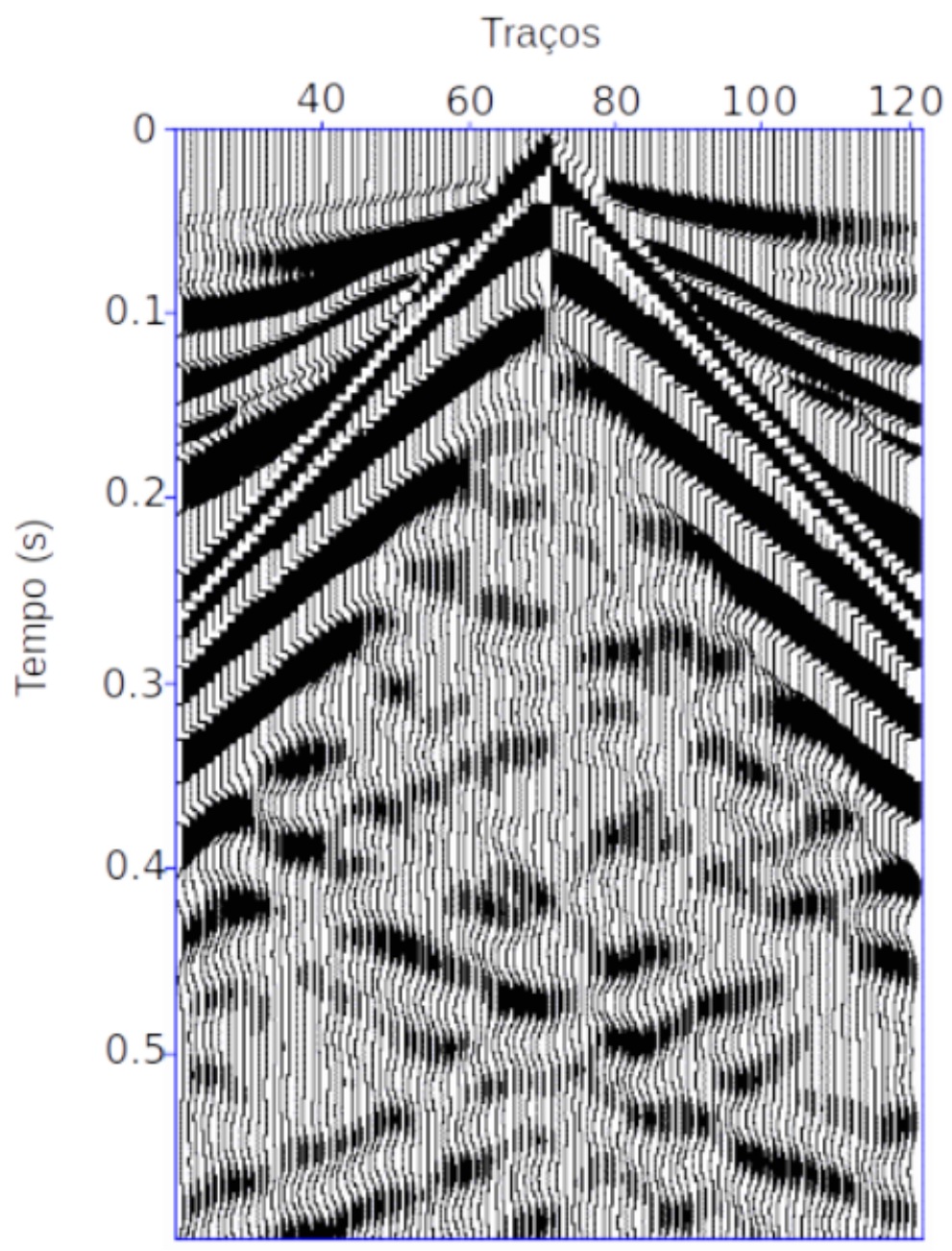

\subsubsection{Organiação em conjuntos CCP}

Uma etapa importante do processamento que influencia a aquisição é a organização dos traços sísmicos em conjuntos de traços que possuem informação sobre uma mesma região em subsuperfície. Para as reflexões PP são utilizados conjuntos de ponto médio comum (CMP) e para as reflexões PS conjuntos de ponto de conversão comum (CCP). A distinção entre ponto CMP e ponto CCP pode ser observada na Figura 61.

Os conjuntos CMP e CCP apresentam traços com diferentes afastamentos em relação a fonte onde os eventos de reflexão podem ser observados. Os eventos de reflexão presentes nestes conjuntos são utilizados na análise de velocidades e após a determinação da velocidade são corrigidos do efeito de sobretempo normal (NMO). Com isto, pode ser realizado o empilhamento dos traços levando cada conjunto a um único traço onde os eventos de reflexão presentes devem representar as interfaces geológicas entre as camadas. Desta forma, a quantidade de traços em cada conjunto (cobertura ou multiplicidade) influencia na qualidade dos dados para os procedimentos de análise de velocidades, correção NMO e 


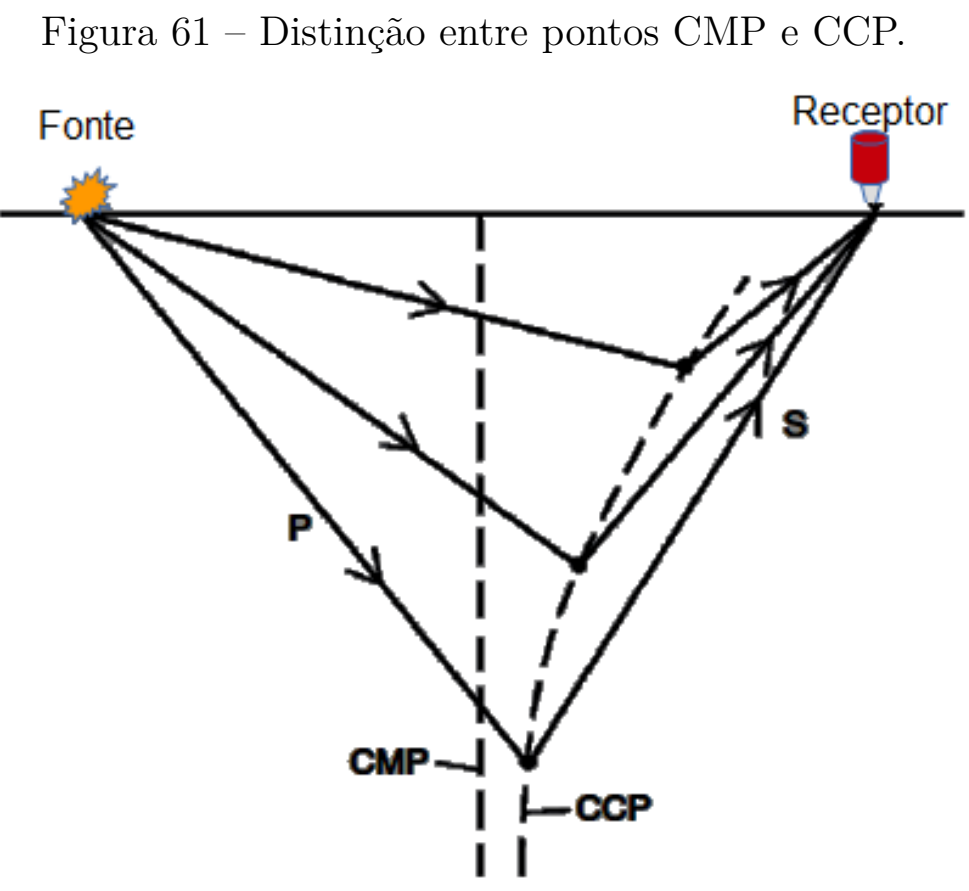

Fonte: Hardage et al. (2011).

empilhamento. A cobertura em levantamentos CMP é dada pela relação:

$$
C_{P-P}=\frac{n \Delta r}{2 \Delta s}
$$

onde $n$ é o número de canais, $\Delta r$ é o intervalo entre geofones ( ou grupo de geofones) e $\Delta s$ é o espaçamento da fonte.

No caso dos conjuntos CCP se a distância fonte-receptor for pequena com relação a profundidade do refletor a equação 5.7 define o ponto de conversão em subsuperfície.

$$
x_{c 0} \approx \frac{x \gamma}{1+\gamma}
$$

onde $\gamma=V_{P} / V_{S}$.

A equação 5.7 é chamada de aproximação assintótica, isto porque representa uma aproximação assíntota à trajetória do ponto de conversão em um caso com uma única camada. A aproximação assintótica é bastante utilizada na sísmica de petróleo já que, neste caso, os refletores são suficientemente profundos. Para profundidades menores a determinação do ponto de conversão deve ser realizada com equações que levem em consideração a profundidade dos refletores.

No trabalho de Thomsen (1999) é desenvolvida a equação 5.8 através da expansão em séries de Taylor que fornece uma boa precisão para razões afastamento/profundidade de até $1 / 0,3$. 


$$
x_{c}(x,) \approx x\left[C_{0}+C_{2} \frac{(x / z)^{2}}{1+C_{3}(x / z)^{2}}\right],
$$

com

$$
\begin{gathered}
C_{0}=\frac{\gamma}{1+\gamma}, \\
C_{2}=\frac{\gamma(\gamma-1)}{2(1+\gamma)^{3}}, \\
C_{3}=\frac{C_{2}}{\left(1-C_{0}\right)},
\end{gathered}
$$

Este tipo de equação pode ser utilizada na construção de uma carta de empilhamento de um alvo específico ou também no processamento. Assim é possível a determinação da cobertura que será obtida em um levantamento CCP. Outra forma da determinação dos pontos de conversão é através de traçado de raio.

Outro ponto importante que diferencia a aquisição CMP de uma aquisição CCP é a possível necessidade da aquisição de uma segunda linha no sentido negativo. A Figura 62 exemplifica a diferença no caminho percorrido por uma reflexão PP e por uma PS quando existe diferença lateral na litologia.

Figura 62 - Caminhos percorridos por raios diretos e reversos de reflexões PP e PS em um meio com variação lateral de litologia.

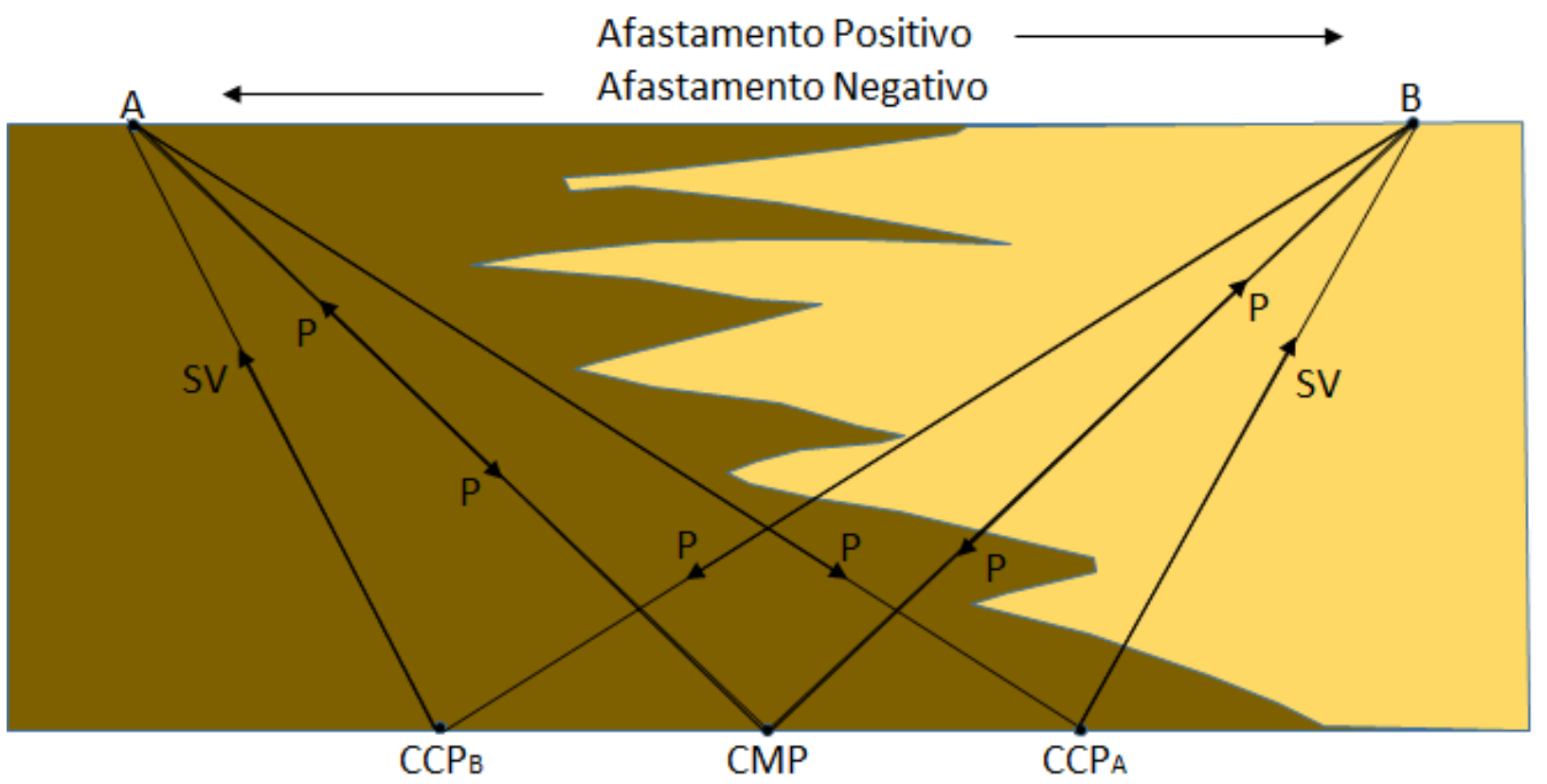

Fonte: Hardage et al. (2011). 
Além de possíveis variações de litologia a aquisição de tiros diretos e reversos torna possível a utilização da técnica desenvolvida por Grechka e Tsvankin (2002). Esta técnica utiliza eventos de reflexão PS diretos e reversos que refletiram no mesmo ponto para a reconstrução dos tempos de percurso de uma reflexão SV pura. Eliminando alguns problemas do processamento que serão vistos a seguir.

\subsubsection{Análise de velocidades}

Os diferentes métodos para análise de velocidades baseiam-se no ajuste da curva de tempo de percurso aos tempos observados nos dados sísmicos. A função de tempo de percurso das ondas sísmicas para um meio com camadas plano-horizontais homogêneas pode ser expressa em termos da distância entre fonte e receptor numa expansão em série de Taylor. Taner e Koehler (1969) desenvolveram uma expansão para as equações de tempo de trânsito e TESSMER e BEHLE (1988) desenvolveram uma especificamente para o caso de ondas convertidas. A equação da hipérbole representa um truncamento no segundo termo da série desenvolvida por Taner e Koehler (1969). Para razões afastamento/profundidade altas a equação da hipérbole não é válida para eventos de reflexão PP a partir da segunda camada e desde o início para os eventos PS. Isto torna necessária a utilização de uma aproximação melhor.

Em ??) foi avaliada a adequação de uma série de equações de tempos de trânsito para a situação de sísmica rasa, onde foi obtido melhor desempenho com a equação de hipérbole deslocada:

$$
t(x)=\left(1-\frac{1}{S}\right) t_{0}+\frac{1}{S} \sqrt{t_{0}^{2}+S \frac{x^{2}}{V_{r m s}^{2}}} .
$$

Na equação $5.12 t$ é o tempo de percurso até o refletor e de volta para o receptor, $x$ representa o afastamento entre a fonte e os receptores, $t_{0}$ é o tempo duplo de percurso para o afastamento nulo, S é o chamado de coeficiente de heterogeneidade e $V_{r m s}$ a velocidade de root mean square (RMS). Os parâmetros $S$ e $V_{r m s}$ são descritos pelas equações a seguir:

$$
\begin{gathered}
S=\frac{\mu_{4}}{\mu_{2}^{2}}, \\
V_{r m s}=\sqrt{\mu_{2}},
\end{gathered}
$$

onde

$$
\mu_{j}=\frac{\sum_{k=1}^{n} t_{k} V_{k}^{j}}{\sum_{k=1}^{n} t_{k}}
$$


e $V_{k}$ é a velocidade intervalar da camada $k$ e $t_{k}$ é o tempo de percurso na camada $k$ normal à interface (vertical se a camada for plana).

A equação de hipérbole deslocada 5.12 foi desenvolvida por Malovichko (1978) e estudada em detalhe no trabalho de Castle (1994). Neste último a equação de hipérbole deslocada foi adotada como uma forma geral de uma equação de NMO onde o parâmetro $\mathrm{S}$ controla o desvio da hiperbolicidade para afastamentos mais longos.

A presença de um terceiro termo torna a estimativa da velocidade mais difícil. Algumas técnicas alternativas para a análise de velocidades são descritas em MoldoveanuConstantinescu e Sacchi (2005) e Watanabe (2010).

A velocidade obtida pela equação 5.12 é uma velocidade RMS da reflexão PS. Para a estimativa direta da velocidade da onda S em Grechka e Tsvankin (2002) foi desenvolvida uma técnica para a reconstrução dos tempos de percurso de uma de uma reflexão SS a partir de tiros diretos e reversos PS. Conforme a Figura 63 esta reconstrução pode ser realizada em meios homogêneos para eventos incidentes em um mesmo ponto.

Figura 63 - Tiro direto e tiro reverso de reflexão PS incidindo em um mesmo ponto.

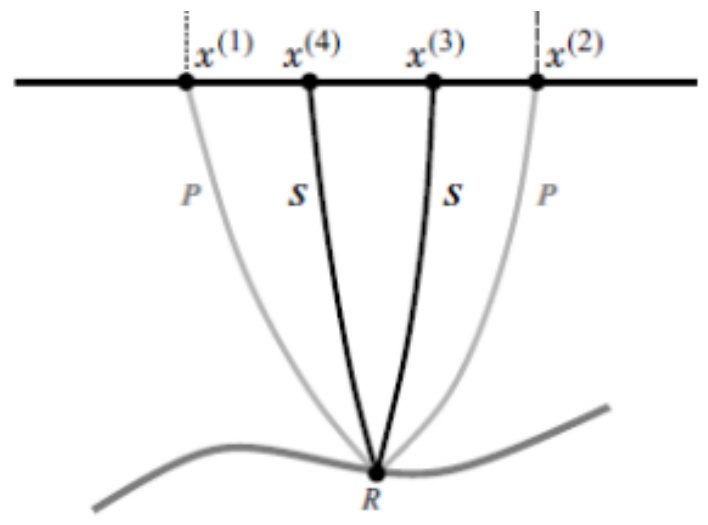

Fonte: Grechka e Tsvankin (2002).

Para a situação da Figura 63 a reconstrução do tempo de reflexão SS pode ser escrito como:

$$
t_{s s}\left(x^{(3)}, x^{(4)}\right)=t_{p s}\left(x^{(1)}, x^{(3)}\right)+t_{p s}\left(x^{(2)}, x^{(4)}\right)-t_{p p}\left(x^{(1)}, x^{(2)}\right) .
$$

Em Stovas e Ursin (2007) foi mostrado que para um conjunto de camadas planas e homogêneas com velocidade constante, não há assimetria no tempo de percurso PS entre os tiros diretos e reversos. Isto permite reescrever a equação anterior na forma simplificada:

$$
t_{s s, 0}=2 t_{p s, 0}-t_{p p, 0}
$$

e escrever

$$
t_{s s, 0} v_{s s}^{2}=2 t_{p s, 0} v_{p s}^{2}-t_{p p, 0} v_{p p}^{2}
$$


onde o índice 0 serve para indicar o tempo no afastamento nulo.

Estas equações tornam desnecessária a utilização de tiros diretos e reversos e tornam possível o cálculo da velocidade da onda $\mathrm{S}$ através da estimativa da velocidade da reflexão PS, se as camadas forem planas.

\subsubsection{Correção de sobretempo normal (NMO)}

Em um evento de reflexão observado em um afastamento x o sobretempo normal é a diferença entre o tempo observado nesta distância e o tempo de reflexão observado no afastamento nulo:

$$
\Delta T_{N M O}=t_{x}-t_{0}
$$

Deve ser observado que em 5.19 o tempo $t_{0}$ é obtido por uma equação de tempo de percurso. No caso das reflexões PP com afastamentos dos receptores longos em relação a profundidade dos refletores ou reflexões PS a aproximação a ser utilizada também deve ser mais precisa do que a hiperbólica como a fornecida pela equação 5.12.

A correção (DMO) é aplicada quando existem refletores com mergulho. Neste caso, os traços de um conjunto CMP ou CCP, não envolvem um único ponto. O procedimento DMO corrige o espalhamento do ponto de reflexão, como exemplificado na Figura 65.

Definindo a dispersão PS como o deslocamento $(\Delta)$ dentro de um conjunto CCP do ponto de relflexão normal (afastamento nulo) na direção da fonte. Observando a Figura 66 onde uma onda $\mathrm{P}$ gerada na fonte percorre o caminho SC e uma SV convertiva percorre o caminho CR pode ser visto que $\Delta$ é dado por

$$
\Delta=\delta \cos \theta
$$

onde $\delta$ é dispersão horizontal em superfície, dado pelo deslocamento do CCP em direção à fonte. 
Figura 64 - Distribuição dos pontos de reflexão: (a) refletor plano (coincide com a projeção do ponto CMP); (b) refletor com mergulho.
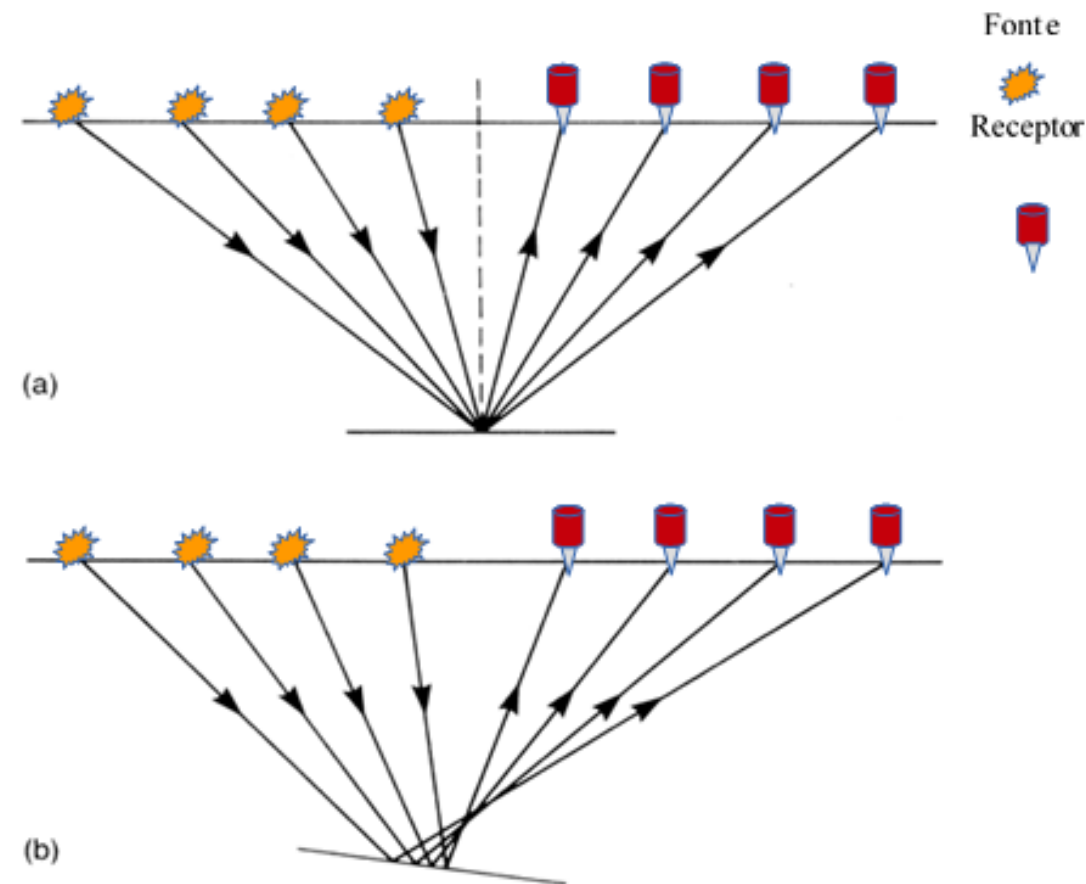

Fonte: Harrison (1992).

Figura 65 - Geometria do caminho do raio para uma reflexão PS em um refletor com mergulho.

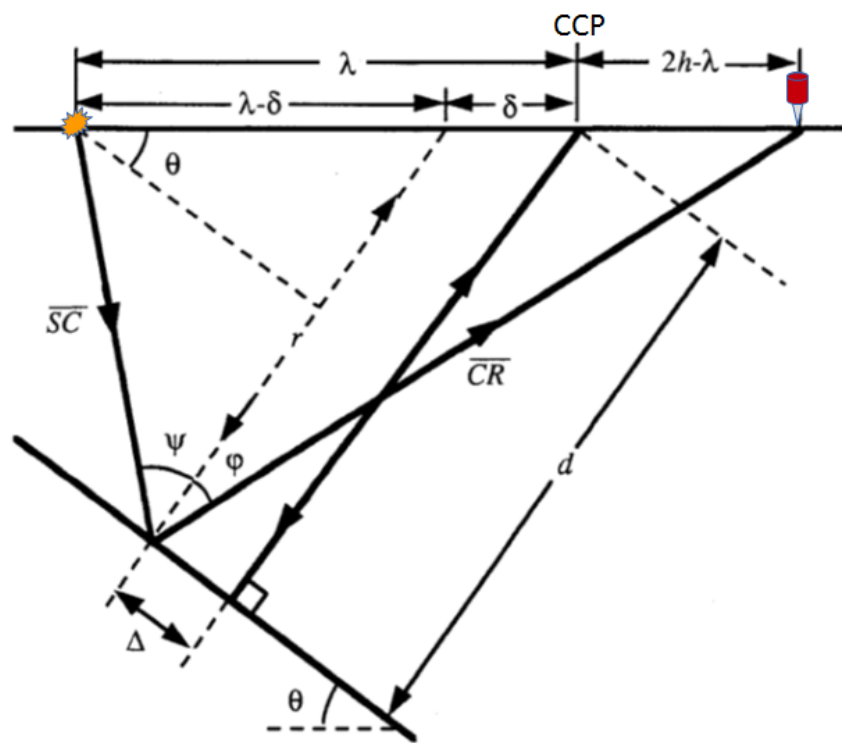

Fonte: Harrison (1992).

Em Harrison (1992) foi obtida a seguinte equação para $\delta$ :

$$
\begin{aligned}
& \left(\gamma^{2}-1\right)[(\lambda-\delta)-4 h] \cos ^{2} \theta(\lambda-\delta)^{3}+ \\
& \left\{\gamma^{2}(d-\lambda \operatorname{sen} \theta)^{2}+\left(\gamma^{2}-1\right) 4 h^{2} \cos ^{2} \theta-[d+(2 h-\lambda) \operatorname{sen} \theta]^{2}\right\}(\lambda-\delta)^{2} \\
& -4 h \gamma^{2}(d-\lambda \operatorname{sen} \theta)^{2}(\lambda-\delta)+4 h^{2} \gamma^{2}(d-\lambda \operatorname{sen} \theta)^{2}=0
\end{aligned}
$$


Considerando uma situação de refletores profundos em relação ao afastamento, a equação 5.21 pode ser aproximada para a obtenção de $\Delta$, que substituída na equação 5.20 fica:

$$
\Delta_{p s} \approx \frac{4 \gamma}{(\gamma+1)^{2}}\left[1+\frac{2 h(\gamma-1)}{d(\gamma+1)} \operatorname{sen} \theta\right]
$$

A equação anterior mostra que mesmo para refletores planos $(\theta=0)$ ocorre dispersão do ponto de reflexão PS. A Figura66 mostra novamente uma onda P incidente sendo convertida em um refletor com mergulho. Porém, desta vez a origem está deslocada para o ponto médio. Com base na Figura 66 pode ser escrita a equação 5.23 que permite a aplicação de uma correção DMO para reflexões PS corrigidas de NMO.

Figura 66 - Geometria do caminho do raio para uma reflexão PS em um refletor com mergulho com origem deslocada para o ponto médio.

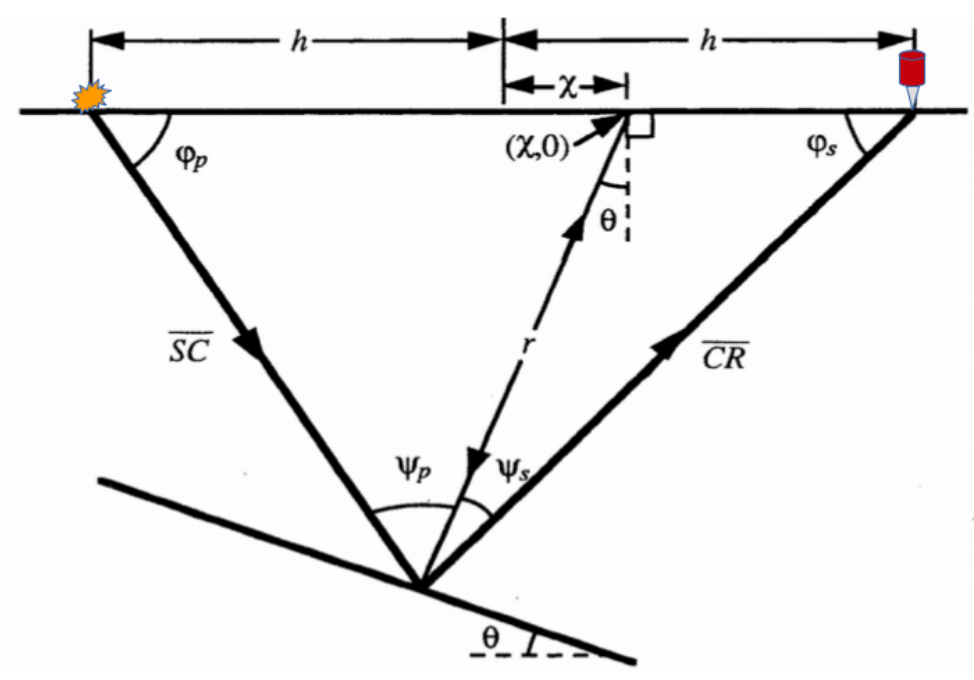

Fonte: Harrison (1992).

$\tau^{2}=\frac{\left(h^{2}-\chi^{2}\right)}{V_{P}^{2}(h-\chi)+V_{S}^{2}(h+\chi)}\left\{\frac{V_{P}^{2}\left(h-\xi_{0}\right)+V_{S}^{2}\left(h+\chi_{0}\right)}{h^{2}-\chi_{0}^{2}} t_{n}^{2}+\left(\chi-\chi_{0}\right)\left(V_{P}^{2}-V_{S}^{2}\right)\left(\frac{V_{P}+V_{S}}{V_{P} V_{S}}\right)^{2}\right\}$

$\mathrm{Na}$ equação 5.23 é qualquer afastamento a partir do ponto médio, $\chi_{0}$ é o ponto de conversão no mergulho nulo, $t_{n}$ é o tempo corrigido de NMO.

\subsubsection{Migração pós-empilhamento}

A migração de dados PS empilhados pode ser realizada como uma soma ao longo de uma trajetória de tempo de percurso. Esta é associada com um par fonte-receptor 
coincidente na superfície e um ponto de difração na subsuperfície. Esta descrição é a mesma que seria dada para uma migração de um dado PP. A diferença entre a migração PP e PS está na velocidade da trajetória de soma.

Em Yilmaz (2001) a equação 5.24 é utilizada para o cálculo do tempo de percurso no afastamento nulo PS utilizada na trajetória de soma da difração.

$$
t^{2}=t_{0}^{2}+\left(\frac{\gamma+1}{V_{P}}\right)^{2} x_{P}^{2}
$$

onde $\gamma=V_{P} / V_{S}, x_{P}$ o ponto de conversão e $t_{0}=\left(\frac{1}{V_{P}}+\frac{1}{V_{S}}\right)^{2}$.

A equação 5.24 possui a mesma forma das equações utilizadas para a trajetória de soma de difração em um dado PP. Entretanto, a velocidade de migração é dado por $V_{P} /(\gamma+1)$. Uma vez estimada esta velocidade pode ser utilizada em conjunto com os algoritmos convencionalmente utilizados em dados PP. 


\section{Dados do Terreno do Instituto de Física da USP}

No terreno do Instituto de Física da USP foram adquiridos dados de componente vertical e radial para um levantamento de reflexão segundo a técnica CMP. A região estudada pertence ao limite leste da Bacia Sedimentar de São Paulo. Esta é caracterizada por possuir sedimentos argilosos, arenosos e siltosos, no sentido superfície-embasamento. Uma descrição completa da geologia da Bacia Sedimentar de São Paulo pode ser encontrada em Riccomini (1989).

A linha de aquisição foi locada próxima a poços de investigação geológica da região (Figura 67). Na aquisição de dados foi utilizado como fonte de energia sísmica um compactador de solos com a técnica Mini-Sosie(BARBIER, 1983). Para o registro dos conjuntos de tiro foram utilizados 48 geofones de $40 \mathrm{~Hz}$ para a componente vertical e 47 geofones de $28 \mathrm{~Hz}$ para a radial por conjunto de tiro. Os geofones utilizados no registro da componente vertical foram dispostos em uma linha paralela aos geofones de componente radial.

Figura 67 - Mapa de localização da área estudada e perfil sísmico.

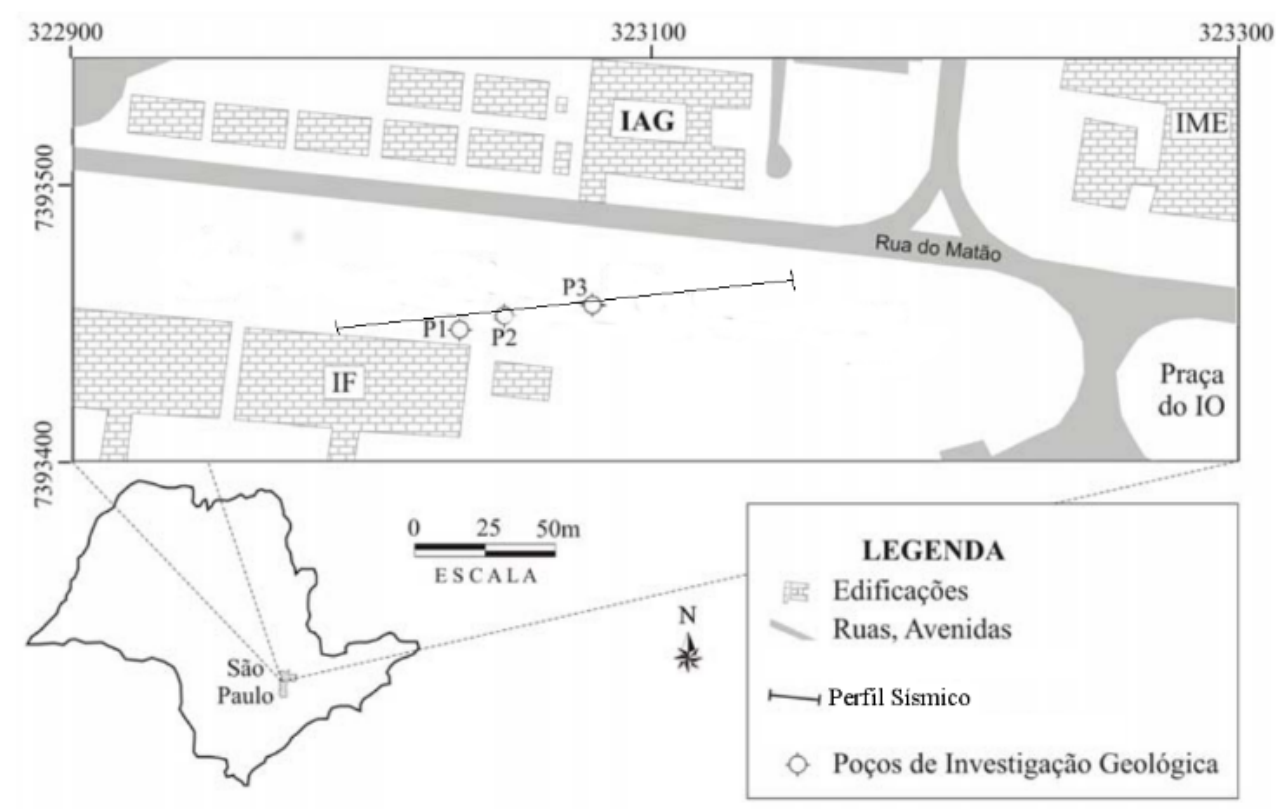

Fonte: Porsani et al. (2004)

Além das informações litológicas dos poços (Figura 68) em Ullah, Prado e Lisa (2017) foi obtido um modelo através de um ensaio downhole. Este modelo pode ser obser- 
Tabela 2 - Perfil de velocidades extraído do poço P2. Extraído de Ullah, Prado e Lisa (2017).

\begin{tabular}{ccccc}
\hline Camada & $V_{P}(\mathrm{~m} / \mathrm{s})$ & $V_{S}(\mathrm{~m} / \mathrm{s})$ & $\rho\left(\mathrm{kg} / \mathrm{m}^{3}\right)$ & Profundidade $(\mathrm{m})$ \\
\hline 1 & 1000 & 230 & 1700 & - \\
2 & 1400 & 350 & 1700 & 10 \\
3 & 2000 & 430 & 1700 & 28 \\
4 & 2200 & 560 & 1700 & 38 \\
5 & 3500 & 1300 & 1900 & 55 \\
\hline
\end{tabular}

vado na Figura 69 e Tabela 2 e foi atribuído ao poço P2 (Figura 68).

Figura 68 - Correlação entre os perfis geológicos dos poços do sítio do Instituto de Física.

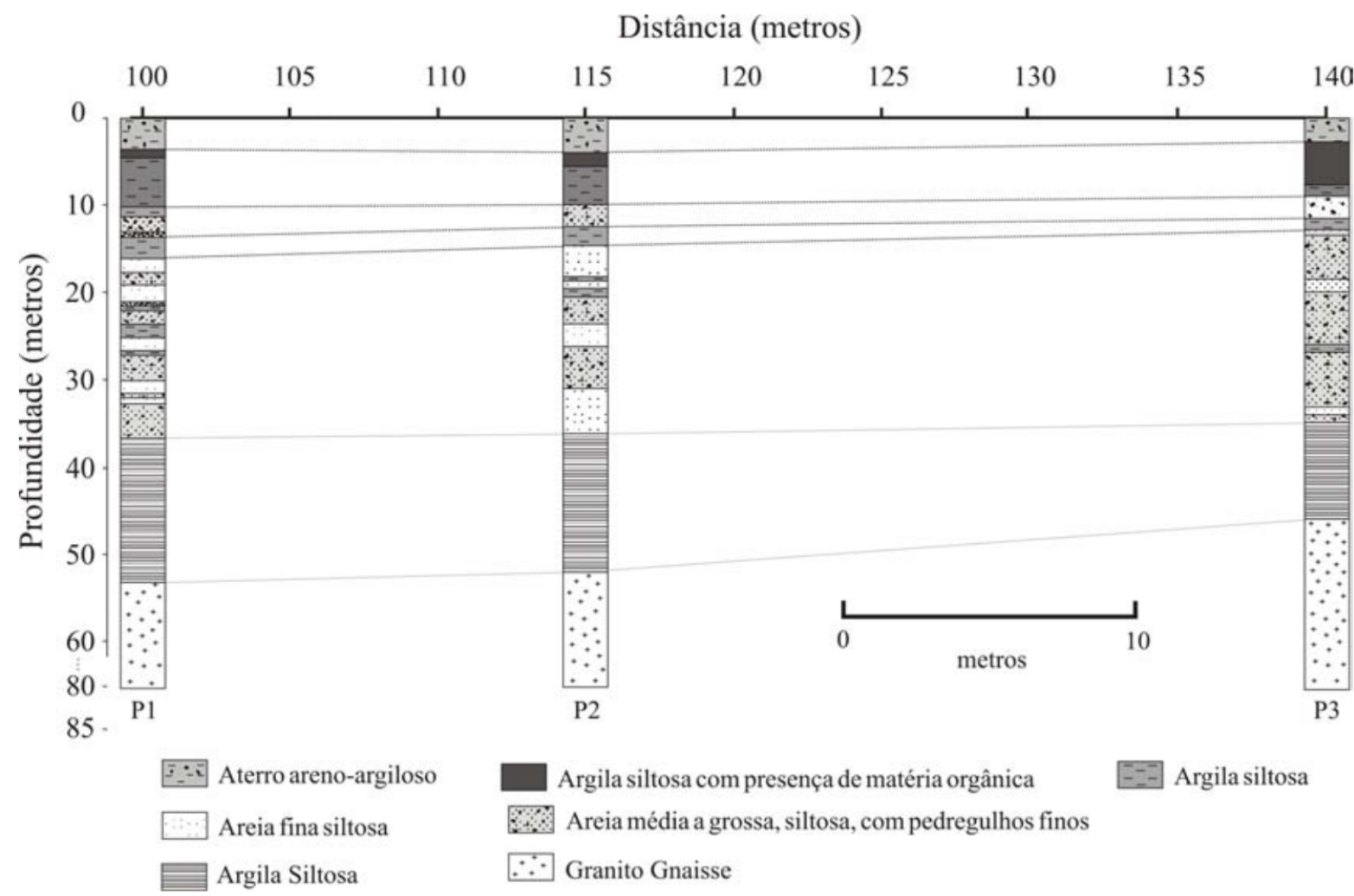

Fonte: Porsani et al. (2004)

Antes da aquisição CMP de reflexão foram realizados ensaios de análise de ruído com um tiro direto e um reverso, também utilizando a técnica Mini-Sosie como fonte, e empregando geofones verticais e horizontais, Figuras 70 e 71, respectivamente. Estes ensaios foram importantes para definir a geometria de aquisição do levantamento CMP, visando o imageamento do embasamento; e para fornecer informações que possibilitaram estimar os parâmetros da parte mais rasa do sítio.

A aquisição do levantamento de reflexão foi realizada mantendo o arranjo de geofones fixo e a fonte foi movimentada no sentido do arranjo, a intervalos iguais ao espaçamento 
Figura 69 - Perfil de velocidade para Vp e Vs para o poço P2.

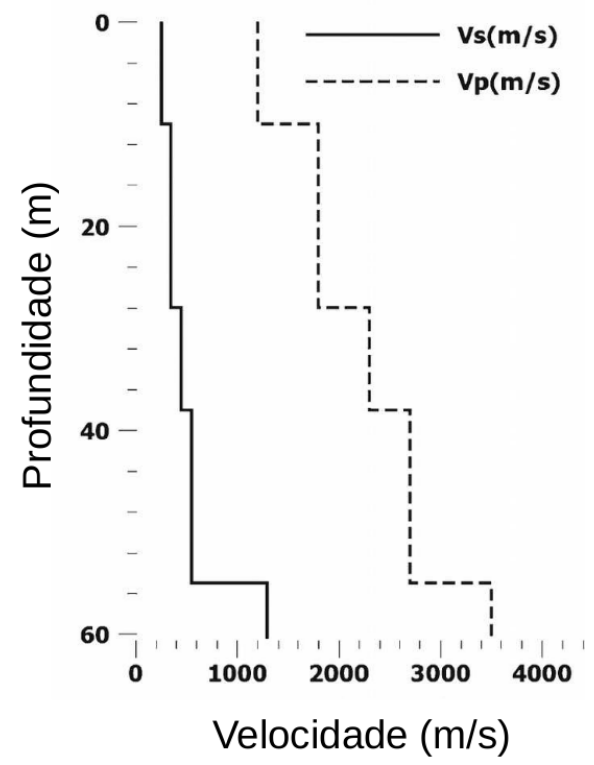

Fonte: Ullah, Prado e Lisa (2017)

entre geofones. Após a execução de um número de tiros igual à multiplicidade desejada, uma quantidade de geofones igual a esta multiplicidade é movida para o final do arranjo. Assim, a97 aquisição dos conjuntos de tiros é reiniciada para uma nova base de geofones fixos. Esta técnica de aquisição também foi aplicada em Diogo, Le Diagon e Prado (2004).

Nesta aquisição foram realizados 48 pontos de tiro no total. A movimentação dos geofones ocorreu após realizados os primeiros 24 tiros. O tempo de geração do sinal pela fonte foi de 31 segundos, o intervalo de amostragem foi de $500 \mu s$ e a janela temporal final de $1000 \mathrm{~ms}$, esta foi posteriormente cortada para $600 \mathrm{~ms}$. A cobertura máxima foi de $2400 \%$ entre os CMPs 24 e 96, conforme a carta de empilhamento da Figura 72. 
Figura 70 - Conjuntos de tiro adquiridos com geofones de componente vertical.

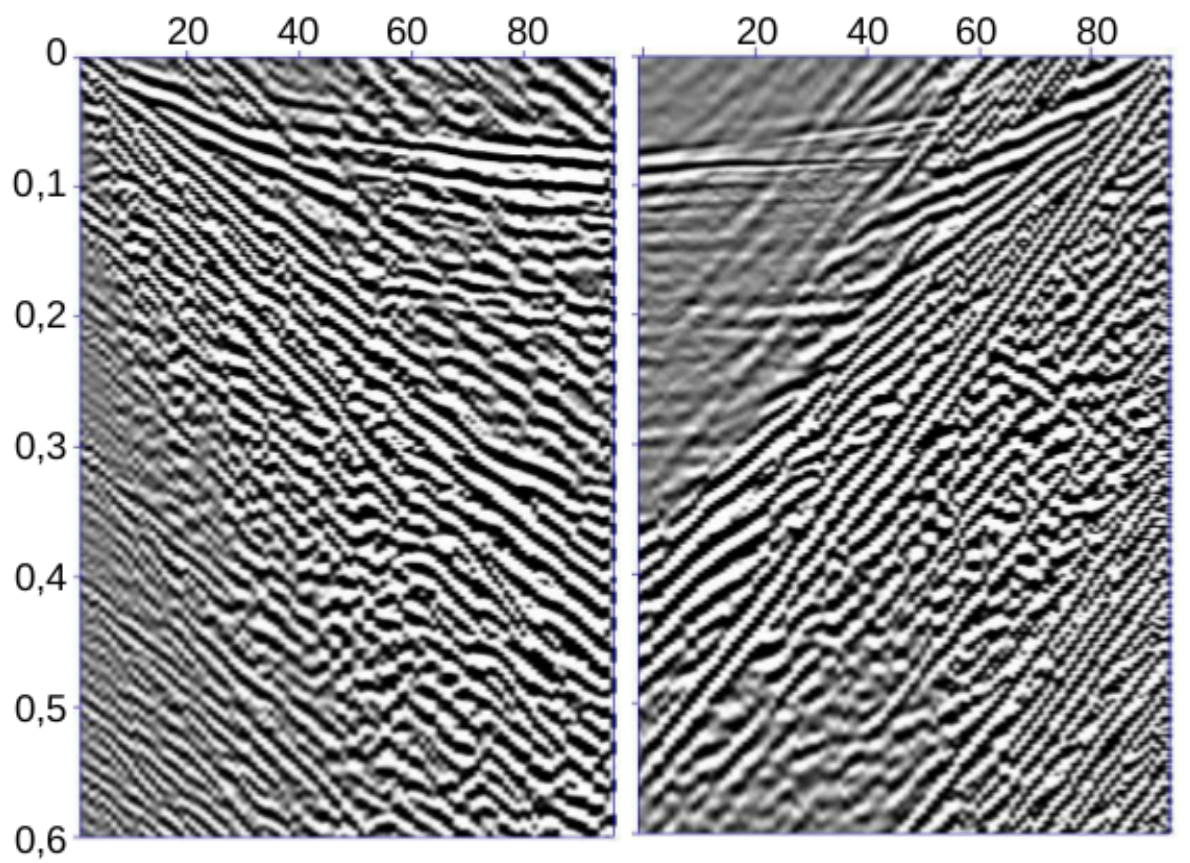

Figura 71 - Conjuntos de tiro adquiridos com geofones de componente radial.

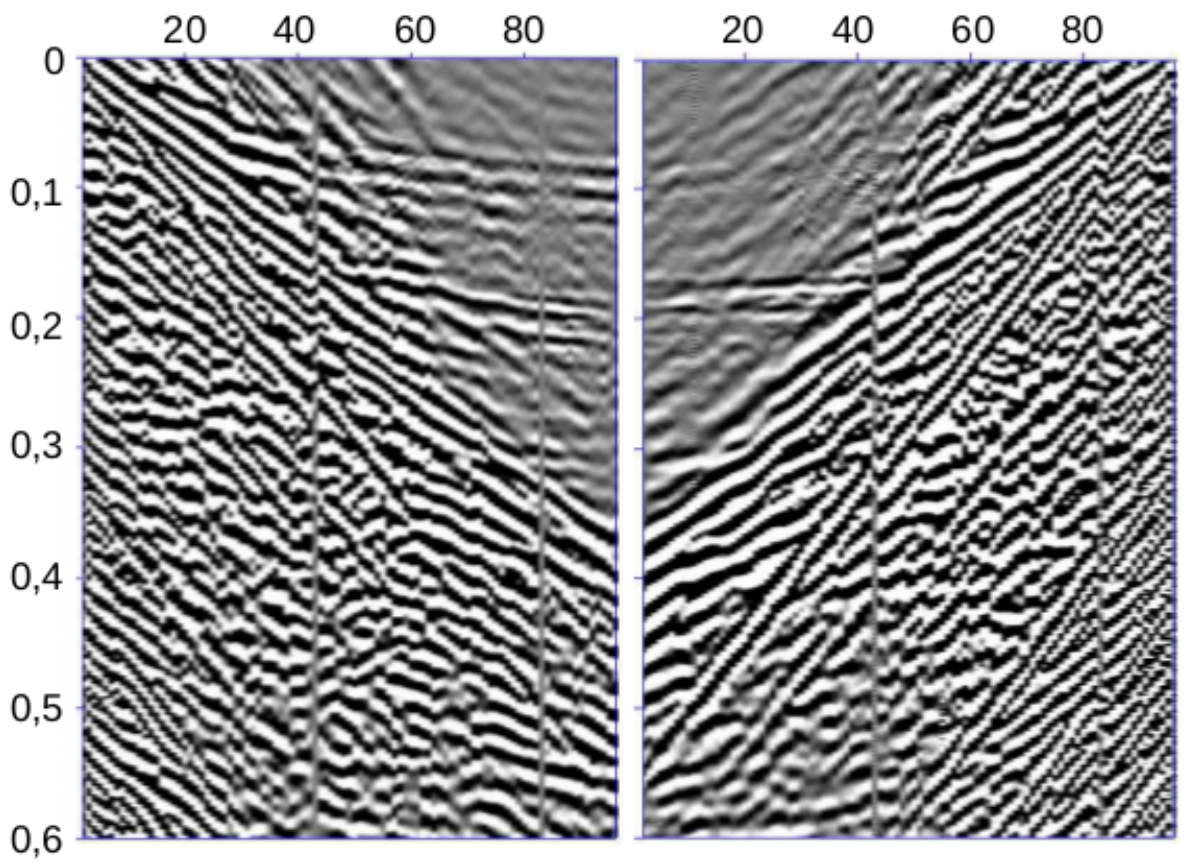


Figura 72 - Carta de empilhamento para a aquisição CMP da componente vertical.

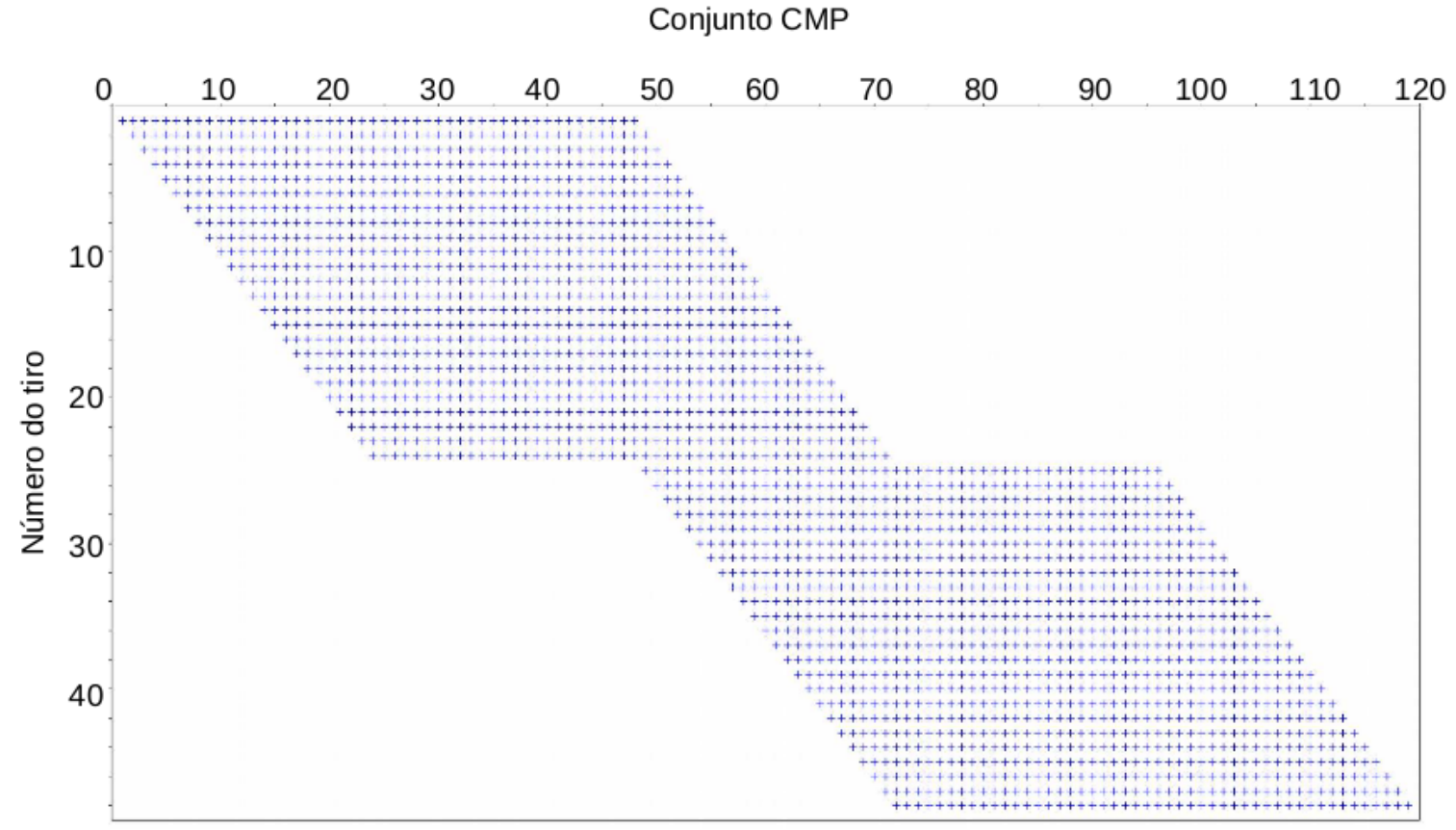




\subsection{Processamento de refração}

As primeiras chegadas observadas nos dados de análise de ruído e nos dados adquiridos para o levantamento de reflexão foram utilizadas em conjunto para a obtenção das velocidades da onda $\mathrm{P}$ na parte mais rasa do modelo. Foi utilizado o método de tomografia de refração através do software SeisImager ((GEOMETRICS, )). Os resultados obtidos são coerentes com as velocidades da Tabela 2. Entretanto, neste modelo são observadas velocidades mais baixas nas partes rasas do modelo.

Figura 73 - Modelo de velocidades Vp obtido a partir das primeiras chegadas observadas nos tiros de análise de ruído e nos conjuntos de tiro do levantamento de reflexão.



\subsection{Processamento de ondas superficiais}

Para a estimativa da velocidade das ondas S na parte superficial do modelo foi utilizado o software Geopsy. Este faz uso do algoritmo de inversão da curva de dispersão proposto em Wathelet (2008). Neste caso foram utilizados os conjuntos de tiro adquiridos para análise de ruído.

A parte central dos conjuntos de tiro de análise de ruído é coincidente. Os resultados mostrados na Figura 75 são coerente com os apresentados na 2 apenas com divergência para a última camada considerada. 
Figura 74 - Ajuste das curvas de dispersão obtidas a partir dos conjuntos de tiro de análise de ruído. (a) Tiro direto; (b) Tiro reverso.

(a)

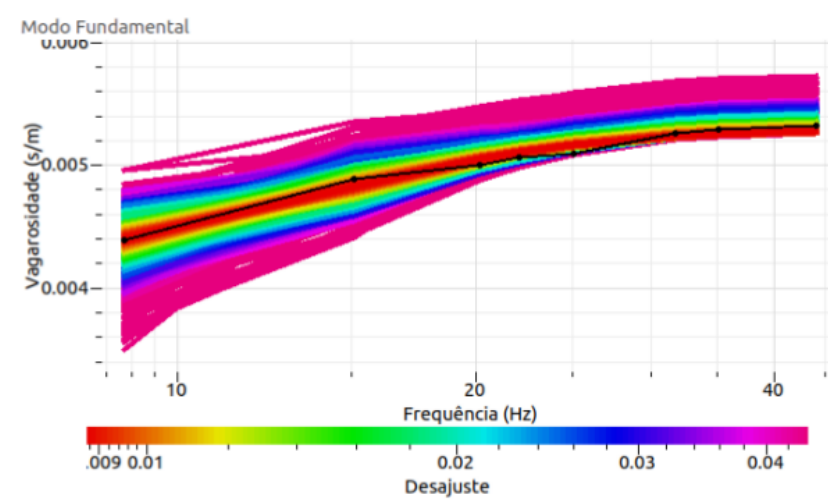

(b)

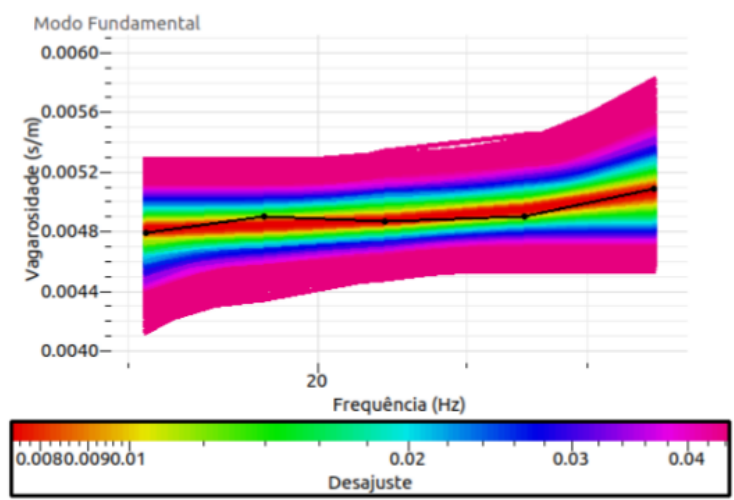

Figura 75 - Resultado da inversão das curvas de dispersão obtidas a partir conjuntos de tiro de análise de ruído. (a) Tiro direto; (b) Tiro reverso.

(a)

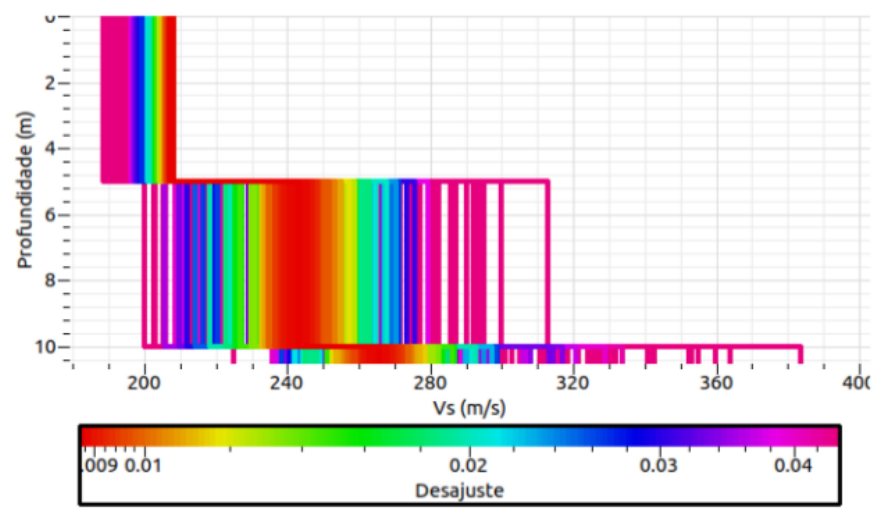

(b)

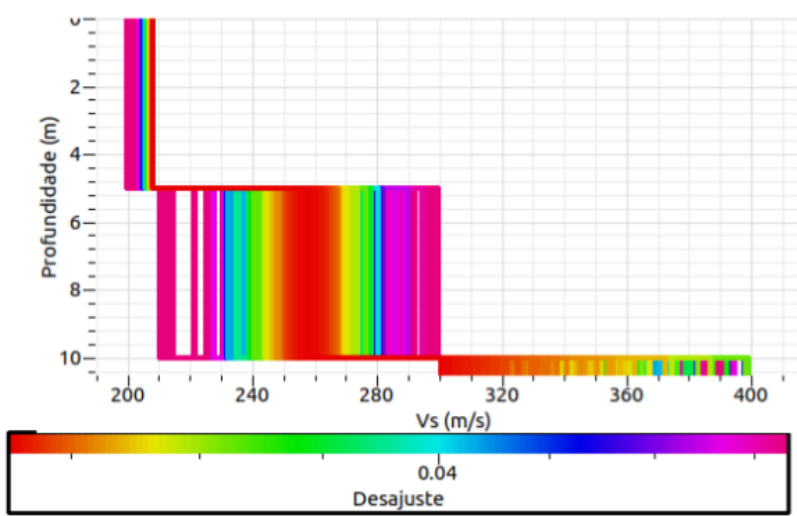

\subsection{Processamento de reflexão}

O processamento dos dados foi realizado com o pacote Seismic Unix (SU) (COHEN; STOCKWELL, 2015). Os processos de filtragem e ganho do dados de reflexão PP e PS foram os mesmos e os resultados são mostrados conjuntamente.

Para a redução de ruídos aleatórios e coerentes foram aplicados filtros passa-banda e FK, respectivamente. Para a componente vertical foi aplicado um filtro passa-banda definido pelas frequências $\mathrm{f}=(20,30,160,240) \mathrm{Hz}$ (Figura 76 ) e para a componente radial o filtro foi dado por $\mathrm{f}=(20,30,160,175) \mathrm{Hz}$ (Figura 77). Na sequência, antes da aplicação do filtro FK, foi realizado um anho de balanceamento, necessário para a visualização adequada do espectro de amplitude FK. O resultado do uso do filtro FK, em ambas as componentes são exemplificados na Figura 78. 
Figura 76 - Conjuntos de tiro de reflexão de componente vertical. (a) Dados brutos; (b) Após a aplicação do filtro passa-banda.

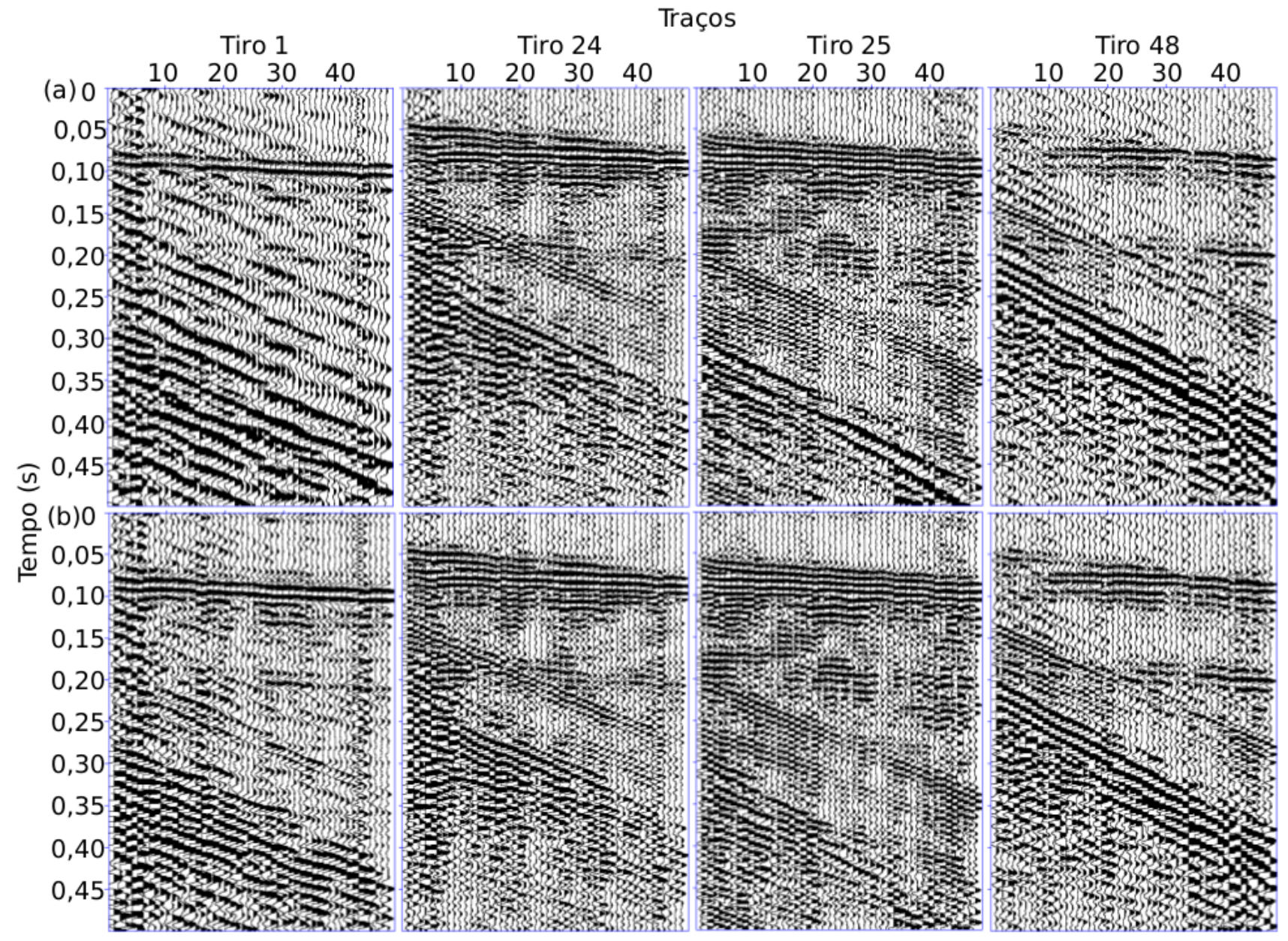


Figura 77 - Conjuntos de tiro de reflexão de componente radial. (a) Dados brutos; (b) Após a aplicação do filtro passa-banda.

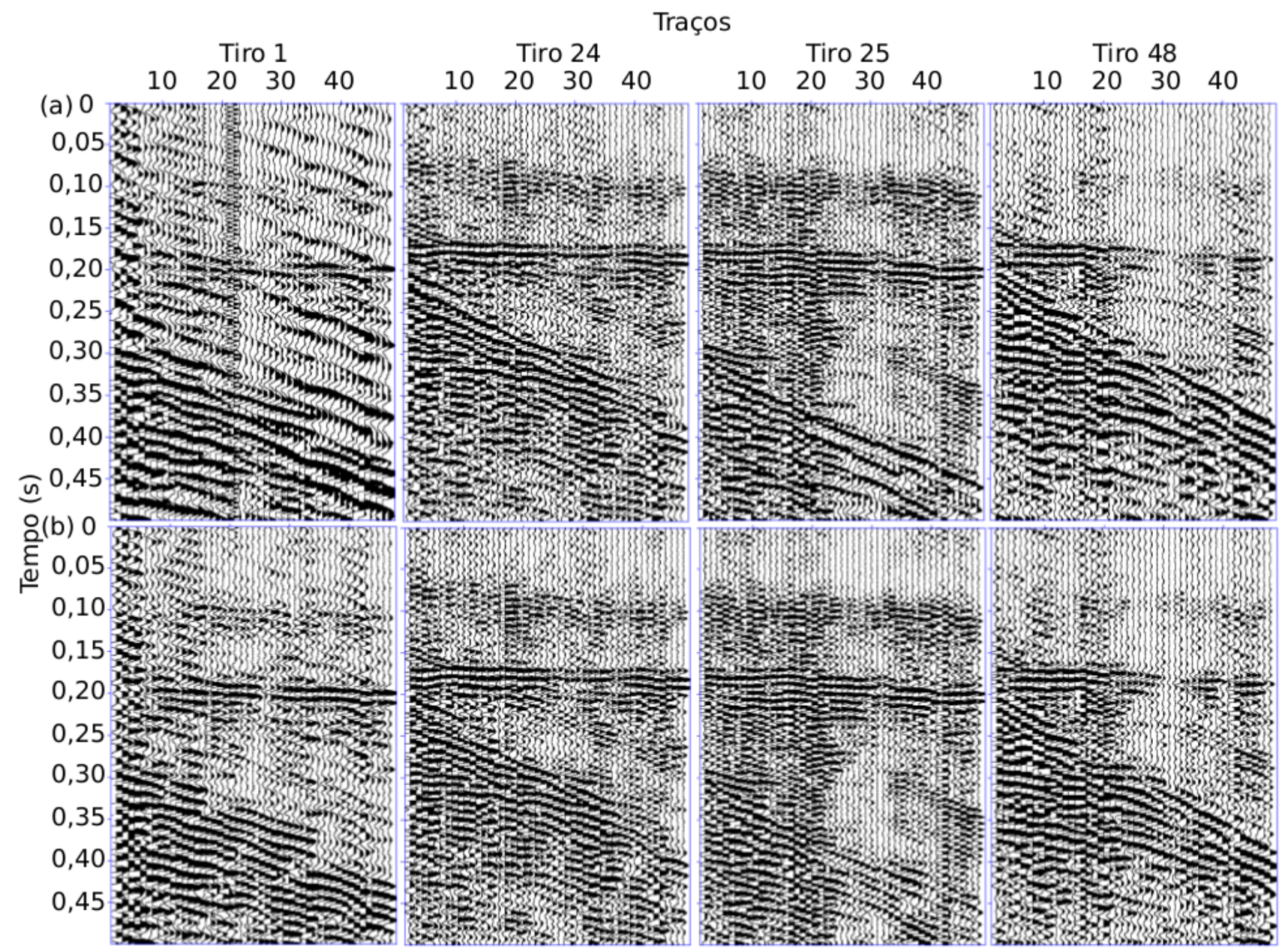


Figura 78 - Conjuntos de tiro de reflexão após a aplicação do filtro FK. (a) Componente Vertical; (b) Componente Radial.

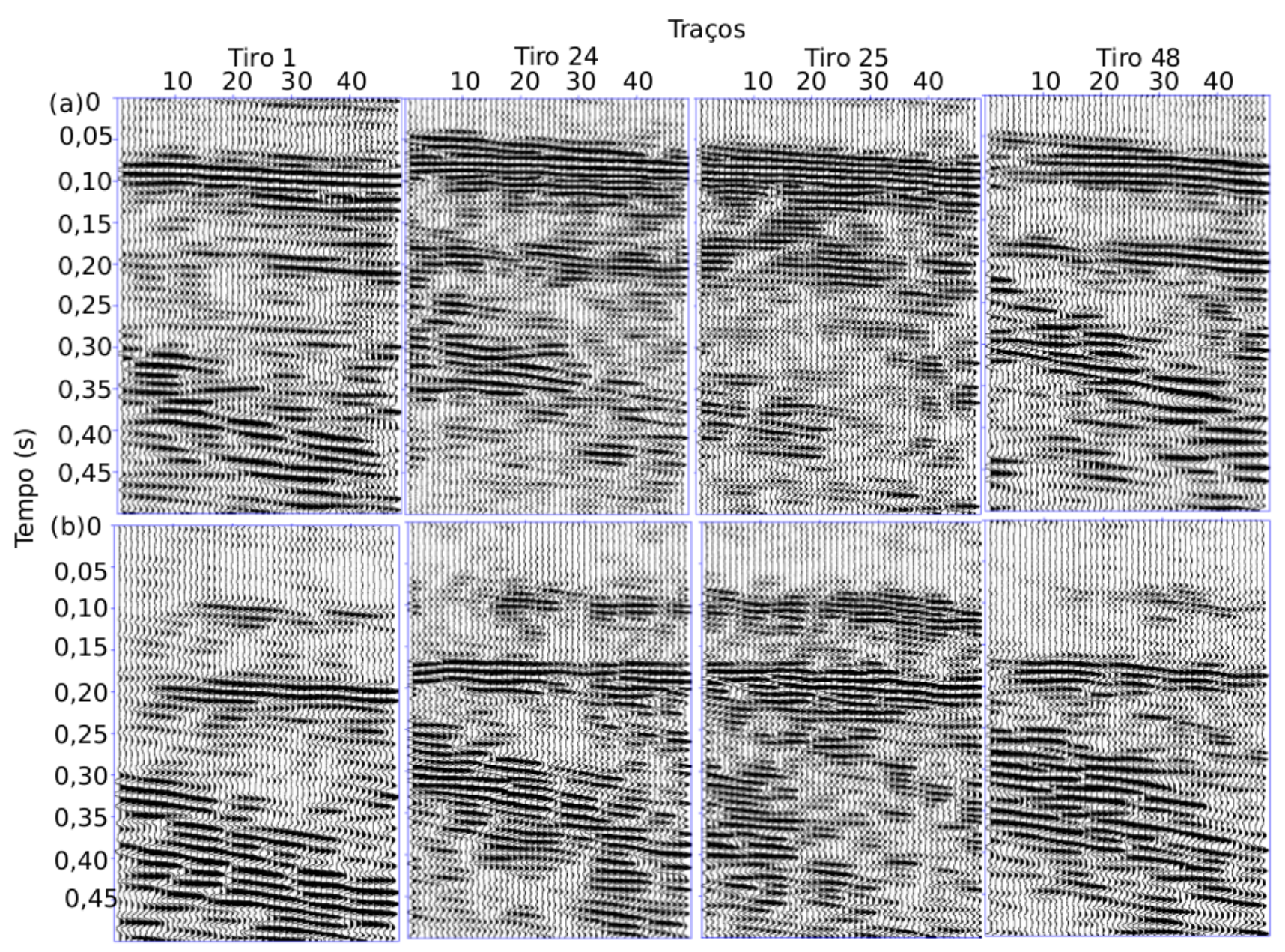




\subsubsection{Processamento de reflexões PP}

Os dados de componente vertical foram reorganizados em conjuntos CMP onde pôde ser realizada a análise de velocidades. A velocidade do evento de reflexão fica entre 1600 e 1700 m/s. Para a correção de normal moveout (NMO) foram obtidos melhores resultados com uma velocidade de 1668 m/s em toda a seção. Nas Figuras 79 e 80 são apresentadas as seções empilhadas em tempo e em profundidade, respectivamente. Para a conversão de tempo em profundidade devem ser consideradas as velocidades intervalares, cujos valores foram103 determinados combinando-se a interpretação das refrações e ondas superficiais (seções 6.1 e 6.2, respectivamente).

Figura 79 - Seção empilhada obtida a partir dos sismogramas de reflexão PP.

\section{Conjunto CMP}

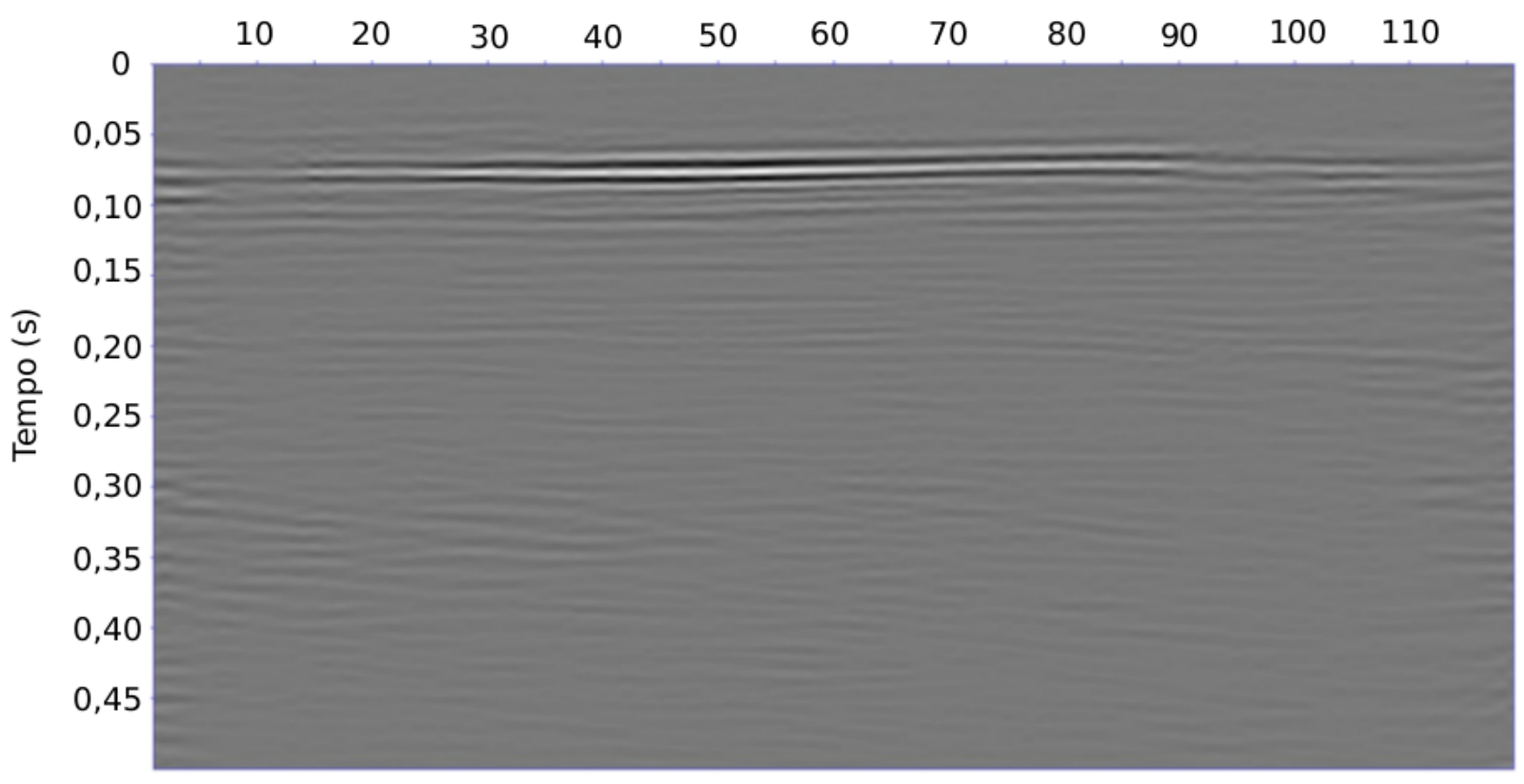

Figura 80 - Seção empilhada ( para a onda PP) em profundidade.

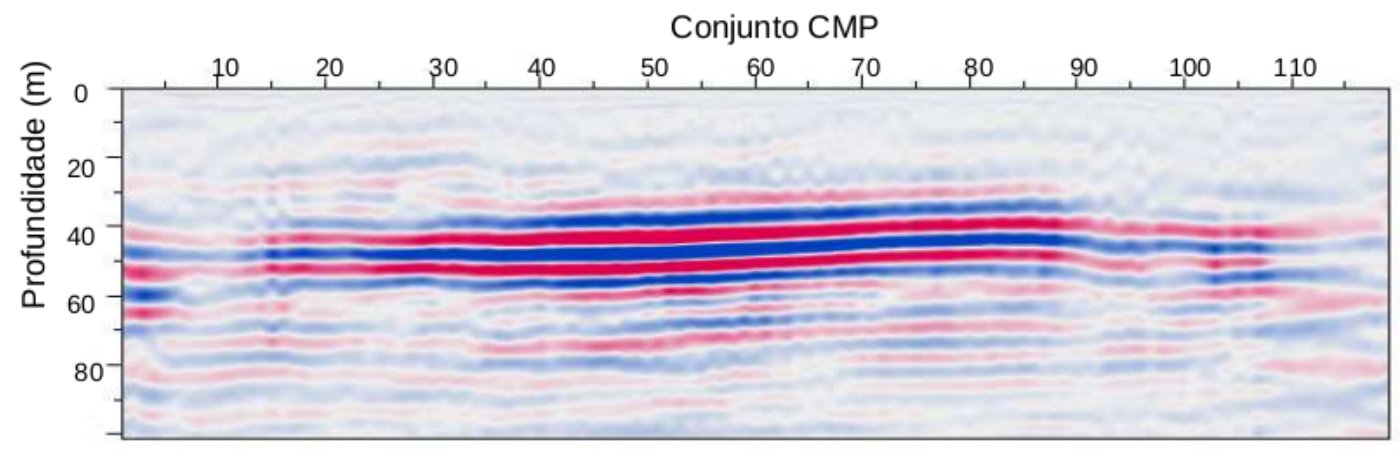




\subsubsection{Processamento de reflexões PS}

A reorganização dos dados de componente horizontal foi baseada em traçado de raio. Utilizando uma velocidade média de $1100 \mathrm{~m} / \mathrm{s}$ foram calculados os pontos de conversão para uma interface em 35 metros de profundidade, a partir da qual começam a ser observados eventos de reflexão em algumas posições da seção empilhada obtida do processamento de reflexão da onda PP (Figura 80). Isto ocorre, provavelmente, pela passagem para a camada de argila siltosa como pode ser observado nos perfis geológicos da 68.

Como a seção possui um único evento de reflexão PS, os dados foram organizados de acordo com os pontos de conversão para a interface localizada aproximadamente em 35 metros de profundidade. Assim sendo, foi realizada uma nova análise de velocidades sobre os conjuntos de números 65,75,85,95. Destes conjuntos CCP foram tomados os tempos de chegada do evento de reflexão observado. Para a estimativa da velocidade foi utilizada a equação de hipérbole deslocada assim como em (BOKHONOK, 2010). Esta aproximação foi criada por Malovichko (1978) e melhor estudada por Castle (1994).

Com isto, foi realizado empilhamento e conversão de tempo para profundidade da seção. Para conversão tempo-profundidade, além da análise de velocidades dos eventos de reflexão PS, foi utilizado o resultado obtido com o MASW. A seção empilhada obtida para as reflexões PS é coerente com a das reflexões PP. Foi utilizada uma velocidade em torno de $850 \mathrm{~m} / \mathrm{s}$ para a correção NMO.

Figura 81 - Seção empilhada obtida a partir dos sismogramas de reflexão PS.

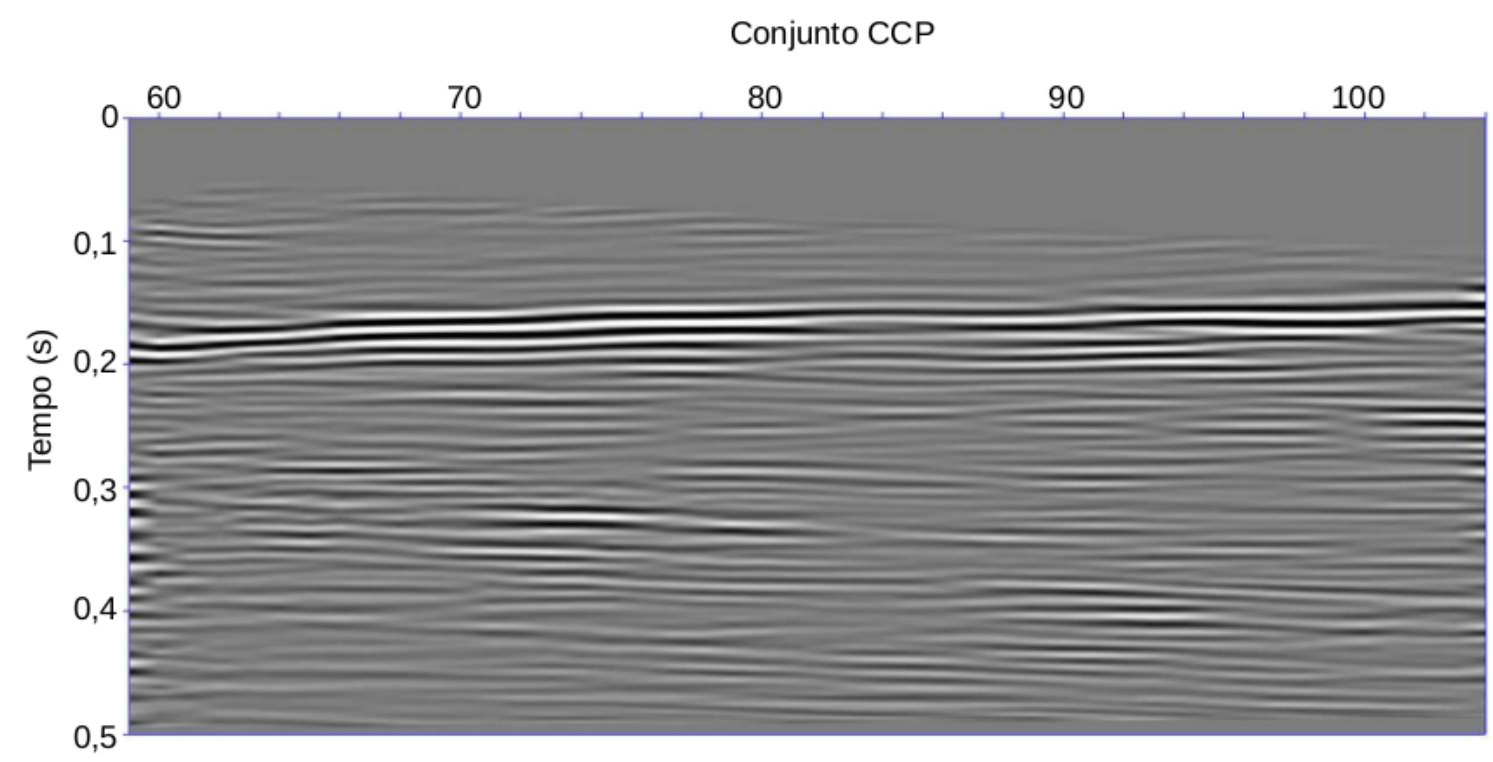


Figura 82 - Seção empilhada ( para a onda PS) em profundidade.

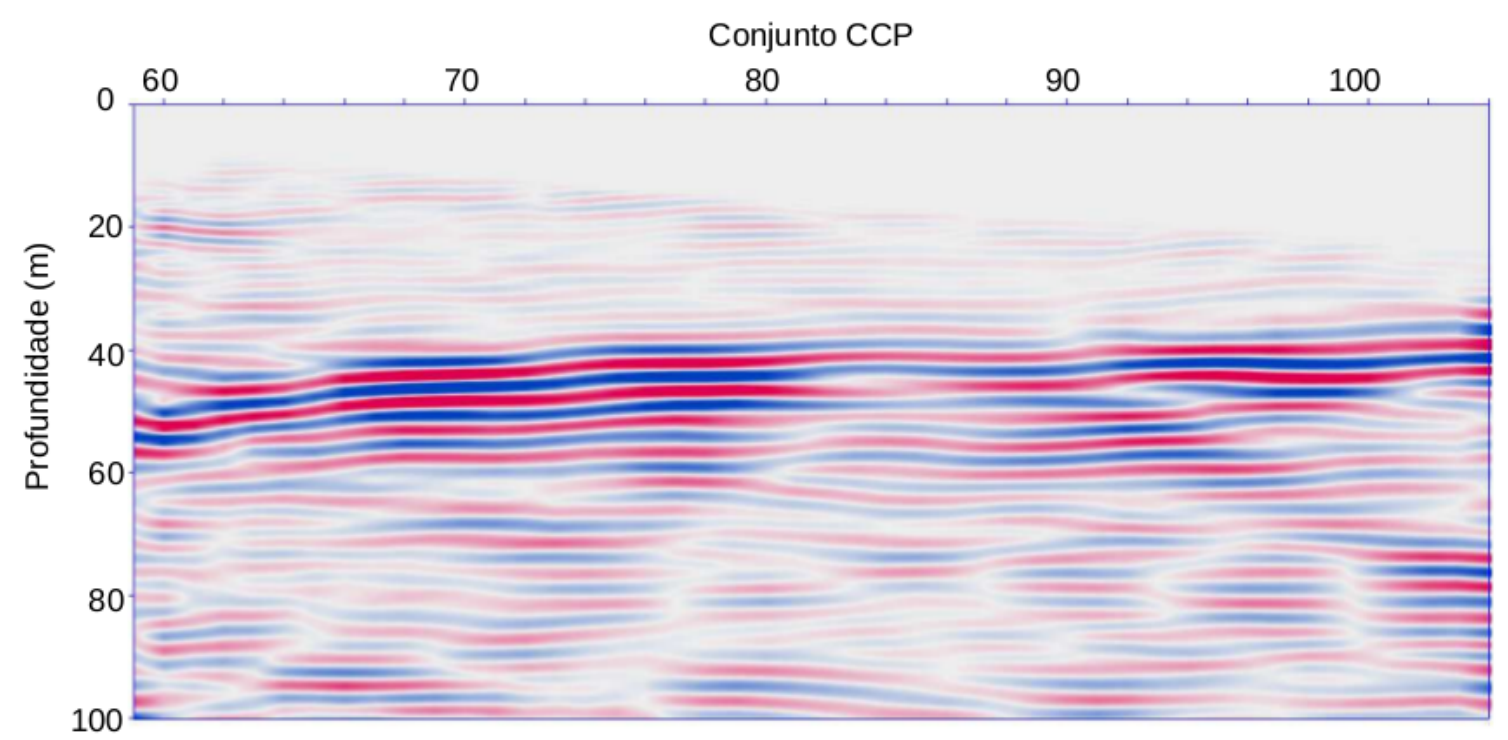

\subsubsection{Aplicação da FWI}

O foco desta aplicação foi a melhoria da velocidade da onda $\mathrm{S}$ acima do topo rochoso. A FWI foi aplicada sobre os conjuntos de tiros brutos com a adoção de um filtro passa-alta de $5 \mathrm{~Hz}$ e a correção 3D/2D.

Para evitar o problema de salto de ciclos a inversão iniciou com um filtro passabaixa de $10 \mathrm{~Hz}$ e foi aumentando de $10 \mathrm{~Hz}$ no decorrer das iterações. O aumento da frequência ocorre quando a diferença no valor da função objetivo é menor que 0,01 depois de duas iterações.

O pré-condicionamento do gradiente foi aplicado da mesma forma que para os dados sintéticos. Foi utilizado um taper de 3 metros de raio. Para a estimativa com a aproximada da Hessiana foi utilizada a aproximação baseada na teoria de Shin et al. (2001) com um peso de 0,5 a regularização da mesma (Equação 3.47).

Para o modelo inicial foram utilizadas as informações obtidas no processamento dos dados de refração, ondas superficiais e reflexão. Entretanto, foi feita a opção por modelos suaves com parâmetros crescendo com a profundidade. O modelo de densidades foi adotado com base em valores adequados para a geologia observada nos poços.

A wavelet utilizada como sinal emitido pela fonte foi uma wavelet de Ricker de $28 \mathrm{~Hz}$ baseada no tipo da fonte e na frequência dos geofones. A fonte utilizada gera uma wavelet de fase aproximadamente zero e os geofones de menor frequência possuem $28 \mathrm{~Hz}$.

A estimativa do valor do $\mathrm{Q}$ foi baseada no método de frequência de deslocamento centroide (QUAN; HARRIS, 1997), O método foi aplicado nos conjuntos de tiro com afastamento maior onde foi obtido o valor médio de 34, assim como mostrado no exemplo da Figura 84. 
Figura 83 - Modelos iniciais para a aplicação de FWI no dado real.
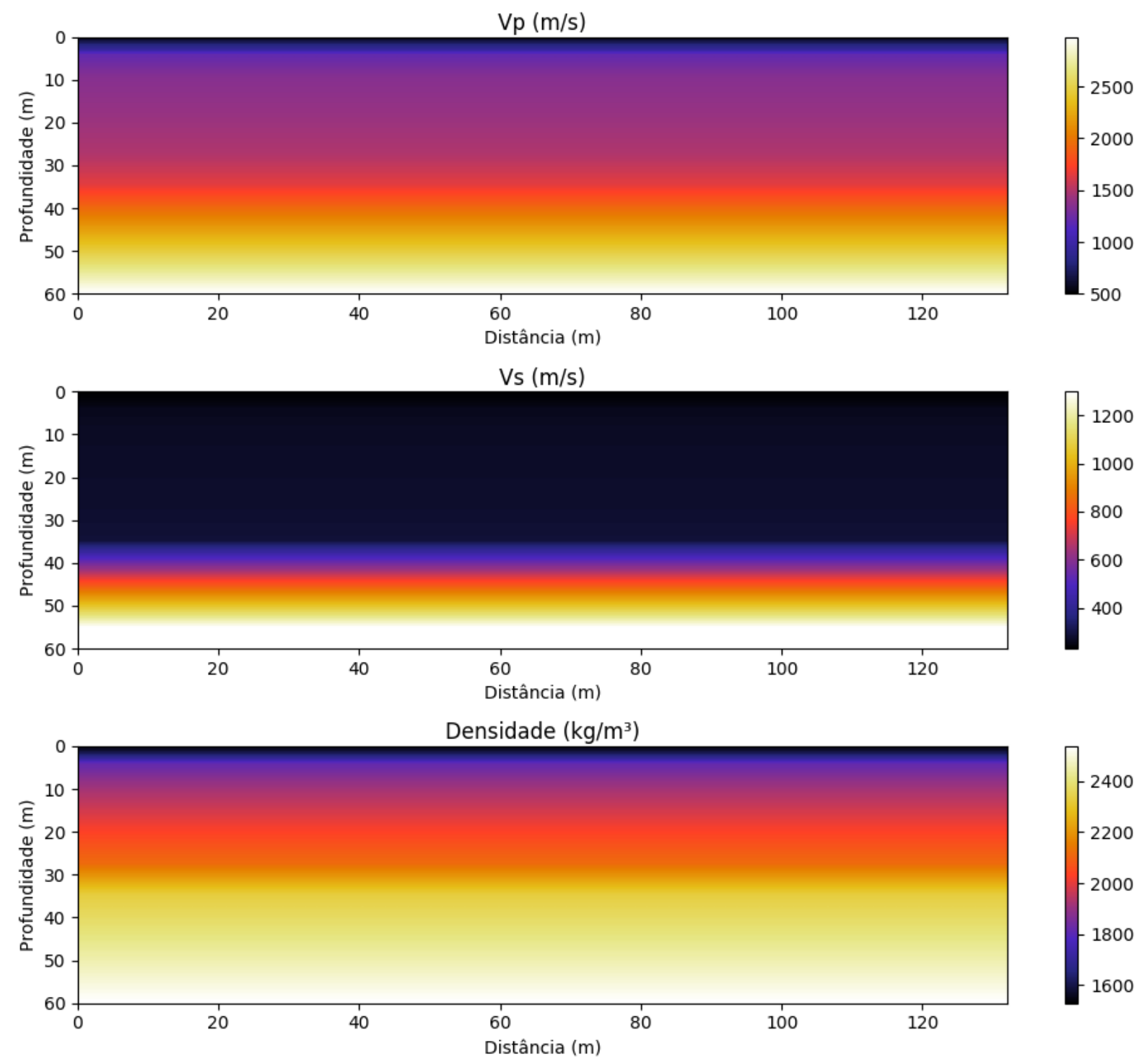

Com estas informações o método foi aplicado notando que as velocidades iniciais fornecidas para a velocidade da onda S não aumentam tanto com a profundidade quanto as obtidas no trabalho de Ullah, Prado e Lisa (2017). As baixas velocidades podem ser decorrentes de falhas na estimativa da velocidade da onda S através do processamento PS.

Os resultados mostrados nas Figuras 85 e 86 possuem valores mais altos para a velocidade da onda $\mathrm{S}$ acima do refletor. Os valores aumentaram em relação ao modelo inicial. Também foi percebida uma anomalia de baixa velocidade na parte central do modelo. As regiões de borda compreendem a camada PML onde a propagação da onda é atenuada para que não ocorram reflexões nas bordas do modelo. 
Figura 84 - Estimativa do valor do Q. (Cima) Espectro de amplitude para os traços 26 e 41 da componente vertical do conjunto de tiro 10; (Baixo) Valor estimado de Q.
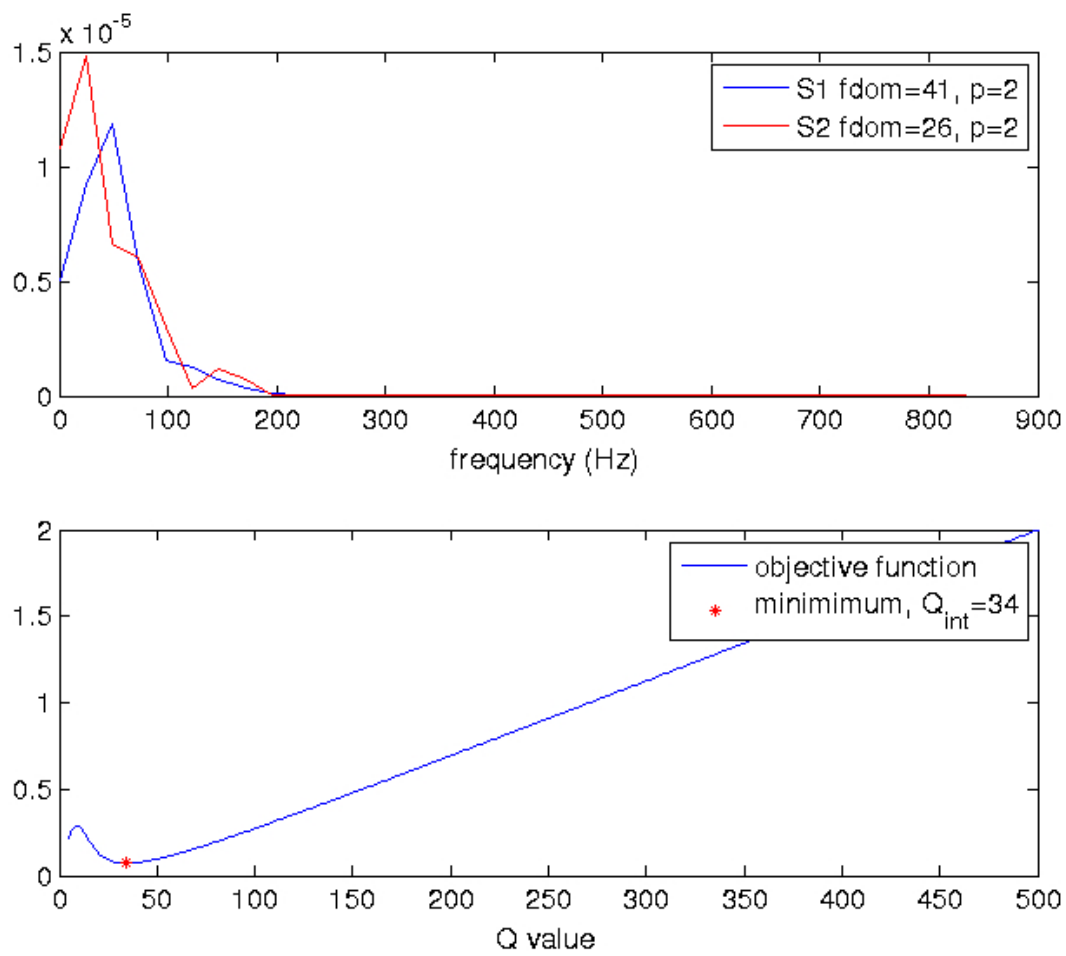

Figura 85 - Resultado final para a estimativa da velocidade da onda S.

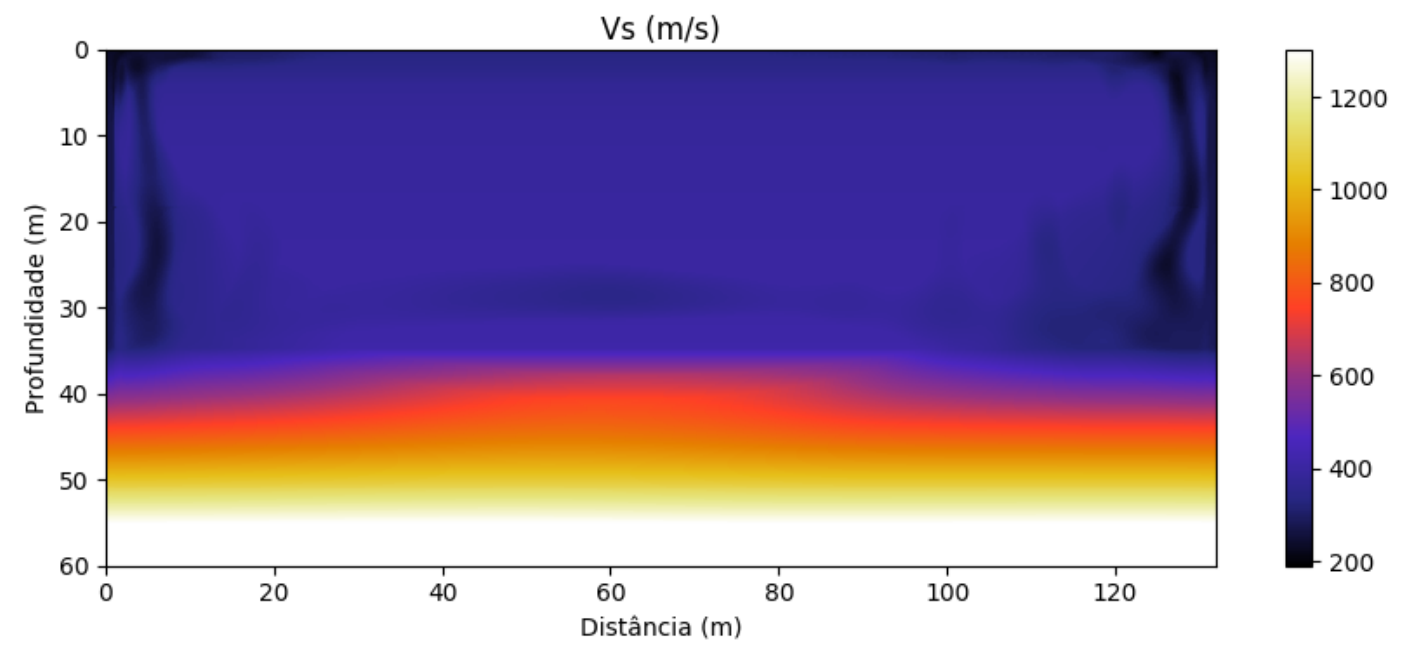


Figura 86 - Comparação entre o modelo inicial e o modelo final na parte central da Figura 85.

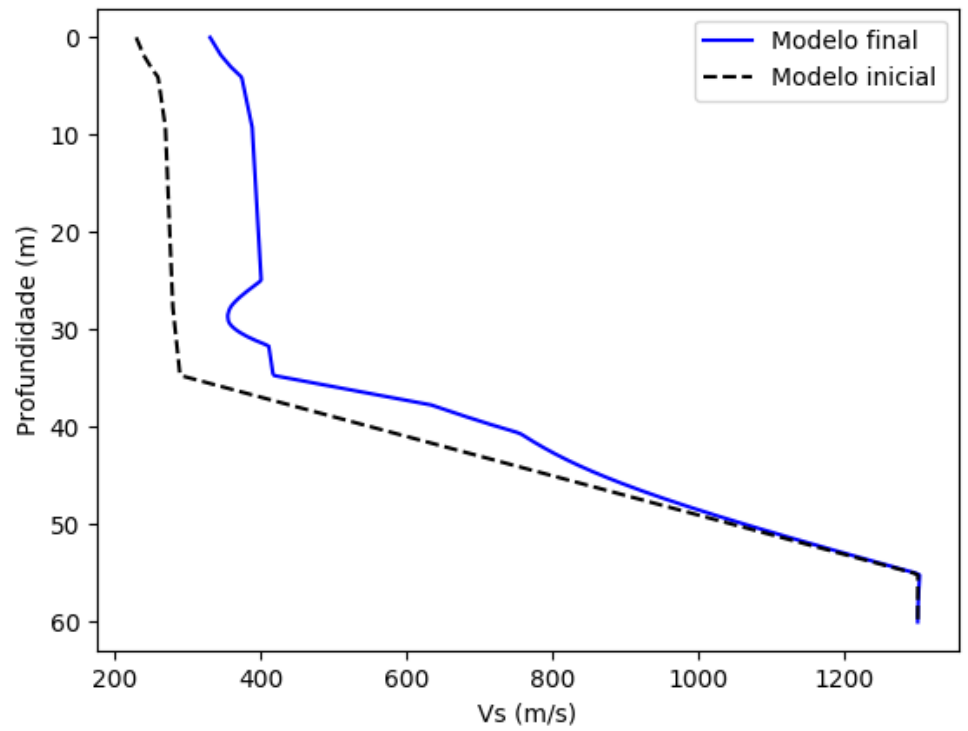




\section{Discussão e Conclusão}

Neste trabalho foi aplicado o método de inversão da forma de onda completa sobre dados de sísmica de reflexão rasa. A aplicação foi avaliada sobre dados sintéticos e reais. OCom os dados sintéticos foi validada a implementação do método e foram avaliados aspectos de sua execução voltados as particularidades dos dados sísmicos na escala de investigação rasa. Para validar o uso em dados reais optou-se por utilizar dados de um sítio controlado com informações geológicas de poço e de ensaio downhole disponíveis.

Uma das motivações deste trabalho foi a de aplicar a FWI em janelas de afastamentos longos nas componentes vertical e radial, de forma a utilizar os mesmos dados adquiridos para o uso da técnica CMP visando a reflexão do embasamento raso. O processamento de reflexões PP e PSv apresenta dificuldades para afastamentos longos em relação a profundidade dos refletores combinados à complexidade maior da reflexão PSv. Assim, uma das motivações principais do trabalho foi avaliar a capacidade do método em melhorar modelos de velocidades obtidos com o processamento PSv.

Nos dados sintéticos foi testada a estimativa dos parâmetros $V_{P}, V_{S}$ e $\rho$ tanto individualmente quanto concomitantemente. Também foi testada a aplicação do método de busca global Evolução Diferencial, como uma forma de avaliar a sensibilidade da estimativa de cada parâmetro em janelas de afastamentos diferentes. Este tipo de análise indicou uma sensibilidade maior para a velocidade da onda $\mathrm{S}$ em todos testes realizados. Uma sensibilidade maior para a velocidade da onda $\mathrm{S}$ na janela mais próxima da fonte era esperada, pois, os dados são dominados por ondas superficiais. É de conhecimento geral na literatura que a inversão de ondas superficiais é mais sensível ao parâmetro $V_{S}$. Em janelas mais afastadas da fonte a sensibilidade de todos os parâmetros diminui. Porém, o método continuou indicando boa sensibilidade para a determinação de $V_{S}$.

Os resultados para estimativa individual de parâmetros pelo método do gradiente conjugado em modelos 2D foram promissores para todos os parâmetros. Entretanto, foi notada a necessidade de um modelo $V_{P}$ muito próximo do real quando comparado aos demais parâmetros. A densidade não aparentou sofrer do mesmo problema nos testes realizados. Entretanto, os resultados obtidos para a densidade aparentam sofrer mais oscilações do que para os outros dois parâmetros.

A estimativa concomitante dos parâmetros com o gradiente conjugado apresentou bons resultados para a estimativa da velocidade da onda S. No caso da estimativa concomitante todos os modelos iniciais são diferentes do real. Logo, a estimativa se torna mais difícil para todos os parâmetros. Para o caso onde foi utilizada uma relação pré-definida, estabelecendo um vínculo entre os parâmetros também foram obtidos bons resultados para a estimativa da $V_{S}$. Neste caso, algum detrimento na resposta dos demais parâmetros 
pode ocorrer se a $V_{P}$ e a densidade não seguirem a mesma relação por todo o modelo. O benefício deste tipo de aplicação seria ajustar as posições de uma interface, como por exemplo no teste do modelo de camada inclinada.

Posteriormente a estes testes foram avaliados alguns parâmetros da inversão vinculados ao gradiente. Isto foi feito porque nos resultados $2 \mathrm{D}$ foi observado um efeito no resultado final relacionado a posição da fonte e dos geofones, causando um aumento do valor do parâmetro estimado próximo a estas regiões. A FWI sofre com uma menor sensibilidade em afastamentos maiores em relação a fonte e com altos valores dos gradientes próximos as posições das fontes. Uma das formas utilizadas no código para lidar com este problema é a aplicação de um filtro próximo a posição das fontes. Como se trata de uma aplicação rasa este filtro deve atingir apenas baixas profundidades. Outra forma de controlar o problema foi através da aplicação da aproximação Hessiana em conjunto com a soma de um valor na diagonal. Sendo assim, equivalente a uma regularização de Tikhonov de ordem zero. A aplicação da busca em conjunto com a Hessiana aproximada e devidamente regularizada se mostrou essencial para a obtenção de resultados próximos aos desejados. Outra forma de controlar estes efeitos é através de uma aquisição nos dois sentidos. Este tipo de aquisição favoreceria tanto o processamento PS quanto a aplicação da FWI. Como exemplo, nos testes "toy example"mostrados no manual do IFOS2D são obtidos bons resultados devido a iluminação simétrica do modelo.

A FWI também foi aplicada num modelo sintético de camada inclinada. Esta aplicação teve o intuito de buscar apenas a velocidade da onda S. Esta aplicação manteve os valores dos modelos $V_{P}$ e densidade em um modelo próximo ao modelo inicial $V_{S}$, ou seja, errados em relação ao modelo real. Os resultados permitiram observar uma região relacionada a inclinação da camada o que reforça a possibilidade da FWI de encontrar regiões anômalas.

Por fim, a aplicação em dados reais procurou avaliar uma possível melhoria da resposta obtida através do processamento PP e PS. O processamento dos eventos de reflexão PP e PS gerou boas seções empilhadas com um evento de reflexão coerente com a profundidade. Entretanto, a velocidade da onda S obtida com o processamento (Figura 83) se encontrava um pouco abaixo da esperada em relação aos resultados de Ullah, Prado e Lisa (2017). E os resultados obtidos com a aplicação da FWI aproximam a $V_{S}$ inicial para os valores desejados. Restando ainda uma zona anômala na parte central do modelo que deve ser verificada em trabalhos futuros.

Os resultados obtidos indicam que a aplicação conjunta da FWI com o processamento PP e PS são promissores. A FWI tem a capacidade de refinar os modelos de velocidade, mesmo sofrendo com uma menor sensibilidade em afastamentos maiores em relação a fonte e com altos valores dos gradientes próximos as posições das fontes.

Como recomendações futuras temos a aplicação da FWI como método para encontrar regiões anômalas, estruturas de engenharia por exemplo. Já existem algumas aplicações 
da FWI de ondas superficiais na tentativa de identificar sumidouros ou estruturas de concreto. Quanto à realização da FWI a adoção de outras formas de regularização também é um ponto importante neste sentido. A regularização adotada para controlar a Hessiana aproximada é do tipo mais simples e este problema tem sido pouco explorado em trabalhos de sísmica rasa. Regularizações que assumam a suavidade ou a compacidade de estruturas dos modelos podem ser de grande valia. 


\section{Referências}

ALEARDI, M.; MAZZOTTI, A. 1D elastic full-waveform inversion and uncertainty estimation by means of a hybrid genetic algorithm-Gibbs sampler approach.

Geophysical Prospecting, v. 65, n. 1, p. 64-85, jan 2017. ISSN 00168025. Disponível em: <http://doi.wiley.com/10.1111/1365-2478.12397>. Citado na página 43.

AMROUCHE, M.; YAMANAKA, H. Two-dimensional shallow soil profiling using timedomain waveform inversion. GEOPHYSICS, v. 80, n. 1, p. EN27-EN41, jan 2015. ISSN 0016-8033. Disponível em: <http://library.seg.org/doi/abs/10.1190/geo2014-0027.1>. Citado na página 14.

BALIS, E. Q-factor estimation through optimization approach to near-offset VSP data. In: SEG 2011 anuual meeting. [S.l.: s.n.], 2011. Citado na página 37.

BARBIER, M. The Mini-Sosie Method. Boston: Springer, 1983. 90 p. ISBN 978-0-934634-41-0. Citado 2 vezes nas páginas 79 e 91.

BATH, M. Developments in solid earth geophysics. [S.l.]: Elsevier Science Publishing Co., 1974. Citado na página 37.

BOHLEN, T. Parallel 3-D viscoelastic finite difference seismic modelling. Computers and Geosciences, v. 28, n. June 2001, p. 887-899, 2002. ISSN 00983004. Citado na página 21.

BOKHONOK, O. et al. Residual function dispersion maps to evaluate multidimensional objective function topography : Near-surface geophysical inverse problems. In: Fourteenth International Congress of the Brazilian Geophysical Society. Rio de Janeiro: [s.n.], 2015. p. 1-3. Citado na página 39.

BOKHONOK, O. E. D. P. C. d. S.; DIOGO, L. A. I.; PRADO, R. L. I. Velocity and density estimation from nonlinear amplitude inversion of prestack multicomponent shallow seismic data: A numerical model study. In: $13^{\circ}$ International Congress of the Brazilian Geophysical Society. [S.l.: s.n.], 2013. p. 1-6. Citado na página 39.

BRETAUDEAU, F. et al. 2D elastic full-waveform imaging of the near-surface: application to synthetic and physical modelling data sets. Near Surface Geophysics, v. 11, n. 1969, p. 1-10, jun 2013. ISSN 18730604. Disponível em: <http: //nsg.eage.org/publication/publicationdetails $/$ ?publication $=68284>$. Citado na página 14.

BUNKS, C. et al. Multiscale seismic waveform inversion. GEOPHYSICS, v. 60, n. 5, p. 1457-1473, sep 1995. ISSN 0016-8033. Disponível em: <http: //link.aip.org/link/?GPYSA7/60/1457/1http://library.seg.org/doi/10.1190/1.1443880>. Citado na página 34.

CARCIOnE, J. M.; HeRMAN, G. C.; KROODE, A. P. E. ten. Seismic modeling. GEOPHYSICS, v. 67, n. 4, p. 1304-1325, jul 2002. ISSN 0016-8033. Disponível em: <http://library.seg.org/doi/abs/10.1190/1.1500393>. Citado na página 16. 
CASTLE, R. A theory of normal moveout. Geophysics, v. 59, p. 983-999, 1994. Citado na página 86.

CHENG, P.; MARGRAVE, G. F. Estimation of Q : a comparison of different computational methods. v. 12, n. May, p. 2-5, 2013. Citado na página 37.

COHEN, J. K.; STOCKWELL, J. J. W. CWP/SU: Seismic Un*x Release No. 44: an open source software package for seismic research and processing. [S.l.]: Center for Wave Phenomena, Colorado School of Mines, 2015. Citado na página 97.

DIOGO, L. A.; Le Diagon, F. M. M.; PRADO, R. L. Bedrock imaging using post-critical shallow seismic reflection data. Journal of Applied Geophysics, v. 57, n. 1, p. 1-9, dec 2004. ISSN 09269851. Disponível em: < http://linkinghub.elsevier.com/retrieve/pii/ S092698510400059X>. Citado na página 93.

EMMERICH, H.; KORN, M. Incorporation of attenuation into time-domain computations of seismic wave fields. GEOPHYSICS, v. 52, n. 9, p. 1252-1264, sep 1987. ISSN 0016-8033. Disponível em: <http://library.seg.org/doi/10.1190/1.1442386>. Citado na página 21.

FENG, X.; REN, Q.; LIU, C. Quantitative imaging for civil engineering by joint full waveform inversion of surface-based GPR and shallow seismic reflection data. Construction and Building Materials, Elsevier Ltd, 2017. ISSN 09500618. Disponível em: < http://linkinghub.elsevier.com/retrieve/pii/S0950061817313685>. Citado na página 14.

FICHTNER, A. Full seismic waveform modelling and inversion. Full Seismic Waveform Modelling and Inversion by, 2011. Disponível em: $<$ http://www.geo.uu.nl/\{ $\}$ fichtner/ TableOfContents\{\_\}fichtne>. Citado na página 14.

FLIEDNER, M. M.; TREITEL, S.; MACGREGOR, L. Full-waveform inversion of seismic data with the Neighbourhood Algorithm. The Leading Edge, n. May, p. 570-579, 2012. Citado na página 39.

FORBRIGER, T.; GROOS, L.; SCHAFER, M. Line-source simulation for shallow-seismic data. Part 1: theoretical background. Geophysical Journal International, v. 198, n. 3, p. 1387-1404, 2014. ISSN 0956-540X. Disponível em: <http://gji.oxfordjournals.org/cgi/doi/10.1093/gji/ggu199>. Citado na página 34 .

GARDNER, G. H. F.; GARDNER, L. W.; GREGORY, A. R. FORMATION VELOCITY AND DENSITY - THE DIAGNOSTIC BASICS FOR STRATIGRAPHIC TRAPS. GEOPHYSICS, v. 39, n. 6, p. 770-780, dec 1974. ISSN 0016-8033. Disponível em: $<$ http://library.seg.org/doi/abs/10.1190/1.1440465>. Citado na página 27.

GEOMETRICS. SeisImager/2D Refraction Data Analysis Software. Disponível em: <http://www.geometrics.com/geometrics-products/seismographs/ download-seismograph-software $/\{\backslash \#\}$ SeisImager $>$. Citado na página 96.

GRECHKA, V.; TSVANKIN, I. PP + PS = SS. GEOPHYSICS, v. 67, n. 6, p. 1961-1971, nov 2002. ISSN 0016-8033. Disponível em: <http://library.seg.org/doi/abs/10.1190/1. $1527096>$. Citado 2 vezes nas páginas 85 e 86.

GROOS, L. 2D full waveform inversion of shallow seismic Rayleigh waves. 156 p. Tese (Doutorado) - Karlsruher Instituts fur Technologie, mar 2013. Citado 2 vezes nas páginas 34 e 66. 
GROOS, L. et al. Manual IFOS2D. 2017. 70 p. Citado 3 vezes nas páginas 16, 19 e 33.

GROOS, L. et al. Challenges for 2-D elastic Full waveform Inversion of Shallow-seismic Rayleigh Waves. In: 76th EAGE Conference 8 Exhibition 2014. [S.l.: s.n.], 2014. p. 3-5. Citado na página 14.

GROOS, L. et al. The role of attenuation in 2D full-waveform inversion of shallow-seismic body and Rayleigh waves. GEOPHYSICS, v. 79, n. 6, p. R247-R261, nov 2014. ISSN 0016-8033. Disponível em: <http://library.seg.org/doi/abs/10.1190/geo2013-0462.1>. Citado na página 34.

GROOS, L. et al. Application of a complete workflow for 2D elastic fullwaveform inversion to recorded shallow-seismic Rayleigh waves. GEOPHYSICS, v. 82, n. 2, p. R109-R117, mar 2017. ISSN 0016-8033. Disponível em: < http: //library.seg.org/doi/10.1190/geo2016-0284.1>. Citado 3 vezes nas páginas 16, 33 e 46.

HARDAGE, B. A. et al. Multicomponent Seismic Technology. Society of Exploration Geophysicists, 2011. 323 p. ISBN 978-1-56080-282-2. Disponível em: <http: //library.seg.org/doi/book/10.1190/1.9781560802891>. Citado 2 vezes nas páginas 83 e 84 .

HARRISON, M. Processing of P-SV surface-seismic data: Anisotropy analysis, dip moveout and migration. Tese (Thesis) - University of Calgary, 1992. Citado 2 vezes nas páginas 88 e 89.

HORN, J. Finite Markov Chain Analysis of Genetic Algorithms with Niching. Proceedings of the Fifth International Conference on Genetic Algorithms, p. 110-117, 1993. Disponível em: <citeseer.ist.psu.edu/article/horn93finite.html>. Citado na página 43.

IGEL, H. Computational Seismology: A Practical Introduction. Oxford University Press, 2016. 324 p. ISBN 9780191026850. Disponível em: < https://books.google.com.br/books? id=5254DQAAQBAJ>. Citado na página 21.

KARRAY, M.; LEFEBVRE, G. Significance and evaluation of Poisson's ratio in Rayleigh wave testing. Canadian Geotechnical Journal, v. 45, n. 5, p. 624-635, may 2008. ISSN 0008-3674. Disponível em: <http://dx.doi.org/10.1139/T08-016http: //www.nrcresearchpress.com/doi/abs/10.1139/T08-016>. Citado na página 26.

KÖHN, D. Time Domain 2D Elastic Full Waveform Tomography. 191 p. Tese (Doutorado) - Christian-Albrechts-Universität zu Kiel, 2011. Citado 2 vezes nas páginas 24 e 28.

KÖHN, D. et al. On the influence of model parametrization in elastic full waveform tomography. Geophysical Journal International, v. 191, n. 1, p. 325-345, 2012. ISSN 0956540X. Citado na página 33.

KÖHN, D. et al. 2D elastic full waveform tomography of synthetic marine reflection seismic data. Expanded Abstract: 72nd EAGE Conference 8 Exhibition, Euro. Asso. Geo. Eng., p. P385, 2010. Disponível em: <http://www.geophysik.uni-kiel.de/\{ $\}$ dkoehn/ publications/poster $\left\{\backslash \_\right.$EAGE $\left\{\backslash \_\right\}>$. Citado na página 16.

LEVANDER, A. R. Fourth-order finite-difference P-SV seismograms. Geophysics, v. 53, n. 11, p. 1425-1436, 1988. Citado 2 vezes nas páginas 16 e 19. 
MALOVICHKO, A. A. A new representation of the traveltime curve of reflected waves in horizontally layered media. Applied Geophysics (in Russian), v. 91, n. 1, p. 47-53, 1978. Citado na página 86.

MASHIMA, H. Irreplace friends. In: Fairy Tail. [S.l.]: Kodansha, 2017. cap. 545, p. 41. Citado na página 2.

MCVAY, M.; TRAN, K. T. Detection of Sinkholes or Anomalies Using Full Seismic Wave Fields. Florida, 2013. 1-65 p. Citado na página 14.

MENGXUAN, Z. et al. Time-domain full waveform inversion using the gradient preconditioning based on seismic wave energy: Application to the South China Sea. In: International Geophysical Conference, Qingdao, China, 17-20 April 201\%. [S.1.: s.n.], 2017. p. 440-443. Citado na página 66.

MOCZO, P. Introduction To Modeling Seismic Wave Propagation By the Finite-Difference Method. [S.l.: s.n.], 1998. 1-102 p. Citado na página 16.

MOCZO, P. et al. The finite-difference and finite-element modeling of seismic wave propagation and earthquake motion. Acta Physica Slovaca, v. 57, n. 2, p. 177-406, 2007. ISSN 03230465. Disponível em: <papers://2ee5b6b1-9ec6-4645-855c-1ac4fb8101af/Paper/ p5681>. Citado na página 21.

MOLDOVEANU-CONSTANTINESCU, C.; SACCHI, M. D. Enhanced resolution in Radon domain using the shifted hyperbola equation. In: SEG Technical Program Expanded Abstracts 2005. Society of Exploration Geophysicists, 2005. p. 2277-2280. Disponível em: $<$ http://library.seg.org/doi/abs/10.1190/1.2148171>. Citado na página 86.

MORA, P. Nonlinear two-dimensional elastic inversion of multioffset seismic data. Geophysics, v. 52, n. 9, p. 1211, 1987. ISSN 1070485X. Citado na página 13.

NUBER, A.; MANUKYAN, E.; MAURER, H. Enhancement of near-surface elastic full waveform inversion results in regions of low sensitivities. Journal of Applied Geophysics, Elsevier B.V., v. 122, p. 192-201, nov 2015. ISSN 09269851. Disponível em: $<$ http://linkinghub.elsevier.com/retrieve/pii/S0926985115300318>. Citado na página 33.

PLESSIX, R.-E.; MULDER, W. A. Frequency-domain finite-difference amplitudepreserving migration. Geophysical Journal International, v. 157, n. 3, p. 975-987, 2004. Disponível em: <http://dx.doi.org/10.1111/j.1365-246X.2004.02282.x>. Citado 2 vezes nas páginas 35 e 69.

PORSANI, J. L. et al. Summary for Policymakers. In: Intergovernmental Panel on Climate Change (Ed.). Climate Change 2013 - The Physical Science Basis. Cambridge: Cambridge University Press, 2004. v. 22, n. 3, p. 1-30. ISBN 9788578110796. Disponível em: < http://ebooks.cambridge.org/ref/id/CBO9781107415324A009>. Citado 2 vezes nas páginas 91 e 92 .

QUAN, Y.; HARRIS, J. M. Seismic attenuation tomography using the frequency shift method. Geophysics, v. 62, n. 3, p. 895, 1997. ISSN 1070485X. Citado 2 vezes nas páginas 37 e 103.

RICCOMINI, C. O rift continental do sudeste do Brasil. 319 p. Tese (Tese de doutorado) — Universidade de São Paulo, 1989. Citado na página 91. 
ROMDHANE, A. et al. Shallow-structure characterization by 2D elastic full-waveform inversion. Geophysics, v. 76, n. 3, p. R81-R93, 2011. ISSN 0016-8033. Disponível em: $<$ https://hal-brgm.archives-ouvertes.fr/hal-00593115>. Citado na página 14.

SCHÄFER, M. Application of full-waveform inversion to shallow-seismic Rayleigh waves on 2D structures. 159 p. Tese (Doutorado) - Karlsruhe, 2014. Disponível em: $<$ https://publikationen.bibliothek.kit.edu/1000041922>. Citado 2 vezes nas páginas 27 e 34 .

SCHAFER, M. et al. Line-source simulation for shallow-seismic data. Part 2: full-waveform inversion-a synthetic 2-D case study. Geophysical Journal International, v. 198, n. 3, p. 1405-1418, jul 2014. ISSN 0956-540X. Disponível em: $<$ http://gji.oxfordjournals.org/cgi/doi/10.1093/gji/ggu171https://academic.oup.com/ gji/article-lookup/doi/10.1093/gji/ggu171>. Citado na página 34.

SCHAFER, M. et al. 2D Full Waveform Inversion of Recorded Shallow Seismic Rayleigh Waves on a Significantly 2D Structure. In: Near Surface Geoscience 2013 - 19th European Meeting of Environmental and Engineering Geophysics. [S.1.: s.n.], 2013. p. 9-11. Citado na página 14.

SHIN, C. et al. Efficient calculation of a partial-derivative wave fi eld using reciprocity for seismic imaging and inversion. Geophysics, v. 66, n. 6, p. 1856-1863, 2001. Citado 2 vezes nas páginas 35 e 69.

SPADINI, A. et al. Analysis of Parameters Sensitivity in Seismic Full Waveform Inversion Applied to Shallow Subsurface. In: . [s.n.], 2015. p. 2013-2016. Disponível em: $<$ http://www.earthdoc.org/publication/publicationdetails $/$ ?publication $=82341>$. Citado na página 39.

SPADINI, A. S. et al. Strategies for the application of a conjugate gradient FWI algorithm of shallow environments. In: VII SimBGf. [S.l.: s.n.], 2016. Citado na página 55.

STORN, R.; PRICE, K. Differential Evolution - A simple and Efficient Heuristic for Global Optimization over Continuous Spaces. Journal of Global Optimization, v. 11, n. 4, p. 341-359, 1997. ISSN 09255001. Disponível em: <http://dx.doi.org/10.1023/A: 1008202821328http://link.springer.com/10.1023/A:1008202821328>. Citado 2 vezes nas páginas 24 e 36.

STOVAS, A.; URSIN, B. Estimation of layer parameters for linear P- and S-wave velocity functions. GEOPHYSICS, v. 72, n. 3, p. U27-U30, may 2007. ISSN 0016-8033. Disponível em: <http://library.seg.org/doi/abs/10.1190/1.2709423http: //library.seg.org/doi/10.1190/1.2709423>. Citado na página 86.

TANER, M. T.; KOEHLER, F. VELOCITY SPECTRA-DIGITAL COMPUTER DERIVATION APPLICATIONS OF VELOCITY FUNCTIONS. GEOPHYSICS, v. 34, n. 6, p. 859-881, dec 1969. ISSN 0016-8033. Disponível em: < http://library.seg.org/doi/ abs/10.1190/1.1440058http://library.seg.org/doi/10.1190/1.1440058>. Citado na página 85 .

TARANTOLA, A. Linearized inversion of reflection data. 1984. 998-1015 p. Citado na página 13. 
TARANTOLA, A. A strategy for nonlinear elastic inversion of seismic reflection data. Geophysics, v. 51, n. 10, p. 1893-1903, 1986. Citado na página 13.

TARANTOLA, A. Theoretical background for the inversion of seismic waveforms including elasticity and attenuation. Pure and Applied Geophysics PAGEOPH, v. 128, n. 1-2, p. 365-399, mar 1988. ISSN 0033-4553. Disponível em: <http: //link.springer.com/10.1007/BF01772605>. Citado na página 13.

TARANTOLA, A. Inverse Problem Theory. [S.l.]: SIAM, 2005. 358 p. ISBN 0-89871-572-5. Citado na página 28.

TESSMER, G.; BEHLE, A. COMMON REFLECTION POINT DATA-STACKING TECHNIQUE FOR CONVERTED WAVES1. Geophysical Prospecting, v. 36, n. 7, p. 671-688, oct 1988. ISSN 0016-8025. Disponível em: <http://doi.wiley.com/10.1111/j. 1365-2478.1988.tb02186.x>. Citado na página 85.

THOMSEN, L. Converted-wave reflection seismology over inhomogeneous, anisotropic media. GEOPHYSICS, v. 64, n. 3, p. 678-690, may 1999. ISSN 0016-8033. Disponível em: $<$ http://library.seg.org/doi/10.1190/1.1444577>. Citado 2 vezes nas páginas 79 e 83.

TRAN, K. T.; HILTUNEN, D. R. One-Dimensional Inversion of Full Waveforms using a Genetic Algorithm. Journal of Environmental \& Engineering Geophysics, v. 17, p. 197-213, 2012. Citado 2 vezes nas páginas 14 e 16.

TRAN, K. T.; HILTUNEN, D. R. Two-Dimensional Inversion of Full Waveforms Using Simulated Annealing. Journal of Geotechnical and Geoenvironmental Engineering, v. 138, n. 9, p. 1075-1090, sep 2012. ISSN 1090-0241. Disponível em: $<$ http://ascelibrary.org/doi/abs/10.1061/\{\\%\}28ASCE\{\\%\}29GT.1943-5606>. Citado 2 vezes nas páginas 14 e 16.

TRAN, K. T.; LUKE, B. Full waveform tomography to resolve desert alluvium. Soil Dynamics and Earthquake Engineering, Elsevier Ltd, v. 99, n. April, p. 1-8, 2017. ISSN 02677261. Disponível em: <http://linkinghub.elsevier.com/retrieve/pii/ S0267726116301087>. Citado na página 14.

TRAN, K. T.; MCVAY, M. Site characterization using Gauss-Newton inversion of 2-D full seismic waveform in the time domain. Soil Dynamics and Earthquake Engineering, Elsevier, v. 43, p. 16-24, dec 2012. ISSN 02677261. Disponível em: $<$ http://linkinghub.elsevier.com/retrieve/pii/S0267726112001613>. Citado na página 14.

TRAN, K. T. et al. Full Seismic Waveform Tomography at a Highly Variable Florida Site. p. 1-14, 2013. Citado na página 14.

TRAN, K. T. et al. Sinkhole detection using 2D full seismic waveform tomography. v. 78, n. 5, p. 175-183, 2013. ISSN 00168033. Citado na página 26.

TROMP, J.; TAPE, C.; LIU, Q. Seismic tomography, adjoint methods, time reversal and banana-doughnut kernels. Geophysical Journal International, v. 160, n. 1, p. 195-216, dec 2004. ISSN 0956540X. Disponível em: < https://academic.oup.com/gji/article-lookup/doi/ 10.1111/j.1365-246X.2004.02453.x>. Citado na página 27. 
ULLAH, I.; PRADO, R. L.; LISA, M. Single-station ellipticity retrieval and its joint inversion with dispersion curve, for a borehole test site. Arabian Journal of Geosciences, Arabian Journal of Geosciences, v. 10, n. 14, p. 316, jul 2017. ISSN 1866-7511. Disponível em: <http://link.springer.com/10.1007/s12517-017-3106-x>. Citado 6 vezes nas páginas 10, 91, 92, 93, 104 e 108.

VIRIEUX, J. P-SV wave propagation in heterogeneous media: Velocity-stress finite-difference method. Geophysics, v. 51, n. 4, p. 889-901, 1986. Citado na página 16.

VIRIEUX, J.; OPERTO, S. An overview of full-waveform inversion in exploration geophysics. GEOPHYSICS, v. 74, n. 6, p. WCC1-WCC26, nov 2009. ISSN 0016-8033. Disponível em: < http://library.seg.org/doi/abs/10.1190/1.3238367>. Citado na página 13.

WATANABE, M. D. Investigações sobre Análise de Velocidades e Empilhamento de Dados de Reflexão Sísmica Rasa. 72 p. Tese (Mestrado) — Universidade de São Paulo, 2010. Disponível em: <http://www.iag.usp.br/pos/sites/default/files/m\{\_\}michelle\{\_ \}watana $>$. Citado na página 86.

WATHELET, M. An improved neighborhood algorithm: Parameter conditions and dynamic scaling. Geophysical Research Letters, v. 35, n. 9, p. L09301, may 2008. ISSN 0094-8276. Disponível em: <http://doi.wiley.com/10.1029/2008GL033256>. Citado na página 96.

YILMAZ, Ö. Seismic Data Analysis. Society of Exploration Geophysicists, 2001. ISBN 978-1-56080-094-1. Disponível em: < http://library.seg.org/doi/book/10.1190/1. 9781560801580>. Citado na página 90.

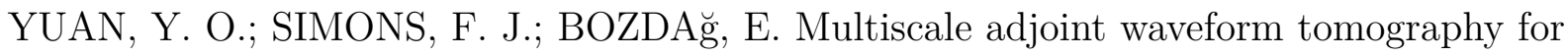
surface and body waves. Geophysics, v. 80, n. 5, p. R281-R302, 2015. ISSN 0016-8033. Disponível em: <http://library.seg.org/doi/10.1190/geo2014-0461.1>. Citado na página 14. 